\title{
A comprehensive overview of radioguided surgery using gamma detection probe technology
}

\author{
Stephen P Povoski*1, Ryan L Neff1', Cathy M Mojzisik1,2, David M O'Malley3, \\ George H Hinkle ${ }^{2,4}$, Nathan C Hall2 ${ }^{2}$ Douglas A Murrey Jr${ }^{2}$, \\ Michael V Knopp ${ }^{2}$ and Edward W Martin Jr${ }^{1}$
}

\begin{abstract}
Address: ${ }^{1}$ Division of Surgical Oncology, Department of Surgery, Arthur G. James Cancer Hospital and Richard J. Solove Research Institute and Comprehensive Cancer Center, The Ohio State University, Columbus, OH, 43210, USA, 2Department of Radiology, The Ohio State University, Columbus, OH, 43210, USA, ${ }^{3}$ Division of Gynecologic Oncology, Department of Obstetrics and Gynecology, Arthur G. James Cancer Hospital and Richard J. Solove Research Institute and Comprehensive Cancer Center, The Ohio State University, Columbus, OH, 43210, USA and ${ }^{4}$ College of Pharmacy, The Ohio State University, Columbus, OH, 43210, USA

Email: Stephen P Povoski* - stephen.povoski@osumc.edu; Ryan L Neff - ryan.neff@mercy.net; Cathy M Mojzisik - cathy.mojzisik@osumc.edu; David M O'Malley - david.omalley@osumc.edu; George H Hinkle - hinkle.5@osu.edu; Nathan C Hall - nathan.hall@osumc.edu;

Douglas A Murrey - douglas.murrey@osumc.edu; Michael V Knopp - knopp.16@osu.edu; Edward W Martin - edward.martin@osumc.edu

* Corresponding author
\end{abstract}

Published: 27 January 2009

World Journal of Surgical Oncology 2009, 7:1I doi:10.1186/1477-7819-7-11
Received: 21 December 2008

Accepted: 27 January 2009

This article is available from: http://www.wjso.com/content/7///II

(C) 2009 Povoski et al; licensee BioMed Central Ltd.

This is an Open Access article distributed under the terms of the Creative Commons Attribution License (http://creativecommons.org/licenses/by/2.0), which permits unrestricted use, distribution, and reproduction in any medium, provided the original work is properly cited.

\begin{abstract}
The concept of radioguided surgery, which was first developed some 60 years ago, involves the use of a radiation detection probe system for the intraoperative detection of radionuclides. The use of gamma detection probe technology in radioguided surgery has tremendously expanded and has evolved into what is now considered an established discipline within the practice of surgery, revolutionizing the surgical management of many malignancies, including breast cancer, melanoma, and colorectal cancer, as well as the surgical management of parathyroid disease. The impact of radioguided surgery on the surgical management of cancer patients includes providing vital and realtime information to the surgeon regarding the location and extent of disease, as well as regarding the assessment of surgical resection margins. Additionally, it has allowed the surgeon to minimize the surgical invasiveness of many diagnostic and therapeutic procedures, while still maintaining maximum benefit to the cancer patient. In the current review, we have attempted to comprehensively evaluate the history, technical aspects, and clinical applications of radioguided surgery using gamma detection probe technology.
\end{abstract}

\section{Background}

The concept of radioguided surgery using a radiation detection probe system originated approximately 60 years ago. Interestingly, the first recognized description of radioguided surgery involving a radiation detection probe system [1] did not involve a gamma detection probe, but instead involved the use of a gaseous ionization detector called a Geiger-Müller tube [2], which has a high sensitiv- ity for beta radiation emitting radionuclides and a very low sensitivity for gamma radiation emitting radionuclides.

In 1949, Selverstone et al [1] at Harvard Medical School reported on 33 suspected brain tumor patients that were intravenously injected with the beta radiation emitter, phosphorus-32 (32P). At surgery, using a handheld Gei- 
ger-Müller tube device, counts in the area of suspected tumor and normal brain tissue were obtained at various time intervals and various depths beneath the cerebral cortex. Following successful location of the tumor, attempts were made to demarcate its tumor boundary margins using the Geiger-Müller counter. Of 33 evaluated patients, 23 brain tumors ( $88 \%$ ) were localized using the Geiger-Müller counter. In 12 patients, the Geiger-Müller counter was used to facilitate total extirpation of tumor. In four patients, tumor was not localized by means of the Geiger-Müller counter. This included two false-negative results that were attributed to the inability to place the Geiger-Müller counter in close proximity to the tumor, one patient with diffuse infiltration of the entire cerebral hemisphere with tumor that precluded distinguishing it from normal adjacent tissue, and one patient in which no tumor was correctly identified.

It was then not until 1956, when Harris et al [3] at the Oak Ridge Institute of Nuclear Studies Medical Hospital reported the first description of radioguided surgery involving a gamma detection probe system. In their published report, a patient with a history of thyroid cancer who had previously undergone a total thyroidectomy some three years earlier and who had persistent iodine uptake in the neck region was intravenously injected with the gamma radiation emitter, iodine-131 (131I). At surgery, using a handheld scintillation detector device as the gamma detection probe, they localized and successfully resected an area of residual thyroid tissue.

Since the time of these landmark reports by Selverstone et al [1] and Harris et al [3], the concept of radioguided surgery and its supporting technologies has tremendously expanded and has evolved into what is now considered an established discipline within the practice of surgery, revolutionizing the surgical management of many malignancies. Along the way, various milestones in radioguided surgery have been reached (Table 1), and the clinical application of this technology has, to varying degrees, impacted upon almost every facet of cancer-related surgery (Table 2). The impact of radioguided surgery on the surgical management of cancer patients includes providing vital and real-time information to the surgeon regarding the location and extent of disease, as well as regarding the assessment of surgical resection margins. Additionally, it has allowed the surgeon to minimize the surgical invasiveness of many diagnostic and therapeutic procedures, while still maintaining maximum benefit to the cancer patient.

\section{Gamma detection probe systems}

Numerous handheld intraoperative radiation detection probe systems have been developed and have been made commercially available for use in radioguided surgery [423]. Such intraoperative radiation detection probes are

Table I: Historical timeline for milestones in radioguided surgery

\begin{tabular}{|c|c|}
\hline Year & Milestone \\
\hline 1949 & $\begin{array}{l}\text { Selverstone et al [I] at Harvard Medical School (Boston, Massachusetts, USA) were the very first to report the concept of radioguided } \\
\text { surgery using of a Geiger-Müller tube device and } 32 \mathrm{P} \text { to detect brain tumors. }\end{array}$ \\
\hline 1956 & $\begin{array}{l}\text { Harris et al [3] at the Oak Ridge Institute of Nuclear Studies Medical Hospital (Oak Ridge, Tennessee, USA) were the first to report the } \\
\text { application of a gamma detection probe during radioguided surgery using }|3| \mid \text { to detect residual thyroid tissue. }\end{array}$ \\
\hline 1981 & $\begin{array}{l}\text { Harvey et al [743] at Presbyterian Hospital of Dallas (Dallas, Texas, USA) first reported the application of a gamma detection probe for } \\
\text { radioguided biopsy of benign and metastatic bone lesions using } 99 \mathrm{mTc} \text { methylene diphosphonate. }\end{array}$ \\
\hline 1981 & $\begin{array}{l}\text { Ghelman et al [728] at The Hospital for Special Surgery (New York, New York, USA) first reported the application of a gamma detection } \\
\text { probe for radioguided resection of a benign bone lesion using } 99 \mathrm{mTc} \text { methylene diphosphonate. }\end{array}$ \\
\hline 1984 & $\begin{array}{l}\text { Aitken et al }[289,290] \text { at The Ohio State University (Columbus, Ohio, USA) first reported radioimmunoguided surgery using } 131 \mid- \text { labeled } \\
\text { anti-CEA polyclonal antibody to detect colorectal cancer. }\end{array}$ \\
\hline 1984 & $\begin{array}{l}\text { Ubhi et al. [408] at Queen's Medical Center/University Hospital (Nottingham, England, UK) first reported radioguided surgery using 20ITI- } \\
\text { thallous chloride for the detecting a parathyroid adenoma. }\end{array}$ \\
\hline 1987 & $\begin{array}{l}\text { Sickle-Santanello et al [299] at The Ohio State University (Columbus, Ohio, USA) first reported radioimmunoguided surgery using }{ }^{125} \text { I- } \\
\text { labeled anti-TAG-72 monoclonal antibody to detect colorectal cancer. }\end{array}$ \\
\hline 1993 & $\begin{array}{l}\text { Krag et al [135] at The University of Vermont (Burlington, Vermont, USA) first reported radioguided sentinel lymph node biopsy using } \\
99 \mathrm{mTc} \text { radiocolloid for breast cancer. }\end{array}$ \\
\hline 1993 & $\begin{array}{l}\text { Alex et al [188] at The University of Vermont (Burlington, Vermont, USA) first reported radioguided sentinel lymph node biopsy using } \\
99 \mathrm{mTc} \text { radiocolloid for malignant melanoma. }\end{array}$ \\
\hline 1995 & $\begin{array}{l}\text { Martinez et al [409] at The Ohio State University (Columbus, Ohio, USA) first reported use of } 99 \mathrm{mTc}-\mathrm{MIBI} \text { for the detecting parathyroid } \\
\text { gland pathology. }\end{array}$ \\
\hline 1997 & $\begin{array}{l}\text { Norman and Chheda [4I0] at The University of South Florida (Tampa, Florida, USA) popularized the technique of minimally-invasive } \\
\text { radioguided surgery using } 99 \mathrm{~m} \text { Tc-MIBI for the surgical management of primary hyperparathyroidism. }\end{array}$ \\
\hline 1999 & $\begin{array}{l}\text { Desai et al }[35,36] \text { at The Ohio State University (Columbus, Ohio, USA) first reported use of } 18 \text { F-FDG-directed surgery in the surgical } \\
\text { management of colorectal cancer. }\end{array}$ \\
\hline 2008 & $\begin{array}{l}\text { Strong et al [29] at Memorial Sloan-Kettering Cancer Center (New York, New York, USA) first reported radioimmunoguided surgery } \\
\text { using }|24| \text {-labeled monoclonal antibody specific for clear cell renal cell cancer. }\end{array}$ \\
\hline
\end{tabular}


Table 2: Clinical applications of radioguided surgery using gamma detection probe technology

\begin{tabular}{|c|c|}
\hline Clinical applications & Specific type(s) of radioguided surgery applications \\
\hline Breast cancer & RGSLNB, RIGS, ROLL, RIME, FDGDS \\
\hline \multicolumn{2}{|l|}{ Cutaneous malignancies } \\
\hline Malignant melanoma & RGSLNB, FDGDS \\
\hline Merkel cell carcinoma & RGSLNB \\
\hline Other cutaneous malignancies & RGSLNB \\
\hline \multicolumn{2}{|l|}{ Gastrointestinal malignancies } \\
\hline Colorectal cancer & RIGS, RGSLNB, FDGDS \\
\hline Anal cancer & RGSLNB \\
\hline Esophageal cancer & RGSLNB \\
\hline Gastric cancer & RGSLNB, RIGS, FDGDS \\
\hline Pancreatic cancer & RIGS \\
\hline GIST & FDGDS \\
\hline \multicolumn{2}{|l|}{ Head and neck malignancies } \\
\hline Squamous cell cancer & RGSNLB, RIGS, FDGDS \\
\hline Parathyroid disease & RGS \\
\hline Thyroid cancer & RGS, FDGDS, RGSLNB \\
\hline Parotid gland cancer & RGSLNB \\
\hline \multicolumn{2}{|l|}{ Gynecologic malignancies } \\
\hline Vulvar cancer & RGSLNB \\
\hline Vaginal carcinoma & RGSLNB \\
\hline Cervical cancer & RGSLNB \\
\hline Endometrial cancer & RGSLNB \\
\hline Ovarian Cancer & RIGS, FDGDS \\
\hline \multicolumn{2}{|l|}{ Urologic malignancies } \\
\hline Penile cancer & RGSLNB \\
\hline Prostate cancer & RGSLNB, RIGS \\
\hline Testicular cancer & RGSLNB, FDGDS \\
\hline Bladder cancer & RGSLNB \\
\hline Renal cell cancer & RIGS \\
\hline \multicolumn{2}{|l|}{ Thoracic malignancies } \\
\hline Lung cancer & RGSLNB, RIGS, RGS, FDGDS \\
\hline Pulmonary nodules & RGS \\
\hline \multicolumn{2}{|l|}{ Neuroendocrine tumors } \\
\hline GEP neuroendocrine tumors & RGS \\
\hline Bronchial carcinoids & RGS \\
\hline Neuroblastoma & RGS \\
\hline Pheochromocytoma & RGS \\
\hline Adrenocortical carcinoma & FDGDS \\
\hline Sarcoma & RGSLNB \\
\hline Brain tumors & RGS \\
\hline Bone lesions & RGS \\
\hline Lymphoma & RGS \\
\hline Monitoring of isolated limb perfusion & RGS \\
\hline
\end{tabular}

RGS, radioguided surgery; RIGS, radioimmunoguided surgery; RGSLNB, radioguided sentinel lymph node biopsy; ROLL, radioguided occult lesion localization; RIME, radioguided intraoperative margins evaluation; FDGDS, ${ }^{8}$ F-FDG directed surgery; GIST, gastrointestinal stromal tumor; GEP, gastroenteropancreatic

divided into two general categories (i.e., gamma detection probes and beta detection probes), based upon the specific type of radiation detected. Gamma probes detect photon radiation, consisting of either gamma rays or $\mathrm{x}$ rays $[10,11,17]$. Beta probes detect beta radiation, consisting of either positrons (positively charged electrons) or negatrons (negatively charged electrons) [10,11,18-23]. This includes some beta detection probe systems that are reported to have gamma photon background rejection capabilities $[22,23]$. However, the present review will spe- cifically concentrate upon the use of gamma detection probe technology in radioguided surgery. Additionally, the present review will not specifically discuss or advocate the use of any individual commercially-available brand names of gamma detection probe technology.

\section{Important performance variables of gamma detection probe systems}

The most important performance variables of any given gamma detection probe system consist of: (1) overall sen- 
sitivity (efficiency); (2) spatial selectivity (radial sensitivity distribution); (3) spatial resolution (lateral sensitivity distribution); (4) energy resolution (spectral discrimination); and (5) contrast $[9,11,13-15,17]$. Overall sensitivity (efficiency) is the detected count rate (photons detected) per unit of activity (photons emitted) and is determined at the tip of the probe profile. Spatial selectivity (radial sensitivity distribution) is described by the width of the resultant measurement cone out of which radiation is being detected at a defined distance. With a wider measurement cone, background signal may exceed target source signal and can lead to interference with detection of the target signal. With a narrower measurement cone, background counts will be reduced and detection of the target source signal will be more likely, even in the presence of an increased background signal or noise. Spatial resolution (lateral sensitivity distribution) is the ability of the gamma detection probe to accurately localize the position of a target source of activity, as well as to separate and distinguish two target sources of activity which are located relatively close to each other. Energy resolution (spectral discrimination) is the capacity of the gamma detection system to discriminate between emitted radiation of differing energies. Such energy discrimination is critical in two particular respects. First, it is critical for distinguishing two simultaneously administered radionuclides that have differing energies. Second, it is critical for distinguishing primary photons from scattered photons when higherenergy radionuclides are administered. Finally, contrast, which is directly related to all of the above performance variables of the gamma detection probe system, reflects the ability of the gamma detection probe to distinguish activity within the target tissue from that of the lower background activity within the surrounding non-target tissue.

\section{Basic principles of the radiation detector source housed within the gamma detection probe system}

Two general categories of gamma detection probe systems exist that can be utilized within the operating room environment. These include gamma detection probe systems that utilize a scintillation detector and gamma detection probe systems that utilize a semiconductor ionization detector $[4,6,8,10-12,15-17]$. Only crystalline materials are used as the detector source within commercially-available gamma detection probes.

Crystalline materials that have been utilized in scintillation detectors include thallium-activated sodium iodide (NaI $[\mathrm{Tl}]$ ), thallium-activated cesium iodide ( $\mathrm{CsI}[\mathrm{Tl}]$ ), samarium-activated lutetium ortho-oxysilicate (LSO), and bismuth germanate (BGO). The basic principle behind how a scintillation-type detection system works is that the radiation emitted from the radionuclide excites atoms within the scintillation crystal and produces visible light in proportion to the energy absorbed. A photomultiplier tube is used to enhance the resultant visible light that is produced and is then converted into an electrical pulse that is collected by the detection unit. Crystalline materials that have been utilized in semiconductor ionization detectors include cadmium telluride (CdTe), cadmium zinc telluride (CdZnTe), and mercuric iodide $\left(\mathrm{HgI}_{2}\right)$. The basic principle behind how a semiconductor ionizationtype detection system works is that the radiation emitted from the radionuclide produces free electrons as it passes through and ionizes the semiconductor crystal. The resultant free electrons that are produced then create an electrical pulse that is collected and amplified by the detection unit.

There are advantageous and disadvantageous features that are specific to scintillation-type detection systems and to semiconductor ionization-type detection systems $[4,6,8,10-12,15,17]$. On one hand, scintillation-type detection systems have higher sensitivity (especially for medium-energy to high-energy gamma photons), but have poorer energy resolution and scatter rejection. Likewise, scintillation-type detection probes tend to have a much bulkier probe head profile design. On the other hand, semiconductor ionization-type detection systems have higher energy resolution and scatter rejection, but have lower sensitivity (especially for medium-energy to high-energy gamma photons). Likewise, semiconductor ionization-type detection probes tend to have a much more compact probe head profile design.

Factors important in the appropriate selection of a gamma detection probe system for its intended clinical application Several factors are important in the appropriate selection of a particular gamma detection probe system $[4,5,8,9,11,15,17]$.

First, the specific radionuclide utilized and its particular gamma photon energy level is very important in the appropriate selection of a particular gamma detection probe system $[8,11,17]$. Whereas technetium-99m ( ${ }^{99 \mathrm{~m} T c)}$ labeled agents have been used almost exclusively for radioguided sentinel lymph node biopsy (SNL) procedures, various other radiopharmaceutical agents, such as monoclonal antibodies bound to various radionuclides (most commonly iodine radionuclides, indium-111


a nonphysiologic analog of glucose have also been used in radioguided surgical resection of tumors. While most commercially available gamma detection probe systems have relatively high sensitivity (efficiency) for predominantly lower energy gamma photon emitting radionuclides (such as iodine-125 (125I) and ${ }^{99 \mathrm{~m}} \mathrm{Tc}$ ), this may not necessarily be the case for predominantly higher energy gamma photon emitting radionuclides (such as ${ }^{131} \mathrm{I}$ ) or 
positron emitting radionuclides that produce high-energy gamma photons from resultant positron-electron annihilation (such as iodine-124 $\left({ }^{124} \mathrm{I}\right)$ and $\left.{ }^{18} \mathrm{~F}\right)$. As such, these resultant high-energy gamma photons remain an ongoing challenge for the gamma detection probe systems that are currently commercially available and has been the focus of recent product development of gamma detection probe systems that are specifically intended for the detection of high-energy gamma photons.

Second, the nature of the surgical procedure to be performed is important in the appropriate selection of a particular gamma detection probe system $[8,9,11,15,17]$. On one hand, gamma detection probe systems used for radioguided sentinel lymph node procedures require exceptional spatial resolution in order to allow for more precise localization of small lymph node candidates. On the other hand, gamma detection probe systems used for radioguided surgical resection of tumors requires high sensitivity in order to help guide the surgeon to the specific sites of disease while rapidly searching over a relatively large surgical field.

Third, the necessity for shielding and collimation of the head of the probe housing the crystalline material is also critical in the appropriate selection of a particular gamma detection probe system $[4,5,8,9,11,15,17]$. These features may already be built into the standard probe head or can be added onto the existing standard probe head. The function of shielding (material such as lead, tungsten, gold, or platinum) and collimation (length and aperture of the collimator) is to prevent attenuated radiation from unintended locations (i.e., scatter) from accessing the detector source within the probe head and thus producing unintended counts that are recognized by the gamma detection system. Side and back shielding of the probe head can be rather important when there is a strong and localized radiation source (i.e., the ${ }^{99 \mathrm{mTc}} \mathrm{T}$-labeled agent injection site for a radioguided SLN procedure) which lies in close proximity to the intended target (i.e., the SLN) or when utilizing higher energy gamma photon emitting radionuclides (such as ${ }^{131} \mathrm{I}$ ) or positron emitting radionuclides that produce high-energy gamma photons from resultant positron-electron annihilation (such as ${ }^{124}$ I and ${ }^{18} \mathrm{~F}$ ). It is clear that collimation of the gamma detection probe head results in improved spatial resolution and contrast between the emitted radiation from the intended target as compared to emitted radiation from surrounding non-target tissue (especially in areas of higher background activity). However, at the same time, such collimation produces a resultant loss in the sensitivity of the gamma detection probe system by decreasing the effectual detection aperture and lengthening the distance to the actual detection source. Thicker shielding and/or longer collimation is generally necessary when using higher energy gamma photon producing radionuclides. However, the addition of thicker shielding and/or longer collimation will increase the overall weight and size-dimensions of the gamma detection probe.

\section{Desirable design features of any given gamma detection probe system that are important to the surgeon}

Many design features of any given gamma detection probe system may be important to the surgeon $[8,9,11,12,15,17]$. The presence or absence of such specific design features may make any particular gamma detection probe system more or less attractive to the surgeon. First and foremost, the weight, shape, and ergonomic design of the gamma detection probe are critical. By far, surgeons favor sleekly designed, pencil-thin, lightweight probes and angulation of the detector head for better access to desired detection locations. While pencil-thin probes may offer higher spatial resolution secondary to their smaller detector size, they, unfortunately, yield a lower sensitivity than do larger-sized detector probes and can limit the degree of attainable shielding and collimation. Second, the audible signal and digital display of the gamma detection control unit are important variables for providing critical output information to the surgeon as to the localization of the radionuclide to the area of interest without distracting the surgeon from the overall activities within the surgical field [8]. Third, flexibility and adaptability of any given system with regards to removable side shielding, interchangeable collimators, interchangeable detection probes, and user-adjustable energy windows for different radionuclides is also critical to the overall design of a given gamma detection probe system. Lastly, the recent development of handheld, self-contained gamma detection probe systems [24], as well as wireless gamma detection probe technology that is adaptable to existing gamma detection probe systems [25] may help to further advance the technology involved in radioguided surgery by eliminating the need for cables within the surgical field that previously connected the gamma detection probe itself to the gamma detection control unit [25]. All these technology developments involving gamma detection probe systems may ultimately provide the surgeon with more flexibility for utilization of these innovative devices within the operating room environment.

\section{Properties of radionuclides utilized in radioguided surgery}

Numerous radionuclides have been utilized with the gamma detection probe in radioguided surgery. This includes, in alphabetical order, cobalt-57 $\left({ }^{57} \mathrm{Co}\right),{ }^{18} \mathrm{~F}$, gallium-67 $\left({ }^{67} \mathrm{Ga}\right),{ }^{111} \mathrm{In}$, iodine-123 (123I), ${ }^{124} \mathrm{I},{ }^{125} \mathrm{I},{ }^{131} \mathrm{I}$, $99 \mathrm{mTc}$, and thallium-201 $\left.{ }^{201} \mathrm{Tl}\right)$ [26]. The physical halflife, principle gamma photon radiation emission(s), and emission probability per decay (photon yield) of each of these radionuclides are summarized in Table 3[27]. In 
Table 3: Physical properties of radionuclides that have been utilized with the gamma detection probe in radioguided surgery

\begin{tabular}{|c|c|c|c|}
\hline Radionuclides & Physical half-life & $\begin{array}{l}\text { Principle gamma photon radiation } \\
\text { emission(s) }\end{array}$ & $\begin{array}{l}\text { Emission probability per decay } \\
\text { (percent photon yield) }\end{array}$ \\
\hline Cobalt-57 ( $\left.{ }^{57} \mathrm{Co}\right)$ & 271.8 days & $14,122,136 \mathrm{keV}$ & $9.2,85.5,10.7 \%$ \\
\hline Fluorine- $18\left({ }^{18} \mathrm{~F}\right)$ & 110 minutes & $5 \mathrm{II} \mathrm{keV}^{*}$ & $19.3 \%$ \\
\hline Galium-67 ( $\left.{ }^{67} \mathrm{Ga}\right)$ & 78.3 hours (3.26 days) & $91,93,184,209,300,393 \mathrm{keV}$ & $3.0,37.8,20.1,2.4,16.8,4.7 \%$ \\
\hline Indium-III (IIIIn) & 67.4 hours ( $2.8 \mathrm{I}$ days) & I7I, $247 \mathrm{keV}$ & $90.7,94.1 \%$ \\
\hline lodine-| 23 (123|) & 13.2 hours & $159,529 \mathrm{keV}$ & $83.4,1.3 \%$ \\
\hline lodine- 124 ( $\left.{ }^{124} \mid\right)$ & 100.3 hours (4.18 days) & $5 \mathrm{II} \mathrm{keV} *$ & not easily characterized \\
\hline lodine-125 (125|) & I 443.4 hours ( 60.14 days) & $35 \mathrm{keV}$ & $6.7 \%$ \\
\hline lodine-|3| (13| $\mid)$ & 193.0 hours (8.04 days) & $80,284,364,637,642,723 \mathrm{keV}$ & $2.6,6.1,81.2,7.3,0.2,1.8 \%$ \\
\hline Technetium-99m (99mTc) & 6.04 hours & $140,142 \mathrm{keV}$ & $88.5,0.023 \%$ \\
\hline Thallium-20I (20ITI) & 73.0 hours (3.04 days) & $7 \mathrm{I}, 135,167 \mathrm{keV}$ & $47.0,2.7,10.0 \%$ \\
\hline
\end{tabular}

* The $5 \mathrm{II}$ keV gamma photons are generated from positron-electron annihilation.

general, the gamma photon radiation emitted from each radionuclide, which is expressed in kiloelectron volts $(\mathrm{keV})$, can be characterized as low-energy emission $(0 \mathrm{keV}$ to $150 \mathrm{keV})$, medium-energy emission $(150 \mathrm{keV}$ to 400 $\mathrm{keV}$ ), or high-energy emission (greater than $400 \mathrm{keV}$ ). To date, the radionuclides that have been utilized most frequently with the gamma detection probe for the specific application of radioguided surgery have been ${ }^{125} \mathrm{I},{ }^{111} \mathrm{In}$,

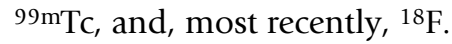

\section{Radionuclides of iodine}

Four radionuclides of iodine have been utilized in radioguided surgery, including ${ }^{123} \mathrm{I},{ }^{124} \mathrm{I},{ }^{125} \mathrm{I}$, and ${ }^{131} \mathrm{I}$ $[26,28,29]$. In this regard, various radiopharmaceutical agents have been developed using radionuclides of iodine in conjunction with monoclonal antibody carriers as well as receptor-specific carriers and tissue-specific carriers.

By far, ${ }^{125}$ I has been utilized most frequently in the past in the form of a radiolabeled conjugate with various monoclonal antibodies for gamma probe detection of tumor in radioguided surgery $[26,28,30] .{ }^{125}$ I has a relatively long physical half-life of approximately 60 days and possesses an extremely low gamma photon emission energy of 35 $\mathrm{keV}$. Generally speaking, ${ }^{125} \mathrm{I}$ is not suitable for diagnostic nuclear medicine imaging due to its low gamma photon emission energy, which results in weak tissue penetration and high soft tissue attenuation, and a resultant poor image quality. Instead, diagnostic gamma camera imaging is more ideally suited for radionuclides with gamma photon emission energies in the $100 \mathrm{keV}$ to $200 \mathrm{keV}$ range. However, the low gamma photon emission energy and high soft tissue attenuation of ${ }^{125}$ I is highly advantageous in gamma probe detection of tumor in radioguided surgery, since the principle of gamma probe detection generally relies on close approximation of the gamma detection probe to the source of the radioactivity for the facilitation of accurate tumor detection. Additionally, the long physical half-life of ${ }^{125}$ I has been shown to be advantageous for gamma probe detection of tumor in radioguided surgery involving whole monoclonal antibodies due to the prolonged time of approximately 14 to 21 days that its takes for such ${ }^{125}$ I-radiolabeled whole monoclonal antibody conjugates to reach optimal pharmacokinetics and to accomplish maximal tumor localization with maximum background washout.

${ }^{131}$ I has a physical half-life of approximately 8 days and was the first radionuclide used in the radiolabeling of monoclonal antibodies $[26,28,30]$. The principle gamma photon emission energy of ${ }^{131}$ I that is utilized in nuclear medicine is that of the $364 \mathrm{keV}$ gamma photon. The highly energetic nature of these $364 \mathrm{keV}$ gamma photons increase background counts secondary to scatter and resultantly complicates the tumor detection efficiency of gamma probe detection of tumor during radioguided surgery. Likewise, these highly energetic $364 \mathrm{keV}$ gamma photons require high-energy collimation and are generally less well-detected by diagnostic gamma camera imaging secondary to the limited stopping ability of the crystal element within the diagnostic gamma camera imaging device. The beta particulate emissions of ${ }^{131}$ I contribute significantly to the absorbed dose of radiation to the patient, thus limiting the amount of ${ }^{131}$ I dose that can be administered to the patient. The utilization of ${ }^{131} \mathrm{I}$ has principally been limited to that of therapeutic applications for obliteration of thyroid tissue and for radioguided surgery for guiding the resection of recurrent thyroid cancer after diagnostic imaging.

${ }^{123}$ I has a physical half-life of approximately 13 hours, has a principle gamma photon emission energy of $159 \mathrm{keV}$, and has a relative absence of beta particulate emissions $[26,28,30]$. These features allow one to administer relatively larger doses of ${ }^{123} \mathrm{I}$ dose to patients relative to other radionuclides of iodine. Likewise, these features make ${ }^{123}$ I relatively ideal for detection by diagnostic gamma camera imaging with a number of different carrier agents, includ- 
ing metaiodobenzylguanidine (MIBG), since diagnostic gamma camera imaging is most ideally suited for radionuclides with gamma photon emission energies in the 100 $\mathrm{keV}$ to $200 \mathrm{keV}$ range. Additionally, ${ }^{123}$ I-labeled MIBG has been successfully used in gamma probe detection during radioguided surgery. However, the short physical half-life of approximately 13 hours for ${ }^{123}$ I makes it somewhat unsuitable for radioiodination of ${ }^{123}$ I to whole monoclonal antibodies for use in gamma probe detection during radioguided surgery, since such ${ }^{123}$ I-labeled whole monoclonal antibody conjugates may take many days to reach optimal pharmacokinetics and to accomplish maximal tumor localization with accompanying maximum background washout. However, ${ }^{123}$ I labeled monoclonal antibody fragments have been used in diagnostic gamma camera imaging.

${ }^{124}$ I has a physical half-life of approximately 4 days [3133]. The emission spectrum and decay schema for ${ }^{124} \mathrm{I}$ is very complex and is beyond the scope of this review to fully characterize. Only about $23 \%$ of disintegrations from ${ }^{124}$ I result in positron emissions, and these are generally of relatively high energy [32]. There are also numerous high-energy gamma photon emissions that occur, some of which occur in cascade with the positron emissions [32]. ${ }^{124}$ I has been used primarily in diagnostic positron emission tomography (PET) imaging [32,34], but is currently under investigation in radioguided surgery with gamma detection probes and PET probes (measuring both high-energy gamma photon emissions and beta emissions) [29].

\section{IIIIn}

${ }^{111}$ In has a physical half life of approximately 2.8 days, has two principle gamma photon emissions with energies of $171 \mathrm{keV}$ and $247 \mathrm{keV}$, and has a relative absence of beta particulate emissions $[26,28]$. These features make ${ }^{111}$ In a relatively ideal radionuclide for detection by diagnostic gamma camera imaging in conjunction with a number of different carrier agents. However, the propensity of ${ }^{111}$ Incontaining radiopharmaceuticals, such as ${ }^{111}$ In-labeled monoclonal antibodies, to accumulate within the reticuloendothelial system (such as the liver, spleen, and bone marrow) result in relatively high background counts and can limit its potential usefulness for gamma probe detection of tumor during radioguided surgery.

\section{$99 m$ Tc}

${ }^{99 \mathrm{~m} T \mathrm{Tc}}$ has a physical half-life of approximately 6 hours, has a principle gamma photon emission energy of 140 $\mathrm{keV}$, and has a relative absence of beta particulate emissions $[26,28]$. ${ }^{99 \mathrm{mTc}}$ remains the leading radionuclide imaging agent used in diagnostic nuclear medicine secondary to several desirable characteristics, including: (1) the principle gamma photon emission energy of $140 \mathrm{keV}$ that is ideal both for detection by diagnostic gamma camera imaging and for gamma probe detection of tumor and identification of SLNs during radioguided surgery; (2) a low patient-absorbed radiation dose; (3) a low cost per patient dose; and (4) widespread commercial availability.

${ }^{18} \mathrm{~F}$

A radionuclide to more recently gain interest for radioguided surgery has been ${ }^{18} \mathrm{~F}$ [35-54]. ${ }^{18} \mathrm{~F}$ has a relatively short physical half-life of approximately 110 minutes $[55,56]$. The radioactive decay of ${ }^{18} \mathrm{~F}$ is predominantly $(97 \%)$ by positron (positively charged electron) emission. The maximum positron radiation emission energy of ${ }^{18} \mathrm{~F}$ is 635 $\mathrm{keV}$, giving ${ }^{18} \mathrm{~F}$ a relatively low positron radiation emission energy. As a result, the positron emitted from the nucleus of this proton-rich/neutron-deficient radionuclide can travel only a short distance (approximately 2 millimeters) within the biological tissue before it interacts (collides) with a negatively charged electron. It is the interaction (collision) of the emitted positron from the ${ }^{18} \mathrm{~F}$ nucleus with a negatively charged electron within the biological tissue and the resultant positron-electron annihilation within the biological tissue that then generates two high-energy $511 \mathrm{keV}$ gamma photons. Therefore, the resultant detection of ${ }^{18} \mathrm{~F}$ during radioguided surgery can occur by one of two mechanisms: (1) a direct mechanism of detection of positrons (beta particulate emissions) by a positron detection probe or (2) an indirect mechanism of detection of high-energy $511 \mathrm{keV}$ gamma photons arising from positron-electron annihilation by a gamma detection probe. These highly energetic $511 \mathrm{keV}$ gamma photons, that are the basis of the indirect mechanism of detection by a gamma detection probe, can result in relatively high background counts and can potentially complicate tumor detection efficiency of the gamma detection probe during radioguided surgery.

\section{Radiopharmaceutical agents utilized in radioguided surgery}

Radioguided surgery using gamma detection probe technology has undergone an ever-changing evolution with regards to which radiopharmaceutical agents have been most frequently employed [49]. In the early years of gamma probe detection in radioguided surgery, the radiopharmaceutical agents most frequently utilized were radionuclides of iodine that were labeled to various monoclonal antibodies. More recently, with the advent of radioguided SLN biopsy technology and its application to numerous surgically managed malignancies, the use of $99 \mathrm{mTc}$-labeled radiopharmaceutical agents with the gamma detection probe in radioguided surgery has increased dramatically, and, at the present time, accounts for the vast majority of the radioguided surgical procedures performed. However, future directions for the gamma probe in radioguided surgery are currently being 
evaluated and the use of ${ }^{18} \mathrm{~F}$-fluorodeoxyglucose ${ }^{18} \mathrm{~F}-$ FDG) as a radiopharmaceutical agent for gamma probe detection of tumor in radioguided surgery holds exciting promise $[46,49]$.

\section{Monoclonal antibodies and their tumor-associated antigens}

The specific application of monoclonal antibodies to radioguided surgery has been the basis for, and has represented the most important component to, the development of the radioimmunoguided surgery (RIGS) system [30,57]. This system was pioneered at The Ohio State University in the early 1980 s by the collaboration of a surgical oncologist, Dr. Edward W. Martin, Jr., and a professor emeritus of electrical engineering, Dr. Marlin O. Thurston [58,59].

The production of a monoclonal antibody is the result of a technique called hybridoma fusion technology [60]. Most simply stated, a B-cell lymphocyte (which recognizes a single particular antigen and subsequently produces a single antibody targeting that specific antigen) and a myeloma cell are fused together to create a hybridoma cell. This immortalized hybridoma cell has the ability to survive and replicate outside of the animal. Such a hybridoma cell is able to replicate and be maintained in cell culture and will produce large amounts of a single antibody, which is referred to as a monoclonal antibody.

Monoclonal antibodies used in RIGS can be targeted against antigens expressed on the surface of tumor cells or targeted against antigens expressed within the extracellular environment around tumor cells $[30,49,57]$. When radiolabeled with various radionuclides, such resulting radiolabeled monoclonal antibody conjugates can potentially be utilized in both diagnostic gamma camera imaging and gamma probe detection of tumors, as well as in cancer therapeutics. In this regard, both whole monoclonal antibodies and monoclonal antibody fragments have been investigated.

The most advantageous features of an ideal monoclonal antibody are: (1) high affinity for its antigen (i.e., the initial ability to bind to the antigen); (2) high avidity for its antigen (i.e., the ability of the antibody to remain bound over an extended period of time); (3) rapid penetration into the tumor tissue; (4) rapid clearance from the bloodstream; (5) minimal accumulation within normal tissues; and (6) the absence of a human antimouse antibody (HAMA) response $[8,17,30,58,59,61]$.

Nevertheless, the production of radiolabeled monoclonal antibody is not necessarily a simple endeavor $[26,30]$. The conjugation of a radionuclide to a monoclonal antibody may potentially change the specific binding properties of the monoclonal antibody. In such an instance in which the specific binding properties of the monoclonal antibody are significantly altered, the resultant radiolabeled monoclonal antibody may be left with significantly reduced affinity and/or avidity for the intended target antigen that ultimately renders the resultant radiolabeled monoclonal antibody clinical ineffectual.

The particular form of the monoclonal antibody (i.e., whether it is a whole monoclonal antibody or a fragment of a monoclonal antibody) can influence its ability to localize tumor [30]. Monoclonal antibody fragments have smaller molecular weight, have more rapid penetration into tumors, and have more rapid clearance rate from the bloodstream. As a result, the use of radiolabeled monoclonal antibody fragments can result in lower normal tissue background activity and lead to increased tumor to background ratio and improved tumor detections. However, monoclonal antibody fragments tend to accumulate more within the kidneys and, as a result, they may not be useful in the evaluation of the tumors within or around the area of the kidneys or the bladder.

Numerous radiolabeled monoclonal antibodies have been clinically investigated for radioimmunodetection and in RIGS $[30,49]$. The most intensely investigated and clinically evaluated monoclonal antibodies have been those directed against tumor-associated glycoprotein-72 (TAG-72), carcinoembryonic antigen (CEA), and tumorassociated antigen 17-1A. Several generations of antiTAG-72 monoclonal antibodies have been developed, including two murine-derived anti-TAG-72 monoclonal antibodies (B72.3, native murine CC49) and one humanized anti-TAG-72 monoclonal antibody (HuCC49).

TAG-72 is a tumor-associated glycoprotein with a molecular weight of greater than 10 million Daltons $[62,63]$. TAG-72 contains approximately $80 \%$ carbohydrates, has mucin-like biochemical and biophysical properties similar to colonic, small intestine, and gastric mucins, and is thought to be secreted by epithelial tissues $[62,63]$. Numerous epithelial-derived cancers, including colorectal, breast, gastric, pancreatic, ovarian, and non-small cell lung cancers overexpress TAG-72 [62,64]. TAG-72 is predominantly located within mucin pools of the extracellular environment around the tumor cells and is not specifically expressed on the tumor cell surface. Of particular importance, TAG-72 has been shown to be associated with over $90 \%$ of the colorectal, gastric, and ovarian carcinomas and in approximately $70 \%$ of breast carcinomas [65-68]. Finally, while it is rarely expressed in normal human adult tissues or in benign disease processes, TAG72 is also expressed in some normal human fetal tissues, including normal fetal intestine [69]. 
B72.3 was the first-generation murine anti-TAG-72 monoclonal antibody that was developed and was interestingly first derived from reaction with human mammary tumor cells [70]. B72.3 was shown to be reactive with a variety of human carcinomas, including colorectal (94\%), breast ( $84 \%$ of invasive ductal), ovarian (100\% of common epithelial), as well as the majority of gastric, pancreatic, endometrial, and lung adenocarcinomas [30,65,67$69,71]$. In contrast, B72.3 was shown to have only a very weak or a nonreactivity status to a variety of normal adult human tissues [30]. The only exception to this rule has been demonstrated for normal postovulatory (secretory phase) endometrium which was shown to be reactive to B72.3, in contrast to normal preovulatory (proliferative phase) endometrium which was nonreactive $[30,71]$.

Native murine CC49 was the second-generation murine anti-TAG-72 monoclonal antibody that was developed $[30,63,72,73]$. Native murine CC49 was found to have only minimal reactivity to a variety of normal human tissues, recognized a different epitope on the TAG-72 as compared to B72.3, and exhibited higher reactivity than B72.3 to a variety of human carcinomas, including colorectal, breast, ovarian, and lung carcinomas [30,72,73]. From a clinical perspective and as will later be discussed in the clinical application section, native murine CC49 was also superior to B72.3 in tumor detection in RIGS for colorectal carcinoma $[74,75]$.

It is well characterized that a majority of patients will develop some degree of a HAMA response to the administration of murine monoclonal antibodies [30,64,76-78]. Despite the fact that the HAMA response has been well characterized, its clinical impact on cancer patients, whether deleterious or beneficial, remains very unclear $[79,80]$. Nevertheless, in order to attempt to eliminate this antiimmunoglobulin response, a third-generation humanized anti-TAG-72 monoclonal antibody (HuCC49) was genetically engineered [81]. HuCC49 demonstrated equivalent tumor-targeting for human colon carcinoma xenografts but a tradeoff of slightly less relative affinity to TAG-72 as compared to native murine CC49 and chimeric CC49 [81]. However, HuCC49 was shown to not produce a HAMA response [82]. Further refinements were made in HuCC49 by the development of a higher affinity HuCC49 possessing a $\mathrm{CH} 2$ domain deletion (i.e., HuCC49 $\Delta \mathrm{C}_{\mathrm{H}} 2$ ) [83]. HuCC $49 \Delta \mathrm{C}_{\mathrm{H}} 2$ demonstrated a more rapid blood clearance, a higher affinity constant $\left(5.1 \times 10^{-9}\right.$ versus $\left.2.1 \times 10^{-9}\right)$, and significantly lower percent of the injected dose in normal tissues compared to intact HuCC49 [83], thus indicating the potential utility of the HuCC $49 \Delta \mathrm{C}_{\mathrm{H}} 2$ monoclonal antibody for diagnostic and therapeutic clinical applications. Furthermore, population pharmacokinetic modeling studies have demonstrated that HuCC $49 \Delta \mathrm{C}_{\mathrm{H}} 2$ had more rapid clearance (65\% increase) from bloodstream and a resultant shorter "residence time" (24\% shorter) than that of native murine CC49 [84].

Carcinoembryonic antigen (CEA) represents another well-studied and potentially useful target antigen for which radiolabeled monoclonal antibodies have been developed and investigated for RIGS [30]. CEA is a tumorassociated glycoprotein with a molecular weight of approximately 200,000 Daltons [85,86]. It is highly expressed on the cell surface of both embryonic colonic mucosa as well as a wide range of human adenocarcinomas, including colorectal, gastric, pancreatic, breast, ovarian, endometrial, and lung [30,85-87]. Specific to colorectal adenocarcinomas, it has been previously reported that anywhere from $66 \%$ to $100 \%$ express CEA [30].

Numerous murine monoclonal antibodies have been developed to target CEA $[30,85,88-93]$. Those most well studied have included COL-1, $\mathrm{A}_{5} \mathrm{~B}_{7}$, IMMU-4, and CL58. COL-1 monoclonal antibody was first derived from reaction with LS-174T human colon carcinoma xenograft in athymic mice, has a very high affinity to CEA, and has been shown to have a high reactivity to significant number of colon, breast, and lung carcinomas $[30,85,88,89]$. Likewise, $A_{5} B_{7}$, IMMU-4, and CL58 represent three additional anti-CEA murine monoclonal antibodies that have shown clinical relevance by possessing a high reactivity to CEA-producing malignancies $[30,88,90$ 93].

Lastly, 17-1A (also called EpCAM) is a tumor-associated glycoprotein with a molecular weight in the range of approximately 30, 000 to 40,000 Daltons [94-96] which is thought to represent a cell-cell adhesion molecule. It was first characterized on a human colorectal adenocarcinoma cell line SW1083 [97]. It is broadly distributed in normal epithelial tissues and in various carcinomas, including colorectal, gastric, and breast [94,95,98].

Murine monoclonal antibodies against the tumor-associated antigen 17-1A were originally developed in the hybridoma SW1083-17-1A $[57,99,100]$. The localization and clearance properties of the 17-1A murine monoclonal whole antibody and its monoclonal antibody fragment were previously evaluated in a mice xenograft model by Martin et al [101], demonstrating high tumor-to-normal tissue ratios with highest tumor-to-normal tissue ratios seen at 72 hours and 24 hours, respectively, for the 17-1A murine monoclonal whole antibody and monoclonal antibody fragment $[57,101]$.

The most common challenges facing the utility of monoclonal antibodies in radioimmunodetection relate to the 
activity ratio between tumor and normal surrounding tissues and the time interval between the initial administration of the radiopharmaceutical agent and performance of diagnostic gamma camera imaging or radioguided surgical detection. In an attempt to increase the activity ratio between tumor and normal surrounding tissues and to decrease the time interval between the initial administration of the radiopharmaceutical agent and performance of diagnostic gamma camera imaging or radioguided surgical detection, pretargeting strategies for monoclonal antibodies and radionuclides have been investigated [102]. Most such pretargeting strategies utilize the principle of the avidin-biotin binding system. This avidin-biotin pretargeting strategy allows for the complete temporal separation of the systemic administration of the monoclonal antibody from that of the systemic administration of the radionuclide. The monoclonal antibody is labeled with biotin and the radionuclide is labeled with avidin. This will ultimately result in a reduction of nonspecific binding. The biotin-labeled monoclonal antibody is first administered, allowing binding of the biotin-labeled monoclonal antibody to the tumor and allowing the nonspecific uptake of the biotin-labeled monoclonal antibody to be cleared. The avidin-labeled radionuclide is then administered and resultantly localizes in the tumor secondary to the high affinity and specificity of the avidinlabeled radionuclide for the biotin-labeled monoclonal antibody. More recently, an additional pretargeting strategy utilizing a bispecific antibody and radiolabeled bivalent hapten system has been investigated that bind cooperatively to target cells [103].

\section{Radioactive iodine-labeled radiopharmaceutical agents}

The vast majority of radioactive iodine-labeled radiopharmaceutical agents that have been utilized with the gamma detection probe for tumor detection in radioguided surgery have been those radionuclides of iodine that have been labeled to various monoclonal antibodies [30]. The predominant iodine radionuclide that has been labeled to various monoclonal antibodies and utilized with the gamma detection probe for tumor detection in radioguided surgery has been ${ }^{125} \mathrm{I}$, and to a much lesser degree ${ }^{131}$ I. The radiolabeling of ${ }^{123}$ I to monoclonal antibodies has not been proven useful for tumor detection in radioguided surgery for the reasons previously discussed. Both ${ }^{131} \mathrm{I}$ and ${ }^{123} \mathrm{I}$ are used with MIBG, a molecule similar to norepinephrine, for identification of neuroendocrine tumors.

\section{$99 \mathrm{~m}$ Tc-labeled radiopharmaceutical agents}

Numerous 99mTc-labeled radiopharmaceutical agents have been formulated for use in diagnostic nuclear medicine by radiolabeling the radionuclide $99 \mathrm{~m}$ Tc to various compounds $[26,104]$. The list of compounds that have been radiolabeled with $99 \mathrm{~m} T \mathrm{Tc}$ for diagnostic nuclear med- icine use is extensive and includes, in alphabetical order, antimony trisulfide colloid, bicisate dihydrochloride, colloidal human albumin (i.e., nanocolloid), colloidal rhenium sulfide, dextran, diethylenetriaminepentaacetic acid (DTPA)-mannosyl-dextran, disofenin, hydroxyl-ethyl starch, exametazime, gluceptate, glucoheptonate, hexakis2-methoxy-isobutyl-isonitrile (methoxyisobutylisonitrile, MIBI, or sestamibi), hydroxymethylene diphosphonate (HMDP or oxidronate), hydroxyethylene diphosphonate (HDP), lidofenin, mebrofenin, mertiatide (mercaptoacetylglyclyglyclyglycine), methylene diphosphonate (MDP or medronate), pentetate (diethylenetriaminepentaacetic acid), sodium pertechnetate, sodium phytate (D-myo-inositol 1,2,3,4,5,6-hexakisphosphate dodecasodium), sodium pyrophosphate, stannous phytate, succimer, sulfur colloid, teboroxime, tetrofosmin, and tin colloid. The primary ${ }^{99 m}$ Tc-labeled radiopharmaceutical agents that have been used for radioguided SLN biopsy include 99mTc sulfur colloid, 99mTc colloidal human albumin, and 99mTc antimony trisulfide colloid. The primary $99 \mathrm{~m}$ Tc-labeled radiopharmaceutical agents that have been used for tumor detection during radiogu-

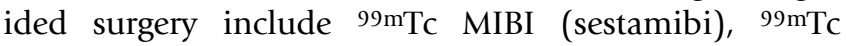
diphosphonates, and ${ }^{99 \mathrm{mTC}}$ sodium pertechnetate. The application of 99mTc-labeled monoclonal antibody fragments, such as ${ }^{99 \mathrm{mTC}-l a b e l e d}$ arcitumomab (IMMU-4 murine monoclonal antibody fragments against CEA) and $99 \mathrm{mTc}$-nofetumomab merpentan (monoclonal antibody fragment of the pancarcinoma murine antibody NRLU-10) have been used in nuclear medicine imaging but have only been very limitedly investigated for tumor detection during radioguided surgery [105-107].

\section{I'In-labeled radiopharmaceutical agents}

Several 111In-labeled radiopharmaceutical agents have been formulated for use in diagnostic nuclear medicine by radiolabeling the ${ }^{111}$ In with various compounds $[26,30,108,109]$. This includes the ${ }^{111}$ In-labeled somatostatin analogue, ${ }^{111}$ In-diethylenetriaminepentaacetic acidD-phenylalanine ${ }^{1}$-octreotide (111In-DTPA-D-Phe ${ }^{1}$-octreotide or ${ }^{111}$ In-pentetreotide), as well as various ${ }^{111}$ Inlabeled monoclonal antibodies. ${ }^{111}$ In-(DTPA)-D-Phe ${ }^{1}$ octreotide binds to somatostatin receptors, predominantly of somatostatin receptor subtype sst 2 and sst 5 , and have been useful for diagnostic nuclear medicine imaging of neuroendocrine tumors and of non-neuroendocrine tumors which express somatostatin receptors [26,108]. Likewise, ${ }^{111}$ In-labeled monoclonal antibodies have been investigated in colorectal cancer $[30,109]$. However, ${ }^{111}$ Inlabeled monoclonal antibodies have been of somewhat limited usefulness secondary to the previously discussed nonspecific accumulation of ${ }^{111}$ In within reticuloendothelial organs, such as within the liver and spleen. This resultant nonspecific binding generally interferes with the detection of tumor within or around the liver and spleen 
during radioguided surgery and is therefore undesirable $[26,30]$.

\section{${ }^{18} \mathrm{~F}$-fluorodeoxyglucose (18F-FDG)}

Malignant tumors have long been known to have an accelerated rate of glucose metabolism and have an increased rate of glucose transport and glucose utilization [110112]. The mechanism of ${ }^{18}$ F-FDG within malignant cells is well described in the literature [113-115]. ${ }^{18} \mathrm{~F}-\mathrm{FDG}$ is an ${ }^{18} \mathrm{~F}-$-labeled nonphysiologic analog of glucose. ${ }^{18} \mathrm{~F}-\mathrm{FDG}$ within the bloodstream is transported into cells (both malignant cells and normal cells) by a facilitated diffusion mechanism involving specific glucose transporters (i.e., GLUT transporters). Once within the cell, ${ }^{18} \mathrm{~F}-\mathrm{FDG}$ is phosphorylated to ${ }^{18}$ F-FDG-6-phosphate by the enzyme hexokinase. Unlike ${ }^{18}$ F-FDG, ${ }^{18}$ F-FDG-6-phosphate can not be readily transported across the cellular membrane of either malignant cells or normal cells. The enzyme glucose-6-phosphatase is responsible for dephosphorylating ${ }^{18} \mathrm{~F}-\mathrm{FDG}-6$-phosphate to ${ }^{18} \mathrm{~F}-\mathrm{FDG}$. Because the enzyme glucose-6-phosphatase is present in relatively low amounts within malignant cells and within normal cells, ${ }^{18}$ F-FDG-6-phosphate cannot be readily dephosphorylated back to ${ }^{18}$ F-FDG once ${ }^{18}$ F-FDG has been phosphorylated within the intracellular environment. Therefore, once ${ }^{18} \mathrm{~F}-\mathrm{FDG}$ is transported into the malignant cell or the normal cell via the GLUT transporters and is subsequently phosphorylated, the resultant ${ }^{18} \mathrm{~F}-\mathrm{FDG}-6$-phosphate is essentially trapped within the cell. Additionally, ${ }^{18} \mathrm{~F}-\mathrm{FDG}-$ 6-phosphate cannot be utilized in the metabolic steps of glycolysis, and, this further lends to the accumulation of ${ }^{18}$ F-FDG-6-phosphate within the cell. This entire process is thought to occur more readily in malignant cells than in normal cells due to the overexpression of the glucose transporters GLUT 1 and GLUT 3 by malignant cells and due to higher levels of hexokinase within malignant cells. The overall result of this entire process of an accelerated rate of glucose metabolism and an increased rate of glucose transport and glucose utilization by malignant cells is that of a relatively greater accumulation of 18 F-FDG-6phosphate within malignant cells as compared to normal cells. Even more simply stated, malignant cells are much more efficient at accumulating glucose molecules within their intracellular environment than are normal cells. This elegantly elucidated process represents the overall basis for the clinical application of 18 F-FDG for the detection of tumor by both diagnostic PET imaging and gamma detection probe technology.

However, limitations do exist in regards to the utilization of ${ }^{18} \mathrm{~F}-\mathrm{FDG}$ in both diagnostic PET imaging and gamma detection probe technology. These limitations are: (1) the accumulation of ${ }^{18} \mathrm{~F}$-FDG within certain normal tissues with an elevated rate of glucose metabolism (most striking in the brain and heart, and to a lesser degree in the mucosa and smooth muscle of the stomach, small intestine and colon, as well as in thyroid, liver, spleen, and skeletal muscle); (2) the accumulation of ${ }^{18}$ F-FDG within in inflammatory/granulomatous processes and infectious processes; (3) the excretion and accumulation of ${ }^{18} \mathrm{~F}-\mathrm{FDG}$ within the urinary tract (kidneys, ureters, and bladder); and (4) the impaired uptake of ${ }^{18} \mathrm{~F}-\mathrm{FDG}$ in patients with elevated blood glucose levels and with impaired glucose metabolism [113,116,117].

\section{Occupational radiation exposure from radiopharmaceutical agents utilized during radioguided surgery}

The assessment of occupational radiation exposure to surgical personnel involved in radioguided surgical procedures is important to maintaining a safe work environment for such personnel. This has been evaluated

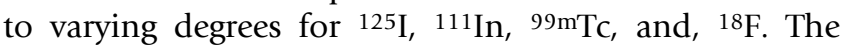
United States Nuclear Regulatory Commission (USNRC) has set the annual occupational exposure limit for adults as a total effective dose equivalent of $50,000 \mu \mathrm{Sv}$ [118]. The International Commission on Radiological Protection (ICRP) has set the annual occupational exposure limit for adults as a total effective dose equivalent of $20,000 \mu \mathrm{Sv}$ per year, averaged over a five year period $(100,000 \mu \mathrm{Sv}$ in five years), with further provision that the total effective dose equivalent should not exceed 50,000 $\mu \mathrm{Sv}$ in any single year $[119,120]$.

For ${ }^{125}$ I-labeled monoclonal antibodies, the radiation exposure to the surgeon has been previously assessed during RIGS $[78,121]$. For a mean dosage of $2 \mathrm{mCi}(74 \mathrm{MBq})$ of ${ }^{125}$ I that was radiolabeled to $1 \mathrm{mg}$ of B72.3 monoclonal antibody and was injected at a mean of 23.4 days prior to surgery, the mean dose equivalent for the surgeon was determined to be only $0.2 \mu \mathrm{Sv}$ per hour of exposure and was not significantly different from that of the environmental (operating room) background.

For ${ }^{111}$ In radiopharmaceuticals, no data on radiation exposure to surgical personnel is currently available. However, in this regard, limited data from one study reported that staff members and technologists involved in treating patients with somatostatin receptor positive tumors with a therapeutic dosage ( $216 \mathrm{mCi}$ or $8000 \mathrm{MBq}$ ) of an ${ }^{111}$ In-labeled somatostatin analogue received a mean whole body dose equivalent of only $45 \mu \mathrm{Sv}$ per case [122].

Radiation exposure to surgical personnel members from 99mTc-labeled radiopharmaceutical agents used for radioguided SLN biopsy has been well-documented [123127]. There was significant variability in the whole body dose equivalent incurred by the surgeon and this value was highly dependent upon the injection dose of the 
99mTc-labeled radiopharmaceutical agent and the total duration of time from the injection to the start of the radioguided SLN biopsy procedure. On one hand, Waddington et al reported mean whole body dose equivalent of only $0.34 \mu \mathrm{Sv}$ per case when $0.27 \mathrm{mCi}(10 \mathrm{MBq})$ to 0.41 $\mathrm{mCi}(15 \mathrm{MBq})$ of $99 \mathrm{mTc}$ colloidal albumin was injected 24 hours prior to the start of the radioguided SLN biopsy procedure [124]. On the other hand, Stratmann et al reported a mean whole body dose equivalent of $13.3 \mu \mathrm{Sv}$ per case when $0.7 \mathrm{mCi}(26 \mathrm{MBq})$ to $1.1 \mathrm{mCi}(41 \mathrm{MBq})$ of $99 \mathrm{mTc}$ sulfur colloid was injected 90 to 180 minutes prior to the start of the radioguided SLN biopsy procedure [123]. Nevertheless, the data supports the fact that the whole body dose equivalent incurred by a surgeon during any given radioguided SLN biopsy procedure using a $99 \mathrm{mTC}$-labeled radiopharmaceutical agent is extremely low.

Radiation exposure to surgical personnel members from 99mTc-MIBI used for radioguided brain tumorectomy has been previously investigated [128]. The dosage of 99mTcMIBI that was intravenously administered on the day of surgery was not specified. The mean exposure time was 6.1 hours for all surgical personnel members. The mean whole body dose equivalent per case was 27.9, 25.8, and $14.9 \mu \mathrm{Sv}$, respectively, for the surgeon, nurse, and anesthetist. More recently, Bekiş et al [129] evaluated radiation exposure to surgical staff from $99 \mathrm{mTc}$-MIBI used in two cases of radioguided parathyroidectomy. When $20 \mathrm{mCi}$ (740 MBq) of Tc-99m MIBI was intravenously injected 3 hours prior to the operation and with a mean operative time of 83 minutes, the least and highest exposure of the surgical staff were calculated as 1.01 and $3.63 \mu \mathrm{Sv}$ for the anesthetist, 8.78 and $11.00 \mu \mathrm{Sv}$ for the senior surgeon, 7.60 and $10.00 \mu \mathrm{Sv}$ for the first assistant surgeon, 6.75 and $8.20 \mu \mathrm{Sv}$ for the second assistant surgeon, and 3.64 and $8.00 \mu \mathrm{Sv}$ for the nurse.

The issue of radiation exposure to intraoperative and perioperative personnel involved in radioguided surgery cases utilizing ${ }^{18} \mathrm{~F}-\mathrm{FDG}$ has become a topic of recent interest. However, data on this topic is currently very limited $[42,47,50,51,130-132]$. In a recent comprehensive evaluation, our group at The Ohio State University has determined that after a mean dosage of $18.9 \mathrm{mCi}(699 \mathrm{MBq})$ of ${ }^{18} \mathrm{~F}-\mathrm{FDG}$ injected at a mean of 142 minutes prior to surgery that the mean deep dose equivalent per case was 164 , $119,92,63,54$, and $48 \mu \mathrm{Sv}$, respectively, for the surgeon, anesthetist, scrub technologist, postoperative nurse, circulating nurse, and preoperative nurse [132]. This data clearly illustrates that the absorbed radiation dose received by both intraoperative and perioperative personnel involved in ${ }^{18} \mathrm{~F}-\mathrm{FDG}$ radioguided surgery cases is relatively low per case and allows for all these personnel to participate in multiple such cases and still remain well below standards set for occupational exposure limits.

\section{Clinical applications (Table 2) Breast cancer}

There are numerous reported applications of gamma detection probe technology during radioguided surgery for breast cancer. However, by far, the single most important and most widely utilized application of the gamma detection probe in breast cancer surgery has been for radioguided SLN biopsy.

\section{Radioguided SLN biopsy}

It is clearly evident that SLN biopsy has become widely accepted as a standard of care in the surgical staging of the axillary lymph nodes during breast cancer surgery $[133,134]$. The intraoperative use of the gamma detection probe for radioguided SLN biopsy in breast cancer was first described in 1993 by Krag et al [135] at The University of Vermont (Table 1). Since that time, over 3,300 articles have been published which have been sited in PUBMED under the search descriptors of "breast cancer" and "sentinel lymph node".

Worldwide, numerous $99 \mathrm{mTc}$-based agents have been utilized for radioguided SLN biopsy for breast cancer $[133,136]$. This includes ${ }^{99 \mathrm{~m} T c}$ sulfur colloid, ${ }^{99 \mathrm{~m} T \mathrm{Tc} \text { anti- }}$ mony trisulfide colloid, $99 \mathrm{mTc}$ colloidal human albumin

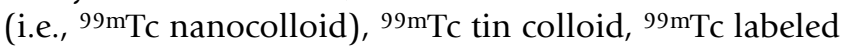
dextran, ${ }^{99 \mathrm{~m} T c}$ hydroxyl-ethyl starch, and $99 \mathrm{mTc}$ stannous phytate. However, within the United States, ${ }^{99 \mathrm{~m} T c}$ sulfur colloid represents the only registered and licensed radiocolloid for use SLN biopsy. The dosing of these radiocolloid agents varies considerably within the literature and have been reported as low as $0.1 \mathrm{mCi}(3.7 \mathrm{MBq})$ [137] and as high as $10 \mathrm{mCi}$ (370 MBq) [138]. Generally, in current practice, these radiocolloid agents are most commonly administered on the day of surgery in a dosing range from $0.4 \mathrm{mCi}(14.8 \mathrm{MBq})$ to $1 \mathrm{mCi}$ (37 MBq) [133]. These radiocolloid agents are generally administered one to six hours prior to the planned breast cancer surgery, although prior-day injection of radiocolloid has been shown to be technically feasible $[137,139,140]$.

The administration of a radiocolloid agent for SLN biopsy in breast cancer surgery has been described by numerous injection routes, including intraparenchymal (peritumoral), intradermal, subdermal, subareolar, and intratumoral [133]. Within the United States, the three predominant injection routes have been intraparenchymal, intradermal, and subareolar. Until recently, all data comparing these injection routes has been retrospective in nature. However, recently, the first prospective randomized clinical trial comparing the intraparenchymal, intradermal, and subareolar injection routes was conducted among 400 breast cancers at The Ohio State University and utilizing a dose of approximately $0.4 \mathrm{mCi}$ (14.8 MBq) of $99 \mathrm{mTc}$ sulfur colloid [141]. This recently 
reported study demonstrated superior intraoperative gamma probe localization of $99 \mathrm{~m}$ Tc sulfur colloid within the axillary lymph nodes for the intradermal route $(100 \%)$, as compared to the subareolar route $(95 \%)$ and intraparenchymal route (90\%) [141]. Additionally, this study demonstrated that intradermally injected 99mTc sulfur colloid resulted in the greatest maximum sustainable ex vivo counts within the hottest axillary SLN and the shortest intraoperative time to harvest the axillary SLN.

The determination of an adequate intraoperative assessment of the axilla with the gamma detection probe during breast cancer surgery is clearly related to the number of SLN candidates harvested and meticulous intraoperative attention to attempting to identify all potential sentinel lymph node candidates with counts of at least $10 \%$ of the counts of the hottest SLN. The most compelling argument for this is based on the results of two reports published by the University of Louisville Breast Cancer Sentinel Lymph Node Multiinstitutional Study Group [142,143] which evaluated patients undergoing SLN biopsy and a concomitant confirmatory axillary lymph node dissection. In 2001, Wong et al [142] demonstrated a false negative rate of $14.3 \%$ in patients who had a single SLN was harvested as compared to $4.3 \%$ in patients who had two or more SLNs were harvested $(\mathrm{P}=0.0004)$. Identically, in 2005, Martin et al [143] reproduced those same results, demonstrating a false negative rate of $13.7 \%$ in patients who had a single SLN harvested as compared to $5.4 \%$ in patients who had two or more SLNs harvested $(\mathrm{P}<0.0001)$. A reemphasis of this concept has been brought back to our attention in several more recently published reports [144147]. In 2007, Povoski et al [144] demonstrated that although $83 \%$ of cases had the first positive SLN identified as the hottest SLN, $17 \%$ of cases had the first positive SLN identified as the second, third, fourth, or fifth hottest SLN. Additionally, in this report, the SLN was positive in a significantly greater frequency of cases in which two or more SLNs were identified as compared to when only a single SLN was identified (34\% versus $18 \%, \mathrm{P}=0.003$ ). Likewise, Woznick et al [145] reported in 2006 that although $77 \%$ of cases had the first positive SLN identified as the first removed SLN, $23 \%$ of cases had the first positive SLN identified as the second, third, fourth, fifth, or sixth SLN removed. Similarly, Wada et al [146] reported in 2007 that although $76 \%$ of cases had the first positive SLN identified as the hottest SLN, 14\% of cases had the first positive SLN node identified as the second and third hottest removed SLN, with an additional 3\% having the positive SLN identified as a non-radioactive, blue-stained SLN, and an additional $7 \%$ having false-negative results. Finally, Krag et al [147], in the NSABP (National Surgical Adjuvant Breast and Bowel Project) B-32 randomized phase 3 trial, clearly demonstrated a significant association of the reduction in the observed false-negative rate as the total number of SLNs that were removed increased (i.e., with a $17.7 \%, 10.0 \%, 6.9 \%, 5.5 \%$, and $1.0 \%$ falsenegative rate when one, two, three, four, and five or more SLNs were removed, respectively). These six studies [142147], when taken together, raise significant concern with the scenario in which only a single negative SLN candidate is intraoperatively identified with the gamma probe and emphasizes the importance of a meticulous intraoperative search for additional SLN candidates with counts of at least $10 \%$ of the counts of the hottest SLN.

\section{RIGS}

The application of RIGS technology to the breast has been previously investigated and reported in a very limited fashion [148-152]. In 1989, Nieroda et al [148,149] intraoperatively evaluated 14 patients with breast cancer with the gamma detection probe after injection $5 \mathrm{mCi}$ (185 MBq) of ${ }^{125}$ I-labeled monoclonal antibody B72.3 at a time of six to 24 days prior to the surgical procedure. In 1996, and again identically re-reported in 1998, Percivale et al [150] and Badellino et al [151] intraoperatively evaluated 21 patients with locally advanced breast cancer with the gamma detection probe after injection of either 1.5 $\mathrm{mCi}(56 \mathrm{MBq})$ of ${ }^{125}$ I-labeled monoclonal antibody B72.3 or $1.5 \mathrm{mCi}(56 \mathrm{MBq})$ of ${ }^{125}$ I-labeled fragments of the murine anti-CEA monoclonal antibody F023C5 at a mean time of 21.7 days or 10.3 days, respectively, prior to the surgical procedure. In 2001, Burak et al [152] intraoperatively evaluated 10 patients with breast cancer with the gamma detection probe after injection $2 \mathrm{mCi}(74 \mathrm{MBq})$ of 125I-labeled NR-LU-10 antibody fragments at a time of two to seven days prior to the surgical procedure. In all three instances, specific binding to histologically confirmed sites of breast cancer was noted. However, the clinical impact of this technology in the context of these $125 \mathrm{I}-$ labeled-antibody complexes has not been more recently further investigated in the arena of breast cancer surgery.

\section{Radioguided occult lesion localization (ROLL)}

Radioguided occult lesion localization (ROLL), using gamma detection probe technology, is well described in the literature as an alternative to conventional wire localization for nonpalpable breast lesions, including breast cancer [153-181]. Most commonly, this ROLL technique involves an intratumoral injection (using preoperative same-day or prior-day mammographic or ultrasound guidance) of a 99mTc-based agent (such as colloidal human serum albumin, nanocolloid, macroaggregate albumin, or dextran) in a dosing range from $0.05 \mathrm{mCi}$ (1.8 MBq) to $4.0 \mathrm{mCi}(148 \mathrm{MBq})[153-155,157,158,160$ $171,173-181]$. As a result of the intratumoral injection of a 99mTc-based agent, this ROLL technique allows for simultaneous performance of a radioguided SLN biopsy procedure. In the largest retrospective series to date, Monti et al [168], 959 patients with cytologically or histologi- 
cally proven breast cancer underwent ROLL plus radioguided SLN biopsy, with successful breast lesion localization in $99.6 \%$ of cases and negative surgical margins obtained in $91.6 \%$ of cases. Additionally, a ROLL technique involving placement of a radioactive seed (consisting of a 4.5 $\mathrm{mm}$ by $0.8 \mathrm{~mm}$ titanium seed containing $0.125 \mathrm{mCi}$ ( 4.6 $\mathrm{MBq})$ to $0.29 \mathrm{mCi}(10.7 \mathrm{MBq})$ of $\left.{ }^{125} \mathrm{I}\right)$ up to five days in advanced to the date of surgery $[156,159]$ has been described. Finally, and most recently, ROLL techniques have been described utilizing an intravenous intraoperative injection of $20 \mathrm{mCi}(740 \mathrm{MBq})$ of 99mTc sestamibi [167] on the day of surgery and utilizing an intravenous injection of $6 \mathrm{mCi}(222 \mathrm{MBq})$ of ${ }^{111}$ In pentetreotide [172] at approximately 24 hours prior to surgery. In a systematic review detailing the available data in the literature on the ROLL technique, it was concluded that the ROLL technique compared favorably to that of conventional wire localization for nonpalpable breast lesions [176]. A largescale (over 300 patients), multicenter, prospective clinical trial in the Netherlands to evaluate the ROLL technique compared to conventional wire localization for nonpalpable breast cancers is currently being planned [182].

\section{Radioguided intraoperative margins evaluation (RIME)}

Radioguided intraoperative margins evaluation (RIME), using gamma detection probe technology, is limitedly described in the literature as a technique for not only guiding resection of the primary tumor but also for intraoperative assessment and determination of the adequacy of surgical margins $[183,184]$. The feasibility of this technique was first described utilizing a preoperative sameday intravenous injection of $0.172 \mathrm{mCi}(6.4 \mathrm{MBq})$ of $125 \mathrm{I}-$ labeled Lanreotide (a radiolabeled somatostatin analog) [183]. More recently, feasibility of the RIME technique was described utilizing a preoperative same-day intravenous injection of $20 \mathrm{mCi}(740 \mathrm{MBq})$ of $99 \mathrm{mTc}$ sestamibi [184].

\section{F-FDG-directed surgery}

Very recently, the application of gamma detection probe technology in radioguided surgery after a preoperative same-day injection of ${ }^{18}$ F-FDG has been reported in a limited number of selected cases of breast cancer $[43,45,47,51]$. In this regard, and of most recent note, our own group at The Ohio State University has recently reported a combined approach of perioperative ${ }^{18} \mathrm{~F}-\mathrm{FDG}$ PET/CT imaging (using a triad of preoperative PET/CT imaging, specimen PET/CT imaging, and postoperative PET/CT imaging) and intraoperative gamma detection probe technology, using a dose of approximately 14 to 19 mCi (518 to $703 \mathrm{MBq}$ ) ${ }^{18} \mathrm{~F}-\mathrm{FDG}$ injected intravenously approximately 120 minutes prior to the anticipated time of surgery for two selected cases of breast cancer for guiding tumor localization and for verifying complete tumor resection [51].

\section{Cutaneous malignancies}

Malignant melanoma

The single most important and most widely utilized application of gamma detection probe technology for the surgical management of malignant melanoma is radioguided SLN biopsy. More recently, the application of ${ }^{18}$ F-FDGdirected surgery to the management of appropriately selected cases of metastatic or recurrent malignant melanoma has received increasing interest within the surgical community.

\section{Radioguided SLN biopsy}

The application of SLN biopsy for the surgical staging of the regional lymph node basins during cutaneous malignant melanoma surgery has become widely accepted as a standard of care for cutaneous malignant melanoma of the trunk, extremities, and head and neck region [185]. To date, over 1,800 articles have been published which have been sited in PUBMED under the search descriptors of "melanoma" and "sentinel lymph node".

SLN biopsy in cutaneous malignant melanoma was first described in 1992 by Morton et al [186] using a technique of intradermally injected vital blue dye alone. In 1993, Uren et al [187] described a combined technique, consisting of preoperative lymphoscintigraphy (using 99mTc antimony sulfide colloid) for identification of all sites of location that are simply marked on the skin surface with an indelible marker and of intraoperative intradermally injected vital blue dye (but without use of an intraoperative gamma detection probe), for SLN detection. Shortly thereafter in 1993, the intraoperative use of the gamma detection probe for radioguided SLN biopsy in cutaneous malignant melanoma was first reported by Alex et al [188] at The University of Vermont in ten patients using intradermally injected $99 \mathrm{mTc}$ sulfur colloid (Table 1 ). Since that time, it has been clearly demonstrated that the use of radioguided SLN biopsy is superior to the vital blue dye alone technique for the surgical evaluation of at-risk nodal basins for cutaneous malignant melanoma [189-198]. The most compelling and well-respected international evidence for this comes from the Multicentric Selective Lymphadenectomy Trial Group results that were published in 1999 by Morton et al [193] on 570 melanoma patients. They demonstrated that the success of SLN identification was significantly improved by a combined radiocolloid and vital blue dye technique $(\mathrm{n}=217)$ as compared to a vital blue dye alone technique $(\mathrm{n}=352)$ (99.1\% versus $95.2 \%$, respectively; $\mathrm{p}=0.014$ ).

The primary $99 \mathrm{mTc}$-based agents utilized for radioguided SLN biopsy for cutaneous malignant melanoma are either $99 \mathrm{mTc}$ sulfur colloid or $99 \mathrm{mTc}$ colloidal human albumin (i.e., ${ }^{99 m}$ Tc nanocolloid) [199-206]. Generally, these radiocolloid agents are most commonly administered one to 
six hours prior to the planned surgical procedure in a dosing range from $0.4 \mathrm{mCi}(14.8 \mathrm{MBq})$ to $1 \mathrm{mCi}(37 \mathrm{MBq})$. Radiocolloid is exclusively injected intradermally and is administered in one to four sites around the intact primary lesion or around the resultant excisional biopsy scar.

It is clearly evident that preoperative lymphoscintigraphy plays as important of a role as does intraoperative radioguided SLN biopsy for the successful identification of all potential SLN candidates during the surgical management of cutaneous malignant melanoma secondary to the potential for localization to multiple nodal basins $[196,203,207-214]$ and for localization to in-transit (interval) SLNs [215-219]. For cutaneous malignant melanoma of the truncal region, preoperative lymphoscintigraphy localization to multiple nodal basins has been demonstrated in $17 \%$ to $32 \%$ of patient undergoing radioguided SLN biopsy [196,203,207-214]. Likewise, preoperative lymphoscintigraphy localization to in-transit (interval) SLNs has been demonstrated in 3\% to $10 \%$ of patient undergoing radioguided SLN biopsy for cutaneous malignant melanoma of the extremities and of the head and neck region [215-219]. Therefore, unlike other malignancies in which radioguided SLN biopsy can be performed without the antecedent need of preoperative lymphoscintigraphy, the radioguided surgical approach to cutaneous malignant melanoma should include both preoperative lymphoscintigraphy and intraoperative utilization of the gamma detection probe.

The determination of an adequate intraoperative assessment of any given nodal basin with the gamma detection probe during radioguided SLN biopsy for cutaneous malignant melanoma is clearly related to the meticulous intraoperative attention to attempting to identify all potential SLN candidates with counts of at least $10 \%$ of the counts of the hottest SLN [220-225]. The first compelling argument for the $10 \%$ rule for malignant melanoma came from work published by McMasters et al [221] from the Sunbelt Melanoma Trial in which they found that within $13.1 \%$ of positive lymph node basins that the most radioactive SLN was negative for tumor while a less radioactive sentinel lymph node was positive for tumor. Additionally, in $50 \%$ of such cases, they found that these less radioactive positive SLNs contained $50 \%$ or less of the radioactive counts of the hottest non-positive SLN. Similarly, Carlson et al [222] in 2002, Jacob et al [223] in 2003, and Kroon et al [224] in 2007 found in $19.1 \%$, $19.0 \%$, and $11.0 \%$ of all positive SLN cases, respectively, that the hottest SLN was negative for tumor. As a result, all these reports have strongly advocated applying the $10 \%$ rule to reduce the risk of a missed lymph node metastasis during radioguided SLN biopsy for cutaneous malignant melanoma [220-225].
In addition to the vast body of data in the literature on the application of radioguided SLN biopsy for the surgical staging of conventional sites of cutaneous malignant melanoma (i.e., trunk, extremities, and head and neck region), various case reports and small series reports exist on the potential application of radioguided SLN biopsy for the surgical staging of less conventional sites of malignant melanoma, such as the ocular conjunctiva and periocular skin [226-234], the vulvar region [235-240], the vagina [236,238,240-242], and the anal canal [243-248]. For all these less conventional sites of malignant melanoma, the feasibility of radioguided SLN biopsy has been demonstrated. However, larger scale evaluation of this technology for these less conventional sites of malignant melanoma is obviously necessary in order to better assess its clinical relevance.

\section{F-FDG-directed surgery}

The ${ }^{18}$ F-FDG avidity of malignant melanoma has made the application of ${ }^{18}$ F-FDG PET imaging paramount to the accurate staging and restaging of malignant melanoma [185]. Likewise, ${ }^{18}$ F-FDG PET imaging and ${ }^{18}$ F-FDG PET/ CT imaging have been shown to lead to a change in the clinical management of $17 \%$ to $39 \%$ of malignant melanoma patients, which is far greater than with other conventional imaging techniques alone [249-253]. While preoperative utilization of 18 F-FDG PET imaging has become a standard of care for the clinical management of selected cases of malignant melanoma, the intraoperative use of ${ }^{18} \mathrm{~F}-\mathrm{FDG}$ in combination with gamma detection probe technology has also been proposed and described in the clinical management of selected cases of metastatic malignant melanoma and has received increasing interest within the surgical community $[38,40,41,43,47,52]$.

The intraoperative use of $18 \mathrm{~F}-\mathrm{FDG}$ in combination with gamma detection probe technology for selected cases of metastatic malignant melanoma was first reported in 2001 by Essner et al [38]. Six patients were intravenously injected with 7 to $10 \mathrm{mCi}$ ( 259 to $370 \mathrm{MBq}$ ) of ${ }^{18} \mathrm{~F}-\mathrm{FDG}$ within three hours of the planned time of surgery. They reported that the tumor to background count ratio varied from 1.16:1 to 4.67:1, with eight of 13 melanoma metastases having a tumor-to-background count ratio of greater than 1.5:1. In 2005, Franc et al [41] reported on 5 patients with recurrent malignant melanoma that were injected with $14.6 \pm 3.2 \mathrm{mCi}(540 \pm 118 \mathrm{MBq})$ of 18 F-FDG. They found that the use of gamma detection probe technology had a sensitivity of $89 \%$ and a specificity of $100 \%$ for the detection of tissues containing recurrent malignant melanoma. More recently in 2006, Gulec et al [43] reported on 26 patients with either recurrent or metastatic malignant melanoma that were intravenously injected with 7 to $10 \mathrm{mCi}$ ( 259 to $370 \mathrm{MBq}$ ) of ${ }^{18}$ F-FDG within one to four hours of the planned time of surgery. They 
found that the tumor-to-background ratio ranged from $1.5: 1$ to $2.5: 1$, with a mean of $1.8: 1$, and metastatic lesions as small as $5 \mathrm{~mm}$ were detected.

The specific application of a combined approach of preoperative ${ }^{18} \mathrm{~F}-\mathrm{FDG}$ PET/CT imaging and intraoperative gamma detection probe technology for recurrent malignant melanoma was first described in 2005 by Carrera et al [40] in a patient intravenously injected with $6.8 \mathrm{mCi}$ (250 MBq) of ${ }^{18} \mathrm{~F}-\mathrm{FDG}$ at approximately four hours prior to the planned time of surgery, illustrating the potential advantage to a combination of these technologies. Furthermore, the added benefit to such combination technologies for ${ }^{18} \mathrm{~F}$-FDG-directed surgery was most recently illustrated by our own group at The Ohio State University in which a multimodality approach of perioperative ${ }^{18} \mathrm{~F}-$ FDG PET/CT imaging (preoperative patient imaging, specimen imaging, and postoperative patient imaging), intraoperative gamma detection probe technology, and intraoperative ultrasound were utilized for tumor localization and resection of all sites of hypermetabolic activity in a case of occult recurrent metastatic malignant melanoma [52]. In this case, the patient was intravenously injected with $12.8 \mathrm{mCi}(474 \mathrm{MBq})$ of ${ }^{18} \mathrm{~F}-\mathrm{FDG}$ at approximately 80 minutes prior to the planned time of surgery and this multimodality approach allowed for successful identification and resection of all three occult sites of recurrent metastatic malignant melanoma. Such refinements in multimodal ${ }^{18}$ F-FDG-directed surgery $[40,52]$ may allow this approach to further positively impact upon the established survival advantage of complete surgical resection for malignant melanoma patients with limited metastatic disease [254].

\section{Merkel cell carcinoma}

The application of the gamma detection probe in radioguided surgery for Merkel cell carcinoma has been limited to radioguided SLN biopsy. In this regard, SLN biopsy for the surgical staging of the regional lymph node basins is generally accepted as a standard of care for previously untreated, clinical stage I Merkel cell carcinoma $[255,256]$. The utilization of radioguided SLN biopsy for Merkel cell carcinoma was first reported in 1997 by Messina et al [257]. Since that time, multiple reports have been published on the application of radioguided SLN biopsy in the surgical management of Merkel cell carcinoma [258-274].

The technique of radioguided SLN biopsy for Merkel cell carcinoma is very similar to that for malignant melanoma. The primary $99 \mathrm{mTc}$-based agents utilized for radioguided SLN biopsy for Merkel cell carcinoma are either 99mTc sulfur colloid or and ${ }^{99 \mathrm{~m} T c}$ colloidal human albumin. These radiocolloid agents are generally administered on the morning of the planned surgical procedure in a dosing range from $0.3 \mathrm{mCi}(11 \mathrm{MBq})$ to $4 \mathrm{mCi}(150 \mathrm{MBq})$, are exclusively injected intradermally, and are administered in one to four sites around the intact primary lesion or around the resultant excisional biopsy scar [257-274].

The two largest radioguided SLN biopsy series for Merkel cell carcinoma reported to date were published by Allen et $\mathrm{al}$ in 2001 [264] and Maza et al in 2006 [272]. Allen et al [264] reported on 26 patients with clinical stage I Merkel cell carcinoma in which a SLN was detected in all patients using $99 \mathrm{mTc}$ sulfur colloid and metastatic disease was found in five (19\%) patients. Maza et al [272] reported on 23 patients with clinical stage I Merkel cell carcinoma in which a SLN was detected in all patients using ${ }^{99 \mathrm{mTC}} \mathrm{Tol}-$ loidal human albumin and metastatic disease was found in eleven (48\%) patients.

\section{Other cutaneous malignancies}

Radioguided SLN biopsy has been limitedly investigated in other cutaneous malignancies. It has been described for high risk squamous cell carcinoma of the facial region (face, ears, nose, and lips) $[268,269,275-278]$ and of the trunk and extremities [268,269,279-283], for high risk basal cell carcinoma [284,285], for sweat gland carcinoma [269,286-288], and for cutaneous lymphoma [269]. The technique described in the literature for radioguided SNL biopsy for these other cutaneous malignancies is identical to the technique described for malignant melanoma and Merkel cell carcinoma.

\section{Gastrointestinal malignancies \\ Colorectal cancer \\ RIGS overview}

The concept of RIGS in colorectal cancer [58] was first introduced in 1984 by the work of Aitken and colleagues $[289,290]$ at The Ohio State University (Table 1$)$. Using CEA-producing human colonic adenocarcinoma cells (CX-1) grown as tumor xenografts that were subcutaneously implanted on the flank in Swiss nude mice, they demonstrated the feasibility of gamma probe detection of ${ }^{131}$ I-labeled anti-CEA polyclonal baboon antibodies within such subcutaneous implants and demonstrated the greater sensitivity of the gamma detection probe as compared to gamma camera imaging for small tumor implants [289,290]. In addition to these experimental results in mice, they also reported the first clinical application of RIGS in a case study of a 59 year-old male with a rectal carcinoma [290]. The handheld gamma probe detected an increased level of the ${ }^{131}$ I-labeled anti-CEA polyclonal baboon antibody in the rectal tumor (135 counts/minute) compared to the sigmoid colon (111 counts/minute).

Shortly thereafter, Martin et al. [291] reported the results of the first RIGS clinical series involving 28 patients with 
primary $(n=12)$ and recurrent $(n=16)$ colorectal cancer. Each patient was injected intravenously with $2.2 \mathrm{mCi}$ (81.4 MBq) of ${ }^{131}$ I baboon polyclonal anti-CEA antibody at approximately 48 to 72 hours prior to surgery. Twentythree patients underwent preoperative scintigraphy. In all patients, intraoperative counts (20 seconds per count) of gross tumor and adjacent tissues were obtained using a prototype handheld gamma detection probe and a commercial control unit. Preoperative scintigraphy yielded correct results in $33 \%$ and $64 \%$ of the patients with primary and recurrent disease, respectively. In comparison, high intraoperative tumor-to-background ratios were achieved in all patients with primary tumors (3.91:1) and recurrent tumors (4.18:1). This clinical feasibility study demonstrated the ability of RIGS technology to provide immediate intraoperative information for the assessment of colorectal cancer. In addition, this study was an early indication of the application of the inverse square law and the advantage of a gamma detection probe [8]. The inverse square law states the sensitivity or specificity will increase as the distance from the detector is decreased. Hence, the intraoperative use of the gamma detection probe increased the probability of tumor detection due to its proximity to the source of radioactivity.

In all subsequent RIGS clinical studies, ${ }^{125}$ I was selected to replace ${ }^{131}$ I $[26,101,292] .{ }^{125}$ I was selected as the radionuclide of choice for RIGS since the handheld gamma detection probe was more efficient at detecting ${ }^{125}$ I than ${ }^{131} \mathrm{I}$ secondary to the lower energy level of the primary gamma photon emitter of ${ }^{125} \mathrm{I}(35 \mathrm{keV}$ ) as compared to the primary gamma photon emitter of ${ }^{131} \mathrm{I}$ (364 keV). Such lower energy gamma photons were more likely to be detected because the radiation was less likely to pass through a small detector without being counted. Likewise, in tumorbearing mice, higher tumor-to-background ratios were achievable with ${ }^{125}$ I labeled antibodies than with ${ }^{131}$ I labeled antibodies [293]. This resultant improved tumorto-background ratio was a function of greater tissue attenuation and lesser scatter that occurs because of the lower energy gamma photon emission of 125 I $[8,26]$. Likewise, the majority of clinical trials for RIGS in the detection of colorectal cancer have specifically utilized ${ }^{125}$ I-labeled anti-TAG-72 monoclonal antibodies. As previously discussed, such anti-TAG-72 monoclonal antibodies have evolved from first generation 125I-labeled murine B72.3 monoclonal antibodies, to second-generation 125 I-labeled murine CC49 monoclonal antibodies, and finally to third-generation 125I-labeled humanized CC49 (HuCC49) monoclonal antibodies [49].

\section{RIGS with I 7-IA murine monoclonal antibody}

The availability of the monoclonal antibody $17-1 \mathrm{~A}$ and its antibody fragment provided preliminary indication of the utility of 125 I and a gamma detection probe to discrimi- nate between tumor and background tissue and detect occult disease [101]. In 1986, O'Dwyer and colleagues [294] at The Ohio State University reported a 75\% intraoperative tumor localization rate among patients with primary and recurrent colorectal cancer. Higher tumor-tobackground ratios were observed in patients intravenously injected with the 125I-17-1A whole monoclonal antibody (3.4:1) compared to its radiolabeled monoclonal antibody fragment $\mathrm{F}\left(\mathrm{ab}^{\prime}\right)_{2}(2.3: 1)$. Of the 16 evaluable patients (two patients excluded due to a probe malfunction), histologically confirmed occult or subclinical disease was detected in $18 \%$ of these patients that would have otherwise not been clinically detected by normal intraoperative inspection and palpation. This early study was useful in shedding light on the optimal timing between injection and surgery for enhancing intraoperative gamma detection.

Petty and colleagues [295] at The Ohio State University continued the investigation of the 125I-17-1A monoclonal antibody in thirteen patients with colorectal cancer and optimized the use of the gamma detection probe to determine the clearance of the blood-pool background between the time of injection and surgery. The scheduling of the operative procedure was dependent on external precordial counts of $\leq 10$ per second obtained by the gamma detection probe. Intraoperative detection by the gamma detection probe was facilitated by the incorporation of a microprocessor into the control unit. The microprocessor maintained a running average of the count rate and made a synthetic siren sound when the count rate exceeds the background count by a statistically significant amount [8]. The squelching feature of the control unit allowed for a siren sound and no sound discrimination between normal tissues and areas of increased radioactivity without interfering with the count rates. Overall tumor localization rate of ${ }^{125}$ I-17-1A monoclonal antibody was $61 \%$ [295]. Localization was 33\% among primary tumor patients and $85 \%$ among recurrent tumor patients. Of the 12 tumor sites localized and biopsied, histologically confirmed tumor was identified in 8 (66\%) using hematoxylin and eosin (H\&E) staining. Additional studies using immunohistochemical staining and autoradiography identified malignant cells in all specimens. In comparison to the study reported by O'Dwyer et al [294], a higher occult disease detection rate was attributed to a lower blood-pool background at operation and improvements in the gamma detecting probe [295]. Predictability of ${ }^{125} \mathrm{I}$ 17-1A monoclonal antibody blood clearance was feasible using precordial counts. The majority of patients $(85 \%)$ demonstrated adequate clearance by 10 days post-injection of 125I-17-1A monoclonal antibody. Of the seven patients with recurrent disease, occult disease was identified in $43 \%$ of these patients. The finding of occult disease altered the surgical management (i.e., extended resection) 
of six patients. This study demonstrated the ability of the RIGS system to provide immediate intraoperative information regarding the extent and localization of disease by identifying tissues that did not look or feel abnormal but which were worthy of attention due to a siren signal/ sound generated by the gamma detection probe.

\section{RIGS with B72.3 murine monoclonal antibody}

The first generation of radiolabeled anti-TAG-72 monoclonal antibodies utilized the B72.3 murine monoclonal antibody. From preclinical studies on human colon carcinoma xenografts, significant tumor uptake of ${ }^{131}$ I-B72.3 monoclonal antibody was found to occur within 48 hours and subsequently increased over time [296]. Although blood-pool background clearance yielded higher tumorto-background ratios, the activity within the xenograft tumors remained constant over the 19 day period. This prolonged retention of the radiolabeled B72.3 monoclonal antibody and high degree of tumor targeting demonstrated in preclinical studies resulted in the selection of the B72.3 monoclonal antibody for subsequent use in RIGS [296,297].

Martin et al [298] reported the use of the ${ }^{125 I-B 72.3 ~ m o n-~}$ oclonal antibody and an improved gamma detection probe in human clinical trials initiated at The Ohio State University in 1986 [299] (Table 1). Of the 66 patients with gastrointestinal, breast, and ovarian carcinoma, six and 39 had primary and recurrent colon cancer, respectively [298]. Each patient was injected intravenously with 4 to $5 \mathrm{mCi}$ (148 to $185 \mathrm{MBq}$ ) of ${ }^{125}$ I-B72.3 monoclonal antibody at approximately 5 to 42 days prior to surgery. Adequate clearance of the blood-pool background yielded a high percentage of tumors identified using RIGS. The mean interval between injection and surgery of 19 days demonstrated long retention of B72.3 monoclonal antibody and adequate amount of the radionuclide for intraoperative gamma probe detection. Tumor localization of 125I-B72.3 monoclonal antibody was observed in $83 \%$ and $79 \%$ of the patients with primary and recurrent colorectal cancer, respectively. Increasing the size of the probe detector crystal and modifications in the crystal mounting improved the counting rate by a factor of approximately 2.5 .

Prolonged retention of the radiolabeled B72.3 monoclonal antibody and adequate clearance of the blood-pool background at the time of operation was further demonstrated in a study of primary colon cancer [300]. Thirty primary colon cancer patients were intravenously injected with 1 to $5 \mathrm{mCi}$ ( 37 to $185 \mathrm{MBq}$ ) of 125 I-B72.3 monoclonal antibody and then underwent surgery after adequate clearance of the blood-pool background was determined by precordial gamma detection probe counts (mean 22.3 days, range 8 to 34 days). The ${ }^{125}$ I-B72.3 mon- oclonal antibody localized in histologically confirmed tumor among 23 of the 30 patients (77\%). Of the 23 patients, 30\% had occult disease in the liver, lymph nodes, and/or invasion in adjacent tissues identified by RIGS. In $23 \%$ of these patients, the surgical plan was modified due to the finding of occult disease. RIGS provided adequate information (i.e., gamma detection probe counts comparable to background) regarding tumor margins and histologically confirmed benign lesions of the liver and ovaries. Therefore, the RIGS system provided a more accurate intraoperative staging of disease and immediate confirmation of tumor margins.

In 1991, Martin and Carey [301] reported the relationship between the survival of 86 patients with recurrent colorectal cancer undergoing a second-look procedure and use of the RIGS system. All patients received a preoperative intravenous injection of $2 \mathrm{mCi}$ (74 MBq) of ${ }^{125}$ I-B72.3 monoclonal antibody at approximately 24 days prior to surgery. The abdomen and pelvis were explored using traditional surgical techniques of inspection and palpation. Prior to the use of the gamma detection probe, sites of tumor were noted and the surgeon declared his surgical plan. The surgical field was reexplored using the gamma detection probe to identify areas of increased radioactivity compared to normal adjacent tissues and to determine whether the findings would alter the planned procedure. Fifty-three patients (62\%) were deemed resectable by traditional techniques. However, only 40 patients (47\%) were determined to be resectable by RIGS. Retroperitoneal disease was a common finding for RIGS nonresectability. Two-, three-, four-, and five-year survival data were reported for three classifications of patients: RIGS resectable $(\mathrm{n}=40)$, traditional nonresectable $(\mathrm{n}=33)$, and RIGS nonresectable $(\mathrm{n}=13)$. Overall survival rate for the RIGS resectable group was $83 \%$, versus $21 \%$ and $31 \%$ for the traditional nonresectable and RIGS nonresectable groups, respectively. Significant differences in survival were observed in the RIGS resectable versus traditional nonresectable, $\mathrm{p}<0.0001$; RIGS resectable versus RIGS nonresectable, $\mathrm{p}<0.0008$; and non-significant differences were noted in the traditional nonresectable versus RIGS nonresectable groups $(\mathrm{p}=0.24)$. A two- and five-year survival comparison is provided in Table 4 for RIGS using ${ }^{125} \mathrm{I}$ B72.3 monoclonal antibody.

The safety and efficacy of 125I-B72.3 monoclonal antibody to localize in tumor and detect occult disease was evaluated in a multicenter clinical trial of 104 patients with primary or recurrent colorectal cancer [302]. All patients received a preoperative an intravenous injection of $2 \mathrm{mCi}$ (74 MBq) of 125 I-B72.3 monoclonal antibody at approximately 24 days prior to surgery. Tumor localization occurred in $78 \%$ of the patients. A total of 30 occult tumor sites were identified in 26 patients. Of all histologically 
Table 4: Two-year and five-year survival for RIGS with ${ }^{125}$ I-B72.3 monoclonal antibody

\begin{tabular}{lcc}
\hline $\begin{array}{c}\text { PATIENT } \\
\text { GROUP }\end{array}$ & $\begin{array}{c}\text { SURVIVAL: 2-YEAR } \\
\text { (\% PATIENTS) }\end{array}$ & $\begin{array}{c}\text { SURVIVAL: 5-YEAR } \\
\text { (\% PATIENTS) }\end{array}$ \\
\hline RIGS resectable & $95 \%$ & $60 \%$ \\
\hline Traditional nonresectable & $36 \%$ & $0 \%$ \\
\hline RIGS nonresectable & $57 \%$ & $0 \%$ \\
\hline
\end{tabular}

confirmed tumor sites, $9.2 \%$ represented clinically occult sites identified only by the gamma detection probe. Location of occult disease in primary patients included retroperitoneum, pelvis, periportal, and liver. Of the 72 patients with recurrent disease, 37 were deemed unresectable. In $27 \%$ of these patients, data provided by the RIGS led to the decision to abandon an attempted resection. In addition, the operative resection was extended in the remaining 35 patients due to RIGS positive findings. One patient developed an acute hypersensitivity reaction to the skin test using unlabeled B72.3 monoclonal antibody. Although $40 \%$ of the patients injected with ${ }^{125}$ I-B72.3 monoclonal antibody developed human anti-murine antibodies, the occurrence did not impact upon tumor localization. Serum TAG-72 levels analysis demonstrated no relationship between the circulating antigen and localization of 125 I-B72.3 monoclonal antibody. The safety analysis of ${ }^{125}$ I-B72.3 monoclonal antibody did reveal statistically significant changes in body temperature, systolic blood pressure, and hemoglobin concentration; however, these changes were clinically insignificant. This multiinstitutional study confirmed a relatively low toxicity profile for ${ }^{125}$ I-B72.3 monoclonal antibody and its utility in identifying occult disease and impact on the surgical management of patients with primary and recurrent colorectal cancer.

Additional investigations into RIGS with ${ }^{125}$ I-B72.3 monoclonal antibody were reported in 1998 by the group at The University of Genoa in Italy $[303,304]$. They evaluated RIGS in both 16 asymptomatic patients with a history of previously treated colorectal cancer who has a rising CEA [303] and in 64 patients with recurrent or metastatic colorectal cancer [304]. In the group of 16 asymptomatic patients with a history of previously treated colorectal cancer who has a rising CEA, they found recurrent disease in only nine of 16 patients $(56.2 \%)$ by traditional surgical exploration alone, but in 14 of 16 patients $(87.5 \%)$ using a combined approach of traditional surgical exploration and RIGS, thus demonstrating that RIGS detected over-looked recurrent disease in five of 16 patients (31.3\%) [303]. In the group of 64 patients with recurrent or metastatic colorectal cancer, they nonran- domly compared ${ }^{125}$ I-B72.3 monoclonal antibody in 30 patients to ${ }^{125}$ I-F023C5 monoclonal antibody fragment that reacts with CEA in 34 patients [304]. They determined that the correct RIGS identification of tumor sites occurred in $80.4 \%$ of the 125 I-B72.3 monoclonal antibody group and in $92.6 \%$ of the 125 I-F023C5 monoclonal antibody fragment group, with additional occult sites of tumor identified by RIGS that would modify surgical strategy in $23.3 \%$ of the 125 I-B72.3 monoclonal antibody group and in only $8.8 \%$ of the ${ }^{125}$ I-F023C5 monoclonal antibody fragment group. They concluded that $125 \mathrm{I}-\mathrm{B} 72.3$ monoclonal antibody is the first choice for RIGS in recurrent or metastatic colorectal cancer.

The use of ${ }^{111}$ In-B72.3 murine monoclonal antibody $[107,305,306]$ in radioguided surgery for colorectal cancer has been limitedly investigated. Renda et al [305] investigated the detection of ${ }^{111}$ In-B72.3 monoclonal antibody by RIGS in 8 patients with colorectal cancer, reporting one false-positive and an overall sensitivity of $62.5 \%$. Muxi et al [306] investigated the detection of ${ }^{111}$ In-B72.3 monoclonal antibody by radioimmunoscintigraphy and RIGS in 28 patients with colorectal cancer (18 primary and 10 recurrent) and demonstrated an overall sensitivity of radioimmunoscintigraphy and RIGS of $71.4 \%$ and $82.1 \%$, respectively. More recently, Hladik et al [107] investigated the combined use of ${ }^{111} \mathrm{In}-\mathrm{B} 72.3$ monoclonal antibody and ${ }^{99} \mathrm{Tc}-\mathrm{anti}-\mathrm{CEA}$ monoclonal antibody fragment ( ${ }^{9} \mathrm{Tc}-\mathrm{IMMU}-4$ monoclonal antibody fragment) and compared the findings yielded by preoperative immunoscintigraphy, RIGS, and histology (H \& E and immunohistochemistry). Of the 121 patients, 106 and 15 had primary and recurrent colorectal cancer, respectively. A more accurate diagnosis was achieved using preoperative immunoscintigraphy and RIGS. Of the 55 RIGS-positive lymph node patients, 43 cases (78\%) were confirmed by histologically, including 9 cases in which RIGS-positivity was recognized by immunohistochemistry alone. Of 66 patients with RIGS-negative results, 62 cases (94\%) were confirmed by negative histology. The authors concluded a potential use of RIGS in the surgical management of primary colorectal patients includes better intraoperative assessment of the extent of disease and staging by identifying occult lymph node disease. Nevertheless, the major drawback to the use of ${ }^{111}$ InB72.3 monoclonal antibody in radioguided surgery for the detection of colorectal cancer has been the nonspecific accumulation of the ${ }^{111}$ In-B72.3 monoclonal antibody conjugate within the liver, thus making it difficult to identify liver metastases and limiting its usefulness to the identification of extrahepatic disease [307].

\section{RIGS with CC49 murine monoclonal antibody}

The second generation of radiolabeled anti-TAG-72 monoclonal antibodies utilized the CC49 murine monoclonal 
antibody. CC49 murine monoclonal antibody demonstrated a higher affinity constant $\left(\mathrm{K}_{\mathrm{a}} 16.18 \times 10^{9} \mathrm{~m}^{-1}\right)$ compared to B72.3 monoclonal antibody $\left(\mathrm{K}_{\mathrm{a}} 2.54 \times 10^{9}\right.$ $\mathrm{m}^{-1}$ ) [72]. In 1992, Arnold et al [74] at The Ohio State University evaluated the efficiency of 125I-CC49 monoclonal antibody and its impact on the RIGS system in a clinical trial of colorectal cancer patients, including 24 primary tumors and 30 recurrent tumors. All patients received a preoperative intravenous injection of $2 \mathrm{mCi}$ (74 $\mathrm{MBq}$ ) of ${ }^{125} \mathrm{I}-\mathrm{CC} 49$ monoclonal antibody with a varying monoclonal antibody dosage at approximately 14 to 21 days prior to surgery. Tumor localization of the ${ }^{125}$ I-CC49 monoclonal antibody occurred in $86 \%$ and $97 \%$ of the primary and recurrent tumors, respectively. In comparison to ${ }^{125}$ I-B72.3 monoclonal antibody, 125I-CC49 monoclonal antibody tumor localization was superior in targeting primary and recurrent colorectal tumors. The intraoperative findings from RIGS altered the planned surgical procedure in $50 \%$ and $47 \%$ of the primary and recurrent patients, respectively. Likewise, the intraoperative identification of occult disease by RIGS resulted in upstaging of cancer and abandonment of hepatic resections in three patients undergoing a second-look procedure. In this study, tissue specimens were classified by whether they were detected by RIGS and by the presence or absence of histologically confirmed carcinoma. Specimens were divided into tissue types I through IV, based on antibody localization and hematoxylin and eosin staining: type I, RIGS negative and histologically negative; type II, RIGS negative and histologically positive; type III, RIGS positive and histologically negative; and type IV, RIGS positive and histologically positive. Of interest to the authors were the type III lymph nodes. The intraoperative detection of type III lymph nodes by RIGS was a function of the presence of the TAG-72 within the extracellular environment. At surgery, all RIGS-positive tissue was considered malignant and excised whenever possible. Type III lymph nodes were detected in both primary cases $(n=40$ specimens) and recurrent cases ( $\mathrm{n}=16$ specimens). In this regard, Quinlan et al [308] reported a relationship between the presence of CC49 monoclonal antibody in the germinal centers of lymph nodes and early death among patients with colorectal cancer.

The role of RIGS as an intraoperative prognostic indicator of survival in patients with primary colorectal cancer was investigated by Arnold et al [309]. Patients were intravenously injected with $2 \mathrm{mCi}$ (74 MBq) of ${ }^{125}$ I-CC49 monoclonal antibody at approximately 21 days prior to surgery. Thirty-one primary colorectal cancer patients with 125 I-CC49 monoclonal antibody localization were assessed for the presence or absence of residual RIGS-positive tissue at the completion of the operative procedure. Patients were classified as RIGS-positive (i.e., residual RIGS-positive tissue) or RIGS-negative (i.e., no residual
RIGS-positive tissue). Tumor localization was observed in $86 \%$ of the patients. One-hundred and nine extra-regional sites of RIGS-positivity were identified using RIGS, including but not limited to the gastrohepatic ligament, celiac axis, retroperitoneum, liver, omentum, and sites above the diaphragm. Seventeen and 14 patients were assessed as RIGS-positive and RIGS-negative, respectively, following the completion of the operative procedure. Follow up of patients ranged from 30 to 54 months. Of the 17 patients with residual RIGS-positive tissue, 15 (88\%) succumbed to their disease. In comparison, all 14 patients with no residual RIGS-positive tissue were alive at last follow-up ( 24 to 48 months after surgery) ( $<<0.0001$ ). Therefore, the presence or absence of residual RIGS-positive tissue provided immediate and accurate prognostic information regarding the behavior of the tumor and patient outcomes in patients with primary colorectal cancer.

Likewise, in the recurrent colorectal cancer population, Bertsch et al [310] reported an improved survival relative to the use of the RIGS system. One hundred and thirtyone patients with recurrent colorectal cancer were injected with ${ }^{125}$ I-B72.3 monoclonal antibody $(\mathrm{n}=86)$ or ${ }^{125} \mathrm{I}$ CC49 monocloncal antibody $(\mathrm{n}=45)$ and underwent a surgical exploration using traditional inspection and palpation and the RIGS system. Of the 49 patients deemed resectable (i.e., successful removal of all traditionally evident disease and all RIGS-positive tissue), 55\% (27 patients) were alive at 2 to 8 years following surgery, with a minimal follow-up of 28 months. In contrast, only $2.4 \%$ of all patients ( 2 of 84 patients) with unresectable disease were alive at the time of this report. None of the patients determined to be traditionally resectable but unresectable by the RIGS system were alive. A significant increase in survival $(\mathrm{p}<0.0001)$ was observed among patients with recurrent colorectal cancer undergoing a resection of all disease found by a combination of both traditional means and by RIGS.

A long-term survival analysis of 97 patients with primary colorectal cancer supports the use of RIGS as an intraoperative prognostic tool [311]. Survival was assessed using the traditional staging (TNM) and the presence or absence of RIGS-positive tissue at the end of the operative procedure. The mean follow-up among the 52 evaluable patients was 62 months (range 34 to 89 months). Based on TNM staging 13,18 , and 28 patients were Stage, I, Stage II, and Stage III, respectively. By RIGS status, 24 and 35 were RIGS-negative and RIGS-positive, respectively. The RIGS status was not significantly related to the traditional pathologic staging $(\mathrm{p}=0.73)$. No significant difference in survival was observed using standard pathologic staging $(p=0.12)$. However, a significant difference in survival was observed by the using the RIGS status $(\mathrm{p}<$ 
0.0002 ), with $87 \%$ of RIGS-negative patients alive and only $40 \%$ of RIGS-positive patients alive at a mean followup of 62 months.

The extended survival (i.e., 10-year survival) of 90 colorectal cancer patients undergoing RIGS, based on the presence or absence of residual RIGS-tissue at the time of surgery, was recently analyzed by Sun et al [49]. At 10 years after RIGS, survival differences remained significant ( $\mathrm{p}=0.001$ ), with $49 \%$ of the RIGS-negative patients (median survival of 106.5 months) still alive as compared to only $21 \%$ of the RIGS-positive patients (median survival of 26 months) still alive. These extended follow-up data support the accuracy of utilizing the RIGS status at the time of surgery as a predictor of long-term survival in colorectal cancer patients.

Additional investigations into RIGS with 125I-CC49 murine monoclonal antibody were reported in 2000 and 2001 by the group at Tel-Aviv University in Israel [312314]. Overall, they evaluated a total of 58 patients with recurrent colorectal cancer who were intravenously injected with of $2 \mathrm{mCi}(74 \mathrm{MBq})$ of ${ }^{125} \mathrm{I}-\mathrm{B} 72.3$ monoclonal antibody at approximately 24 days prior to surgery. Traditional surgical exploration and RIGS were performed. While traditional surgical exploration identified 117 suspected tumor sites, RIGS identified 177 suspected tumor sites. In 17 of 58 patients $(29.3 \%)$, at least one occult tumor site that was not identified by traditional surgical exploration was identified by RIGS, and was subsequently confirmed by pathology with $\mathrm{H} \& \mathrm{E}$ staining. This resulted in a major change in the surgical plan in 16 cases. RIGS performance was found to vary between lymphoid tissue and non-lymphoid tissue, with a positive predictive value and a negative predictive value of $96 \%$ and $90 \%$ in non-lymphoid tissue and $40 \%$ and $100 \%$ in lymphoid tissue, respectively.

\section{RIGS with humanized CC49 monoclonal antibody}

A phase I study of ${ }^{125} \mathrm{I}-\mathrm{HuCC} 49 \Delta \mathrm{C}_{\mathrm{H}} 2$ monoclonal antibody conducted at The Ohio State University determined the feasibility of the humanized monoclonal antibody as a component of the RIGS system and evaluated the optimal timing interval between injection and surgery [315]. Study eligibility included patients with recurrent colorectal cancer undergoing a surgical exploration and excluded those with prior exposure to murine antibodies. Patients underwent a surgical exploration at variable time intervals following the intravenous administration of $2 \mathrm{mCi}$ (74 $\mathrm{MBq})$ of ${ }^{125} \mathrm{I}-\mathrm{HuCC} 49 \Delta \mathrm{C}_{\mathrm{H}} 2$ monoclonal antibody or after the precordial counts measured by a handheld gamma detecting probe were less than 30 counts per two seconds. HAMA determinations were obtained at baseline, as well as at 4 to 6 weeks and 12 weeks post-injection of the ${ }^{125} \mathrm{I}-\mathrm{HuCC} 49 \Delta \mathrm{C}_{\mathrm{H}} 2$ monoclonal antibody. At sur- gery, traditional exploration (i.e., inspection and palpation) and subsequent RIGS exploration were performed. Suspicious tissues were biopsied or excised to determine the presence of absence of carcinoma. The findings were analyzed to determine the sensitivity and positive predictive value for each modality. Of the twenty recurrent colorectal cancer patients evaluated, the first 15 operative procedures were performed at various time intervals $(3,5$, $7,9,11$, and 13 days) after the injection of $125 \mathrm{I}$ HuCC $49 \Delta \mathrm{C}_{\mathrm{H}} 2$ monoclonal antibody and independent of the precordial counts. In the five remaining patients, the timing of surgery was determined by the demonstration of precordial counts of less than 30 counts per two seconds, indicating optimal clearance of ${ }^{125} \mathrm{I}-\mathrm{HuCC} 49 \Delta \mathrm{C}_{\mathrm{H}} 2$ monoclonal antibody from the blood-pool background. This occurred at 10 to 24 days after the injection of $125 \mathrm{I}$ HuCC $49 \Delta \mathrm{C}_{\mathrm{H}} 2$ monoclonal antibody. The adequate clearance of the blood-pool background in these five patients allowed for intraoperative differentiation of RIGS-positive tissue compared to normal adjacent tissue. Among those five patients with optimized clearance conditions based on precordial counts of less than 30 counts per two seconds, 17 and 21 sites were identified using traditional techniques (i.e., inspection and palpation) and RIGS system, respectively. Approximately 90\% of the excised tissues identified by traditional techniques and by RIGS were histologically positive for tumor. Of the six sites identified exclusively by RIGS, five were excised and all contained tumor. Table 5 provides the sensitivity of traditional exploration and RIGS exploration using ${ }^{125} \mathrm{I}-$ HuCC $49 \Delta \mathrm{C}_{\mathrm{H}} 2$ monoclonal antibody for identifying recurrent colorectal cancer and the positive-predictive value. No significant HAMA response was detected in these patients. Despite the small number of evaluable patients, the study demonstrated the safety and utility of ${ }^{125} \mathrm{I}-\mathrm{HuCC} 49 \Delta \mathrm{C}_{\mathrm{H}} 2$ monoclonal antibody in RIGS. The rate of tumor localization and detection of occult disease was comparable to murine CC49 previously reported by Arnold et al [74], but without a detectable HAMA response. The use of HuCC $49 \Delta \mathrm{C}_{\mathrm{H}} 2$ monoclonal antibody as a component of RIGS technology suggests that it is worthy of continued investigations in colorectal cancer patients.

Table 5: Traditional exploration and RIGS exploration with 125IHuCC $49 \Delta C_{\mathrm{H}} 2$ monoclonal antibody

\begin{tabular}{lcc}
\hline & Traditional exploration & $\begin{array}{c}\text { RIGS } \\
\text { exploration }\end{array}$ \\
\hline Sensitivity & $64 \%$ & $92 \%$ \\
\hline Positive predictive value & $90 \%$ & $100 \%$ \\
\hline
\end{tabular}


RIGS with ${ }^{125}$ I-anti-CEA monoclonal antibody $\left({ }^{125} /-A_{5} B_{7}\right)$ and ${ }^{99} T C-$ anti-CEA monoclonal antibody fragment (99TC-IMMU-4)

Limited information is available on use radiolabeled antiCEA monoclonal antibody in RIGS for colorectal cancer $[90,91,107,316]$. Dawson et al [91] evaluated 43 patients undergoing surgery with primary colorectal cancer and 9 patients undergoing second look laparotomy for recurrent colorectal cancer who were intravenously injected with 2 mCi (74 MBq) of ${ }^{125}$ I-anti-CEA monoclonal antibody $\left({ }^{125} \mathrm{I}-\mathrm{A}_{5} \mathrm{~B}_{7}\right)$ three to ten days before surgery. They determined that RIGS assessment of ${ }^{125} \mathrm{I}_{-} \mathrm{A}_{5} \mathrm{~B}_{7}$ localized in $97.8 \%$ of primary tumors and in $88.8 \%$ of most principle tumor sites found at the time of the second look procedure. Likewise, they determined that the use of RIGS influenced the overall surgical procedure performed in 2 of 43 cases $(4.6 \%)$ of primary colorectal cancer and in three of nine cases $(33 \%)$ of recurrent colorectal cancer. Lechner et al [316] evaluated 20 patients with primary colorectal cancer who were intravenously injected with $25 \mathrm{mCi}$ (925 $\mathrm{MBq})$ of ${ }^{9} \mathrm{Tc}$-anti-CEA monoclonal antibody fragment ( ${ }^{9}$ Tc-IMMU-4) on the day before RIGS. They determined that RIGS assessment of ${ }^{9} \mathrm{Tc}-\mathrm{IMMU}-4$ led to up-staging of disease in 7 of 20 patients (35\%). Hladik et al [107] evaluated 65 patients with either primary or recurrent colorectal cancer who were intravenously injected with 99TcIMMU-4 in a dose range of 18.9 to $24.3 \mathrm{mCi}$ (700 to 900 $\mathrm{MBq})$ on the day before RIGS. They determined that RIGS assessment of ${ }^{9} \mathrm{Tc}$ TcIMMU-4 led to a sensitivity and accuracy for the detection of tumor or local recurrence of $92 \%$ and $92 \%$, for the detection of hepatic metastases of $75 \%$ and $92 \%$, and for the detection of extrahepatic metastases of $57 \%$ and $78 \%$, respectively. Finally, Gu et al [90] evaluated 29 patients with primary colorectal cancer who were injected with $0.5 \mathrm{mCi}(18.5 \mathrm{MBq})$ of ${ }^{125}$ I-anti-CEA monoclonal antibody (125I-CL58) into the colonic submucosa at the tumor-surrounding areas (at 2, 4, 6, 8,10, and 12 o'clock locations) via colonoscopy at a time of approximately three to 14 days before RIGS. The sensitivity of RIGS in detecting primary lesions was $93.1 \%$, and the specificity of RIGS to correctly identify negative surgical margins was $95.5 \%$. For detection of lymph node metastases, the sensitivity and specificity of RIGS was $92.0 \%$ and $87.8 \%$, respectively.

\section{Radioguided SLN biopsy}

The application of SLN biopsy technology to colorectal cancer has been thoroughly studied. While the vast majority of the information available for SLN biopsy for colorectal cancer is on the application of the blue dye alone technique, the radioguided application of 99mTc sulfur colloid technique with intraoperative gamma probe detection has been limitedly investigated [317-324]. The results of the two largest reported series are strikingly divergent [319,323]. In 2003, Bilchik et al [319] evaluated 120 patients using a combined blue dye and 99mTc sulfur colloid (dose not reported) technique in 32 such patients. They reported successfully identifying a SLN in 115 of 120 $(96 \%)$ patients, with a false-negative SLN biopsy results identified in only 5 of 115 (4\%) patients. In contrast, in 2007, Lim et al [323] evaluated 120 patients using a combined blue dye and $99 \mathrm{mTc}$ sulfur colloid $(0.5 \mathrm{mCi} ; 19$ $\mathrm{MBq}$ ) technique in all patients. They reported successfully identifying a SLN in 119 of 120 (99\%) patients, with a falsely negative SLN biopsy results identified in 20 of 119 $(17 \%)$ patients. As a consequence of these strikingly different results with regards to the false-negative rate, we can realistically say that at the current time we are no closer to elucidating the clinical relevance of SLN biopsy technology for colorectal cancer surgery.

\section{F-FDG-directed surgery}

The most recent application of the gamma detection probe for radioguided surgery in colorectal cancer has been directed towards to identification of ${ }^{18} \mathrm{~F}-\mathrm{FDG}$-avid tumors [35-39,45,50]. This technique was first described by Desai et al $[35,36]$ at The Ohio State University (Table 1). Fourteen colorectal cancer patients received an intravenous injection of 4.0 to $5.7 \mathrm{mCi}$ ( 148 to $211 \mathrm{MBq}$ ) of ${ }^{18} \mathrm{~F}$ FDG at a time of 58 to 110 minutes prior to intraoperative evaluation with the gamma detection probe. Single or multiple tumor foci were correctly identified in 13 of 14 patients with the gamma detection probe as ${ }^{18}$ F-FDG-avid tissue and this correlated to hypermetabolic activity on prior preoperative diagnostic ${ }^{18}$ F-FDG PET imaging. These results have been further corroborated since that time in several other clinic reports [37-39,45,50]. Most recently, a combined approach of preoperative diagnostic ${ }^{18} \mathrm{~F}-\mathrm{FDG}$ PET imaging and intraoperative gamma probe detection has been advocated to potentially provide the surgeon with a real-time, intraoperative roadmap for accurately locating and determining the extent of tumor recurrence in patients with colorectal cancer [50]. In this series, patients received an average dose of 10 to $15 \mathrm{mCi}$ (370 to $555 \mathrm{MBq}$ ) of ${ }^{18}$ F-FDG at a time of 30 to 60 minutes prior to intraoperative evaluation with the gamma detection probe. It was determined that intraoperative evaluation with the gamma detection probe appeared to be more sensitive in detecting the extent of abdominal and pelvic recurrence, while preoperative ${ }^{18} \mathrm{~F}-\mathrm{FDG}$ PET imaging was more sensitive in detecting liver metastases and other distant metastases [50].

\section{Anal cancer}

The application of the gamma detection probe in radioguided surgery for anal cancer has been limited to radioguided SLN biopsy [324-337]. In general, 99mTc sulfur colloid, 99mTc colloidal human albumin, 99mTc colloidal rhenium sulphide, and $99 \mathrm{~m}$ Tc antimony trisulfide colloid have been utilized and are injected into four subdermal or submucosal sites around the primary tumor in a total dos- 
age range from $0.135 \mathrm{mCi}(5 \mathrm{MBq})$ to $1.0 \mathrm{mCi}(37 \mathrm{MBq})$ Localization to a SNL was generally demonstrated in $75 \%$ to $100 \%$ of cases. There were three common pathways of lymphatic drainage (including drainage to inguinal, iliac, and mesorectal lymphatic basins) and the very frequent finding of bilaterality of such lymphatic drainage, emphasizing the importance of preoperative lymphoscintigraphy in most effectively performing radioguided SLN biopsy in anal cancer. The inguinal region was the predominant lymphatic drainage pathway, representing the site of localization most accessible to performance of a minimally invasive radioguided SLN biopsy procedure. Radioguided SLN biopsy to the inguinal region was useful in identifying inguinal lymph node metastases in approximately $10 \%$ to $40 \%$ of such patients. However, there is yet to be a large scale prospective clinical trial to assess the clinical efficacy of radioguided SLN biopsy in anal cancer.

\section{Esophageal cancer}

The application of the gamma detection probe in radioguided surgery for esophageal cancer has been limited to radioguided SLN. In this regard, it is interesting to note that there have been as many review papers written that have debated the pros and cons of the potential clinic efficacy of radioguided SLN biopsy for esophageal cancer [324,338-343] as there have been actual papers written on the clinical results of this technology in esophageal cancer surgery. Clinical data on radioguided SLN biopsy for esophageal cancer are restricted to those reports from Japan [344-350], the United Kingdom [351], and Germany [352]. Central to the debate with regards to the potential clinical efficacy of radioguided SLN biopsy for esophageal cancer are the complex and extensive lymphatic networks located throughout cervical, mediastinal, and abdominal nodal basins and the concern for skip metastases [324,338,339,341-343]. In this regard, the routine use of radioguided SLN biopsy for esophageal cancer surgery remains controversial and is yet to be widely adopted.

Most frequently, $99 \mathrm{mTc}$ tin colloid has been used as the radiocolloid, especially in Japan [344-350]. However, 99mTc colloidal human albumin [351], 99mTc sulfur colloid [352], and $99 \mathrm{mTc}$ colloidal rhenium sulfide [347] have also been utilized. The dosing of these radiocolloid agents vary from as low as $0.54 \mathrm{mCi}(20 \mathrm{MBq})$ [351] to as high as $5.0 \mathrm{mCi}(185 \mathrm{MBq})[346,350]$. These radiocolloid agents are injected endoscopically in up to four submucosal sites around the tumor on either the day before surgery [344-350] or on the day of surgery [351,352].

The two largest series reported are by Lamb et al [351] and Kato et al [347]. In 2005, Lamb et al [351] identified a SLN in all 40 patients evaluated with adenocarcinoma of the lower esophagus. They identified a total of 77 SLNs from a combination of both the mediastinal and abdominal nodal stations, with $51 \%$ of these SLNs being located within the mediastinal nodal stations and with $38 \%$ of proven metastatic lymph nodes residing within the mediastinal nodal stations. Noteworthy from this study was the fact that if patients did not have a mediastinal SLN identified at the time of radioguided SLN biopsy that a negative abdominal SLN accurately predicted the absence of mediastinal lymph node involvement. Such a predictive property of SLN biopsy may help to alter the surgical strategy by possibly omitting a cervical nodal dissection for mid-esophageal tumors or by making the option of transhiatal esophagectomy more appropriate when mediastinal nodal clearance is not indicated. In 2003, Kato et al [347] identified a SLN in 23 of 25 (92\%) patients evaluated with squamous cell carcinoma of the thoracic esophagus. The accuracy of radioguided SLN biopsy was $91.3 \%$ (21 of 23 patients), the sensitivity was $86.7 \%$ (13 of 15 patients), and the false-negative rate was $8.7 \%$ (2 of 23 patients). Despite the promising clinical results seen with radioguided SLN biopsy for esophageal cancer, technical difficulties can be encountered with regards to identifying SLNs within the peritumoral nodal stations, particularly the lower paraesophageal, paracardial, and left gastric nodes, which are common sites of lymphatic metastases for lower esophageal cancers. As with other malignancies, the proximity of the SLN to the radiocolloid injection site creates an artifact shine through effect which can make identification of such located SLN with the gamma detection probe very difficult.

\section{Gastric cancer}

Although radioguided surgery is not routinely utilized in the current surgical management of gastric cancer, the application of the gamma detection probe for gastric cancer surgery has been investigated in the areas of radioguided SLN biopsy, RIGS, and ${ }^{18}$ F-FDG-directed surgery.

\section{Radioguided SLN biopsy}

The necessary extent of lymphadenectomy during gastric cancer surgery remains a controversial issue, with two randomized trials demonstrating no survival advantage for patients treated with D2 lymphadenectomy as compared to a D1 lymphadenectomy [353,354] compared with other retrospective reviews showing a survival benefit for patients treated with D2 lymphadenectomy [355,356]. In this regard, the potential application of SLN biopsy for gastric cancer remains an active avenue of clinical research and debate amongst surgeons who treat gastric cancer. This is most relevant for those clinicians in eastern Asia that primarily treat early stage gastric cancers, which by definition should have a less than $10 \%$ chance of nodal involvement [357]. 
Multiple reports exist in the literature on the feasibility of radioguided SLN biopsy for gastric cancer [338,344,345,358-383]. Many of these reports from eastern Asia specifically target a purely laparoscopic approach to radioguided SLN biopsy in the treatment of cases of early stage gastric cancer $[338,361,367,371,377,378,380$ 382].

Most frequently, ${ }^{99 \mathrm{~m} T \mathrm{Tc}}$ tin colloid [338,344-346,359$361,363-367,369,371,375-378,380,382]$ has been used as the radiocolloid, especially in eastern Asia. However, 99mTc colloidal rhenium sulfide $[362,368,374,379]$, $99 \mathrm{mTc}$ sulfur colloid [352,358,370,372], and $99 \mathrm{~m}$ Tc colloidal human albumin [381] have also been utilized. The dosing of these radiocolloid agents vary from as low as $0.5 \mathrm{mCi}$ (18.5 MBq) [374] to as high as $6.0 \mathrm{mCi}(222 \mathrm{MBq})$ [364,369]. These radiocolloid agents are generally injected endoscopically in up to four submucosal sites around the tumor at a time period from two to 24 hours before surgery.

The two largest series reported are by Kitagawa et al [359] and Uenosono et al [369]. In 2002, Kitagawa et al [359] identified a SLN in 138 of 145 patients (95.2\%) with presumed CT1N0 or CT2N0 gastric cancer. A SLN was positive in 22 of 24 patients who had lymph node metastases and this demonstrated a diagnostic accuracy of assessment of the regional lymph node status on the basis of the SLN status of $98.6 \%$. In 2005, Uenosono et al [369] identified a SLN in 99 of 104 patients (95.2\%) with presumed CT1 or CT2 gastric cancer. Excluding three technical failures in radiocolloid injection, identification rates were 99\% (78 of 79 ) and 95\% (21 of 22) for cT1 and cT2 lesions, respectively. Lymph node metastases and/or micrometastases were found in 28 patients ( 15 cT1 and 13 cT2) and the resultant false-negative rate, sensitivity, and accuracy were significantly better for CT1 tumors than for CT2 tumors (P $<0.001, \mathrm{P}=0.004$, and $\mathrm{P}<0.001$, respectively). While the possibility exists for radioguided SLN biopsy to help individualize the surgical therapy for patients with early stage gastric cancer, additional studies are needed to determine its utility.

\section{RIGS}

The feasibility of RIGS for gastric cancer has been evaluated by several groups of investigators [149,383-387]. In 1988, Martin et al [149] evaluated 125I-B72.3 murine monoclonal antibody in five patients with gastric cancer, finding positive gamma detection probe counts in four patients. In 1994, Xu et al [383] and Lui et al [384] evaluated ${ }^{131} \mathrm{I}-3 \mathrm{H} 11$ murine monoclonal antibody, a mouse antibody raised against human gastric cancer cells, in 25 patients with gastric cancer. They reported detection of metastatic lymph nodes with a sensitivity of $99.2 \%$, a specificity of $97.7 \%$, and an accuracy of $98.8 \%$, and reported the detection of tumor infiltration of the gastric wall with a sensitivity of $94.6 \%$, a specificity of $96.7 \%$, and an accuracy of 95.9\%. In 1998, Lucisano et al [385] evaluated 125I-B72.3 murine monoclonal antibody in 7 patients with gastric cancer. The correct RIGS identification of the primary tumor was seen in four of seven patients $(57.1 \%)$ and of metastatic lymph nodes in two of four patients (50\%). Also, in 1998, Mussa et al [386] evaluated ${ }^{111}$ InB72.3 murine monoclonal antibody in 3 patients with gastric cancer, confirming intraoperative tumor-to-background counts of greater than 2 in all three cases and demonstrating a sensitivity of $100 \%$, a specificity of $72 \%$, and no false-negative findings with RIGS. Finally, in 2000, Wang et al [387] evaluated ${ }^{125}$ I-3H11 murine monoclonal antibody in 35 patients with gastric cancer. For the detection of lymphatic metastases, they demonstrated a sensitivity of $83.6 \%$, a specificity of $95.0 \%$, and an accuracy of $91.3 \%$. The existence of lymphatic micrometastatic disease was verified immunohistochemically in 10 of 19 patients $(52.6 \%)$ that were RIGS-positive but that had H\&E histologically negative lymph nodes. Despite these early promising results, no further investigations into RIGS for gastric cancer have been published since that last report.

\section{${ }^{18} \mathrm{~F}$-FDG-directed surgery}

The use of ${ }^{18}$ F-FDG-directed surgery has been very limitedly reported in the literature for the surgical management of gastric cancer [45,47]. Gulec et al [45] reported on a single case of gastric cancer in which ${ }^{18} \mathrm{~F}$-FDG-directed surgery was utilized for the identification and resection of a hypermetabolic metastatic lymph node during a gastectomy and extended node dissection. Piert et al [47] reported on the use of ${ }^{18} \mathrm{~F}$-FDG-directed surgery in three gastric adenocarcinomas and two adenocarcinomas of the gastroesophageal junction.

\section{Pancreatic cancer}

Radioguided surgery using the gamma detection probe has surprisingly been previously investigated in only the most limited fashion for pancreatic cancer [388]. No data are currently available on radioguided sentinel lymph node biopsy or on ${ }^{18} \mathrm{~F}$-FDG-directed surgery for pancreatic adenocarcinoma. Only a single report exists in the literature from 1997 by LaValle et al [388] from The Ohio State University on RIGS for the assessment of extent of disease in ten cases of pancreatic adenocarcinoma that were deemed resectable by preoperative CT scan. Each patient was intravenously injected with $2 \mathrm{mCi}(74 \mathrm{MBq})$ of ${ }^{125} \mathrm{I}$ CC-49 murine monoclonal antibody and then underwent surgery after adequate clearance of the blood-pool background was determined by precordial gamma detection probe counts (mean 26.1 days, range 7 to 35 days). Traditional assessment of the abdomen was compared to RIGS assessment of the abdomen. Three patients underwent 
pancreatic resection for locoregional disease. The other seven patients had visceral metastases, carcinomatosis, or both detected at the time of laparotomy. All sites suspicious for tumor by traditional assessment of the abdomen were found to be RIGS positive. Occult pancreatic adenocarcinoma identified only by RIGS assessment was found to be disseminated to both the abdominal viscera and lymphatics. RIGS detected significantly more total sites (viscera and lymphatics) of metastatic disease than traditional assessment ( 73 site versus 31 sites for RIGS versus traditional assessment, respectively, $\mathrm{p}<0.05$ ), with the greatest difference being observed for dissemination to the lymphatics ( 44 site versus 6 sites for RIGS versus traditional assessment, respectively, $\mathrm{p}<0.001$ ). Despite these very encouraging early results, no further work has been done on RIGS for pancreatic cancer.

\section{Gastointestinal stromal tumors (GIST)}

The use of the gamma detection probe in radioguided surgery for GIST is virtually nonexistent. Only one report exists in the literature that describes two GIST cases in which 18F-FDG-directed surgery was employed [45]. In this report, ${ }^{18}$ F-FDG-directed surgery was used during exploratory cytoreduction and during liver resection for GIST.

\section{Head and neck malignancies \\ Squamous cell cancer of the oral cavity, oropharynx, hypopharynx, and laryngeal regions \\ Radioguided SLN biopsy}

While SLN biopsy had become a widely accepted diagnostic technique in the evaluation of the lymph node status in breast cancer and melanoma, it has been met with much more limited enthusiasm for squamous cell cancers of the head and neck region, including the oral cavity, oropharynx, hypopharynx, and laryngeal region, secondary to the complicated lymphatic drainage pathways of the head and neck region [389]. In 1996, Alex and Krag [390] first described successful radiolocalization of SLNs with ${ }^{99 \mathrm{~m} T c}$ sulfur colloid in the aerodigestive system in a patient with supraglottic squamous cell cancer. Since the time of this initial report, the sensitivity and specificity of radioguided SLN biopsy has become greater than that of physical exam, computed tomography, magnetic resonance imaging, or positron emission tomography for assessing the NO neck [391]. Additionally, the overall feasibility of radioguided SLN biopsy has gradually improved, with most recently reported success of localization of $99.3 \%$ in 137 patients by American College Of Surgeons Oncology Group Z0360 [392] and 100\% in 79 patients by Stoeckli [393] for early (T1/T2) squamous cell cancers of the oral cavity and oropharynx.

The overall methodology for performing radioguided SLN biopsy for squamous cell cancers of the head and neck region, including the oral cavity, oropharynx, hypopharynx, and laryngeal region is not that dissimilar as to that for breast cancer and melanoma. However, there are some unique features that are worth further discussing [392397].

Recently, Vigili et al [396] have outlined one such protocol for performing radioguided SLN biopsy for squamous cell cancers of the oral cavity and oropharynx. Topical anesthetic ( $10 \%$ lidocaine spray) was administered to the oral cavity. Then, $0.8 \mathrm{mCi}(30 \mathrm{MBq})$ to $1.4 \mathrm{mCi}(50 \mathrm{MBq})$ of ${ }^{99 \mathrm{~m} T c}$ human nanocolloidal albumin within $0.3 \mathrm{ml}$ of normal saline solution was injected superficially into the subepithelial stroma in four points around the tumor. Injection into deeper tissues resulted in a poorer image quality, increased blood accumulation of the tracer, and a lower success rate for SLN localization. The mouth was immediately washed out in order to prevent pooling and swallowing of any residual ${ }^{99 \mathrm{~m} T c}$ human nanocolloidal albumin. Immediate dynamic lymphoscintigraphy imaging and 30 minute static images were performed with lateral and/or anterior views. The skin overlying the area of the identifiable SLNs seen on lymphoscintigraphy were marked with a permanent marking pen. Subsequent appropriate surgery and radioguided SLN biopsy with the gamma detection probe were performed approximately three hours after completion of lymphoscintigraphy. They defined a SLN as any lymph node containing activity counts of at least three times that of the background count activity.

Likewise, recently, Tomifuji et al [397] have outlined one such protocol for performing radioguided SLN biopsy for squamous cell cancers of the hypopharynx and laryngeal region. On the day before surgery, topical anesthetic ( $4 \%$ lidocaine spray) was administered to the oral cavity. Then, a $2.0 \mathrm{mCi} / \mathrm{mL}(74 \mathrm{MBq} / \mathrm{mL})$ solution of ${ }^{99 \mathrm{~m} T c}$ phytate was submucosally injected in $0.2 \mathrm{~mL}$ quantities into three to four sites adjacent to the tumor using a 23-gauge endoscopic puncture needle that was passed through the channel of a flexible laryngohypopharyngeal endoscope with a transparent hood. Three hours after the injection, static lymphoscintigraphy images were obtained from lateral and anterior views. The skin overlying the area of the identifiable SLNs seen on lymphoscintigraphy were marked with a permanent marking pen. The following day, appropriate surgery and radioguided SLN biopsy with the gamma detection probe were performed. They defined a SLN as any lymph node containing activity counts of at least ten times that of the background count activity.

The determination of the adequacy of the intraoperative assessment of the neck with the gamma probe during radioguided SLN biopsy for squamous cell cancers of the oral cavity and oropharynx has been recently assessed. In a 
recent study of 31 patients undergoing radioguided SLN biopsy, resection of the primary tumor, and a concomitant neck dissection, Atula et al [398] reported that all patients could be accurately staged based upon the findings within the hottest three SLNs removed.

Despite encouraging results, controversy still exists as to whether SLN biopsy will ultimately replace the standard clinical practice of selective neck dissection for squamous cell cancers of the head and neck region, as previously described $[399,400]$. Potential disadvantages of SLN biopsy include a limited exposure and the potential injury to neural structures, such as cranial nerve XI and the mandibular branch of cranial nerve VII [401]. Additionally, if neck nodal metastases are not identified with frozen section, then a subsequent operation within a recently open and inflamed surgical field could increase morbidity [393]. Furthermore, it has been observed that lymph nodes predominately replaced by metastatic disease do not accumulate radiotracer and may result in altered lymphatic pathways, thereby increasing false negatives [402]. Finally, the proximity of the primary tumor and the SLN can be problematic. Kovács et [401] al reported that 6 of 104 known sites of radiotracer localization seen on preoperative lymphoscintigraphy were intraoperatively missed using the gamma detection probe secondary to shinethrough radioactivity from a nearby injection site. This proximity issue likely explains why the radioguided SLN identification frequency is less for carcinomas of the floor of mouth as compared to other sites of carcinomas within the oral cavity, oropharynx, hypopharynx, and laryngeal region [392,395-397].

Regardless of these potential disadvantages, radioguided SLN biopsy has proven value especially in management of the No neck in squamous cell cancers of the head and neck region $[278,392,395,396]$. One advantage of radioguided SLN biopsy is for guiding selective nodal harvesting in carcinomas situated at or adjacent to the midline, since in such situations the lymphatic drainage may be bilateral in up to $10 \%$ of cases [403]. In this setting, lymphoscintigraphy and intraoperative gamma probe detection of SLNs may provide a suitable alternative in these patients, in whom the management of the contralateral neck is debatable. Another advantage of SLN biopsy is the allowance of a concentrated and extensive pathologic examination of a limited number of lymph nodes versus that of a limited pathologic examination of numerous lymph nodes from selective neck dissections where finding a metastasis is similar to the proverbial needle in a haystack [392]. Because of this required meticulous histopathologic examination, Kovács [404] has discouraged the use of frozen section, but admits its intraoperative availability and diagnostic accuracy are important in shortening the time to deciding on a therapeutic neck dis- section. SLN biopsy has been reported to aid in the identification of skip metastases, defined by Byers et al [405] as metastases that bypass level I and II lymph nodes and go directly to levels III through V. They demonstrated that skip metastases were the only manifestation of disease in the neck after selective neck dissection in approximately $16 \%$ of 277 patients evaluated. Such a finding can clearly explain disease relapse after surgeries which do not include all five levels. A final potential benefit of radioguided SLN biopsy is the upstaging of early tumors from NO to N1 which occurs in $16 \%$ to $34 \%$ [392,395] of patients with early squamous cell carcinomas (T1/2) of the oral cavity and oropharynx. This finding has led to appropriate therapy of the neck (i.e., neck dissection or radiation), and unlike in melanoma where lymphatic metastases portend an extremely poor prognosis, squamous cell carcinomas of the head and neck region with lymphatic metastases remain potentially curable.

\section{RIGS}

Only one available report can be found in the literature on the application of RIGS to squamous cell cancers of the head and neck region [406]. In this report, Argenzio et al described the intravenous administration of $15 \mathrm{mCi}(555$ $\mathrm{MBq})$ of $99 \mathrm{mTc}-\mathrm{labeled}$ anti-CEA monoclonal antibody fragments in two cases with squamous cell cancer of the head and neck, with one to the cheek and one to the scalp [406]. Diagnostic gamma camera imaging was performed 4 and 12 hours after intravenous administration of $99 \mathrm{mTc}$ labeled anti-CEA monoclonal antibody fragments and then intraoperative gamma probe detection was used to assist in the resection of the primary tumor and the assessment of surgical margins at a time approximately 36 hours after the initial intravenous dosage of this radiopharmaceutical. They defined tumor to healthy tissue background ratio of greater than two as a discriminate value for defining diseased tissue.

\section{F-FDG-directed surgery}

Only one report is currently available in the literature on the application of gamma probe detection during ${ }^{18} \mathrm{~F}$ FDG-directed surgery to squamous cell cancer of the head and neck region [45]. In this report, Gulec et al [45] describe a single case in which ${ }^{18} \mathrm{~F}$-FDG-directed surgery was used the localize a nonpalpable target from a head and neck cancer at approximately 4 hours after intravenous injection of a unknown dose of 18 F-FDG. In an additional report by Meller et al [44], they describe the use of a high energy gamma detection probe to preoperatively identify metastatic lymph nodes in 36 patients with cancers of the oral cavity and oropharynx which was performed within a two week period prior to their definitive surgery and specifically at the time of their diagnostic whole body PET scan. Patients were intravenously injected with $6.8 \mathrm{mCi}(250 \mathrm{MBq})$ to $9.5 \mathrm{mCi}(350 \mathrm{MBq})$ 
of ${ }^{18} \mathrm{~F}-\mathrm{FDG}$ for the purpose of the preoperative diagnostic whole body PET scan and for the formalized preoperative survey of the lymph node levels within the bilateral neck with a high energy gamma detection probe. Nevertheless, this high energy gamma detection probe was not utilized within the intraoperative setting during their definitive surgical procedure.

\section{Parathyroid disease}

Aside from radioguided SLN biopsy for breast cancer and melanoma, the gamma detection probe has not been utilized more frequently in any other arena than it has in minimally-invasive radioguided parathyroid surgery for primary hyperparathyroidism. The traditional surgical approach to primary hyperparathyroidism has included bilateral neck exploration with visualizing all parathyroid tissue and removing the enlarged gland(s). In the hands of an experienced parathyroid surgeon, this approach has a success rate of over 95\% [407]. However, since a single adenoma is responsible for at least $85 \%$ of all cases of primary hyperparathyroidism, the use of bilateral neck exploration has been deemed by some surgeons to represent vast over treatment of such cases. Coupled with advances in preoperative localization of parathyroid tissue with preoperative $99 \mathrm{mTc}-\mathrm{MIBI}$ imaging and intraoperative quick parathyroid hormone assay, minimallyinvasive radioguided parathyroid surgery has become a viable and widely accepted alternative to that of traditional bilateral neck exploration in appropriately selected cases.

\section{Minimally-invasive radioguided parathyroidectomy for primary hyperparathyroidism secondary to parathyroid adenomas}

In 1984, Ubhi et al [408] first described the use of the gamma detection probe for intraoperative identification of $201 \mathrm{Tl}$-thallous chloride in a case of mediastinal parathyroid adenoma (Table 1). Then, in 1995, Martinez et al [409] first described the use of the gamma detection probe for intraoperative identification of ${ }^{99 \mathrm{~m} T \mathrm{Tc}-\mathrm{MIBI} \text { in patients }}$ with parathyroid gland pathology (Table 1). Later in 1997, the University of South Florida group $[410,411]$ popularized this technique of using ${ }^{99 \mathrm{mTC}-\mathrm{MIBI} \text { for the }}$ surgical management of primary hyperparathyroidism (Table 1). Since that time, multiple other groups of investigators have published reports describing their experience with minimally-invasive radioguided parathyroidectomy for primary hyperparathyroidism [412-431].

In their initial report, Norman and Chheda from The University of South Florida [410] described their technique of minimally-invasive radioguided parathyroidectomy in 15 patients and utilized an approach of same-day MIBI imaging and radioguided parathyroid surgery. Patients were intravenously injected with 20 to $25 \mathrm{mCi}$ (740 to 925
MBq) of ${ }^{99 m T C-M I B I ~[411] . ~ A f t e r ~ c o m p l e t i o n ~ o f ~ p r e o p e r a-~}$ tive scintigraphic localization of the presumed parathyroid adenoma (at approximately 3 hours after the MIBI injection), patients underwent radioguided parathyroid surgery. A gamma detection probe was then used to systematically assess radioactivity in all four quadrants of the neck. An initial $2 \mathrm{~cm}$ skin incision was then made overlying the location of the radioactive parathyroid gland, the platysma muscle and strap muscles were retracted, and dissection proceeded with guidance from the gamma detection probe for identification of the radioactive parathyroid gland representing the presumed parathyroid adenoma. After resection of the radioactive gland, counts were recorded of the four quadrants of the neck, along with excised radioactive parathyroid gland, excised fat, and excised lymph nodes. Parathyroid adenomas were consistently found to have counts exceeding the postexcisional background counts by more than $20 \%$, whereas the counts of fat and lymph nodes never exceeded $3 \%$ of the postexcision background counts [411]. This finding has subsequently led to the abandonment of frozen section pathology for confirmation of the presence of parathyroid tissue [411]. In their initial report [410], a single parathyroid adenoma was located by this technique in 14 of 15 patients, with an average time of approximately 19 minutes to find the radioactive parathyroid gland and an average total operating time of approximately 48 minutes. The one failed patient was intraoperatively found to have fourgland hyperplasia as diagnosed by increased background counts after successful excision of the first targeted radioactive parathyroid gland. The first 5 procedures were done under general anesthesia, with all subsequent procedures being done under local anesthetic.

Instead of utilizing a same-day protocol for MIBI imaging and radioguided parathyroid surgery, other investigators have described and recommended utilizing separate days for MIBI imaging and radioguided parathyroid surgery in order to allow for more efficient use of operative time by preselecting those individuals with confirmed localization to a solitary parathyroid adenoma on MIBI imaging for determination of the appropriateness of minimallyinvasive radioguided parathyroidectomy $[412,419]$. Flynn et al [412] previously described performing preoperative MIBI imaging electively before the day of surgery and subsequent intravenous injection of $20 \mathrm{mCi}(740 \mathrm{MBq})$ of $99 \mathrm{mTc}-\mathrm{MIBI}$ on the day of surgery at a time approximately 60 to 90 minutes preoperatively. In order to decrease the radiation dose at the time of surgery, Rubello and colleagues have developed a low dose 99mTc-MIBI technique [418-424]. As originally described, Rubello et al [419] reported performing preoperative double-tracer $\left({ }^{99 \mathrm{mTc}} \mathrm{Tc}\right.$


time several days before the proposed surgery to identify those individuals with a presumed solitary parathyroid 
adenoma and then, on the day of surgery, those individuals who preoperatively localized were subsequently intravenously injected with a low dose of $1 \mathrm{mCi}(37 \mathrm{MBq})$ of 99mTc-MIBI just a few minutes prior to the start of surgery. Both the Flynn et al series [412] and the Rubello et al series [419] reported excellent intraoperative localization rates with the gamma detection probe for demonstrating an abnormal parathyroid gland. Additionally, with the low dose 99mTc-MIBI technique that was administered just a few minutes prior to the start of surgery, Rubello et al [419] demonstrated that all excised parathyroid adenomas had ex vivo radioactivity that was more than $40 \%$ of the postexcision background counts, further confirming completeness of surgical excision beyond that of the $20 \%$ rule previously established by Murphy and Norman [411]. Finally, both the Flynn et al series [412] and the Rubello et al series [419] used an intraoperative quick parathyroid hormone assay to aid in the surgical decisionmaking process. While Rubello and colleagues [419422,424] and Chen et al [428] have strongly advocated the routine use of the intraoperative quick parathyroid hormone assay for further confirming completeness of removal of all hyperfunctioning parathyroid tissue (especially to aid in the recognition of previously unrecognized double adenomas or multiple-gland hyperplasia), Flynn et al [412], Goldstein et al [427], Caudle et al [429], and Norman and Politz [430] have not. In their series of 112 patients with preoperative localization on an MIBI scan, Goldstein et al [427] reported a 98\% success rate for identification of a radioactive parathyroid gland based upon the intraoperative use of the gamma detection probe alone during minimally-invasive radioguided parathyroid surgery.

Several authors have discussed the potential advantages of a minimally-invasive radioguided parathyroid surgical approach as compared to standard bilateral neck exploration for the surgical management of primary hyperparathyroidism. Rubello and colleagues [418-424] have emphasized several potential advantages of a minimallyinvasive radioguided parathyroid surgical approach for the surgical management of primary hyperparathyroidism. This includes minimizing the invasiveness of the surgical approach for identifying and resecting solitary parathyroid adenomas (thus allowing for maximal cosmetic outcome and the ability to perform such cases without the need of general anesthesia), as well as enhancing the accuracy of the surgeon to specifically locate ectopic parathyroid adenomas and ensure complete operative success by intraoperatively evaluating the postresectional field for residual radioactivity. Additionally, Flynn et al [412] has emphasized a modest cost savings of almost one thousand dollars per patient, particularly due to shorter operative time, avoidance of general anesthesia, elimination of the need for both frozen section pathology and intraoperative quick parathyroid hormone assays, and earlier hospital discharge. A similar effect on cost was reported by Goldstein et al. [413] in which they described a reduction in hospital charges by nearly $50 \%$ for the minimally-invasive radioguided parathyroid surgical approach as compared to that of standard neck exploration. Furthermore, a potential benefit of a minimallyinvasive radioguided parathyroid surgical approach for reoperative parathyroid surgery has been reported in cases where a failed initial surgical exploration without the use of the gamma detection probe resulted from what was labeled as a "false-positive" MIBI scan [414]. In this series of 17 patients in which a minimally-invasive radioguided parathyroid surgical approach was utilized for reoperative parathyroid surgery, Norman et al [414] reported that a repeat MIBI scan again demonstrated the same focus of radioactivity and intraoperative use of the gamma detection probe correctly identified and aided in the excision of a radioactive parathyroid gland in all 17 patients who were previously presumed to have an initial "false-positive" MIBI scan.

Throughout the development of minimally-invasive radioguided parathyroid surgery, identification of an adenoma on preoperative MIBI scintigraphic imaging has been the most important criteria for the determination of the appropriateness of this surgical approach. However, if an initial negative MIBI scan is encountered in a patient with primary hyperparathyroidism, there may still be a viable role for consideration of MIBI-directed intraoperative gamma probe detection at the time of minimallyinvasive radioguided parathyroid surgery. Lal and Chen [431] recently described an algorithm for patients with primary hyperparathyroidism in whom an initial negative MIBI scan is encountered. In a group of 90 such patients, this algorithm involved the use of further preoperative testing with thallium subtraction scanning and neck ultrasound, as well as intraoperative bilateral internal jugular sampling of parathyroid hormone, along with intraoperative quick parathyroid hormone assay, and/or intraoperative MIBI-directed gamma probe detection. Their results indicated that despite an initial negative MIBI scan, that $67 \%$ of such patients had a single adenoma as the cause of their primary hyperparathyroidism, and $23 \%$ of such patients had successful utilization of intraoperative MIBIdirected gamma probe detection at the time of minimallyinvasive radioguided parathyroid surgery.

Radioguided parathyroidectomy for hyperparathyroidism secondary to hyperplastic parathyroid glands

While the role of utilizing intraoperative gamma probe detection during radioguided parathyroid surgery is well established for parathyroid adenomas, few reports have focused on its effectiveness for hyperplastic parathyroid glands demonstrated in either primary or secondary/terti- 
ary hyperparathyroidism [432-440]. This approach was first successfully reported in 2000 by Rossi et al [432] in 11 patients with persistent hyperparathyroidism undergoing reoperative neck exploration for localizing residual hyperfunctioning parathyroid tissue and by Navarra et al [433] in a single patient with recurrent secondary renal hyperparathyroidism. The largest series to date has been reported by Chen et al [436], in which they compared the results of minimally-invasive radioguided parathyroid surgery in 25 patients with secondary/tertiary hyperparathyroidism with that of 77 patients with primary hyperparathyroidism. Their results demonstrated in vivo counts of parathyroid adenomas and hyperplastic parathyroid glands did not significantly differ; however, ex vivo counts were highest in single gland adenomas and lowest in hyperplastic parathyroid glands. Nevertheless, all hyperplastic parathyroid glands still registered ex vivo counts > $20 \%$ of postexcision background counts in a setting where each patient received an intravenous dosage of $10 \mathrm{mCi}$ (370 MBq) of ${ }^{99 m T c-M I B I}$ at approximately 30 to $60 \mathrm{~min}-$ utes before surgery. Despite this, Rubello et al [424] still recommend use of the intraoperative quick parathyroid hormone assay at the time of radioguided parathyroidec-

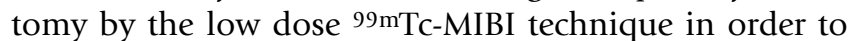
establish the completeness of removal of additional foci of hyperfunctioning parathyroid tissue secondary to the persistence of previously unrecognized hyperplastic parathyroid glands.

\section{Radioguided surgical approach to recurrent parathyroid cancer}

Parathyroid cancer is an extremely rare entity that has been reported to occur in $0.1 \%$ to $0.4 \%$ of patients with primary hyperparathyroidism [441]. The incidental finding of parathyroid cancer at the time of minimally-invasive radioguided surgery for primary hyperparathyroidism presumed secondary to a parathyroid adenoma is welldescribed in the literature [424]. Nevertheless, only one report in the literature exists which describes the use of an intraoperative gamma probe detection of ${ }^{99 \mathrm{mTc}-\mathrm{MIBI} \text {-avid }}$ tissue during radioguided surgery that is specifically directed toward parathyroid cancer [442]. In this report, they describe a case of recurrent parathyroid carcinoma in which the patient was intravenously injected with $10 \mathrm{mCi}$ (370 MBq) of ${ }^{99 \mathrm{mTC}-M I B I}$ at one hour prior to surgery and an intraoperative gamma detection probe was used to localize and aid in the resection of the area of recurrent disease. In this case, successful resection was verified by normalization of the postresection parathyroid hormone level on intraoperative quick parathyroid hormone assay and for which they report that the patient remains asymptomatic at 17 months after surgery.

\section{Thyroid cancer}

Radioguided surgery for thyroid cancer has gained some attention in recent years. While radioguided SLN biopsy has been limitedly investigated [443], the mainstay of radioguided surgery for thyroid cancer has been directed towards the utilization of iodine-based and $99 \mathrm{mTc}$-labeled radiopharmaceutical agents for identifying recurrent disease in both differentiated thyroid cancer and medullary thyroid cancer.

Despite the standard practice of using total thyroidectomy and selective ${ }^{131}$ I remnant ablative therapy for the initial therapeutic strategy for differentiated thyroid cancer, locoregional neck recurrence occurs in approximately $5 \%$ to $20 \%$ of such patients [444-446]. The application of additional courses of ${ }^{131}$ I ablative therapy alone has not proven adequate for the control of disease recurrence of differentiated thyroid cancer [444]. In this regard, radioguided surgery offers a mechanism to achieve complete tumor extirpation of recurrent or persistent disease with a relatively high degree of sensitivity and specificity. Such a technique is important in aiding in the detection of occult disease not readily apparent from a preoperative diagnostic evaluation that may be closely approximated to vascular structures or that is found within areas of scar tissue and sclerosis resulting from previous surgeries, external beam radiation therapy, or high-dose ${ }^{131}$ I ablative therapy [446]. This approach allows for the systematic evaluation of the completeness of surgical resection in extended operations for recurrent or persistent disease, particularly in anatomically difficult areas [447].

Numerous radiopharmaceutical agents have been described for radioguided surgery for recurrent thyroid cancer. The most logical choices for recurrent differentiated thyroid cancer would include ${ }^{131} \mathrm{I}$ and ${ }^{123} \mathrm{I}$. However, a previous history of ${ }^{131}$ I ablative therapy has been shown to abolish subsequent uptake of radioiodine in approximately $65 \%$ of previously ablated patients with recurrent differentiated thyroid cancer [448]. These iodine-negative recurrent tumors, being resistant to further ${ }^{131}$ I therapy, carry a worse prognosis, with reported 2-, 5-, and 10-year survival rates of $55 \%, 16 \%$, and $11 \%$, respectively, compared to $91 \%, 77 \%$, and $62 \%$, respectively, for patients with iodine-avid recurrent tumors $[449,450]$. In this regard, $65 \%$ of patients with iodine-negative metastases have disease that is generally limited to the neck and/or mediastinum. For such patients, the only effective treatment is radical surgery, which can result in a complete, sustained remission in up to $50 \%$ of cases [451], confirming the importance of early recognition of recurrent differentiated thyroid cancer, as well as the importance of assessing whether it is limited to one or more organ systems. In this regard, radioguided surgery is a potentially powerful mechanism for managing patients with iodinenegative metastases in whom a more aggressive treatment strategy is required. 
Radioguided surgery of iodine-avid recurrent differentiated thyroid cancer and recurrent medullary thyroid cancer with iodine radionuclides

The experience of treating differentiated thyroid cancers with ${ }^{131}$ I remnant ablative therapy following total thyroidectomy has made ${ }^{131}$ I the most logical radionuclide for localization of iodine-avid recurrent tumors during radioguided surgery. Due to the fact that ${ }^{131}$ I has a relatively long physical half-life (approximately 8 days), as well as the fact that it is readily available, highly affordable [26], and can be used to distinguish tumor from that of background with a collimated gamma detection probe, it is an ideal radionuclide for detecting iodine-avid recurrent disease. This approach for using ${ }^{131} \mathrm{I}$ is well reported in the literature. The first description of radioguided surgery for thyroid cancer was published in 1956 by Harris et al [3] and was later refined in 1971 by Morris et al [452], in which they described using a CsI [Tl] gamma detection probe to localize thyroid tissue or recurrent thyroid cancer in patients undergoing neck exploration.

Recently, Rubello et al [446] reported an eight-day protocol for radioguided surgery for iodine-avid recurrent disease, which was modified from the first original protocol reported by Travagli et al [453]. In this protocol, a therapeutic dosage of $100 \mathrm{mCi}(3700 \mathrm{MBq})$ of ${ }^{131}$ I was orally administered to hypothyroid patients (serum thyroid stimulating hormone level $>30 \mu \mathrm{U} / \mathrm{ml}$ ) on day 0 , with a whole-body gamma camera imaging ( ${ }^{131}$ I scan) performed on day 3 . Then on day 5 , radioguided neck surgery was performed with the assistance of a $15-\mathrm{mm}$ collimated handheld gamma detection probe, and all sites of elevated activity were resected. A subsequent postoperative neck ${ }^{131}$ I scan was obtained on day 7 to evaluate the success of the surgery, utilizing the remaining radioactivity from the initial oral dosage of ${ }^{131}$ I given on day 0 . Of the 184 metastatic foci found within the 31 treated patients, $41.3 \%$ of extirpated metastatic foci were localized only with the gamma detection probe and were not seen with preoperative imaging. The postoperative neck ${ }^{131}$ I scan on day 7 showed a negative pattern in 25 of 31 treated patients $(80.6 \%)$. As such, the remaining 6 treated patients showed reduced ${ }^{131}$ I uptake, suggesting persistent iodine-avid residual disease. Alternative ${ }^{131}$ I dosage regimens and different timing regimens for radioguided surgery have been reported. Negele et al [447] reported using a diagnostic dosage of ${ }^{131} \mathrm{I}$ in the range of 1.9 to $9.5 \mathrm{mCi}$ ( 70 to $350 \mathrm{MBq}$ ) and radioguided neck surgery performed at 6 to 8 days after the original ${ }^{131}$ I dosage. Their utilization of a significantly lower diagnostic dosage of ${ }^{131} \mathrm{I}$, rather than a therapeutic dosage $(100 \mathrm{mCi})$, avoided the need for hospitalization prior to the planned radioguided neck surgery [447]. Furthermore, Scurry et al [454] reported that radioguided neck surgery was possible for up to three weeks after the administration of a therapeutic dosage of ${ }^{131} \mathrm{I}$.

In an effort to shorten the time interval between radionuclide administration and the time to radioguided surgery for iodine-avid recurrent thyroid cancer, Gallowitsch et al [455] reported using ${ }^{123}$ I instead of ${ }^{131}$ I for radioguided surgery for recurrent papillary thyroid cancer using an oral ${ }^{123}$ I dosage of $2 \mathrm{mCi}(74 \mathrm{MBq})$. The short physical half-life of approximately 13 hours for ${ }^{123}$ I allows it to be administered on the day of or on the day prior to the planned radioguided surgical procedure for an iodine-avid recurrent thyroid cancer $[455,456]$. Unlike ${ }^{131} \mathrm{I},{ }^{123} \mathrm{I}$ is a pure gamma photon emitter, is not associated with the potential stunning effect on subsequent radioiodine uptake, and produces less radiation exposure to the patient and the surgeon [456]. This concept of using ${ }^{123}$ I has also been similarly applied to recurrent medullary thyroid cancer by Shimotake at al [457] in which they reported intravenously administering ${ }^{123} \mathrm{I}-\mathrm{MIBG}$ at a dosage of $2.7 \mathrm{mCi}$ (100 MBq) at a time 24 hours prior to the planned radioguided surgical procedure. Despite these potentially favorable reasons for utilizing ${ }^{123} \mathrm{I}$ instead of ${ }^{131} \mathrm{I}$, the greater expense and relatively limited availability of ${ }^{123}$ I has contributed to the more popular continued use of ${ }^{131}$ I for radioguided surgery for iodine-avid recurrent differentiated thyroid cancers.

\section{Radioguided surgery of iodine-negative recurrent differentiated thyroid cancer and recurrent medullary thyroid cancer with $99 \mathrm{mTc}$ labeled radiopharmaceutical agents, I'In-labeled radiopharmaceutical agents, and 18 F-FDG}

In contrast, radioguided surgery of iodine-negative recurrent differentiated thyroid cancer and recurrent medullary thyroid cancer has focused primarily upon the use of $99 \mathrm{mTc}$-labeled radiopharmaceutical agents, ${ }^{111}$ In-labeled radiopharmaceutical agents, and ${ }^{18} \mathrm{~F}-\mathrm{FDG}$. While ${ }^{99 \mathrm{mTc}} \mathrm{T}$ MIBI [458], 99mTc-dimercaptosuccinic acid ( $99 \mathrm{~m} \mathrm{Tc}(\mathrm{V})$ DMSA) [459], and 99mTc-tetrafosmin [460] have all been utilized for diagnostic gamma camera imaging of iodinenegative recurrent differentiated thyroid cancers, only 99mTc-MIBI and $99 \mathrm{mTc}(\mathrm{V})$-DMSA have been described for gamma detection probe-directed radioguided surgery.

Rubello et al [461] reported on 37 patients who underwent a previous total thyroidectomy and subsequent ${ }^{131}$ I ablative therapy for differentiated thyroid cancer and who later demonstrated evidence of an iodine-negative recurrence. Each such patient had an elevated thyroglobulin level, a negative 131I scan, demonstration of recurrent locoregional recurrent disease on a preoperative 99mTcMIBI scan and on a high-resolution ultrasound, and no demonstration of distant metastatic disease [461,462]. Then, each patient was taken to the operating suite and, 10 minutes prior to starting the procedure, was intrave- 
nously injected with $1 \mathrm{mCi}(37 \mathrm{MBq})$ of 99mTc-MIBI. A gamma detection probe was then used to guide resection of all foci of $99 \mathrm{mTc}$-MIBI uptake. In total, 66 discrete nodules in 37 patients were identified by both high-resolution ultrasound and intraoperative gamma probe detection and were subsequently successfully resected [461]. At follow-up ranging from 9 to 57 months, 27 of 37 patients remained disease-free. In contrast to other approaches, the use of $99 \mathrm{~m}$ Tc-MIBI and intraoperative ultrasound for radioguided surgery of recurrent thyroid cancers is relatively far less expensive and far more available to most medical facilities.

Just as $99 \mathrm{mTC}-\mathrm{MIBI}$ has proven benefit for the management of recurrent differentiated thyroid cancer, $99 \mathrm{mTc}(\mathrm{V})$-DMSA has been shown to be effective in the diagnostic evaluation of recurrent medullary thyroid cancer, with a detection sensitivity of 95\% [459]. Adams et al [463] reported on medullary thyroid cancer recurrences in 25 patients evaluated by preoperative diagnostic tumor localization imaging by computed tomography, ${ }^{111}$ In-DTPA-D-Phe ${ }^{1}$ octreotide imaging, and 99mTc-(V)-DMSA imaging, as well as by intraoperative surgical palpation and by radioguided surgery using an intraoperative gamma probe detection system. All patients were preoperatively injected with an intravenous dose of $6 \mathrm{mCi}(222 \mathrm{MBq})$ of ${ }^{111} \mathrm{In}-\mathrm{DTPA}-\mathrm{D}-$ Phe ${ }^{1}$-octreotide and an intravenous dose of $13.5 \mathrm{mCi}$ (500 MBq) of 99mTc-(V)-DMSA [463]. ${ }^{111}$ In-DTPA-DPhe ${ }^{1}$-octreotide imaging was performed 4 hours and 24 hours after injection. ${ }^{99 m T c-(V)-D M S A}$ imaging was performed 6 hours after injection. Radioguided surgery was performed approximately 24 hours after ${ }^{111}$ In-DTPA-DPhe ${ }^{1}$-octreotide injection and approximately 6 hours after 99mTc-(V)-DMSA injection. They demonstrated lesion detection sensitivities of 32\%, 34\%, and 65\% for preoperative diagnostic tumor localization imaging by computed tomography, ${ }^{111}$ In-DTPA-D-Phe ${ }^{1}$-octreotide imaging, and 99mTc-(V)-DMSA imaging, respectively [463]. In addition, lesion detection sensitivities were $65 \%$ by intraoperative surgical palpation and $97 \%$ by radioguided surgery using a combination of channel selection for both ${ }^{111}$ In and ${ }^{99 \mathrm{mT}} \mathrm{Tc}$ on the gamma probe detection unit. In this series, radioguided surgery detected metastases as small as $5 \mathrm{~mm}$ in greatest dimension, whereas intraoperative surgical palpation detected metastases only greater than or equal to 1 $\mathrm{cm}$ in greatest dimension. Likewise, radioguided surgery was able to identify more than $30 \%$ more foci of recurrent medullary thyroid carcinoma compared with conventional preoperative diagnostic tumor localization imaging and intraoperative surgical palpation. Despite these highly encouraging results with recurrent medullary thyroid cancer, ${ }^{99 \mathrm{mTc}} \mathrm{T}(\mathrm{V})$-DMSA is no longer commercially available for use.
As mentioned above, ${ }^{111}$ In-DTPA-D-Phe ${ }^{1}$-octreotide has been investigated in radioguided surgery for recurrent medullary thyroid cancer [463]. However, the overall sensitivity of detecting metastases from recurrent medullary thyroid cancer on diagnostic ${ }^{111}$ In-DTPA-D-Phe ${ }^{1}$-octreotide imaging [463] is far less than the overall sensitivity of detecting metastases from iodine-negative recurrent differentiated thyroid cancer on diagnostic ${ }^{111}$ In-DTPA-DPhe $^{1}$-octreotide imaging (34\% versus 74\%, respectively) [464]. Despite the better overall sensitivity of detecting metastases from iodine-negative recurrent differentiated thyroid cancer on diagnostic ${ }^{111}$ In-DTPA-D-Phe ${ }^{1}$-octreotide imaging, there is no published data on the use of ${ }^{111}$ In-DTPA-D-Phe ${ }^{1}$-octreotide imaging for radioguided surgery for iodine-negative recurrent differentiated thyroid cancer.

${ }^{111}$ In RIGS has been limitedly investigated for recurrent medullary thyroid cancer in France [465-467]. These studies have used a two-step radioimmunotargeting system, consisting of a bispecific antibody (composed of a fragment of anti-CEA monoclonal IgG1 that is coupled chemically with a fragment of anti-DTPA monoclonal IgG1) and an ${ }^{111}$ In-labeled bivalent hapten $\left({ }^{111}\right.$ In-di-DTPAtyrosyl-lysine). Patients were first intravenously injected with $0.1 \mathrm{mg} / \mathrm{kg}$ of body weight of the bispecific antibody. Approximately three to five day later, patients were then injected with $2.7 \mathrm{mCi}$ to $10 \mathrm{mCi}$ (100 MBq to $370 \mathrm{MBq}$ ) of the ${ }^{111}$ In-labeled bivalent hapten. RIGS was then performed approximately two to four days later. Using this RIGS protocol for detecting recurrent and metastatic medullary thyroid cancer, de Labriolle-Vaylet et al [467] most recently reported an accuracy of $86 \%$, a sensitivity of $75 \%$, and a specificity of $90 \%$ and suggested its value in the surgical management of this disease process.

The use of ${ }^{18} \mathrm{~F}-\mathrm{FDG}$ for gamma probe-directed radioguided surgery of iodine-negative recurrent thyroid tumors has been recently investigated in a limited fashion $[42,45,47,468-470]$. It is well-established that ${ }^{18 F-F D G}$ PET imaging is particularly useful in the detection of iodine-negative recurrent differentiated thyroid cancer with reported sensitivities ranging from $85 \%$ to $95 \%$ $[471,472]$ and a specificity as high as 90\% [472].

In 2005, Kraeber-Bodéré et al [42] first reported on the use of $18 \mathrm{~F}$-FDG-directed radioguided surgery in 10 patients with iodine-negative recurrent differentiated thyroid cancer. All 10 patients had previously undergone a diagnostic preoperative ${ }^{18} \mathrm{~F}-\mathrm{FDG}$ PET/CT scan demonstrating abnormal hypermetabolic activity suggesting cervical recurrence, but with no evidence of distant disease. Then, on the day of surgery, at approximately 30 minutes prior to the start of the surgical procedure, patients were injected with a mean dose of $7.2 \mathrm{mCi}(265 \mathrm{MBq})$ of ${ }^{18 F-F D G}$ and 
a subsequent intraoperative radioguided survey of the operative field was performed with a handheld gamma detection probe. Likewise, a completion lymph node dissection was performed in all patients after the intraoperative radioguided survey. A total of 12 abnormal findings were demonstrated on both ${ }^{18}$ F-FDG PET/CT and on the intraoperative radioguided survey, with no additional abnormal finding demonstrated on the intraoperative radioguided survey with the gamma detection probe. Based on the results of the completion lymph node dissection that were performed on all of the patients, they demonstrated that diagnostic preoperative ${ }^{18} \mathrm{~F}$-FDG PET/CT scan and intraoperative radioguided survey failed to identify all sites of additional lymph node microscopic metastases in five of the patients.

More recently, Gulec et al [45] reported on four cases of iodine-negative recurrent thyroid cancer undergoing radioguided surgery among a series of 25 patients with various malignancies [45]. In this series, patients were injected with an intravenous dose of 5 to $15 \mathrm{mCi}$ (185 to 555 $\mathrm{MBq}$ ) of ${ }^{18} \mathrm{~F}-\mathrm{FDG}$ at approximately 2 to 6 hours prior to the planned surgical procedure. Subsequent radioguided surgery was performed with successful localization of nonpalpable metastatic lymph nodes and other target tissues.

Most recently, Agrawal et al [470] reported on two cases of iodine-negative recurrent thyroid cancer at The Ohio State University in which a multimodality approach of perioperative ${ }^{18} \mathrm{~F}-\mathrm{FDG}$ PET/CT imaging (preoperative patient imaging, specimen imaging, and postoperative patient imaging), intraoperative gamma probe detection, and intraoperative ultrasound were utilized for tumor localization and resection of all sites of hypermetabolic activity. The two patients were intravenously injected with 15 to $20 \mathrm{mCi}$ (555 to $740 \mathrm{MBq}$ ) of ${ }^{18} \mathrm{~F}-\mathrm{FDG}$ at approximately 120 minutes prior to the planned time of surgery and this multimodality approach allowed for successful identification and resection of all occult sites of iodine-negative recurrent thyroid cancer.

\section{Radioguided SLN biopsy}

The utility of SLN biopsy for differentiated thyroid cancer remains a topic of major debate. Although it is reported that up to $80 \%$ of differentiated thyroid cancer patients have neck lymph node metastases when a lymphadenectomy accompanies the total thyroidectomy [473], only a minority ( $3 \%$ to $30 \%$ ) of patients will later develop recurrent neck disease $[473,474]$. Furthermore, in an extensive literature review, Grebe and Hay [474] concluded that nodal disease at presentation was not a predictor of patient survival for papillary thyroid cancer. As a result, the current management of the central neck compartment lymph nodes consists of intraoperatively identifying sus- picious lymph nodes, followed by central compartment and superior mediastinal nodal clearance [475].

In the current literature, only a limited number of studies have been published on the feasibility of radioguided SLN biopsy for thyroid cancer [443,476-482]. Those available studies report success of localization in the range of $78 \%$ to $100 \%$. In those studies, $0.11 \mathrm{mCi}$ to $3.2 \mathrm{mCi}(4 \mathrm{MBq}$ to $120 \mathrm{MBq}$ ) of ${ }^{99 \mathrm{~m} T \mathrm{~T}}$ colloidal human albumin was injected intratumorally in a total volume of $0.1 \mathrm{~mL}$ to $0.5 \mathrm{~mL}$ of normal saline. Preoperative lymphoscintigraphy was generally performed. The hemithyroidectomy or total thyroidectomy was generally performed first, followed by the radioguided SLN biopsy procedure, in order to minimize the "shine through" effect which can be especially problematic in the central neck compartment where the lymph nodes may be otherwise within close proximity to the thyroid gland [476]. Despite these encouraging results, it still remains unclear as to whether radioguided SLN biopsy will prove to be of any prognostic value in the management of differentiated thyroid cancer.

\section{Parotid gland cancer}

Although the generalized concept of a so-called "sentinel node" was first coined by Ernest Gould in 1960 in parotid gland cancer [483], only one report exists within the literature on radioguided SLN biopsy for parotid gland cancer [484], and this single report also represents the only paper addressing any aspect of radioguided surgery for this disease entity. In 2006, Stárek et al [484] reported on six patients injected in eight divided portions into the parotid gland equidistantly from the tumor with approximately $1.35 \mathrm{mCi}(50 \mathrm{MBq})$ of ${ }^{99 \mathrm{~m} T c}$ colloidal human albumin in a total volume of $2 \mathrm{~mL}$. These patients subsequently underwent preoperative lymphoscintigraphy, radioguided SLN biopsy, and elective lymph node dissection. A SLN was detected in all six cases. In two cases, preoperative lymphoscintigraphy and radioguided SLN biopsy revealed additional sentinel lymph nodes outside the usual extent of elective lymph node dissection that is generally performed for parotid gland cancer. One false-negative (i.e., a positive SLN outside of the parotid gland) was seen with demonstration of intraparotid localization only and was explained by obstruction of the lymphatic outflow from the parotid gland by an intraparotid SLN metastasis.

\section{Gynecologic malignancies}

Vulvar cancer

The application of the gamma detection probe in radioguided surgery for vulvar cancer has been limited to radioguided SLN biopsy. Since its first description by Decesare et al in 1997 [485], the feasibility of radioguided SLN biopsy for vulvar cancer has been well evaluated and reported in the literature [268,269,485-509]. In general, 99mTc sulfur 
colloid or ${ }^{99 \mathrm{mTC}}$ colloidal human albumin have been utilized and injected into two to four intradermal sites around the primary tumor in a total dosage range from $0.4 \mathrm{mCi}(15 \mathrm{MBq})$ to $4.0 \mathrm{mCi}(150 \mathrm{MBq})$. In the six most recently published series, localization has been reported in $93 \%(\mathrm{n}=40)$ by Nyberg et al [501], 98\% $(\mathrm{n}=62)$ by Vidal-Sicart et al [502], 95\% $(\mathrm{n}=41)$ by Hauspy et al [503], 100\% $(\mathrm{n}=43)$ by Rob et al [504], 95\% $(\mathrm{n}=39)$ by Johann et al [508], and $98 \%(n=127)$ by Hampl et al [509], with false negative results reported in $0 \%, 0 \%, 0 \%$, $0 \%, 2.2 \%$, and $7.7 \%$ of patients, respectively. Bilateral groin localization has been reported in $19 \%$ to $39 \%$ [493,500,502], emphasizing the importance of preoperative lymphoscintigraphy for most effectively performing radioguided SLN biopsy in vulvar cancer. Currently, there is a multi-institutional clinical trial (GOG173) for SLN biopsy for vulvar cancer from the collaborative Gynecologic Oncology Group, starting in December 1999 and as of September 2007, has enrolled 456 patients from 40 sites [510]. Since 2001, radioguided SLN biopsy became a mandatory component for enrollment into GOG173 and 316 such patients have been enrolled with $96 \%$ localization accomplished using the combined radiocolloid and vital blue dye technique. However, a full disclosure of the results of GOG173 is still pending completion of patient enrollment and publication of the data.

\section{Vaginal carcinoma}

Radioguided surgery using the gamma detection probe for vaginal carcinoma has been limited to three small case report series addressing radioguided SLN biopsy $[490,511,512]$. In the largest such radioguided SLN biopsy series reported in 2004 by van Dam et al [511], four patients with vaginal carcinoma received a dose of approximately $1.6 \mathrm{mCi}(60 \mathrm{MBq})$ of $99 \mathrm{mTc}$ colloidal human albumin injected into the normal vaginal mucosa (at a depth of 4 to $5 \mathrm{~mm}$ ) in four locations around the vaginal tumor. Preoperative lymphoscintigraphy was performed and patients with verified localization were subsequently taken to the operating room for laparoscopic radioguided sentinel lymph node biopsy approximately three to five hours after the initial ${ }^{99 \mathrm{~m} T c}$ colloidal human albumin injection. Localization on preoperative lymphoscintigraphy was demonstrated in three of four (75\%) cases. Laparoscopic radioguided SLN biopsy was successful in all three vaginal carcinomas showing localization on preoperative lymphoscintigraphy.

\section{Cervical cancer}

The application of the gamma detection probe in radioguided surgery for cervical cancer has been limited to radioguided SLN. Since its first description by Verheijen et al [513] and Kamprath et al [514] in 2000, the feasibility of radioguided SLN biopsy for cervical cancer has been well

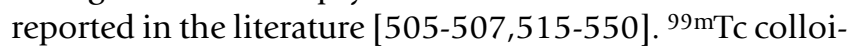

dal human albumin, ${ }^{99 \mathrm{mTC}}$ sulfur colloid, and ${ }^{99 \mathrm{mTC} \text { nano- }}$ colloid have all been used as the radiocolloid. The range of time for injection of radiocolloid varies from intraoperative, to immediately preoperative, and to as long as 24 hours prior to the planned surgical procedure. Most commonly, a total dose of the radiocolloid, ranging from 0.5 $\mathrm{mCi}(18.5 \mathrm{MBq})$ to $3.2 \mathrm{mCi}(120 \mathrm{MBq})$, has been recommended for injection into the four quadrants of the cervix, while attempting to avoid direct intratumoral injection.

In most series, a sentinel lymph node was generally identified in anywhere from $80 \%$ to $100 \%$ of patients, a SLN being positive for metastatic disease in $8 \%$ to $30 \%$ of cases, and false-negative rates were generally reported as approximately $8 \%$ with ranges of $0-20 \%$ [505-507,515$550]$. The finding of bilateral sentinel lymph nodes is generally described in approximately half of the patients, and in as high as $70 \%$ of patients in some series. Interestingly, two of the most recently reported series have demonstrated an improved detection rate in primary tumors that are $\leq 2 \mathrm{~cm}[549,550]$.

The Arbeitsgemeinschaft Gynäkologische Onkologie (AGO, German Association of Gynecologic Oncologist) has recently published the largest series to date on radioguided SLN in cervical cancer [549]. In this multicenter prospective validation series, patients generally received $1.6 \mathrm{mCi}(60 \mathrm{MBq})$ of ${ }^{99 \mathrm{~m} T \mathrm{Tc}}$ nanocolloid injected into four sites beneath intact-appearing cervical epithelium on the day before surgery and $4 \mathrm{~mL}$ of blue dye injected intraoperatively after induction of anesthesia in a similar manner. Data from a total of 590 patients were used for analysis of the detection rate and data from a total of 507 patients were used for analysis of the diagnostic accuracy. The overall detection rate of pelvic SLNs was $88.6 \%$. The overall sensitivity was only $77.4 \%$, and this was significantly less $(\mathrm{P}<0.001)$ than the predefined acceptability mark of $\geq 90 \%$. Nevertheless, the sensitivity in women with tumors $\leq 20 \mathrm{~mm}$ was $90.9 \%$, and bilateral detection was noted in $87.2 \%$ of such patients. The overall negative predictive value was $94.3 \%$ and negative predictive value was significantly higher $(\mathrm{P}<0.001)$ in patients with tumors $\leq$ $20 \mathrm{~mm}(99.1 \%)$ as compared with patients with tumors $>$ $20 \mathrm{~mm}(88.5 \%)$ The authors concluded that due to the suboptimal overall sensitivity of $77.4 \%$ that complete pelvic lymphadenectomy cannot yet be omitted at this time in the surgical management of cervical cancer. Nevertheless, they commented that SLN biopsy should continue to be investigated in future studies in patients with tumors $\leq$ $2 \mathrm{~cm}$ [549].

Finally, the Gynecologic Oncology Group (GOG) has completed a prospective trial (GOG 206) to determine the false negative rate in a large randomized prospective trial in cervical cancer patients. However, their results have not 
yet been officially reported. [507,551,552]. In this regard, multiple authors have continued to advocate ongoing prospective evaluation of SLN biopsy before its routine use in the surgical management of cervical cancer can be more universally advocated [505-507,551-554].

\section{Endometrial cancer}

The application of the gamma detection probe in radioguided surgery for endometrial cancer has been limited to radioguided SLN biopsy. Since its first description by Pelosi et al in 2002 [555], the feasibility of radioguided SLN biopsy for endometrial cancer has been well reported in the literature [505-507,556-574]. Most frequently, $99 \mathrm{mTc}$ colloidal human albumin has been used as the radiocolloid. However, $99 \mathrm{mTc}$ sulfur colloid and ${ }^{99 \mathrm{mT}} \mathrm{Tc}$ phytate have also been utilized. The range of time for injection of radiocolloid varies from intraoperative, to immediately preoperative, and to as long as 24 hours prior to the planned surgical procedure.

Two major injection techniques have been described for the injection of the radiocolloid for endometrial cancer. This includes injection into the uterine corpus and injection into the cervix. Those authors who injected into the cervix [555-557,560,562,564,569-572] usually injected a total dose of the radiocolloid ranging from $1.0 \mathrm{mCi}(37$ $\mathrm{MBq})$ to $3.24 \mathrm{mCi}(120 \mathrm{MBq})$ into the four quadrants of the cervix to a depth from $0.5 \mathrm{~cm}$ to $1.5 \mathrm{~cm}$ on the day prior to the surgical procedure. Those authors who injected into the uterine corpus usually inject a total dose of radiocolloid ranging from $0.8 \mathrm{mCi}(30 \mathrm{MBq}$ ) to 3.0 $\mathrm{mCi}(111 \mathrm{MBq})$ into the corpus of the uterus using several different schedules and techniques of injection $[558,559,561,563,566-568,572]$. Most commonly this is accomplished hysteroscopically by injecting the radiocolloid in a subendometrial fashion in juxtaposition to the tumor at approximately 2 to 6 hours prior to proceeding to the operating room for the staging procedure $[561,563,572]$. Variations of this technique include those by Nikura et al $[559,568]$ in which they inject radiocolloid hysteroscopically into the endometrium on the day before the surgical procedure and by Delaloye [567] in which they inject radiocolloid hysteroscopically into the endometrium in the operating room immediately prior to starting the staging procedure. Finally, one group [566] attempted to inject into the subserosal myometrium of the uterine fundus at three midline sites at the time of the staging procedure, but achieved only limited success.

In most series, a SLN was generally identified in anywhere from $70 \%$ to $100 \%$ of patients, a SLN being positive for metastatic disease in $14 \%$ to $25 \%$ of cases, and false-negative rates were generally reported as $5 \%$ or less [505507,555-574].
It is important to note, that only one prospective randomized clinical trial exists in the literature for endometrial cancer [572] which compared the cervical injection approach $(\mathrm{n}=23)$ to the hysteroscopic subendometrial injection approach $(\mathrm{n}=17)$ for radiocolloid. There was no difference in the SLN detection rates for the cervical injection technique $(70 \%)$ versus the hysteroscopic subendometrial injection technique (65\%). Likewise, no falsenegative results were seen in either group. Interestingly, this study showed that there were no identifiable aortic SLNs in the cervical injection group as compared to finding aortic SLNs in $11.8 \%$ of patients receiving a hysteroscopic subendometrial injection.

\section{Ovarian Cancer}

Although radioguided surgery is not routinely utilized in the current surgical management of ovarian cancer, the application of the gamma detection probe for ovarian cancer has been limitedly applied to the radioguided detection of occult primary disease and recurrent disease. No data are available on radioguided SLN biopsy for ovarian cancer. However, RIGS [149,575-582] and ${ }^{18 F-F D G-~}$ directed surgery $[45,53,583]$ applications of the gamma detection probe have been investigated.

\section{RIGS}

Since first described for ovarian cancer by Martin et al in $1988[149,578]$, the feasibility of RIGS has been evaluated by several groups of investigators [149,575-582]. The focus of all these RIGS protocols has been the detection of occult recurrence in those ovarian cancer patients undergoing both second look laparotomy and those undergoing surgery for suspected or confirmed recurrence. Martin et al $[149,578]$ evaluated ${ }^{125}$ I-B72.3 murine monoclonal antibody, McIntosh et al [582] evaluated 125I-CC49 murine monoclonal antibody, and Krag et al [580] evaluated ${ }^{111}$ I-B72.3 murine monoclonal antibody. Gitsch et al [575-577] and Jäger et al [579] evaluated 131I-OC125 (anti-CA125) murine monoclonal antibody. Finally, Ind et al [581] evaluated ${ }^{99 \mathrm{~m} T \mathrm{Tc}}$ labeled murine monoclonal antibodies SM3 and H17E2. Each of these RIGS protocols utilized similar regimens with intravenous injection of 2 to $5 \mathrm{mCi}$ ( 74 to $185 \mathrm{MBq}$ ) of the radiolabeled monoclonal antibody at an interval of 4 to 42 days prior to the planned surgical procedure [149,575-582]. The reported detection rates for RIGS in these reports ranged from $50 \%$ to $80 \%$. Despite these early promising results, no further investigations into RIGS for ovarian cancer have been made in over the last 10 years.

\section{${ }^{18}$ F-FDG-directed surgery}

Most recently, ${ }^{18}$ F-FDG-directed surgery has been evaluated in the setting of recurrent ovarian cancer $[45,53,583]$. In 2005, Barranger et al [583] first described ${ }^{18} \mathrm{~F}-\mathrm{FDG}$ directed surgery in a single patient case report for a patient 
undergoing laparoscopic excision of recurrent ovarian cancer using a ${ }^{18} \mathrm{~F}-\mathrm{FDG}$ dose of $0.11 \mathrm{mCi} / \mathrm{kg}(4 \mathrm{MBq} / \mathrm{kg})$. The most recent and largest report to date is by Cohn et al [53] at The Ohio State University. Three patients undergoing laparotomy for recurrent ovarian cancer underwent preoperative intravenous injection of approximately 10 to $20 \mathrm{mCi}$ (370 to $740 \mathrm{MBq}$ ) of ${ }^{18} \mathrm{~F}-\mathrm{FDG}$ at approximately 60 to 120 minutes prior to the planned surgical procedure. A combined approach of intraoperative gamma probe detection, specimen PET/CT imaging, and postoperative PET/CT patient imaging was utilized, and demonstrated the feasibility of this approach for establishing the location and extent of disease and confirming complete cytoreduction in selected cases of recurrent ovarian cancer.

\section{Urologic malignancies \\ Penile cancer}

The application of the gamma detection probe in radioguided surgery for penile cancer has been limited to radioguided SLN biopsy. Nevertheless, the routine use of radioguided SLN biopsy for penile cancer remains controversial and is yet to be widely adopted [584].

The generalized concept of a so-called "sentinel node" in our modern medical literature was first coined by Ernest Gould in 1960 in parotid gland cancer [483] and was then later most notably attributed to the work of Ramon Cabañas in 1977 in the area of penile squamous cell carcinoma [585], which was based upon Ramon Cabañas' careful work and meticulous description of lymphadenography of the dorsal lymphatics of the penis [586]. Gould's [483] and Cabañas' [585] strict definition of the "sentinel node" was based on its proximity to a static anatomical landmark (i.e., the angle of the confluence of the anterior and posterior facial veins and the superficial epigastric vein, respectively) and was not based on actual variations in the pattern of lymphatic drainage. In penile cancer, based upon utilizing the superficial epigastric vein as a static anatomical landmark for identifying the socalled "sentinel node" of penile cancer, multiple groups reported variable results and very high false-negative rates [587-591]. Resultantly, controversy has continued about whether the concept of SLN biopsy is reliable in the management of the regional lymph nodes in penile cancer.

It was not until 1993, after Krag et al [135] described the utilization of radioguided SLN biopsy in breast cancer, that a re-emerging interest developed in the concept of SLN biopsy for penile cancer. Since 2000, multiple reports have been published evaluating the feasibility and efficacy of radioguided SLN biopsy for penile cancer [592-609]. In

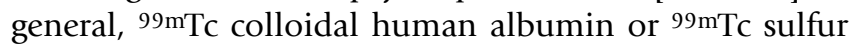
colloid and have been utilized and injected into one to four intradermal or subdermal sites around the primary tumor in a total dosage range from $0.5 \mathrm{mCi}(19 \mathrm{MBq})$ to $3.2 \mathrm{mCi}(120 \mathrm{MBq})$.

In the four largest published series on penile cancer in which a subsequent formal lymph node dissection was performed only if a SLN was positive, radioguided localization was reported in $97 \%(\mathrm{n}=74)$ by Valdés Olmos et al [594], 98\% $(\mathrm{n}=90)$ by Tanis et al [597], 98\% by Kroon et al [599], and $96 \%(n=75)$ by Hadway et al [607]. Bilateral groin localization was reported in $81 \%, 79 \%, 80 \%$, and $91 \%$, respectively [594,597,599,607], emphasizing the extreme importance of preoperative lymphoscintigraphy for most effectively performing accurate radioguided SLN biopsy in penile cancer. A SLN was positive in $22 \%$, $20 \%$, 21\%, and $25 \%$ of patients, respectively $[594,597,599,607]$. In this regard, at a median follow-up of 28 months, 7 months, and 11 months, respectively, only $3.6 \%, 6.3 \%$, and $1.9 \%$ of patients with an initial negative SLN biopsy developed a nodal metastasis in a previously SLN-negative nodal basin [594,599,607]. Despite these encouraging results, the routine use of radioguided SLN biopsy for penile cancer remains controversial within the field of urological surgery and may not become widely adopted until there is verification of this technology by a large prospective randomized clinical trial [584].

\section{Prostate cancer}

The gamma detection probe is not routinely used in the current surgical management of prostate cancer. Nevertheless, radioguided SLN biopsy has been thoroughly evaluated for its potential application in the surgical management of prostate cancer and RIGS has been previously very limitedly investigated.

\section{Radioguided SLN biopsy}

The utilization of radioguided SLN biopsy for prostate cancer was first reported in 1999 by Wawroschek et al [610]. Since that time, multiple reports have been published on the potential application of radioguided SLN biopsy in the surgical management of prostate cancer [611-632]. Most frequently, 99mTc colloidal human albu-

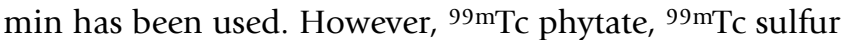

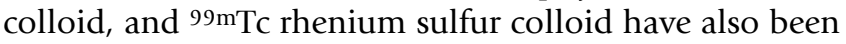
utilized. Patients undergo the radiocolloid injection at an interval from 6 hours to 24 hours before the planned surgery by an ultrasound-guided transrectal approach and into one to three sites within the peripheral zone of the prostate gland. Total dosage range for the radiocolloid is from $1.6 \mathrm{mCi}(60 \mathrm{MBq})$ to $10.8 \mathrm{mCi}(400 \mathrm{MBq})$, which is injected in a total volume of normal saline from $0.2 \mathrm{~mL}$ to $3.0 \mathrm{~mL}$. In most series, a SLN was generally identified in $90 \%$ to $100 \%$ of patients, a SLN was positive in $12 \%$ to $25 \%$ of cases, and false negative rates were generally reported as $5 \%$ or less. 
The largest radioguided SLN biopsy series reported to date was recently published by Weckermann and associates from Augsburg, Germany [627]. In their report, they describe 1,055 patients with clinically organ confined prostate cancer undergoing radioguided SLN biopsy, pelvic lymph node dissection, and radical retropubic prostatectomy. A SLN was detected in all patients. A positive SLN were found in 205 patients (19.4\%), and only two false negative cases $(<1 \%)$ were encountered. One to three positive lymph nodes were found in 95, 50, and 21 cases, respectively, and more than 3 positive lymph nodes were found in 41 cases. Approximately 63\% of patients having positive lymph nodes had such positive lymph nodes found outside the region of the standard pelvic lymphadenectomy field. Therefore, Weckermann et al [627] strongly recommended that patients with a positive SLN should undergo extended pelvic lymphadenectomy rather than standard pelvic lymphadenectomy since, in such cases, tumor cells were also additionally detected in non-SLNs.

Finally, several series have reported upon the feasibility of laparoscopic radioguided SLN biopsy [622,624,629,632]. Such a minimally-invasive diagnostic approach to the lymph nodes in the management of prostate cancer may ultimately prove valuable in the evaluation of the lymph node status in patients with an intermediate or high risk of nodal metastases who are either being considered for external beam radiation therapy or for robotic prostatectomy. However, no prospective randomized data are available at this time to more fully address this question.

\section{RIGS}

The investigation of RIGS in prostate cancer has been limited to a single report on 125I-B72.3 monoclonal antibody and a single report on ${ }^{111}$ In-capromab pendetide. In 1993, Badalament et al [633] from The Ohio State University reported their RIGS feasibility study from ten patients with prostate cancer who were planned for radical retropubic prostatectomy and bilateral pelvic lymphadenectomy and that were intravenously injected with $2 \mathrm{mCi}$ (74 $\mathrm{MBq}$ ) of ${ }^{125} \mathrm{I}-\mathrm{B} 72.3$ monoclonal antibody at an interval from 17 to 38 days prior to the anticipated date of surgery. RIGS successfully localized tumor in all ten patients, identified otherwise occult bilateral intraprostatic tumor in three patients, correctly identified regional lymph node metastases in two patients, and demonstrated no falsenegative results. In 2000, Anderson et al [634] reported a single case of suspected metastatic prostate cancer in which the patient was intravenously injected with $5 \mathrm{mCi}$ (185 MBq) of ${ }^{111}$ In-capromab pendetide. Four days later, the patient underwent RIGS with surgical excision of a RIGS-positive lymph node in the left supraclavicular region that confirmed the diagnosis of metastatic prostate cancer. Despite these two early reports on RIGS in prostate cancer, no further investigations have occurred since that time.

\section{Testicular cancer}

The application of the gamma detection probe in radioguided surgery for testicular cancer has been only limitedly investigated in regards to radioguided SLN biopsy and ${ }^{18} \mathrm{~F}$ FDG-directed surgery.

\section{Radioguided SLN biopsy}

To date, only three reports exist in the literature on radioguided SLN biopsy for testicular cancer [635-637]. The technique of laparoscopic radioguided SLN biopsy was first described in 2002 for clinical stage I testicular cancer by Tanis et al [635] and by Ohyama et al [636].

In a series of 5 patients, Tanis et al described the technique of laparoscopic radioguided SLN biopsy in two patients [635]. All patients received an intratesticular injection of 1.4 to $3.7 \mathrm{mCi}$ ( 52 to $135 \mathrm{MBq}$ ) of ${ }^{99 m T c}$ colloidal human albumin in 0.15 to $0.3 \mathrm{ml}$ of normal saline and underwent subsequent lymphoscintigraphy. The last two patients underwent same-day transperitoneal laparoscopic radioguided SLN biopsy, with identification of two radioactive SLNs in one patient (with no nodal metastases identified) and no radioactive SLNs identified in the second patient.

In a series of 15 patients, Ohyama et al [636] described the technique of laparoscopic radioguided SLN biopsy in five patients. The day before surgery, each patient was injected into the testicular tissue around the tumor in the testicular tunica albuginea with $0.2 \mathrm{mCi}(7.5 \mathrm{MBq})$ of ${ }^{99 \mathrm{mTc}}$ phytate in $0.2 \mathrm{ml}$ of normal saline and underwent subsequent lymphoscintigraphy. The following day, patients one through ten underwent extraperitoneal laparoscopic retroperitoneal lymph node dissection, patients 11 through 14 underwent extraperitoneal laparoscopic radioguided SLN biopsy (with 11 to 14 SLNs removed in each patient and with no nodal metastases identified), and patient 15 underwent extraperitoneal laparoscopic radioguided SLN biopsy with a subsequent confirmatory extraperitoneal laparoscopic retroperitoneal lymph node dissection (with the finding to two micrometastatic disease in SLNs and no metastatic disease in any non-SLNs). Ohyama et al [636] simply concluded that extraperitoneal laparoscopic radioguided SLN biopsy is technically feasible, but requires further clinical study.

Most recently in 2005, Satoh et al [637] reported the most comprehensive series on radioguided SLN biopsy in 22 patients with clinical stage I testicular cancer. The day before surgery, each patient was injected into the testicular tissue around the tumor in the testicular tunica albuginea with $0.4 \mathrm{mCi}(15 \mathrm{MBq})$ of ${ }^{99 \mathrm{mTc}}$ phytate in $0.4 \mathrm{ml}$ of nor- 
mal saline and underwent subsequent lymphoscintigraphy. The following day, patients underwent extraperitoneal laparoscopic radioguided SLN biopsy with a subsequent confirmatory extraperitoneal laparoscopic retroperitoneal lymph node dissection. Radioactive SLNs were identified in 21 of 22 patients (95\%), with one radioactive SLN identified in 15 patients and 2 to 4 radioactive SLN identified in the six other patients. Only two patients $(9.5 \%)$ had micrometastatic disease in radioactive SLNs. Likewise, none of the 21 patients in which a radioactive SLN was identified had metastatic disease in any non-SLNs harvested at the time of the subsequent confirmatory extraperitoneal laparoscopic retroperitoneal lymph node dissection. Two patients with no metastatic disease in their radioactive SLNs or in their subsequent confirmatory extraperitoneal laparoscopic retroperitoneal lymph node dissection developed lymph node disease in the paraaortic lymph nodes at the level of the renal vein and in the obturator lymph nodes at 10 and 20 months after surgery, respectively. Satoh et al [637] concluded that extraperitoneal laparoscopic radioguided SLN biopsy is technically feasible and has potential value in staging and treatment of patients with early-stage testicular cancer. Nevertheless, the lack of any other subsequent reports in the literature or the mention of radioguided SLN biopsy in the National Comprehensive Cancer Network (NCCN) clinical guidelines for testicular cancer [638] leads one to conclude that this is not yet considered an accepted standard of care.

\section{${ }^{18} \mathrm{~F}$-FDG-directed surgery}

There is a single reported case in the literature by Gulec et al [43] for using ${ }^{18} \mathrm{~F}$-FDG-directed surgery for the identification and resection of a hypermetabolic metastatic periaortic lymph node in a case of seminoma.

\section{Bladder cancer}

Within the published literature, the application of the gamma detection probe in radioguided surgery for bladder cancer has been limited to only a small number of reports from Sweden on radioguided SLN [639-642]. In all instances, ${ }^{99 \mathrm{~m} T c}$ colloidal human albumin has been utilized and injected into four peritumoral sites within the detrusor muscle in a total dosage range from $1.4 \mathrm{mCi}(50$ MBq) to $1.9 \mathrm{mCi}(70 \mathrm{MBq})$. In 2001, Sherif et al [639] were the first to report on 13 bladder cancer patients in which localization was seen in 11 of 13 patients $(85 \%)$, with four patients having a positive SLN, and with no false-negative results. In 2003 Liedberg et al [640] reported on 26 bladder cancer patients in which localization was seen in 21 of 26 patients (81\%), with seven patients having a positive SLN, and with one false-negative result. In 2006, Liedberg et al [641] reported on 75 bladder cancer patients in which localization was seen in 65 of 75 patients $(87 \%)$, with 26 patients having a posi- tive SLN, and with six false-negative results (19\%). Despite the higher false-negative rate reported by Liedberg et al [641], a common theme to all these reports has been that approximately one-quarter of all patients that localized had a SLN that was anatomically located outside the field of the normally excised lymph nodes of the obturator fossa, thus leading to improved overall lymph node staging when radioguided SLN biopsy was used in conjunction with standard lymphadenectomy. Finally, Sherif et al [642] demonstrated that the use of fused single-photon emission computed tomography (SPECT) and computed tomography (CT) preoperative imaging aided in the intraoperative identification of both metastatic and non-metastatic SLNs.

\section{Renal cell cancer}

Only two reports involving cases of renal cell cancer can be found within the published literature that focus on the use of the gamma detection probe in radioguided surgery $[29,643]$. Both reports involve RIGS with the utilization of monoclonal antibodies. However, only one of these two reports describes the use of a particular monoclonal antibody that is specific to renal cell carcinoma [29].

Recently, in 2008, Strong et al [29], reported on two cases of clear-cell renal cell cancer in which RIGS was performed using the monoclonal antibody cG250 and ${ }^{124}$ I (Table 1). cG250 is well known to bind with a high specificity to clear-cell renal cell cancers [644-646]. The specific antigen target of cG250 is an epitope of carbonic anhydrase IX $[29,646]$. In these two RIGS cases in the Strong et al series [29], the patients were each intravenously injected with 5 mCi (187 MBq) of ${ }^{124}$ I-labeled cG250 approximately seven days prior to surgery. PET imaging was performed four hours post-injection of ${ }^{124}$ I-labeled cG250 and then again approximately seven days later at a time specifically within three hours of the start of surgery. Intraoperatively, both a gamma detection probe and a beta detection probe were utilized for tumor identification. No significant difference in the ratio of tumor/background counts were found for the two different types of radiation detection probes utilized.

The incidental finding of metastatic renal cell cancer at the time of RIGS performed for a presumed primary colon cancer was previously reported by Avital et al in 1998 [643]. In this report, a 72 year old female with a remote history of renal cell cancer requiring a right nephrectomy some five years earlier and who was now presumed to have a primary colon cancer underwent RIGS after receiving an intravenous injection of $2 \mathrm{mCi}(74 \mathrm{MBq})$ of $125 \mathrm{I}-$ labeled murine CC49 monoclonal antibody approximately three weeks prior to surgery. After pathologic evaluation of the case, it was determined that the colonic mass was, in fact, a metastatic renal cell cancer deposit that had 
metastasized to and through all layers of the colonic wall. Immunohistochemistry revealed that the tumor cells composing the metastatic renal cell cancer were themselves negative for CC49 monoclonal antibody staining. However, the normal colonic mucosa adjacent to the metastatic renal cell cancer demonstrated strong CC49 monoclonal antibody staining. Therefore, in retrospect, the adjacent normal colonic mucosa and not the remotely occurring metastatic renal cell cancer deposit was responsible for the accumulation of the 125I-labeled murine CC49 monoclonal antibody detected at the time of RIGS.

\section{Thoracic malignancies}

Lung cancer

The use of a gamma detection probe in lung cancer dates back to 1984 when Woolfenden et al [647] first described using a miniature $\mathrm{NaI}[\mathrm{Tl}]$ detector during fiberoptic bronchoscopy to attempt to detect and localize sites of deposit of ${ }^{57} \mathrm{Co}$ bleomycin in 34 patients with suspected lung cancer. Despite the promising results of this report, the extremely long physical half-life of ${ }^{57} \mathrm{Co}$ (271.8 days) has made it a highly undesirable radionuclide for any further use in radioguided surgical procedures. Since that time, radioguided surgery for lung cancer has been further investigated using $99 \mathrm{mTc}$ for radioguided SLN biopsy, using 125I-labeled and $99 \mathrm{mTc}$-labeled monoclonal antibodies for RIGS, as well as using $99 \mathrm{mTC}$-peplomycin and ${ }^{18} \mathrm{~F}-\mathrm{FDG}$ for other radioguided surgical applications.

\section{Radioguided SLN biopsy}

The utilization of radioguided SLN biopsy for non-small cell lung cancer was first reported in 2000 by Liptay et al [648]. Since that time, multiple reports have been published on the potential application of radioguided SLN biopsy in the surgical management of non-small cell lung cancer [649-668]. 99mTc tin colloid, 99mTc sulfur colloid, and ${ }^{9} 9 \mathrm{~m} T \mathrm{Tc}$ colloidal human albumin have all been utilized in radioguided sentinel lymph node biopsy for non-small cell lung cancer. Some authors have advocated preoperative injection of radiocolloid into or around the primary tumor by CT guidance on the day of surgery or on the day before surgery. Other authors have advocated intraoperative injection of radiocolloid into or around the primary tumor after direct visualization of the tumor. While still other authors advocate intraoperative endobronchial injection of radiocolloid into directly visualized endobronchial tumor or transbronchially at the most distal pulmonary sub-segment that could be reached endobronchially within proximity to the primary tumor. Total dosage range for the radiocolloid is from $0.25 \mathrm{mCi}(9.25$ $\mathrm{MBq}$ ) to $8 \mathrm{mCi}$ (296 MBq), which is injected in a total volume of normal saline from $0.5 \mathrm{~mL}$ to $2.0 \mathrm{~mL}$. In all series reporting more than one hundred patients undergoing radioguided SLN biopsy for non-small cell lung cancer $[657,659,662,668]$, a SLN was generally identified in $70 \%$ to $100 \%$ of patients and false negative rates were generally reported as $10 \%$ or less. Despite these numerous reports and their encouraging results, the routine use of radioguided SLN biopsy in the surgical management of non-small cell lung cancer has not been embraced. Nevertheless, those authors who have played an active role in its evaluation in the surgical management of non-small cell lung cancer remain optimistic about its future applications [669].

\section{RIGS}

The application of RIGS technology to adenocarcinoma of the lung has been previously investigated and reported in an extremely limited fashion [670,671]. In 1998, Grazia et al [670] intraoperatively evaluated 9 patients with adenocarcinoma of the lung with the gamma detection probe after intravenous injection of 0.9 to $1.5 \mathrm{mCi}$ (33 to 56 $\mathrm{MBq}$ ) of ${ }^{125}$ I-labeled monoclonal antibody B72.3 at 11 to 44 days (mean of 20.4 days) prior to the surgical procedure. Specific binding to histologically confirmed sites of adenocarcinoma of the lung was noted in all such cases. However, the high radioactive background counts found in the thorax, due to the heart and major vessels, was postulated as a drawback to further investigation of this technique. Likewise, in 1998, Mansi et al [671], evaluated both ${ }^{125}$ I-labeled monoclonal antibody B72.3 and $99 \mathrm{mTc}-$ labeled fragments of the murine anti-CEA monoclonal antibody F023C5 in lung cancer patients. In the first phase of their study, using immunohistochemistry, they found binding of monoclonal antibody B72.3 in only 6 of 45 primary non-small cell lung cancers. Only one operable patient was then intravenously injected with $2 \mathrm{mCi}$ (74 MBq) of ${ }^{125}$ I-labeled monoclonal antibody B72.3 and no selectivity for neoplastic cells was seen during RIGS using the gamma detection probe. In the second phase of their study, 11 patients with squamous cell lung cancer were intravenously injected with $15 \mathrm{mCi}(555 \mathrm{MBq})$ of ${ }^{99 \mathrm{~m} T c}$-labeled fragments of the murine anti-CEA monoclonal antibody F023C5. Immunoscintography was then performed six to 24 hours thereafter. Only one patient was operable and underwent RIGS in concert with a pneumonectomy and lymphadenectomy at a time some 36 hours after the original intravenous injection of the ${ }^{99 \mathrm{mTc}}$ labeled fragments of the murine anti-CEA monoclonal antibody F023C5. Using the gamma detection probe, in vivo detection of the primary tumor was not accomplished; however, resected neoplastic tissue demonstrated a 2:1 tumor-to-background ratio on ex vivo counting. Likewise, RIGS identified a small lymph node metastasis that was not previously identified by CT scan or immunoscintigraphy. Despite these two early reports on RIGS in lung cancer, no further investigations have occurred since that time. 


\section{9mTc-peplomycin radioguided surgery}

In 2003, Wang et al [672] reported on a series of 37 patients with lung neoplasms who were intravenously injected with a non-specified dose of $99 \mathrm{~m} T \mathrm{~T}$-peplomycin. Peplomycin is a semi-synthetic analog of bleomycin. Following injection, patients underwent preoperative scintigraphic imaging for identification of radiotracer uptake by tumor-bearing tissue and were then subsequently taken to the operating room for radioguided surgery using the gamma detection probe. The sensitivity, specificity, and accuracy was reported as $90 \%, 88 \%$, and $89 \%$, respectively, for identifying malignant lung lesions, and was reported as $91 \%, 88 \%$, and $90 \%$, respectively, for identifying lymph node metastases.

\section{${ }^{18}$ F-FDG-directed surgery}

Much more recently, the application of intraoperative gamma probe detection after a preoperative same-day injection of ${ }^{18} \mathrm{~F}-\mathrm{FDG}$ has been reported in a limited number of selected cases of non-small cell lung cancer $[45,673,674]$. First reported in 2006, Nwogu et al [673] evaluated 10 patients with non-small cell lung cancer that were intravenously injected with approximately $10 \mathrm{mCi}$ (370 MBq) of ${ }^{18}$ F-FDG on the day of surgery. All resected primary tumors were FDG-avid. With regards to evaluation of the thoracic lymph nodes, there were 5 cases of true-positive FDG-avid thoracic lymph nodes, 3 cases of false positive FDG-avid thoracic lymph nodes, and 2 falsenegative cases in which the thoracic lymph nodes were not FDG-avid but were found to contain metastatic disease. In this regard, and of most recent note, Moffatt-Bruce et al at The Ohio State University [674] has recently reported a combined approach for radioguided localization of FDG-avid tissues for a single case of lung cancer using perioperative ${ }^{18 F-F D G ~ P E T / C T ~ i m a g i n g ~(u s i n g ~ a ~}$ triad of preoperative PET/CT imaging, specimen PET/CT imaging, and postoperative PET/CT imaging) and intraoperative gamma probe detection, in which a dose of approximately $26.1 \mathrm{mCi}$ (966 MBq) ${ }^{18} \mathrm{~F}-\mathrm{FDG}$ was injected intravenously approximately 98 minutes prior to the start of radioguided localization of FDG-avid tissues.

\section{Pulmonary nodules}

The application of the gamma detection probe for the intraoperative localization and video-assisted thoracoscopic wedge resection of solitary or multiple small indeterminate pulmonary nodules has been investigated [675683]. Generally, on the morning of surgery, such pulmonary lesions are injected under CT guidance with $99 \mathrm{mTc}$ colloidal human albumin in dosage range from $0.14 \mathrm{mCi}$ (5 MBq) to $0.30 \mathrm{mCi}(11 \mathrm{MBq})$ and in a total volume of normal saline from $0.1 \mathrm{~mL}$ to $0.3 \mathrm{~mL}$. Subsequently, such patients are taken to the operating room for video-assisted thoracoscopic wedge resection of these previously injected pulmonary nodules. In the largest and most recent study conducted to date, Grogan et al (683) reported that pulmonary nodules were successfully localized and excised by this radioguided technique in 77 of 81 patients (95\%), and were exclusively thoracoscopically excised in 71 of 77 patients (92\%).

\section{Neuroendocrine tumors \\ Gastroenteropancreatic (GEP) neuroendocrine tumors}

While GEP neuroendocrine tumors generally follow a protracted clinical course, surgery remains integral in the management of localized primary tumors, recurrent disease, or metastatic disease [684-687]. Therefore, the ability to detect and localize GEP neuroendocrine tumor tissue is paramount to the effectiveness of surgical treatment and was revolutionized by Krenning's landmark description of radiolabeled octreotide scintigraphy using ${ }^{111}$ In-DTPA-D-Phe ${ }^{1}$-octreotide and ${ }^{123}$ I-Tyr $^{3}$-octreotide, which both specifically target somatostatin receptors [688]. As a result, ${ }^{111}$ In-DTPA-D-Phe ${ }^{1}$-octreotide imaging has been coupled with single photon emission computerized tomography (SPECT) to become the primary imaging and staging modality of patients with GEP neuroendocrine tumors, exceeding the sensitivity of MRI and CT $[689,690]$. Despite this well-established preoperative imaging technology, intraoperative localization of such disease has remained a formidable challenge with negative laparotomy rates reported as high as 30\% for patients with ileal and pancreatic endocrine tumors [686,691]. The intraoperative application of ultrasound, transduodenal illumination, and duodenotomy has been advocated by some [692] to pinpoint GEP neuroendocrine tumors particularly of the duodenum and pancreatic head region, a location historically known as the "gastrinoma triangle."

Another approach to intraoperative localization of GEP neuroendocrine tumors is use of the gamma detection probe for radioguided surgery. Radiopharmaceutical agents utilized for intraoperative detection of GEP neuroendocrine tumors include ${ }^{111}$ In-DTPA-D-Phe ${ }^{1}$-octreotide [693-695], 125I-Tyr ${ }^{3}$-octreotide [696,697], 123IMIBG [690], and 99mTc-EDDA/HYNIC octreotate [698]. However, the use of ${ }^{111}$ In-DTPA-D-Phe ${ }^{1}$-octreotide has become far more prevalent for several reasons. First, the considerably shorter physical half-life of ${ }^{111}$ In (approximately 68 hours) as compared to ${ }^{125}$ I (approximately 60 days) make ${ }^{111}$ In more desirable secondary to the importance of radiation safety issues related to the storage and disposal of radioactive materials [49]. Second, the predominant hepatobiliary excretion of ${ }^{125} \mathrm{I}_{-} \mathrm{Tyr}^{3}$-octreotide leads to a considerable amount of intestinal activity, reducing the reliability of somatostatin receptor imaging [688]. Third, the intestinal clearance of ${ }^{111}$ In-DTPA-DPhe ${ }^{1}$-octreotide can be overcome by the administration of laxatives. Lastly, the intraoperative tumor to background ratio is improved with most cases, demonstrating a ratio 
greater than 4:1 [694]. Despite these relative advantages and disadvantages, ${ }^{111} \mathrm{In},{ }^{125} \mathrm{I},{ }^{123} \mathrm{I}$ and $99 \mathrm{mTc}$ radiopharmaceutical agents have all been utilized to detect primary, recurrent, and metastatic lesions from multiple GEP neuroendocrine tumors, including gastrinomas, carcinoids, and insulinomas.

The standard technique for radioguided surgery of GEP neuroendocrine tumors involves the intravenous injection of ${ }^{111} \mathrm{In}$-DTPA-D-Phe ${ }^{1}$-octreotide at dosage range of 3.0 to $6.0 \mathrm{mCi}(110$ to $220 \mathrm{MBq})[684,687,694]$ with subsequent 4-hour and 24-hour whole body scintigraphy and 24-hour SPECT imaging. Surgical exploration is performed approximately 48 to 72 hours post-injection and is preceded by a bowel preparation to minimize the background radiation from physiologic bowel excretion of ${ }^{111}$ In-DTPA-D-Phe ${ }^{1}$-octreotide. A higher energy gamma detection probe (with a gamma photon energy range at or above the $247 \mathrm{keV}$ photopeak of ${ }^{111} \mathrm{In}$ ) is required. Once an area of high radioactivity is located, the gamma detection probe is held stationary and 10-second counts are obtained. A tumor to background ratio of at least 1.5 or greater is required for confirmation of the tissue localization [687]. If ${ }^{125} \mathrm{I}^{-\mathrm{Tyr}^{3}}$-octreotide is utilized instead of ${ }^{111}$ In-DTPA-D-Phe ${ }^{1}$-octreotide, the radiopharmaceutical agent is given the day of the procedure with Lugol's solution (a solution containing 5\% iodine $\left[\mathrm{I}_{2}\right]$ and $10 \%$ potassium iodide $[\mathrm{KI}]$ in $85 \%$ distilled water with a total iodine content of $130 \mathrm{mg} / \mathrm{mL}$ ) administered three days immediately before the planned surgery to prevent excessive thyroidal uptake of unbound 125I [697].

As reported by Adams and Baum [684], the use of the gamma detection probe with ${ }^{111}$ In-DTPA-D-Phe ${ }^{1}$-octreotide has increased the intraoperative detection of GEP neuroendocrine tumors, thus identifying 57\% more lesions than with conventional surgical palpation. In addition, the intraoperative gamma detection probe was able to identify small tumors (even down to less than 5 $\mathrm{mm}$ in size) more efficiently than somatostatin receptor scintigraphy (greater than $90 \%$ versus $68 \%$ to $77 \%$, respectively) [684]. As a result of this improved detection rate, cytoreductive radioguided surgery for GEP neuroendocrine tumors has resulted in improved long-term outcomes for patients with recurrent disease and/or metastatic disease $[685,699]$. In recurrent tumor surgery, much of the operative field is obscured with scar tissue prohibiting accurate localization with palpation. Radioguided surgery can distinguish tumor from scar tissue, thereby significantly aiding in the surgical dissection and extirpation of all sites of tumor $[695,696]$. As a result, radioguided surgery for detection of with ${ }^{111}$ In-DTPA-DPhe ${ }^{1}$-octreotide with the gamma detection probe has proven to be a useful adjunct in the management of GEP neuroendocrine tumors.
In a recent report by Hubalewska-Dydejczyk et al [698], they describe the use of $99 \mathrm{mTc}$-EDDA/HYNIC octreotate for radioguided surgery in patients with suspected GEP neuroendocrine tumors who had negative results on other preoperative imaging tests. Based on an $18.9 \mathrm{mCi}(700$ $\mathrm{MBq}$ ) dose of $99 \mathrm{mTc}$-EDDA/HYNIC octreotate intravenously injected 24 hours prior to surgery, radioguided detection of this new somatostatin analogue with the gamma detection probe was successful in identifying both carcinoid tumors and islet cell tumors within the study group.

Recently, ${ }^{68} \mathrm{Ga}$ has been labeled to octreotide by way of DOTA (1,4,7,10-tetraazacyclododecane-1,4,7,10-tetraacetic acid), a macrocyclic chelator, for the creation of ${ }^{68} \mathrm{Ga}$-DOTA-Tyr ${ }^{3}$-octreotide $[687,700]$ for the preoperative scintigraphic detection of GEP neuroendocrine tumors. The radionuclide ${ }^{68} \mathrm{Ga}$ has a physical half-life of approximately 68 minutes and decays by $89 \%$ through positron emission of a maximum energy of $1.92 \mathrm{MeV}$ (making it ideal for PET imaging) and by $11 \%$ through orbital electron capture. ${ }^{68} \mathrm{Ga}$-DOTA-Tyr ${ }^{3}$-octreotide has recently been described as a potentially highly desirable radiopharmaceutical agent for radioguided surgery for GEP neuroendocrine tumors [687]. Nevertheless, to date, no clinical studies involving actual radioguided surgical procedures for GEP neuroendocrine tumors have been published to substantiate this claim for ${ }^{68} \mathrm{Ga}$-DOTA-Tyr ${ }^{3}$ octreotide.

Most recently, ${ }^{18} \mathrm{~F}-\mathrm{L}-$ dihydroxyphenylalanine $\quad{ }^{18} \mathrm{~F}-\mathrm{L}-$ DOPA) was utilized for combined preoperative PET/CT imaging and radioguided surgery with the gamma detection probe for the detection of multiple site of metastatic GEP neuroendocrine tumor in a single patient with ${ }^{111}$ InDTPA-octreotide negative disease [701]. However, more time will be needed to thoroughly assess whether this ${ }^{18} \mathrm{~F}$ based technology will become clinically relevant in the surgical management of metastatic GEP neuroendocrine tumor.

\section{Bronchial carcinoids}

Only two case reports exist in the literature describing radioguided surgery with ${ }^{111}$ In pentetreotide for bronchial carcinoids. In 1997 Mansi et al [702] reported on a 28 year old male patient with Cushing's syndrome from an adrenocorticotrophin (ATCH)-secreting bronchial carcinoid who, four days after receiving a $3 \mathrm{mCi}(111 \mathrm{MBq})$ intravenous injection of ${ }^{111}$ In-DTPA-D-Phe ${ }^{1}$-octreotide, underwent radioguided surgery with the assistance of a gamma detection probe and which allowed for accurate localization and resection of a $1.8 \mathrm{~cm}$ tumor in the right lower lobe with in vivo tumor to normal surrounding tissue count ratios of $6: 1$ and ex vivo tumor to normal surrounding tissue count ratios of 18:1. Similarly, in 2005, Gross- 
rubatscher et al [703] reported on a 28 year old female patient with Cushing's syndrome from an adrenocorticotrophin (ATCH)-secreting bronchial carcinoid who, two days after receiving a $4 \mathrm{mCi}(148 \mathrm{MBq})$ intravenous injection of ${ }^{111}$ In-DTPA-D-Phe ${ }^{1}$-octreotide, underwent radioguided thoracoscopic surgery with the assistance of a gamma detection probe and which allowed for accurate localization and resection of multiple metastatic lymph nodes within the upper mediastinal region.

\section{Neuroblastoma}

Only very limited data are available on the use of the gamma detection probe during radioguided surgery for neuroblastoma [684,704-707]. This was first reported in 1995 by Martinez et al [704] in six pediatric patients with stage II or IV neuroblastoma using an intravenous injection of approximately 0.03 to $0.2 \mathrm{mCi}(1.1$ to $7.4 \mathrm{MBq}$ ) of ${ }^{125} \mathrm{I}_{-} \mathrm{Tyr}^{3}$-octreotide given 1.8 to 7.5 hours prior to surgery. The gamma detection probe found 17 sites of ${ }^{125} \mathrm{I}_{-} \mathrm{Tyr}^{3}{ }_{-}$ octreotide binding, and of which 15 sites contained neuroblastoma. Five of these 15 sites of neuroblastoma were occult and were not detected by standard intraoperative palpation and visualization. Next, in 1997, Heij et al [705] reported on five pediatric patients with recurrent abdominal neuroblastoma that were intravenously injected with $5 \mathrm{mCi}(185 \mathrm{MBq})$ of 123I-MIBG that was given 48 hours prior to surgery. The gamma detection probe correctly identified tumor seen on preoperative MIGB scan in all five patients. Thereafter, in 1998, Martelli et al [706] reported the largest series of 66 radioguided surgical procedures in 58 pediatric patients undergoing intraoperative localization of neuroblastoma with the gamma detection probe and utilizing either ${ }^{123}$ I-MIBG (mean dose of $5 \mathrm{mCi}$ or $185 \mathrm{MBq}$ intravenously injected 24 to 48 hours before surgery) or ${ }^{125}$ I-MIBG (mean dose of $0.11 \mathrm{mCi}$ or $4 \mathrm{MBq}$ intravenously injected three to five days before surgery). A tumor to background ratio that exceeded 2:1 was considered positive for target localization, and in $65 \%$ of the cases, the gamma detection probe was considered helpful. The gamma detection probe permitted detection of small, nonpalpable tumors in sites that were difficult to access surgically as well as aided in more accurately defining tumor margins or areas of tumor extension. The sensitivities of the 123 I-MIBG and ${ }^{125}$ IMIBG were similar, at $91 \%$ and $92 \%$ respectively. However, the specificity of 125 I-MIBG was significantly higher than ${ }^{123}$ I-MIBG ( $85 \%$ as compared to $55 \%$, respectively). Comparable results for ${ }^{123}$ I-MIBG and ${ }^{125}$ I-MIBG in neuroblastoma patients were reported by Adams and Baum [684]. In addition, Adams and Baum [684] attempted intraoperative neuroblastoma localization with ${ }^{111}$ InDTPA-D-Phe ${ }^{1}$-octreotide. However, they found the urinary excretion of the radionuclide limited the use of the gamma probe in tissues adjacent to the kidneys and ureters.

\section{Pheochromocytoma}

MIBG scintigraphy, with a sensitivity and specificity as high as $88 \%$ and $96 \%$, respectively, is the technique of choice for the localization of pheochromocytoma [708]. Only very limited data is available on intraoperative localization of pheochromocytomas with iodine-based radiopharmaceutical MIBG agents, including 131I-MIBG [709], 125I-MIBG [710,711], and 123I-MIBG [463] and has confirmed the feasibility of this technique. The technique for preoperative administration of the iodine-based radiopharmaceutical MIBG agent varies based on the physical half-life of the agent, with Proye et al [710] reporting intravenously injecting $0.5 \mathrm{mCi}$ to $1.0 \mathrm{mCi}$ (18 MBq to 37 $\mathrm{MBq}$ ) of ${ }^{125}$ I-MIBG approximately 3 days before surgery with thyroid blockade and with Adams et al [463] reporting intravenously injecting $4.9 \mathrm{mCi}$ (180 MBq) of ${ }^{123} \mathrm{I}-$ MIBG approximately 4 to 5 hours before surgery without thyroid blockade. Tumor to background ratios exceeding $5: 1$ were achieved $[710,711]$. Detection of tumor foci less than or equal to one centimeter in size was demonstrated [711]. However, Adams et al [463] suggested that avid hepatic and biliary signals may overshadow potential intraoperative sites of pathologic MIGB uptake seen on whole body scanning, especially in the subdiaphragmatic/ paravertebral region, and may lead to low tumor to background ratios of 1.5:1.0. As a result, only very limited acceptance of this radioguided surgical technique has been given since these early published series.

\section{Adrenocortical carcinoma}

The use of the gamma detection probe in radioguided surgery for adrenocortical carcinoma is virtually nonexistent. Only one report exists in the literature that describes a single case of ${ }^{18} \mathrm{~F}$-FDG-directed surgery for adrenocortical carcinoma [45]. In this report, ${ }^{18}$ F-FDG-directed surgery was instrumental in locating difficult to access metastatic lymph nodes at the time of a combined sternotomy and laparatomy.

\section{Sarcoma}

The application of the gamma detection probe for the surgical management of sarcoma has been extremely limited. The vast majority of sarcoma histologic subtypes metastasize only hematologically. Nevertheless, a few sarcoma histologic subtypes have a propensity for lymphatic dissemination, such as rhabdomyosarcoma, epithelioid sarcoma, clear cell sarcoma, and synovial sarcoma [712]. In this regard, radioguided SLN biopsy has been limitedly investigated in some of these select sarcoma histologic subtypes [713-722]. These reports are limited to a few case reports [714,716,717,719] and to a few small series [713,715,718,720-722]. Most frequently, 99mTc colloidal human albumin, $99 \mathrm{mTc}$ sulfur colloid, and $99 \mathrm{mTc}$ rhenium sulfur colloid have been utilized. However, the timing of injection, site of injection, and total dosage range for the 
radiocolloid have generally not been well-characterized in these limited reports.

The largest series reported to date was recently published by Kayton et al [721]. In their report, they described 23 pediatric patients with sarcoma (9 rhabdomyosarcomas, 4 epithelioid sarcomas, 2 synovial sarcomas, 2 alveolar soft part sarcomas, 2 fibrosarcomas, 2 clear cell sarcomas, 1 Ewing sarcoma, and 1 angiomatoid fibrous histiocytoma) undergoing radioguided SLN biopsy using a dosage range of $0.05 \mathrm{mCi}(1.9 \mathrm{MBq})$ to $0.8 \mathrm{mCi}(29.6 \mathrm{MBq})$ of 99mTc sulfur colloid that was injected into one to four locations at the site of the primary tumor. A SLN was detected in all patients. A positive SLN was found in only one patient (4.3\%). Since the radioguided SLN biopsy was not accompanied by a confirmatory lymph node dissection, a false negative rate could not be determined.

\section{Brain tumors}

Radioguided surgical resection of brain tumors has been investigated in a very limited fashion [723-727]. In 2002, Vilela Filho and Carneiro Filho [723] first described use of 99mTc-MIBI, in a dose of $30 \mathrm{mCi}(1110 \mathrm{MBq})$, for gamma probe-assisted resection of a metastatic renal cell carcinoma to the right parietal lobe. In 2004, Kojima et al [724] reported on the use of 99mTc-MIBI, in an unknown dose, for gamma probe-assisted resection in 13 patients with either primary or recurrent astrocytomas or metastatic brain lesions. In 2005, Gay et al [725] reported on the use of ${ }^{111}$ In-(DTPA)-D-Phe1 pentetreotide, an analog of somatostatin, in a dose of $3 \mathrm{mCi}(111 \mathrm{MBq})$, for gamma probe-assisted resection in 10 patients with en plaque meningiomas. In 2006, Serrano et al [726] reported on the use of ${ }^{201} \mathrm{Tl}$, in a dose of $1.4 \mathrm{mCi}(50 \mathrm{MBq})$, for gamma probe-assisted resection of an astrocytoma of the right temporoparietal region. Finally, in 2007, Bhanot et al [727] reported on the use of ${ }^{99 \mathrm{~m} T \mathrm{Tc}-\mathrm{MIBI} \text {, in a dose of } 10}$ $\mathrm{mCi}(370 \mathrm{MBq})$, for gamma probe-assisted resection in 13 patients with supratentorial gliomas. However, at this time, other methods of intraoperative neurosurgical navigation, such as with magnetic resonance imaging and ultrasound, which do not utilize radioactive substances, remain the standard of care.

\section{Bone lesions}

The use of the gamma detection probe in radioguided surgery has been extensively described for radioguided biopsy and/or resection of benign bone lesions, such as osteoid osteomas [728-742] and enchondromas [743], as well as suspected bone metastases [743-755]. The application of gamma detection probe technology for radioguided biopsy and/or resection of bone lesions in the early 1980 s $[728,743]$ truly represents some of the early forward thinking that contributed to the later development of the modern era of radioguided surgery (Table 1).
The technique radioguided biopsy and/or resection of bone lesions utilized the same class of radiopharmaceutical agents ( ${ }^{99 \mathrm{~m} T c}$ diphosphonates) and the same dosing range as is utilized for diagnostic bone scanning. The most common ${ }^{99 \mathrm{~m} T \mathrm{~T}}$ diphosphonates utilized are $99 \mathrm{mTc}$ MDP, 99mTc HMDP, and 99mTc HDP. These radiopharmaceutical agents are administered by a peripheral intravenous injection in a dosing range of approximately $15 \mathrm{mCi}(555$ $\mathrm{MBq})$ to $30 \mathrm{mCi}(1,110 \mathrm{MBq})$ at a time approximately 2 to 12 hours prior to the planned surgical procedure. Reasonable success has been reported with guiding diagnostic biopsy of such lesions as well as guiding the surgeon for complete surgical resection of such lesions. Nevertheless, this technique, as it relates to bone lesions, has not become a part of the mainstream clinical practice for the biopsy and/or resection of bone lesions.

\section{Lymphoma}

The application of intraoperative gamma probe-directed biopsy of suspicious lesions for confirming the diagnosis of lymphoma has been very limited $[43,45,746,755,756]$. Robinson et al [747] described using 99mTc HMDP for intraoperative gamma probe-directed biopsy of two rib lesions representing lymphoma. Schattner et al [756] described using ${ }^{67} \mathrm{Ga}$ for intraoperative gamma probedirected biopsy of increased activity within the left paraspinal and psoas muscles seen on whole body gallium scan and single-photon emission computed tomography (SPECT) for confirming the diagnosis of lymphoma. Burdine et al [757] described using ${ }^{99 \mathrm{~m} T \mathrm{Tc}}$ sulfur colloid for intraoperative gamma probe-directed biopsy of small, nonpalpable, non-visible, suspicious pulmonary nodules during video-assisted thoracoscopic surgery in two patients with a history of lymphoma. Most recently, Gulec et al $[43,45]$ have described using ${ }^{18}$ F-FDG for intraoperative gamma probe-directed biopsy of nonpalpable suspicious lymph nodes seen on diagnostic whole body PET scan for confirming the diagnosis of lymphoma.

\section{Radioguided monitoring of isolated limb perfusion}

The utility of a gamma detection probe system for intraoperative radioguided monitoring of systemic leakage during isolated limb perfusion surgery for both melanoma and sarcoma has been previously investigated [758-767]. The basic principles of this technique were first described in 1961 by Stehlin et al [758] using a single, large, overhead-mounted scintillation detector that displayed continuous tracing results, in counts per minute, on a rectilinear recorder for detecting ${ }^{131}$ I-labeled human serum albumin. The principles of this technique using a more modern handheld gamma detection probe system within the operating room were later described in 1989 by Sardi et al [759] at The Ohio State University. This system by Sardi et al [759] consisted on two handheld gamma probes, with one positioned over the precordial area and 
one positioned over the distal aspect of the thigh. Each patient received $0.8 \mathrm{mCi}(29.6 \mathrm{MBq})$ of $99 \mathrm{mTc}$ pentetate through the perfusate circulation. The percentage of ${ }^{99 \mathrm{~m} T c}$ pentetate leakage was calculated by a simultaneous reading of the two gamma detection probes at one-minute intervals. When compared to a method of intermittent simultaneous blood sampling from the perfusate and systemic circulations at 15 minutes intervals, essentially identical percentage of leakage was detected by both methods. However, the minute by minute monitoring of the two handheld gamma probe system allowed for a more instantaneous and real-time indication of any fluctuations in the percentage of leakage than did the intermittent (every 15-minute) blood sampling from the perfusate and systemic circulations.

Since that time, multiple variations of this technique have been described [760-767]. Manner et al [760] have advocated the use of a two-probe system (precordial and thigh) with $0.50 \mathrm{mCi}(18.5 \mathrm{MBq})$ of ${ }^{111}$ In-labeled red blood cells injected into the perfusate circulation. Sprenger et al [761] have advocated the use of a three-probe system (precordial, thigh, and perfusate circuit) with low-dose (i.e., $0.004 \mathrm{mCi}$ or $0.15 \mathrm{MBq}$ ) of ${ }^{111}$ In-labeled red blood cells injected into the systemic circulation (to establish a minimum baseline reference activity within the systemic circulation) and a subsequent high-dose (i.e., $0.32 \mathrm{mCi}$ or 12 $\mathrm{MBq})$ of ${ }^{111}$ In-labeled red blood cells injected into the perfusate circulation. Sandrock et al [763] have advocated the use of a precordial one-probe system with 99mTclabeled red blood cells $(0.4 \mathrm{mCi}$ or $15 \mathrm{MBq})$ injected into the perfusate circulation. Barker et al [762] have advocated the use of a precordial one-probe system with a combination radionuclide design consisting of a low-dose (i.e., $0.02 \mathrm{mCi}$ or $0.74 \mathrm{MBq}$ ) of ${ }^{131}$ I-labeled human serum albumin injected into the systemic circulation and a subsequent 10 -fold higher dose (i.e., $0.20 \mathrm{mCi}$ or $7.4 \mathrm{MBq}$ ) of ${ }^{131}$ I-labeled human serum albumin injected into the perfusate circulation. Similarly, Daryanani et al [764] and Van Ginkel et al [765] have advocated the use of a precordial one-probe system with a combination radionuclide design consisting of a low-dose (i.e., $0.014 \mathrm{mCi}$ or 0.5 $\mathrm{MBq})$ of ${ }^{131} \mathrm{I}$-labeled human serum albumin and $0.3 \mathrm{mCi}$ (10 MBq) of $99 \mathrm{~m}$ Tc-labeled human serum albumin injected into the systemic circulation and a subsequent 10 -fold higher dose (i.e., $0.14 \mathrm{mCi}$ or $5 \mathrm{MBq}$ ) of ${ }^{131} \mathrm{I}-$ labeled human serum albumin injected into the perfusate circulation. Finally, Casara et al [766,767] have advocated a precordial one-probe system with $0.014 \mathrm{mCi} / \mathrm{kg}(0.5$ $\mathrm{MBq} / \mathrm{kg}$ ) of ${ }^{99 \mathrm{mTc}} \mathrm{T}-\mathrm{labeled}$ human serum albumin injected into the perfusate circulation at the time of the isolated limb perfusion surgery and in conjunction with prior simulation testing with $0.0014 \mathrm{mCi} / \mathrm{kg}(0.05 \mathrm{MBq} / \mathrm{kg})$ of 99mTc-labeled human serum albumin injected into the systemic circulation of the patient at a time 48 to 72 hours prior to the isolated limb perfusion surgery. Despite variations in these techniques, all have verified the potential utility of the gamma detection probe for intraoperative radioguided monitoring of systemic leakage during isolated limb perfusion surgery.

\section{Conclusion}

Radioguided surgery using gamma detection probe technology has tremendously expanded and has evolved into what is now considered an established discipline within the practice of surgery. It has been investigated in and applied to almost every imaginable neoplastic disease entity that is surgically managed, and it has revolutionized the surgical management of breast cancer, melanoma, colorectal cancer, and parathyroid disease. The impact of radioguided surgery on the surgical management of cancer patients includes providing vital and real-time information to the surgeon regarding the location and extent of disease, as well as regarding the assessment of surgical resection margins. Additionally, it has allowed the surgeon to minimize the surgical invasiveness of many diagnostic and therapeutic procedures, while still maintaining maximum benefit to the cancer patient. As we move forward through the $21^{\text {st }}$ century, we hope to continue to build upon our current technological platform of radioguided surgery and further integrate advanced medical imaging within the operative arena in order to supplement intraoperative gamma detection probe technology $[16,768]$.

\section{Competing interests}

The authors declare that they have no competing interests.

\section{Authors' contributions}

The initial planning and development of the concept and content of the review manuscript was undertaken by SPP and EWM. The individual sections comprising the review manuscript were written by SPP, RLN, CMM, and DMO. SPP assumed the supervising editorial role for all the authors and was responsible for editing and organizing all the individual sections into the review manuscript. EWM was the senior physician overseeing the entire project. RLN, CMM, DMO, GHH, NCH, DAM, MVK, and EWM all played a critical role and had a substantial contribution to the revising and editing process of the review manuscript. All of the authors have read and approved the final version of the review manuscript.

\section{Acknowledgements}

The authors would like to dedicate this current review manuscript in the memory of Dr. Marlin O. Thurston, Professor Emeritus of Electrical Engineering at The Ohio State University, who played an instrumental role in the development of the field of radioguided surgery and who passed away on September 18, 2007. 


\section{References}

I. Selverstone B, Sweet WH, Robinson CV: The clinical use of radioactive phosphorus in the surgery of brain tumors. Ann Surg 1949, I 30:643-65I.

2. Geiger H, Müller W: Elektronenzählrohr zur messung schwächster aktivitäten. Naturwissenschaften 1928, 16:617-618.

3. Harris CC, Bigelow RR, Francis JE, Kelly GG, Bell PR: A Csi(Ti)crystal surgical scintillation probe. Nucleonics 1956, I4: I02-108.

4. Woolfenden JM, Barber HB: Radiation detector probes for tumor localization using tumor-seeking radioactive tracers. AJR Am J Roentgenol 1989, I 53:35-39.

5. Schneebaum S, Even-Sapir E, Cohen M, Shacham-Lehrman H, Gat A, Brazovsky E, Livshitz G, Stadler J, Skornick Y: Clinical applications of gamma-detection probes-radioguided surgery. Eur J Nucl Med 1999, 26:S26-S35.

6. Barber HB, Barrett HH, Hickernell TS, Kwo DP, Woolfenden JM, Entine G, Ortale Baccash C: Comparison of Nal(TI), CdTe, and Hgl2 surgical probes: physical characterization. Med Phys |99|, | 8:373-381.

7. Kwo DP, Barber HB, Barrett HH, Hickernell TS, Woolfenden JM: Comparison of $\mathrm{Nal}(\mathrm{TI}), \mathrm{CdTe}$, and $\mathrm{HgI} 2$ surgical probes: effect of scatter compensation on probe performance. Med Phys 1991, I 8:382-389.

8. Thurston MO: Development of the gamma-detecting probe for radioimmunoguided surgery. In Radioimmunoguided Surgery (RIGS) in the Detection and Treatment of Colorectal Cancer Ist edition. Edited by: Martin EW. Austin: R.G. Landes Company; 1994:41-65.

9. Tiourina T, Arends B, Huysmans D, Rutten H, Lemaire B, Muller S: Evaluation of surgical gamma probes for radioguided sentinel node localisation. Eur J Nucl Med 1998, 25: I224-I23I.

10. Hoffman EJ, Tornai MP, Janecek M, Patt BE, Iwanczyk JS: Intraoperative probes and imaging probes. Eur J Nucl Med 1999, 26:913-35.

II. Zanzonico P, Heller S: The intraoperative gamma probe: basic principles and choices available. Semin Nucl Med 2000, 30:33-48.

12. Ricard $M$ : Intraoperative detection of radiolabeled compounds using a hand held gamma probe. Nucl Instrum Meth Phys Res A 200I, 458:26-33.

13. Zanzonico $P$ : The intraoperative gamma probe: design, operation, and safety. In Sentinel Lymph Node Biopsy Ist edition. Edited by: Cody HS. London: Informa Health Care; 2002:45-68.

14. Wengenmair H, Kopp J: Gamma probes for sentinel lymph node localization: quailty criteria, minimal requirements and quality of commercially available systems. 2005 [http:// www.sln-kompetenzzentrum.de/gammaprobes.pdf].

I5. Mariani G, Vaiano A, Nibale O, Rubello D: Is the "ideal" gammaprobe for intraoperative radioguided surgery conceivable? J Nucl Med 2005, 46:388-90.

16. Moffat FL Jr: Targeting gold at the end of the rainbow: surgical gamma probes in the 2 Ist century. J Surg Oncol 2007, 96:286-289.

17. Sarikaya I, Sarikaya A, Reba RC: Gamma probes and their use in tumor detection in colorectal cancer. Int Semin Surg Oncol 2008, 5:25.

18. Daghighian F, Mazziotta JC, Hoffman EJ, Shenderov P, Eshaghian B, Siegel S, Phelps ME: Intraoperative beta probe: a device for detecting tissue labeled with positron or electron emitting isotopes during surgery. Med Phys 1994, 2 I: I 53-157.

19. Raylman RR, Wahl RL: A fiber-optically coupled positron-sensitive surgical probe. I Nucl Med 1994, 35:909-9/3.

20. Raylman RR, Wahl RL: Evaluation of ion-implanted-silicon detectors for use in intraoperative positron-sensitive probes. Med Phys 1996, 23:1889-1895.

21. Raylman RR: A solid-state intraoperative beta probe system. IEEE Trans Nucl Sci 2000, 47:1696-I703

22. Yamamoto S, Matsumoto K, Sakamoto S, Tarutani K, Minato K, Senda $M$ : An intra-operative positron probe with background rejection capability for FDG-guided surgery. Ann Nucl Med 2005, 19:23-28.

23. Yamamoto S, Higashi T, Matsumoto K, Senda M: Development of a positron-imaging detector with background rejection capability. Ann Nucl Med 2006, 20:655-662.

24. Gamma Finder ${ }^{\circledR}$ II, W.O.M. World of Medicine AG [http:// www.gammafinder.com/]

25. Bluetooth ${ }^{\circledR}$ Gamma Detection Probe, Neoprobe Corporation.
Model\%201100\%20and\%201101\%20Bluetooth\%20Manual English.pd f and http://salesandmarketingnetwork.com/ news release.php? ID $=2014104$

26. Hinkle GH, Laven DL: Radionucleotides. In Radioimmunoguided Surgery (RIGS) in the Detection and Treatment of Colorectal Cancer I st edition. Edited by: Martin EW. Austin: R.G. Landes Company; 1994:29-39.

27. International Atomic Energy Agency (IAEA) Nuclear Data Services [http://www-nds.iaea.org/xgamma standards/]

28. Sergides IG, Austin RC, Winslet MC: Radioimmunodetection: technical problems and methods of improvement. Eur J Surg Oncol 1999, 25:529-539.

29. Strong VE, Humm J, Russo P, Jungbluth A, Wong WD, Daghighian F, Old L, Fong Y, Larson SM: A novel method to localize antibodytargeted cancer deposits intraoperatively using handheld PET beta and gamma probes. Surg Endosc 2008, 22:386-391.

30. Nieroda CA, Milenic DE, Colcher D, Schlom J: Monoclonal Antibodies for Use in Radioimmunoguided Surgery (RIGS). In Radioimmunoguided Surgery (RIGS) in the Detection and Treatment of Colorectal Cancer Ist edition. Edited by: Martin EW. Austin: R.G. Landes Company; 1994:7-27.

31. Mitchell ACG, Juliano JO, Creager CB, Kocher CW: Disintegration of I'24 and I'23. Phys Rev 1959, I | 3:628-633.

32. Pentlow KS, Graham MC, Lambrecht RM, Daghighian F, Bacharach SL, Bendriem B, Finn RD, Jordan K, Kalaigian H, Karp JS, Robeson WR, Larson SM: Quantitative imaging of iodine-I 24 with PET. J Nucl Med 1996, 37:1557-I562.

33. Lawson RS: An Introduction to Radioactivity. [http:www.e-radiography.net/articles/Introduc tion\%20to\%20Radioactivity.pdf].

34. Rault E, Vandenberghe S, Van Holen R, De Beenhouwer J, Staelens S, Lemahieu I: Comparison of image quality of different iodine isotopes (I-I23, I-I24, and I-I3I). Cancer Biother Radiopharm 2007, 22:423-430.

35. Desai D, Arnold M, Saha S, Hinkle G, Soble D, Frye J, DePalatis L, Mantil J, Satter M, Martin E: Intraoperative gamma detection of FDG distribution in colorectal cancer. Clin Positron Imaging 1999, 2:325.

36. Desai DC, Arnold M, Saha S, Hinkle G, Soble D, Fry J, DePalatis LR, Mantil J, Satter M, Martin EW: Correlative whole-body FDG-PET and intraoperative gamma detection of FDG distribution in colorectal cancer. Clin Positron Imaging 2000, 3:189-196.

37. Zervos EE, Desai DC, DePalatis LR, Soble D, Martin EW: I 8Flabeled fluorodeoxyglucose positron emission tomographyguided surgery for recurrent colorectal cancer: a feasibility study. J Surg Res 200I, 97:9-13.

38. Essner R, Hsueh EC, Haigh PI, Glass EC, Huynh Y, Daghighian F: Application of an [( 18$) F]$ fluorodeoxyglucose-sensitive probe for the intraoperative detection of malignancy. I Surg Res 2001, 96:120-126.

39. Essner R, Daghighian F, Giuliano AE: Advances in FDG PET probes in surgical oncology. Cancer J 2002, 8:100-108.

40. Carrera D, Fernandez A, Estrada J, Martin-Comin J, Gamez C: [Detection of occult malignant melanoma by I 8F-FDG PETCT and gamma probe]. Rev Esp Med Nucl 2005, 24:4I0-4I3. [Spanish]

4I. Franc BL, Mari C, Johnson D, Leong SP: The role of a positronand high-energy gamma photon probe in intraoperative localization of recurrent melanoma. Clin Nucl Med 2005, 30:787-79l.

42. Kraeber-Bodéré F, Cariou B, Curtet C, Bridji B, Rousseau C, Dravet F, Charbonnel B, Carnaille B, Le Néel JC, Mirallié E: Feasibility and benefit of fluorine I 8-fluoro-2-deoxyglucose-guided surgery in the management of radioiodine-negative differentiated thyroid carcinoma metastases. Surgery 2005, I 38: I I 76- I I82.

43. Gulec SA, Daghighian F, Essner R: PET-Probe: Evaluation of Technical Performance and Clinical Utility of a Handheld High-Energy Gamma Probe in Oncologic Surgery. Ann Surg Oncol 2006 in press.

44. Meller B, Sommer K, Gerl J, von Hof K, Surowiec A, Richter E, Wollenberg $B$, Baehre $M$ : High energy probe for detecting lymph node metastases with I8F-FDG in patients with head and neck cancer. Nuklearmedizin 2006, 45: I53-I59.

45. Gulec SA, Hoenie E, Hostetter R, Schwartzentruber D: PET probeguided surgery: applications and clinical protocol. World J Surg Oncol 2007, 5:65. 
46. Gulec SA: PET probe-guided surgery. I Surg Oncol 2007 96:353-357.

47. Piert M, Burian M, Meisetschlager G, Stein HJ, Ziegler S, Nahrig J, Picchio M, Buck A, Siewert JR, Schwaiger M: Positron detection for the intraoperative localisation of cancer deposits. Eur J Nucl Med Mol Imaging 2007, 34: I534-I544.

48. Piert M, Carey J, Clinthorne N: Probe-guided localization of cancer deposits using $[(\mathbf{1 8}) \mathrm{F}]$ fluorodeoxyglucose. Q J Nucl Med Mol Imaging 2008, 52:37-49.

49. Sun D, Bloomston M, Hinkle G, Al-Saif $\mathrm{OH}$, Hall NC, Povoski SP, Arnold MW, Martin EW: Radioimmunoguided surgery (RIGS), PET/CT image-guided surgery, and fluorescence imageguided surgery: past, present, and future. J Surg Oncol 2007, 96:297-308.

50. Sarikaya I, Povoski SP, AI-Saif OH, Kocak E, Bloomston M, Marsh S, Cao Z, Murrey DA, Zhang J, Hall NC, Knopp MV, Martin EW: Combined use of preoperative I8F FDG-PET imaging and intraoperative gamma probe detection for accurate assessment of tumor recurrence in patients with colorectal cancer. World J Surg Oncol 2007, 5:80.

5I. Hall NC, Povoski SP, Murrey DA, Knopp MV, Martin EW: Combined approach of perioperative 18 F-FDG PET/CT imaging and intraoperative 18 F-FDG handheld gamma probe detection for tumor localization and verification of complete tumor resection in breast cancer. World J Surg Oncol 2007, 5:143.

52. Povoski SP, Hall NC, Martin EW, Walker MJ: Multimodality approach of perioperative 18 F-FDG PET/CT imaging, intraoperative 18 F-FDG handheld gamma probe detection, and intraoperative ultrasound for tumor localization and verification of resection of all sites of hypermetabolic activity in a case of occult recurrent metastatic melanoma. World J Surg Oncol 2008, 6: I.

53. Cohn DE, Hall NC, Povoski SP, Seamon LG, Farrar WB, Martin EW Jr: Novel perioperative imaging with I8F-FDG PET/CT and intraoperative $18 F-F D G$ detection using a handheld gamma probe in recurrent ovarian cancer. Gynecol Oncol 2008 II 0:152-I57.

54. Moffatt-Bruce SD, Povoski SP, Sharif S, Hall NC, Ross P Jr, Johnson MA, Martin EW Jr: A novel approach to positron emission tomography in lung cancer. Ann Thorac Surg 2008, 86: I355-I357.

55. Snyder SE, Kilbourne MR: Chemistry of fluorine-18 radiopharmaceuticals. In Handbook of Raiopharmaceuticals: Radiochemistry and Applications Ist edition. Edited by: Welch MJ, Redvanly CS. Hoboken John Wiley and Sons, Ltd; 2003:195-228.

56. Fowler JS, Ido $T$ : Design and synthesis of 2-deoxy-2-[18 F] fluoro-D-glucose (18 FDG). In Handbook of Raiopharmaceuticals: Radiochemistry and Applications I st edition. Edited by: Welch MJ, Redvanly CS. Hoboken: John Wiley and Sons, Ltd; 2003:307-322.

57. Hinkle GH, Houchens DP, Sampsel J, Schneebaum S: The RIGS System Using Animal Models. In Radioimmunoguided Surgery (RIGS) in the Detection and Treatment of Colorectal Cancer I st edition. Edited by: Martin EW. Austin: R.G. Landes Company; 1994:67-80.

58. Martin EW Jr, Barnes JA, Thurston MO: Introduction: The RIGS Concept. In Radioimmunoguided Surgery (RIGS) in the Detection and Treatment of Colorectal Cancer Ist edition. Edited by: Martin EW. Austin: R.G. Landes Company; 1994: I-6.

59. Martin EW Jr, Thurston MO: The use of monoclonal antibodies (MAbs) and the development of an intraoperative hand-held probe for cancer detection. Cancer Invest 1996, 14:560-57I.

60. Köhler G, Milstein C: Continuous cultures of fused cells secreting antibody of predefined specificity. Nature 1975 256:495-497.

61. Martin EW Jr, Thurston MO: Intraoperative radioimmunodetection. Semin Surg Oncol 1998, 15:205-208.

62. Johnson VG, Schlom J, Paterson AJ, Bennett J, Magnani JL, Colcher D. Analysis of a human tumor-associated glycoprotein (TAG72) identified by monoclonal antibody B72.3. Cancer Res 1986, 46:850-857.

63. Sheer DG, Schlom J, Cooper HL: Purification and composition of the human tumor-associated glycoprotein (TAG-72) defined by monoclonal antibodies CC49 and B72.3. Cancer Res 1988 , 48:68II-68I8

64. Colcher D, Milenic D, Roselli M, Raubitschek A, Yarranton G, King D, Adair J. Whittle N, Bodmer M, Schlom J: Characterization and biodistribution of recombinant and recombinant/chimeric constructs of monoclonal antibody B72.3. Cancer Res 1989 , 49:1738-1745.

65. Stramignoni $D$, Bowen $R$, Atkinson $B F$, Schlom J: Differential reactivity of monoclonal antibodies with human colon adenocarcinomas and adenomas. Int J Cancer 1983, 31:543-552.

66. Ohuchi N, Simpson JF, Colcher D, Schlom J: Complementation of anti-CEA and anti-TAG-72 monoclonal antibodies in reactivity to human gastric adenocarcinomas. Int J Cancer 1987, 40:726-733.

67. Thor A, Gorstein F, Ohuchi N, Szpak CA, Johnston WW, Schlom J: Tumor-associated glycoprotein (TAG-72) in ovarian carcinomas defined by monoclonal antibody B72.3. I Natl Cancer Inst 1986, 76:995-1006.

68. Nuti M, Teramoto YA, Mariani-Costantini R, Hand PH, Colcher D, Schlom J: A monoclonal antibody (B72.3) defines patterns of distribution of a novel tumor-associated antigen in human mammary carcinoma cell populations. Int J Cancer 1982, 29:539-545.

69. Thor A, Ohuchi N, Szpak CA, Johnston WW, Schlom J: Distribution of oncofetal antigen tumor-associated glycoprotein-72 defined by monoclonal antibody B72.3. Cancer Res 1986, 46:3II8-3I24.

70. Colcher D, Hand PH, Nuti M, Schlom J: A spectrum of monoclonal antibodies reactive with human mammary tumor cells. Proc Natl Acad Sci USA I98I, 78:3 199-3203.

7I. Thor A, Viglione MJ, Muraro R, Ohuchi N, Schlom J, Gorstein F: Monoclonal antibody $B 72.3$ reactivity with human endometrium: a study of normal and malignant tissues. Int J Gynecol Pathol 1987, 6:235-247.

72. Muraro R, Kuroki M, Wunderlich D, Poole DJ, Colcher D, Thor A, Greiner JW, Simpson JF, Molinolo A, Noguchi P, Schlom J: Generation and characterization of B72.3 second generation monoclonal antibodies reactive with the tumor-associated glycoprotein 72 antigen. Cancer Res 1988, 48:4588-4596.

73. Colcher D, Minelli MF, Roselli M, Muraro R, Simpson-Milenic D, Schlom J: Radioimmunolocalization of human carcinoma xenografts with B72.3 second generation monoclonal antibodies. Cancer Res 1988, 48:4597-4603.

74. Arnold MW, Schneebaum S, Berens A, Petty L, Mojzisik C, Hinkle G, Martin EW Jr: Intraoperative detection of colorectal cancer with radioimmunoguided surgery and $\mathrm{CC} 49$, a second-generation monoclonal antibody. Ann Surg 1992, 216:627-632.

75. Arnold MW, Schneebaum S, Berens A, Mojzisik C, Hinkle G, Martin EW Jr: Radioimmunoguided surgery challenges traditional decision making in patients with primary colorectal cancer. Surgery 1992, I I 2:624-629.

76. Schroff RW, Foon KA, Beatty SM, Oldham RK, Morgan AC Jr: Human anti-murine immunoglobulin responses in patients receiving monoclonal antibody therapy. Cancer Res 1985, 45:879-885.

77. Colcher D, Milenic DE, Ferroni P, Carrasquillo JA, Reynolds JC, Roselli M, Larson M, Schlom J: In vivo fate of monoclonal antibody B72.3 in patients with colorectal cancer. J Nucl Med 1990, 3:II33-II 42

78. Mojzisik CM, Cook CH, Schneebaum S: Safety and Performance Factors in the Development and Clinical Use of the RIGS System. In Radioimmunoguided Surgery (RIGS) in the Detection and Treatment of Colorectal Cancer Ist edition. Edited by: Martin EW. Austin: R.G. Landes Company; 1994:8I-105.

79. Frödin JE, Lefvert AK, Mellstedt $H$ : The clinical significance of HAMA in patients treated with mouse monoclonal antibod. ies. Cell Biophys 1992, 21:153-165.

80. Gruber R, van Haarlem LJ, Warnaar SO, Holz E, Riethmüller G: The human antimouse immunoglobulin response and the antiidiotypic network have no influence on clinical outcome in patients with minimal residual colorectal cancer treated with monoclonal antibody COI7-IA. Cancer Res 2000, 60:1921-1926.

81. Kashmiri SV, Shu L, Padlan EA, Milenic DE, Schlom J, Hand PH: Generation, characterization, and in vivo studies of humanized anticarcinoma antibody CC49. Hybridoma 1995, I 4:46 I-473.

82. Xiao J, Horst S, Hinkle G, Cao X, Kocak E, Fang J, Young D, Khazael M, Agnese D, Sun D, Martin E Jr: Pharmacokinetics and clinical evaluation of |25|-radiolabeled humanized CC49 monoclonal antibody $(\mathrm{HuCC} 49 \mathrm{deltaC}(\mathrm{H}) 2)$ in recurrent and met- 
astatic colorectal cancer patients. Cancer Biother Radiopharm 2005, 20:16-26.

83. Slavin-Chiorini DC, Kashmiri SV, Lee HS, Milenic DE, Poole DJ, Bernon E, Schlom J, Hand PH: A CDR-grafted (humanized) domaindeleted antitumor antibody. Cancer Biother Radiopharm 1997, 12:305-316

84. Fang L, Holford NH, Hinkle G, Cao X, Xiao J], Bloomston M, Gibbs S, Saif OH, Dalton JT, Chan KK, Schlom J, Martin EW Jr, Sun D: Population pharmacokinetics of humanized monoclonal antibody HuCC49deltaCH2 and murine antibody CC49 in colorectal cancer patients. J Clin Pharmacol 2007, 47:227-237.

85. Muraro R, Wunderlich D, Thor A, Lundy J, Noguchi P, Cunningham $R$, Schlom J: Definition by monoclonal antibodies of a repertoire of epitopes on carcinoembryonic antigen differentially expressed in human colon carcinomas versus normal adult tissues. Cancer Res 1985, 45:5769-5780.

86. Hammarström S: The carcinoembryonic antigen (CEA) family: structures, suggested functions and expression in norma and malignant tissues. Semin Cancer Biol 1999, 9:67-81.

87. Goldenberg DM, Kim EE, DeLand FH, Bennett S, Primus FJ: Radioimmunodetection of cancer with radioactive antibodies to carcinoembryonic antigen. Cancer Res 1980, 40:2984-2992.

88. Nap M, Hammarstrom M-L, Borer O, Hammarstrom S, Wagner C Handt S, Schreyer M, Mach JP, Buchegget F, von Kleist S, Grunert F, Seguin P, Fuks A, Holm R, Lamerz R: Specificity and affinity of monoclonal antibodies against carcinoembryonic antigen. Cancer Res 1992, 52:2329-2339.

89. Shi ZR, Tacha D, Itzkowitz SH: Monoclonal antibody COL-I reacts with restricted epitopes on carcinoembryonic antigen: an immunohistochemical study. J Histochem Cytochem 1994, 42:1215-1219.

90. Gu J, Zhao J, Li Z, Yang Z, Zhang J, Gao Z, Wang Y, Xu G: Clinical application of radioimmunoguided surgery in colorectal cancer using |25|-labeled carcinoembryonic antigen-specific monoclonal antibody submucosally. Dis Colon Rectum 2003, 46: $1659-1666$

91. Dawson PM, Blair SD, Begent RH, Kelly AM, Boxer GM, Theodorou $N A$ : The value of radioimmunoguided surgery in first and second look laparotomy for colorectal cancer. Dis Colon Rectum 1991, 34:217-222.

92. Goldenberg DM, Goldenberg H, Sharkey RM, Lee RE, Horowitz JA, Hall TC, Hansen H]: In-vivo antibody imaging for the detection of human tumors. Cancer Treat Res 1990, 51:273-292.

93. Goldenberg DM, Goldenberg H, Sharkey RM, Higginbotham-Ford E, Lee RE, Swayne LC, Burger KA, Tsai D, Horowitz JA, Hall TC, Pinsky $\mathrm{CM}$, Hansen $\mathrm{HJ}$ : Clinical studies of cancer radioimmunodetection with carcinoembryonic antigen monoclonal antibody fragments labeled with I23I or $99 m$ Tc. Cancer Res 1990 50:909s-92Is.

94. Göttlinger HG, Funke I, Johnson JP, Gokel JM, Riethmüller G: The epithelial cell surface antigen I7-IA, a target for antibodymediated tumor therapy: its biochemical nature, tissue distribution and recognition by different monoclonal antibodies. Int J Cancer 1986, 38:47-53

95. Göttlinger H, Johnson J, Riethmüller G: Biochemical and epitope analysis of the I7-IA membrane antigen. Hybridoma 1986, 5(SuppI I):S29-S37.

96. Litvinov SV, Velders MP, Bakker HA, Fleuren GJ, Warnaar SO: EpCAM: a human epithelial antigen is a homophilic cell-cell adhesion molecule. J Cell Biol 1994, I 25:437-446.

97. Leibovitz A, Stinson JC, McCombs WB 3rd, McCoy CE, Mazur KC Mabry ND: Classification of human colorectal adenocarcinoma cell lines. Cancer Res 1976, 36:4562-4569.

98. Packeisen J, Kaup-Franzen C, Knieriem HJ: Detection of surface antigen I7-IA in breast and colorectal cancer. Hybridome 1999, 1 8:37-40.

99. Herlyn M, Steplewski Z, Herlyn D, Koprowski H: Colorectal carcinoma-specific antigen: detection by means of monoclonal antibodies. Proc Natl Acad Sci USA 1979, 76: |438-I442.

100. Herlyn DM, Steplewski Z, Herlyn MF, Koprowski H: Inhibition of growth of colorectal carcinoma in nude mice by monoclonal antibody. Cancer Res 1980, 40:717-72I.

I01. Martin EW Jr, Tuttle SE, Rousseau M, Mojzisik CM, O'Dwyer PJ, Hinkle GH, Miller EA, Goodwin RA, Oredipe OA, Barth RF, Olsen JO Houchens D, Jewell SD, Bucci DM, Adams D, Steplewski Z, Thurston $\mathrm{MO}$ : Radioimmunoguided surgery: intraoperative use of monoclonal antibody I7-IA in colorectal cancer. Hybridoma 1986, 5(SuppI I):S97-SI08.

102. Paganelli G, Magnani P, Fazio F: Pretargeting of carcinomas with the avidin-biotin system. Int J Biol Markers I993, 8: I55-I59.

103. Gruaz-Guyon A, Janevik-Ivanovska E, Raguin O, De Labriolle-Vaylet C, Barbet J: Radiolabeled bivalent haptens for tumor immunodetection and radioimmunotherapy. Q I Nucl Med 200I, 45:201-206

104. Drugs.com ${ }^{\mathrm{TM}}$ : Drug Information Online: Advanced Consumer Information: Radiopharmaceuticals (Diagnostic) [http://www.drugs.com/cons/radiopharmaceuticals-diagnostic.html]

105. Breitz HB, Tyler A, Bjorn MJ, Lesley T, Weiden PL: Clinical experience with Tc-99m nofetumomab merpentan (Verluma) radioimmunoscintigraphy. Clin Nucl Med 1997, 22:615-620.

106. Lechner $P$, Lind $P$, Snyder $M$, Haushofer $H$ : Probe-guided surgery for colorectal cancer. Recent Results Cancer Res 2000, I 57:273-280.

107. Hladik P, Vizda J, Bedrna J, Simkovic D, Strnad L, Smejkal K, Voboril $\mathrm{Z}$ : Immunoscintigraphy and intra-operative radioimmunodetection in the treatment of colorectal carcinoma. Colorectal Dis 2001, 3:380-386.

108. Abdel-Nabi H, Doerr RJ: Clinical applications of indium-I I Ilabeled monoclonal antibody imaging in colorectal cancer patients. Semin Nucl Med 1993, 23:99-II3.

109. Kuhn JA, Nochumson J: Operative probe scintimetry with indium and technetium for colorectal cancer. I Surg Oncol 2007, 96:290-296.

I I0. Warburg O, Posener K, Negelein E: The metabolism of the carcinoma cell. In The Mechanism of Tumors Ist edition. Edited by: Warburg O. New York: Richard R. Smith, Inc; I931:I29-I69.

III. Weber G: Enzymology of cancer cells. N Engl J Med 1977, 296:486-493.

I 12. Merrall NW, Plevin R, Gould GW: Growth factors, mitogens, oncogenes and the regulation of glucose transport. Cell Signal 1993, 5:667-675.

I13. Pauwels EK, Ribeiro MJ, Stoot JH, McCready VR, Bourguignon M, Mazière B: FDG accumulation and tumor biology. Nucl Med Biol 1998, 25:317-322

I 14. Gambhir SS: Molecular imaging of cancer with positron emission tomography. Nat Rev Cancer 2002, 2:683-693.

I I5. Buck AK, Reske SN: Cellular origin and molecular mechanisms of I 8F-FDG uptake: is there a contribution of the endothelium? J Nucl Med 2004, 45:46 I-463.

I 16. Otsuka H, Graham M, Kubo A, Nishitani H: Clinical utility of FDG PET. I Med lnvest 2004, 5 I:I4-19.

I I7. Otsuka H, Morita N, Yamashita K, Nishitani H: FDG-PET/CT for cancer management. J Med Invest 2007, 54:195-199.

I18. The United States Nuclear Regulatory Commission (USNRC) [http://www.nrc.gov/reading-rm/doc-collections/cfr/ part020/part020-|201.html]

I19. ICRP, Publication 60: The 1990 Recommendations of the International Commission on Radiological Protection. Ann ICRP |99|, 2 | (1-3): |-20|.

120. ICRP, Publication 103: The 2007 Recommendations of the International Commission on Radiological Protection (Chapters 5 and 6). Ann ICRP 2007, 37(2-4):8I- 123.

121. Mojzisik C, Loesch J, Hinkle G, Petty L, Berens A, Thurston M, Martin $E$ Jr: An intraoperative assessment of radioactive contamination associated with radioimmunoguided surgery. Association of Operating Room Nurses, Atlanta, GA, April 7-12, 1991

122. Stokkel MP, Boot IN, Smit JW: Personal dosimetry of the staff during treatment of neuroendocrine tumours with a high dose of Indium- I I I Octreotide. Q J Nucl Med 2002, 46:33 I-335.

123. Stratmann SL, McCarty TM, Kuhn JA: Radiation safety with breast sentinel node biopsy. Am J Surg 1999, I 78:454-457.

124. Waddington WA, Keshtgar MR, Taylor I, Lakhani SR, Short MD, Ell $P J:$ Radiation safety of the sentinel lymph node technique in breast cancer. Eur J Nucl Med 2000, 27:377-39l.

125. de Kanter AY, Arends PP, Eggermont AM, Wiggers T: Radiation protection for the sentinel node procedure in breast cancer. Eur J Surg Oncol 2003, 29:396-399.

126. Sera T, Mohos G, Papos M, Osvay M, Varga J, Lazar M, Kiss E, Kapitany K, Dobozy A, Csernay L, Pavics L: Sentinel node detection in malignant melanoma patients: radiation safety considerations. Dermatol Surg 2003, 29:|4|-|45. 
127. Klausen TL, Chakera AH, Friis E, Rank F, Hesse B, Holm S: Radiation doses to staff involved in sentinel node operations for breast cancer. Clin Physiol Funct Imaging 2005, 25:196-202.

I28. Kojima T, Kumita S, Yamaguchi F, Mizumura S, Kitamura T, Kumazaki $\mathrm{T}$, Teramoto A: Radio-guided brain tumorectomy using a gamma detecting probe and a mobile solid-state gamma camera. Surg Neurol 2004, 6 I:229-238. discussion 238

129. Bekiş R, Celik P, Uysal B, Koçdor MA, Atila K, Saydam S, Harmancloğlu $O$, Durak $\mathrm{H}$ : Exposure of surgical staff in surgical probe applications in radioguided parathyroidectomy. Eur Arch Otorhinolaryngol 2008, 265: I545-I548.

130. Heckathorne E, Dimock C, Dahlbom M: Radiation dose to surgical staff from positron-emitter-based localization and radiosurgery of tumors. Health Phys 2008, 95:220-226.

I3I. Andersen PA, Chakera AH, Klausen TL, Binderup T, Grossjohann HS, Friis E, Hansen CP, Schmidt G, Kjaer A, Hesse B: Radiation exposure to surgical staff during F-I 8-FDG-guided cancer surgery. Eur J Nucl Med Mol Imaging 2008, 35:624-629.

132. Povoski SP, Sarikaya I, White WC, Marsh SG, Hall NC, Hinkle GH, Martin EW, Knopp MV: A comprehensive evaluation of occupational radiation exposure to intraoperative and perioperative personnel from 18 F-FDG radioguided surgical procedures. Eur I Nucl Med Mol Imaging 2008, 35:2026-2034.

133. Burak WE, Agnese DM, Povoski SP: Advances in the surgical management of early stage invasive breast cancer. Curr Probl Surg 2004, 41:877-936.

134. National Comprehensive Cancer Network (NCCN) Clinical Guidelines in Oncology for Breast Cancer (V.I.2009) [http:/ /www.nccn.org/professionals/physician gls/PDF/breast.pdf]

135. Krag DN, Weaver DL, Alex JC, Fairbank JT: Surgical resection and radiolocalization of the sentinel lymph node in breast cancer using a gamma probe. Surg Oncol 1993, 2:335-339. discussion 340

136. Wilhelm AJ, Mijnhout GS, Franssen EJF: Radiopharmaceuticals in sentinel lymph-node detection - an overview. Eur J Nucl Med 1999, 26:S36-S42.

137. McCarter MD, Yeung H, Yeh S, Fey J, Borgen PI, Cody HS: Localization of the sentinel node in breast cancer: identical results with same-day and day-before isotope injection. Ann Surg Oncol 2001, 8:682-686.

138. Ent FW van der, Kengen RA, Pol HA van der, Hoofwijk AG: Sentinel node biopsy in 70 unselected patients with breast cancer: increased feasibility by using $10 \mathrm{mCi}$ radiocolloid in combination with a blue dye tracer. Eur J Surg Oncol 1999, 25:24-29.

139. Solorzano CC, Ross MI, Delpassand E, Mirza N, Akins JS, Kuerer HM, Meric F, Ames FC, Newman L, Feig B, Singletary SE, Hunt KK: Utility of breast sentinel lymph node biopsy using day-before-surgery injection of high-dose $99 \mathrm{mTc}$-labeled sulfur colloid. Ann Surg Oncol 200I, 8:821-8I7.

140. Gray RJ, Pockaj BA, Roarke MC: Injection of $(99 \mathrm{~m})$ Tc-labeled sulfur colloid the day before operation for breast cancer sentinel lymph node mapping is as successful as injection the day of operation. Am J Surg 2004, I 88:685-689.

141. Povoski SP, Olsen JO, Young DC, Clarke J, Burak WE, Walker M], Carson WE, Yee LD, Agnese DM, Pozderac RV, Hall NC, Farrar WB: Prospective randomized clinical trial comparing intradermal, intraparenchymal, and subareolar injection routes for sentinel lymph node mapping and biopsy in breast cancer. Ann Surg Oncol 2006, I3:1412-1421.

142. Wong SL, Edwards MJ, Chao C, Tuttle TM, Noyes RD, Carlson DJ, Cerrito PB, McMasters KM: Sentinel lymph node biopsy for breast cancer: impact of the number of sentinel nodes removed on the false-negative rate. J Am Coll Surg 200I, | 92:684-689. discussion 689-69|

143. Martin RC 2nd, Chagpar A, Scoggins CR, Edwards MJ, Hagendoorn L, Stromberg AJ, McMasters KM, University of Louisville Breast Cancer Sentinel Lymph Node Study: Clinicopathologic factors associated with false-negative sentinel lymph-node biopsy in breast cancer. Ann Surg 2005, 24I:1005-1012. discussion 10121015

144. Povoski SP, Young DC, Walker MJ, Carson WE, Yee LD, Agnese DM, Farrar WB: Re-emphasizing the concept of adequacy of intraoperative assessment of the axillary sentinel lymph nodes for identifying nodal positivity during breast cancer surgery. World J Surg Oncol 2007, 5: 18.
145. Woznick A, Franco M, Bendick P, Benitez PR: Sentinel lymph node dissection for breast cancer: how many nodes are enough and which technique is optimal? Am J Surg 2006, I 91:330-333.

146. Wada N, Sakemura N, Imoto S, Hasebe T, Ochiai A, Moriyama N Sentinel node biopsy in primary breast cancer: radioactive detection and metastatic disease. Eur J Surg Oncol 2007, 33:691-695.

147. Krag DN, Anderson SJ, Julian TB, Brown AM, Harlow SP, Ashikaga T, Weaver DL, Miller BJ, Jalovec LM, Frazier TG, Noyes RD, Robidoux A, Scarth HM, Mammolito DM, McCready DR, Mamounas EP, Costantino JP, Wolmark N: National Surgical Adjuvant Breast and Bowel Project: Technical outcomes of sentinel-lymph-node resection and conventional axillary-lymph-node dissection in patients with clinically node-negative breast cancer: results from the NSABP B-32 randomised phase III trial. Lancet Oncol 2007, 8:88I-888.

148. Nieroda CA, Mojzisik C, Sardi A, Farrar WB, Hinkle G, Siddiqi MA, Ferrara PJ, James A, Schlom J, Thurston MO, Martin EW: Staging of carcinoma of the breast using a hand-held gamma detecting probe and monoclonal antibody B72.3. Surg Gynecol Obstet 1989, 169:35-40.

149. Martin EW Jr, Mojzisik CM, Hinkle GH, Sampsel J, Siddiqi M, Tuttle SE, Sickle-Santanello B, Colcher D, Thurston MO, Bell J, Farrar WB, Schlom J: Radioimmunoguided surgery using monoclonal antibody. Am J Surg 1 988, I 56:386-392.

150. Percivale P, Bertoglio S, Meszaros P, Canavese G, Cafiero F, Gipponi $M$, Campora E, Gasco M, Badellino F: Radioimmunoguided surgery after primary treatment of locally advanced breast cancer. I Clin Oncol 1996, I 4: I599-1603.

I5I. Badellino F, Bertoglio S, Mariani G, Meszaros P, Canavese G, Percivale $P$ : Use of radioimmunoguided surgery after induction chemotherapy in locally advanced breast cancer. Semin Surg Oncol 1998, I 5:245-248.

152. Burak WE Jr, DePalatis L, Mosic JL, Soble D, Hinkle G, Hitchcock CL: Radioimmunoguided breast surgery using radiolabeled antibody NR-LU-IO FAB: a pilot study. Tumori 200I, 87:|42-|46.

153. Gennari R, Galimberti V, De Cicco C, Zurrida S, Zerwes F, Pigatto F, Luini A, Paganelli G, Veronesi U: Use of technetium-99m-labeled colloid albumin for preoperative and intraoperative localization of nonpalpable breast lesions. I Am Coll Surg 2000, 190:692-698. discussion 698-699

154. Luini A, Zurrida S, Paganelli G, Galimberti V, Sacchini V, Monti S, Veronesi $\mathrm{P}$, Viale G, Veronesi U: Comparison of radioguided excision with wire localization of occult breast lesions. $\mathrm{Br}$ Surg 1999, 86:522-525.

155. Feggi L, Basaglia E, Corcione S, Querzoli P, Soliani G, Ascanelli S, Prandini N, Bergossi L, Carcoforo P: An original approach in the diagnosis of early breast cancer: use of the same radiopharmaceutical for both non-palpable lesions and sentinel node localisation. Eur J Nucl Med 200I, 28:1589-I596.

I56. Gray RJ, Giuliano R, Dauway EL, Cox CE, Reintgen DS: Radioguidance for nonpalpable primary lesions and sentinel lymph node(s). Am J Surg 200 I, I 82:404-406.

157. Tanis PJ, Deurloo EE, Valdés Olmos RA, Rutgers EJ, Nieweg OE, Besnard AP, Kroon BB: Single intralesional tracer dose for radio-guided excision of clinically occult breast cancer and sentinel node. Ann Surg Oncol 200I, 8:850-855.

158. De Cicco C, Pizzamiglio M, Trifiro G, Luini A, Ferrari M, Prisco G, Galimberti V, Cassano E, Viale G, Intra M, Veronesi P, Paganelli G: Radioguided occult lesion localisation (ROLL) and surgical biopsy in breast cancer. Technical aspects. Q J Nucl Med 2002 , 46: $|45-| 5 \mid$.

I59. Gray RJ, Pockaj BA, Karstaedt PJ, Roarke MC: Radioactive seed localization of nonpalpable breast lesions is better than wire localization. Am J Surg 2004, I 88:377-380.

160. Rampaul RS, Bagnall M, Burrell H, Pinder SE, Evans AJ, Macmillan RD: Randomized clinical trial comparing radioisotope occult lesion localization and wire-guided excision for biopsy of occult breast lesions. Br J Surg 2004, 9 I:1575-1577.

16I. Zgajnar J, Hocevar M, Frkovic-Grazio S, Hertl K, Schweiger E, Besic N: Radioguided occult lesion localization (ROLL) of the nonpalpable breast lesions. Neoplasma 2004, 5 I:385-389.

162. Patel A, Pain S], Britton P, Sinnatamby R, Warren R, Bobrow L, Barber RW, Peters AM, Purushotham AD: Radioguided occult lesion localisation (ROLL) and sentinel node biopsy for impalpable invasive breast cancer. Eur J Surg Oncol 2004, 30:918-923. 
163. Ronka R, Krogerus L, Leppanen E, von Smitten K, Leidenius M: Radio-guided occult lesion localization in patients undergoing breast-conserving surgery and sentinel node biopsy. Am J Surg 2004, 187:491-496.

164. Audisio RA, Nadeem R, Harris O, Desmond S, Thind R, Chagla LS: Radioguided occult lesion localisation (ROLL) is available in the UK for impalpable breast lesions. Ann R Coll Surg Engl 2005, 87:92-95.

165. Thind CR, Desmond S, Harris O, Nadeem R, Chagla LS, Audisio RA: Radio-guided localization of clinically occult breast lesions (ROLL): a DGH experience. Clin Radiol 2005, 60:68I-686.

166. Nadeem R, Chagla LS, Harris O, Desmond S, Thind R, Titterrell C, Audisio RA: Occult breast lesions: A comparison between radioguided occult lesion localisation (ROLL) vs. wire-guided lumpectomy (WGL). Breast 2005, | 4:283-289.

167. Duarte GM, dos Santos CC, Torresan RZ, Alvarenga M, Telles GH, Bianchessi ST, Caserta N, Segala SR, Lopes de Lima Mda C, de Camargo Etchebehere EC, Camargo EE: Radioguided surgery using intravenous $99 \mathrm{mTc}$ sestamibi associated with breast magnetic resonance imaging for guidance of breast cancer resection. Breast J 2006, I 2:202-207.

168. Monti S, Galimberti V, Trifiro G, De Cicco C, Peradze N, Brenelli F, Fernandez-Rodriguez J, Rotmensz N, Latronico A, Berrettini A, Mauri M, Machado L, Luini A, Paganelli G: Occult breast lesion localization plus sentinel node biopsy (SNOLL): experience with 959 patients at the European Institute of Oncology. Ann Surg Oncol 2007, I 4:2928-293I.

169. Medina-Franco H, Abarca-Perez L, Ulloa-Gomez JL, Romero C: Radioguided localization of clinically occult breast lesions (ROLL): a pilot study. Breast J 2007, I 3:40 I-405.

170. van Rijk MC, Tanis PJ, Nieweg OE, Loo CE, Olmos RA, Oldenburg HS, Rutgers EJ, Hoefnagel CA, Kroon BB: Sentinel node biopsy and concomitant probe-guided tumor excision of nonpalpable breast cancer. Ann Surg Oncol 2007, 14:627-632.

I7I. Barros AC, Barros MA, Andrade FE, Mori LJ, Costa PA, Sheng PY, Pelizon $\mathrm{CH}$ : Combined radioguided nonpalpable lesion localization and sentinel lymph node biopsy for early breast carcinoma. Ann Surg Oncol 2007, I 4: |472-I477.

172. Panareo S, Carcoforo P, Lanzara S, Corcione S, Bagatin E, Casali M, Costanzo A, Basaglia E, Feggi LM: Radiolabelled somatostatin analogs for diagnosis and radio-guided surgery of neuroendocrine breast cancer undetectable with conventional imaging procedures. Breast 2007, I 7: I I I-I I4.

173. Machado RH, Oliveira AC, Rocha AC, Landesmann MC, Martins FP, Lopes SA, Gutfilen B, da Fonseca LM: Radioguided occult lesion localization (ROLL) and excision of breast lesions using technetium-99m-macroaggregate albumin and air injection control. I Exp Clin Cancer Res 2007, 26:323-327.

174. Paredes P, Vidal-Sicart S, Zanón G, Roé N, Rubí S, Lafuente S, Pavía J, Pons F: Radioguided occult lesion localisation in breast cancer using an intraoperative portable gamma camera: first results. Eur J Nucl Med Mol lmaging 2007, 35:230-235.

175. Medina-Franco H, Abarca-Pérez L, García-Alvarez MN, Ulloa-Gómez JL, Romero-Trejo C, Sepúlveda-Méndez J: Radioguided occult lesion localization (ROLL) versus wire-guided lumpectomy for non-palpable breast lesions: a randomized prospective evaluation. J Surg Oncol 2008, 97:I08-III.

176. Ploeg IM van der, Hobbelink M, Bosch MA van den, Mali WP, Rinkes IH, van Hillegersberg R: Radioguided occult lesion localisation' (ROLL) for non-palpable breast lesions: A review of the relevant literature. Eur J Surg Oncol 2008, 34: I-5.

177. Lavoué $V$, Nos $C$, Clough KB, Baghaie F, Zerbib E, Poulet B, Lefrère Belda MA, Ducellier A, Lecuru F: Simplified technique of radioguided occult lesion localization (ROLL) plus sentinel lymph node biopsy (SNOLL) in breast carcinoma. Ann Surg Oncol 2008, I 5:2556-256I.

178. Van Esser S, Hobbelink M, Ploeg IM Van der, Mali WP, Van Diest PJ, Borel Rinkes IH, Van Hillegersberg R: Radio guided occult lesion localization (ROLL) for non-palpable invasive breast cancer. J Surg Oncol 2008, 98:526-529.

179. Moreno M, Wiltgen JE, Bodanese B, Schmitt RL, Gutfilen B, da Fonseca LM: Radioguided breast surgery for occult lesion localization - correlation between two methods. J Exp Clin Cancer Res 2008, 27:29

180. Sarlos D, Frey LD, Haueisen H, Landmann G, Kots LA, Schaer G: Radioguided occult lesion localization (ROLL) for treatment and diagnosis of malignant and premalignant breast lesions combined with sentinel node biopsy: A prospective clinical trial with 100 patients. Eur J Surg Oncol 2008 in press.

18I. Ramesh HS, Anguille S, Chagla LS, Harris O, Desmond S, Thind R, Audisio RA: Recurrence after ROLL lumpectomy for invasive breast cancer. Breast 2008, 17:637-639.

182. van Esser S, Hobbelink MG, Peeters PH, Buskens E, Ploeg IM van der, Mali WP, Borel Rinkes IH, van Hillegersberg R: The efficacy of 'Radio guided Occult Lesion Localization' (ROLL) versus 'Wire-guided Localization' (WGL) in breast conserving surgery for non-palpable breast cancer: a randomized clinical trial - ROLL study. BMC Surg 2008, 8:9.

183. Cuntz MC, Levine EA, O'Dorisio TM, Watson JC, Wray DA, Espenan GD, McKnight C, Meier JR, Weber LJ, Mera R, O'Dorisio MS, Woltering EA: Intraoperative gamma detection of I 25I-lanreotide in women with primary breast cancer. Ann Surg Oncol 1999, 6:367-372.

184. Duarte GM, Cabello C, Torresan RZ, Alvarenga M, Telles GH, Bianchessi ST, Caserta N, Segala SR, de Lima MC, Etchebehere EC, Camargo EE: Radioguided Intraoperative Margins Evaluation (RIME): Preliminary results of a new technique to aid breast cancer resection. Eur J Surg Oncol 2007, 33: I I 50- I I 57.

185. National Comprehensive Cancer Network (NCCN) Clinical Guidelines in Oncology for melanoma (V.2.2009) [http:// www.nccn.org/professionals/physician gls/PDF/melanoma.pdf]

186. Morton DL, Wen DR, Wong JH, Economou JS, Cagle LA, Storm FK Foshag LJ, Cochran AJ: Technical details of intraoperative lymphatic mapping for early stage melanoma. Arch Surg 1992 , I 27:392-399.

187. Uren RF, Howman-Giles RB, Shaw HM, Thompson JF, McCarthy WH: Lymphoscintigraphy in high-risk melanoma of the trunk: predicting draining node groups, defining lymphatic channels and locating the sentinel node. I Nucl Med 1993, 34: $1435-1440$.

188. Alex JC, Weaver DL, Fairbank JT, Rankin BS, Krag DN: Gammaprobe-guided lymph node localization in malignant melanoma. Surg Oncol 1993, 2:303-308.

189. Albertini JJ, Cruse CW, Rapaport D, Wells K, Ross M, DeConti R, Berman CG, Jared K, Messina J, Lyman G, Glass F, Fenske N, Reintgen DS: Intraoperative radio-lympho-scintigraphy improves sentinel lymph node identification for patients with melanoma. Ann Surg 1996, 223:217-224.

190. Glass LF, Messina JL, Cruse W, Wells K, Rapaport D, Miliotes G, Berman $C$, Reintgen D, Fenske NA: The use of intraoperative radiolymphoscintigraphy for sentinel node biopsy in patients with malignant melanoma. Dermatol Surg 1996, 22:715-720.

191. Pijpers R, Borgstein PJ, Meijer S, Hoekstra OS, van Hattum LH, Teule GJ: Sentinel node biopsy in melanoma patients: dynamic lymphoscintigraphy followed by intraoperative gamma probe and vital dye guidance. World J Surg 1997, 2 I:788-792. discussion 793

192. Gershenwald JE, Tseng CH, Thompson W, Mansfield PF, Lee JE, Bouvet $M$, Lee J], Ross MI: Improved sentinel lymph node localization in patients with primary melanoma with the use of radiolabeled colloid. Surgery 1998, I 24:203-210.

193. Morton DL, Thompson JF, Essner R, Elashoff R, Stern SL, Nieweg OE, Roses DF, Karakousis CP, Mozzillo N, Reintgen D, Wang HJ, Glass EC, Cochran AJ: Validation of the accuracy of intraoperative lymphatic mapping and sentinel lymphadenectomy for early-stage melanoma: a multicenter trial. Multicenter Selective Lymphadenectomy Trial Group. Ann Surg 1999, 230:453-463. discussion 463-465

194. Landi G, Polverelli M, Moscatelli G, Morelli R, Landi C, Fiscelli O, Erbazzi A: Sentinel lymph node biopsy in patients with primary cutaneous melanoma: study of $\mathbf{4 5 5}$ cases. J Eur Acad Dermatol Venereol 2000, I 4:35-45.

195. Pijpers R, Borgstein PJ, Teule G], Meijer S: Vital dye and radiolabelled colloids - complement or alternative? Recent Results Cancer Res 2000, 157:130-137.

196. Povoski SP, Choudry UH, Dauway EL, Rassekh CH, Ducatman BS: Sentinel lymph node mapping and biopsy for malignant melanoma at a rural-based university medical center. $W V$ Med ] 2002, 98: I 94- 197.

197. Ariyan S, Ariyan C, Farber LR, Fischer DS, Flynn SD, Truini C: Reliability of identification of 655 sentinel lymph nodes in 263 con- 
secutive patients with malignant melanoma. I Am Coll Surg 2004, 198:924-932.

198. Chakera AH, Drzewiecki KT, Eigtved A, Juhl BR: Sentinel node biopsy for melanoma: a study of 24I patients. Melanoma Res 2004, I 4:52I-526.

199. Krag D, Harlow S, Weaver D, Ashikaga T: Technique of sentinel node resection in melanoma and breast cancer: probeguided surgery and lymphatic mapping. Eur J Surg Oncol 1998, 24:89-93.

200. Kelley MC, Ollila DW, Morton DL: Lymphatic mapping and sentinel lymphadenectomy for melanoma. Semin Surg Oncol 1998 14:283-290.

20I. Cochran AJ, Balda BR, Starz H, Bachter D, Krag DN, Cruse CW, Pijpers R, Morton DL: The Augsburg Consensus. Techniques of lymphatic mapping, sentinel lymphadenectomy, and completion lymphadenectomy in cutaneous malignancies. Cancer 2000, 89:236-24I.

202. Clary BM, Brady MS, Lewis J], Coit DG: Sentinel Iymph node biopsy in the management of patients with primary cutaneous melanoma: review of a large single-institutional experience with an emphasis on recurrence. Ann Surg 2001, 233:250-258.

203. Intenzo CM, Kim SM, Patel JI, Lin HC, Kairys JC: Lymphoscintigraphy in cutaneous melanoma: a total body atlas of sentinel node mapping. Radiographics 2002, 22:491-502.

204. Perrott RE, Glass LF, Reintgen DS, Fenske NA: Reassessing the role of lymphatic mapping and sentinel lymphadenectomy in the management of cutaneous malignant melanoma. I Am Acad Dermatol 2003, 49:567-588.

205. Landry CS, McMasters KM, Scoggins CR: The evolution of the management of regional lymph nodes in melanoma. I Surg Oncol 2007, 96:316-321.

206. Amersi F, Morton DL: The role of sentinel lymph node biopsy in the management of melanoma. Adv Surg 2007, 4I:24I-256.

207. Porter GA, Ross MI, Berman RS, Lee JE, Mansfield PF, Gershenwald JE: Significance of multiple nodal basin drainage in truncal melanoma patients undergoing sentinel lymph node biopsy. Ann Surg Oncol 2000, 7:256-26I.

208. Jansen L, Nieweg OE, Peterse JL, Hoefnagel CA, Olmos RA, Kroon $B B$ : Reliability of sentinel lymph node biopsy for staging melanoma. Br J Surg 2000, 87:484-489.

209. Jacobs IA, Chang CK, Salti GI: Significance of dual-basin drainage in patients with truncal melanoma undergoing sentinel lymph node biopsy. J Am Acad Dermatol 2003, 49:615-619.

210. Leong SP, Morita ET, Südmeyer M, Chang J, Shen D, Achtem TA Allen RE Jr, Kashani-Sabet M: Heterogeneous patterns of lymphatic drainage to sentinel lymph nodes by primary melanoma from different anatomic sites. Clin Nucl Med 2005, 30:150-158

211. Jimenez RE, Panageas K, Busam KJ, Brady MS: Prognostic implications of multiple lymphatic basin drainage in patients with truncal melanoma. J Clin Oncol 2005, 23:5 I8-524.

212. McHugh JB, Su L, Griffith KA, Schwartz JL, Wong SL, Cimmino V Chang AE, Johnson TM, Sabel MS: Significance of multiple lymphatic basin drainage in truncal melanoma patients undergoing sentinel lymph node biopsy. Ann Surg Oncol 2006 , 13:1216-1223.

213. Vucetić B, Andreja Rogan S, Balenović A, Ivkić M, Situm M, Hudorović $\mathrm{N}$, Kusić Z: The role of preoperative lymphoscintigraphy in surgery planning for sentinel lymph node biopsy in malignant melanoma. Wien Klin Wochenschr 2006, I I 8:286-293.

214. Federico AC, Chagpar AB, Ross MI, Martin RC, Noyes RD, Goydos JS, Beitsch PD, Urist MM, Ariyan S, Sussman JJ, McMasters KM, Scoggins CR, Sunbelt Melanoma Trial: Effect of multiple-nodal basin drainage on cutaneous melanoma. Arch Surg 2008 | 43:632-637. discussion 637-638

215. Wong JH, Truelove K, Ko P, Coel MN: Localization and resection of an in transit sentinel lymph node by use of lymphoscintigraphy, intraoperative lymphatic mapping, and a hand-held gamma probe. Surgery 1996, I20:1|4-||6.

216. Uren RF, Howman-Giles R, Thompson JF, McCarthy WH, Quinn MJ, Roberts JM. Shaw HM: Interval nodes: the forgotten sentine nodes in patients with melanoma. Arch Surg 2000 I35: I 168-II72.

2 17. Thelmo MC, Morita ET, Treseler PA, Nguyen LH, Allen RE Jr, Sagebie RW, Kashani-Sabet M, Leong SP: Micrometastasis to in-transit lymph nodes from extremity and truncal malignant melanoma. Ann Surg Oncol 200I, 8:444-448.

218. McMasters KM, Chao C, Wong SL, Wrightson WR, Ross MI, Reintgen DS, Noyes RD, Cerrito PB, Edwards MJ, Sunbelt Melanoma Trial Group: Interval sentinel lymph nodes in melanoma. Arch Surg 2002, I 37:543-547.

219. Vidal-Sicart S, Pons F, Fuertes S, Vilalta A, Rull R, Puig S, Palou JM, Ortega $M$, Castel $T$ : Is the identification of in-transit sentinel lymph nodes in malignant melanoma patients really necessary? Eur J Nucl Med Mol Imaging 2004, 3 I:945-949.

220. Nathanson SD: Will the true sentinel node please stand? Ann Surg Oncol 1999, 6:514-516.

22I. McMasters KM, Reintgen DS, Ross MI, Wong SL, Gershenwald JE, Krag DN, Noyes RD, Viar V, Cerrito PB, Edwards MJ: Sentinel lymph node biopsy for melanoma: how many radioactive nodes should be removed? Ann Surg Oncol 2001, 8: 192-197.

222. Carlson GW, Murray DR, Thourani V, Hestley A, Cohen C: The definition of the sentinel lymph node in melanoma based on radioactive counts. Ann Surg Oncol 2002, 9:929-933.

223. Jacobs IA, Chang CK, DasGupta TK, Salti G: High isotope counts and sentinel node positivity in patients with melanoma. Arch Surg 2003, 138:63-66. discussion 67

224. Kroon HM, Lowe L, Wong S, Fullen D, Su L, Cimmino V, Chang AE Johnson T, Sabel MS: What is a sentinel node? Re-evaluating the $10 \%$ rule for sentinel lymph node biopsy in melanoma. J Surg Oncol 2007, 95:623-628.

225. Emery RE, Stevens JS, Nance RW, Corless CL, Vetto JT: Sentinel node staging of primary melanoma by the "10\% rule": pathology and clinical outcomes. Am J Surg 2007, 193:6 |8-622. discussion 622

226. Esmaeli B: Sentinel lymph node mapping for patients with cutaneous and conjunctival malignant melanoma. Ophthal Plast Reconstr Surg 2000, I 6: I70-I72.

227. Wilson MW, Fleming JC, Fleming RM, Haik BG: Sentinel node biopsy for orbital and ocular adnexal tumors. Ophthal Plast Reconstr Surg 2001, I 7:338-344. discussion 344-345

228. Esmaeli B, Eicher S, Popp J, Delpassand E, Prieto VG, Gershenwald JE: Sentinel lymph node biopsy for conjunctival melanoma. Ophthal Plast Reconstr Surg 200I, I 7:436-442.

229. Amato M, Esmaeli B, Ahmadi MA, Tehrani MH, Gershenwald J, Ross $M$, Holds J, Delpassand E: Feasibility of preoperative lymphoscintigraphy for identification of sentinel lymph nodes in patients with conjunctival and periocular skin malignancies. Ophthal Plast Reconstr Surg 2003, 19:102-106.

230. Esmaeli B, Reifler D, Prieto VG, Amir Ahmadi M, Hidaji L, Delpassand $E$, Ross MI: Conjunctival melanoma with a positive sentinel Iymph node. Arch Ophthalmol 2003, I 2 I: I 779- I783.

23I. Nijhawan N, Ross MI, Diba R, Ahmadi MA, Esmaeli B: Experience with sentinel lymph node biopsy for eyelid and conjunctival malignancies at a cancer center. Ophthal Plast Reconstr Surg 2004, 20:291-295

232. Baroody M, Holds JB, Kokoska MS, Boyd J: Conjunctival melanoma metastasis diagnosed by sentinel lymph node biopsy. Am J Ophthalmol 2004, I37: I | 47-I I 49

233. Ho VH, Ross MI, Prieto VG, Khaleeq A, Kim S, Esmaeli B: Sentinel lymph node biopsy for sebaceous cell carcinoma and melanoma of the ocular adnexa. Arch Otolaryngol Head Neck Surg 2007, I 33:820-82.

234. Schwarz KA, Davison SP, Crane AE: Sentinel lymph node biopsy in the setting of conjunctival melanoma. Plast Reconstr Surg 2008, I 2 I:2I $2 \mathrm{e}-2 \mid 3 \mathrm{e}$

235. de Hullu JA, Hollema H, Hoekstra HJ, Piers DA, Mourits MJ, Aalders JG, Zee AG van der: Vulvar melanoma: is there a role for sentinel lymph node biopsy? Cancer 2002, 94:486-491.

236. Abramova L, Parekh J, Irvin WP Jr, Rice LW, Taylor PT Jr, Anderson WA, Slingluff CL Jr: Sentinel node biopsy in vulvar and vaginal melanoma: presentation of six cases and a literature review. Ann Surg Oncol 2002, 9:840-846.

237. Wechter ME, Gruber SB, Haefner HK, Lowe L, Schwartz JL, Reynolds $\mathrm{KR}$, Johnston CM, Johnson TM: Vulvar melanoma: a report of 20 cases and review of the literature. J Am Acad Dermatol 2004, 50:554-562.

238. Dhar KK, DAS N, Brinkman DA, Beynon JL, Woolas RP: Utility of sentinel node biopsy in vulvar and vaginal melanoma: report of two cases and review of the literature. Int I Gynecol Cancer 2007, 17:720-723. 
239. Migliano E, Monarca C, Mariani L, Tedesco M, Bucher S: Vulvar primitive melanoma and sentinel lymph node: case report. Eur J Gynaecol Oncol 2007, 28:229-232.

240. Ayhan A, Celik H, Dursun P: Lymphatic mapping and sentinel node biopsy in gynecological cancers: a critical review of the literature. World J Surg Oncol 2008, 6:53.

24I. Rodier JF, Janser JC, David E, Routiot T, Ott G: Radiopharmaceutical-guided surgery in primary malignant melanoma of the vagina. Gynecol Oncol 1999, 75:308-309.

242. Descheemaeker V, Garin E, Morcel K, Lesimple T, Burtin F, Levêque $\mathrm{J}$ : Radioisotopic location of the sentinel node in vaginal mucous melanoma before laparoscopic sampling. Surg Laparosc Endosc Percutan Tech 2008, I 8:195-196.

243. Tien HY, McMasters KM, Edwards MJ, Chao C: Sentinel lymph node metastasis in anal melanoma: a case report. Int J Gastrointest Cancer 2002, 2:53-56.

244. Bobin JY, Gérard JP, Chapet O, Romestaing P, Isaac S: [Lymphatic mapping and inguinal sentinel lymph node biopsy in anal canal cancers to avoid prophylactic inguinal irradiation]. Cancer Radiother 2003, 7(Suppl I):85s-90s. [French]

245. Olsha O, Mintz A, Gimon Z, Gold Deutch R, Rabin I, Halevy A, Reissman P: Anal melanoma in the era of sentinel lymph node mapping: a diagnostic and therapeutic challenge. Tech Coloproctol 2005, 9:60-62.

246. Sanli Y, Turkmen C, Kurul S, Taş F, Mudun A, Cantez S: Sentinel lymph node biopsy for the staging of anal melanoma: report of two cases. Ann Nucl Med 2006, 20:629-63I.

247. Duport C, Tiffet O, Perrot JL, Prévot N, Rey Y, Cambazard F: [Sentinel node mapping in anorectal melanoma]. Ann Chir 2006 , | 3 |:550-552. [French]

248. Mariolis-Sapsakos T, Malamitsi J, Yakoumakis E, Orfanos F: Is sentinel node mapping useful in anorectal melanoma? Hell J Nuc Med 2008, I I:39-42

249. Argenyi EE, Dogan AS, Urdaneta LF, Ponto LL, Hichwa RD, Watkins GL: Detection of unsuspected metastasis in a melanoma patient with positron emission tomography. Clin Nud Med 1995, 20:744-747.

250. Steinert HC, Huch Böni RA, Buck A, Böni R, Berthold T, Marincek B, Burg G, von Schulthess GK: Malignant melanoma: staging with whole-body positron emission tomography and 2-[F-I8] fluoro-2-deoxy-D-glucose. Radiology 1995, I95:705-709.

25I. Damian DL, Fulham MJ, Thompson E, Thompson JF: Positron emission tomography in the detection and management of metastatic melanoma. Melanoma Res 1996, 6:325-329.

252. Brady MS, Akhurst T, Spanknebel K, Hilton S, Gonen M, Patel A, Larson S: Utility of preoperative [( I 8)]f fluorodeoxyglucose-positron emission tomography scanning in high-risk melanoma patients. Ann Surg Oncol 2006, I 3:525-532.

253. Reinhardt MJ, Joe AY, Jaeger U, Huber A, Matthies A, Bucerius J, Roedel R, Strunk H, Bieber T, Biersack HJ, Tüting T: Diagnostic performance of whole body dual modality I8F-FDG PET/CT imaging for $\mathbf{N}$ - and $\mathrm{M}$-staging of malignant melanoma: experience with 250 consecutive patients. I Clin Oncol 2006, 24: $1178-1187$

254. Hsueh EC, Essner R, Foshag LJ, Ollila DW, Gammon G, O'Day SJ, Boasberg PD, Stern SL, Ye X, Morton DL: Prolonged survival after complete resection of disseminated melanoma and active immunotherapy with a therapeutic cancer vaccine. I Clin Oncol 2002, 20:4549-4554.

255. National Comprehensive Cancer Network (NCCN) Clinical Guidelines in Oncology for Merkel Cell Carcinoma (V.I.2009) [http://www.nccn.org/professionals/physician gls/PDF/ mcc.pdf]

256. Bichakjian CK, Lowe L, Lao CD, Sandler HM, Bradford CR, Johnson TM, Wong SL: Merkel cell carcinoma: critical review with guidelines for multidisciplinary management. Cancer 2007 i 10:1-12.

257. Messina JL, Reintgen DS, Cruse CW, Rappaport DP, Berman C, Fenske NA, Glass LF: Selective lymphadenectomy in patients with Merkel cell (cutaneous neuroendocrine) carcinoma. Ann Surg Oncol 1997, 4:389-395.

258. Ames SE, Krag DN, Brady MS: Radiolocalization of the sentinel lymph node in Merkel cell carcinoma: a clinical analysis of seven cases. J Surg Oncol 1998, 67:251-254.

259. Bilchik AJ, Giuliano A, Essner R, Bostick P, Kelemen P, Foshag LJ, Sostrin S, Turner RR, Morton DL: Universal application of intraop- erative lymphatic mapping and sentinel lymphadenectomy in solid neoplasms. Cancer J Sci Am 1998, 4:351-358.

260. Hill AD, Brady MS, Coit DG: Intraoperative lymphatic mapping and sentinel lymph node biopsy for Merkel cell carcinoma. $\mathrm{Br}$ J Surg 1999, 86:518-521.

26I. Sian KU, Wagner JD, Sood R, Park HM, Havlik R, Coleman J]: Lymphoscintigraphy with sentinel lymph node biopsy in cutaneous Merkel cell carcinoma. Ann Plast Surg 1999, 42:679-682.

262. Zeitouni NC, Cheney RT, Delacure MD: Lymphoscintigraphy, sentinel lymph node biopsy, and Mohs micrographic surgery in the treatment of Merkel cell carcinoma. Dermatol Surg 2000, 26:12-18.

263. Kurul S, Mudun A, Aksakal N, Aygen M: Lymphatic mapping for Merkel cell carcinoma. Plast Reconstr Surg 2000, I 05:680-683.

264. Allen PJ, Busam K, Hill AD, Stojadinovic A, Coit DG: Immunohistochemical analysis of sentinel lymph nodes from patients with Merkel cell carcinoma. Cancer 200I, 92: I650-I655.

265. Rodrigues LK, Leong SP, Kashani-Sabet M, Wong JH: Early experience with sentinel lymph node mapping for Merkel cell carcinoma. I Am Acad Dermatol 200I, 45:303-308.

266. Düker I, Starz H, Bachter D, Balda BR: Prognostic and therapeutic implications of sentinel lymphonodectomy and S-staging in Merkel cell carcinoma. Dermatology 200I, 202:225-229.

267. Blom A, Kolb F, Lumbroso J, Duvillard P, Mamelle G, Morzli K, Ricard M, Spatz A, Petrow P, Margulis A, Avril MF: [Significance of sentinel lymph node biopsy in Merkel cell carcinoma. Analysis of I I cases]. Ann Dermatol Venereol 2003, I30:4 17-422. [French]

268. Wagner JD, Evdokimow DZ, Weisberger E, Moore D, Chuang TY Wenck S, Coleman IJ 3rd: Sentinel node biopsy for high-risk nonmelanoma cutaneous malignancy. Arch Dermatol 2004, I 40:75-79.

269. Michl C, Starz H, Bachter D, Balda BR: Sentinel lymphonodectomy in nonmelanoma skin malignancies. Br J Dermatol 2003 I 49:763-769.

270. Alex JC: The application of sentinel node radiolocalization to solid tumors of the head and neck: a 10-year experience. Laryngoscope 2004, I | 4:2-19.

27I. Schmalbach CE, Lowe L, Teknos TN, Johnson TM, Bradford CR: Reliability of sentinel lymph node biopsy for regional staging of head and neck Merkel cell carcinoma. Arch Otolaryngol Head Neck Surg 2005, I 3 I:6 10-6 I4.

272. Maza S, Trefzer U, Hofmann M, Schneider S, Voit C, Krössin T, Zander A, Audring H, Sterry W, Munz DL: Impact of sentinel lymph node biopsy in patients with Merkel cell carcinoma: results of a prospective study and review of the literature. Eur J Nucl Med Mol Imaging 2006, 33:433-440.

273. Ortin-Perez J, van Rijk MC, Valdes-Olmos RA, Vidal-Sicart S, Nieweg $\mathrm{OE}$, Vilalta $\mathrm{A}$, Kroon BB, Pons F: Lymphatic mapping and sentinel node biopsy in Merkel's cell carcinoma. Eur J Surg Oncol 2007, 33: I19-122.

274. Migliano E, Monarca C, Tedesco M, Rizzo MI, Bucher S: [Merkel cell carcinoma and sentinel lymph node dissection: nine cases report]. G Chir 2008, 29:28-32. [Italian]

275. Argenzio V, Rambaldi PF, lorio G, Cuccurullo V, Cascini GL, Argenziano G, Mansi L: Radioimmunoguided surgery in squamous cell carcinoma. Plast Reconstr Surg 1999, 103:749-75I.

276. Nouri K, Rivas MP, Pedroso F, Bhatia R, Civantos F: Sentinel lymph node biopsy for high-risk cutaneous squamous cell carcinoma of the head and neck. Arch Dermatol 2004, | 40: | 284.

277. Civantos FJ, Moffat FL, Goodwin WJ: Lymphatic mapping and sentinel lymphadenectomy for 106 head and neck lesions: contrasts between oral cavity and cutaneous malignancy. Laryngoscope 2006, I I 2(3 Pt 2 Suppl I09): I- I5.

278. Frerich B, Förster M, Schiefke F, Wittekind C, Hemprich A, Sabri O Sentinel lymph node biopsy in squamous cell carcinomas of the lips and the oral cavity - a single center experience. J Surg Oncol 2007, 95:97-105.

279. Stadelmann WK, Javaheri S, Cruse CW, Reintgen DS: The use of selective lymphadenectomy in squamous cell carcinoma of the wrist: a case report. I Hand Surg [Am] 1997, 22:726-73 I.

280. Reschly MJ, Messina JL, Zaulyanov LL, Cruse W, Fenske NA: Utility of sentinel lymphadenectomy in the management of patients with high-risk cutaneous squamous cell carcinoma. Dermatol Surg 2003, 29: | 35- | 40 
28I. Ozçelik D, Tatlidede S, Hacikerim S, Uğurlu K, Atay M: The use of sentinel lymph node biopsy in squamous cell carcinoma of the foot: a case report. J Foot Ankle Surg 2004, 43:60-63.

282. Perez-Naranjo L, Herrera-Saval A, Garcia-Bravo B, Perez-Bernal AM, Camacho F: Sentinel lymph node biopsy in recessive dystrophic epidermolysis bullosa and squamous cell carcinoma. Arch Dermatol 2005, I 4I: I I0-III.

283. Cuccia G, Colonna MR, Papalia I, Manasseri B, Romeo M, d'Alcontres FS: The use of sentinel node biopsy and selective lymphadenectomy in squamous cell carcinoma of the upper limb. Usefulness of sentinel node biopsy to avoid useless lymphadenectomies in high-risk upper limb SCC. Ann Ital Chir 2008, 79:67-7I.

284. Harwood M, Wu H, Tanabe K, Bercovitch L: Metastatic basal cell carcinoma diagnosed by sentinel lymph node biopsy. J Am Acad Dermatol 2005, 53:475-478.

285. Cózar MP, Ferrer-Rebolleda J, Redal MC, Moreno A, Tortajada L, Casáns I, Romero C: [Sentinel lymph node biopsy in cutaneous non-melanoma malignancies]. Rev Esp Med Nucl 2006, 25: I0-I4. [Spanish]

286. Delgado R, Kraus D, Coit DG, Busam KJ: Sentinel lymph node analysis in patients with sweat gland carcinoma. Cancer 2003 97:2279-2284

287. Bogner PN, Fullen DR, Lowe L, Paulino A, Biermann JS, Sondak VK Su LD: Lymphatic mapping and sentinel lymph node biopsy in the detection of early metastasis from sweat gland carcinoma. Cancer 2003, 97:2285-2289.

288. Wasserman DI, Sack J, Gonzalez-Serva A, Konnikov N, Bohac G Demierre MF: Sentinel lymph node biopsy for a squamoid eccrine carcinoma with lymphatic invasion. Dermatol Surg 2007, 33: I I26-1 I29.

289. Aitken DR, Thurston MO, Hinkle GH Jr, Martin DT, Haagensen DE Jr, Houchens D, Tuttle SE, Martin EW Jr: Portable gamma probe for radioimmune localization of experimental colon tumor xenografts. J Surg Res 1984, 36:480-489.

290. Aitken DR, Hinkle GH, Thurston MO, Tuttle SE, Martin DT, Olsen J, Haagensen DE Jr, Houchens D, Martin EW Jr: A gamma-detecting probe for radioimmune detection of CEA-producing tumors. Successful experimental use and clinical case report. Dis Colon Rectum 1984, 27:279-282.

29I. Martin DT, Hinkle GH, Tuttle S, Olsen J, Nabi H, Houchens D, Thurston M, Martin EW Jr: Intraoperative radioimmunodetection of colorectal tumors with a hand-held radiation detec tor. Am J Surg 1985, 150:672-675.

292. Martin EW Jr, Hinkle G, Mojzisik C, Thurston MO: Radioimmunoguided surgery: a new intraoperative approach to the detection of tumor. Cancer Treat Res 1990, 5 I:387-4I I.

293. Hinkle G, Houchens D, Miller E, Nines R, Nabi H, Thurston M, Tuttle S, Mojzisik C, Martin EW Jr: Preferential localization of antibody combinations in tumor xenografts in nude mice. In ImmuneDeficient Animals in Biomedical Research 5th edition. Edited by: Rygaard, Brûnner, Graem, Spang-Thomsen. Workshop on Immune-Deficient Animals, Cophenhagen; 1987:248-250.

294. O'Dwyer PJ, Mojzisik CM, Hinkle GH, Tuttle SE, Rousseau M, Olsen J, Tuttle SE, Barth RF, Houchens, Thurston MO, McCabe DP, Farrar WB, Martin EW Jr: Intraoperative probe-directed immunodetection using a monoclonal antibody. Arch Surg 1986, 121:139|-1394.

295. Petty LR, Mojzisik CM, Hinkle GH, Ignaszewski J, Loesch J, Berens A, Thurston MO, Martin EW Jr: Radioimmunoguided surgery: a phase I/II study using iodine-I25 labeled to I7-IA IgG2A in patients with colorectal cancer. Antibody Immunoconjugates, Radiopharm I99I, 4:603-6II.

296. Colcher D, Keenan AM, Larson SM, Schlom J: Prolonged binding of a radiolabeled monoclonal antibody (B72.3) used for the in situ radioimmunodetection of human colon carcinoma xenografts. Cancer Res 1984, 44:5744-5749.

297. Sardi A, Siddiqi MA, Hinkle GH, Rousseau M, Gersman M, Hill T, Olsen J, Tuttle SE, Young D, Houchens D, Thurston M, Martin EW Jr: Localization by hand-held gamma probe of tumor labeled with antibody "cocktail". J Surg Res 1989, 47:227-234.

298. Martin EW Jr, Mojzisik CM, Hinkle GH, Sampsel J, Siddiqi M, Tuttle SE, Sickle-Santanello B, Colcher D, Thurston MO, Bell J, Farrar WB, Schlom J: Radioimmunoguided surgery using monoclonal antibody. Am J Surg 1988, 156:386-392.
299. Sickle-Santanello BJ, O'Dwyer PJ, Moizisik C, Tuttle SE, Hinkle GH, Rousseau M, Schlom J, Colcher D, Thurston MO, Nieroda C, Sardi A, Farrar WB, Minton JP, Martin EW Jr: Radioimmunoguided surgery using the monoclonal antibody B72.3 in colorectal tumors. Dis Colon Rectum 1987, 30:761-764.

300. Nieroda CA, Mojzisik C, Sardi A, Ferrara P, Hinkle G, Thurston MO, Martin EW Jr: Radioimmunoguided surgery in primary colon cancer. Cancer Detect Prev 1990, 1 4:65 I-656.

30I. Martin EW Jr, Carey LC: Second-look surgery for colorectal cancer. Ann Surg 1991, 21 4:321-325. discussion 326-327

302. Cohen AM, Martin EW Jr, Lavery I, Daly J, Sardi A, Aitken D, Bland K, Mojzisik C, Hinkle G: Radioimmunoguided surgery using iodine I 25 B72.3 in patients with colorectal cancer. Arch Surg 199I, I 26:349-352.

303. Bertoglio S, Benevento A, Percivale P, Cafiero F, Moresco L, Peressini $A$, Dionigi $R$, Badellino $F$ : Radioimmunoguided surgery benefits in carcinoembryonic antigen-directed second-look surgery in the asymptomatic patient after curative resection of colorectal cancer. Semin Surg Oncol 1998, 15:263-267.

304. Percivale P, Bertoglio S, Meszaros P, Schenone F, Gipponi M, Moresco L, Cosso M, Badellino F: Radioimmunoguided surgery with different iodine- 125 radiolabeled monoclonal antibodies in recurrent colorectal cancer. Semin Surg Oncol I998, I5:23I-234.

305. Renda A, lovino F, Capasso L, Ricciardelli L, Tammaro V, Acampa W: Radioimmunoguided surgery in colorectal cancer: a 6-year experience with four different technical solutions. Semin Surg Oncol 1998, 15:226-230.

306. Muxi A, Pons F, Vidal-Sicart S, Setoain FJ, Herranz R, Novell F, Fernandez RM, Trias M, Setoain J: Radioimmunoguided surgery of colorectal carcinoma with an IIIIn-labelled anti-TAG72 monoclonal antibody. Nucl Med Commun 1999, 20:123-130.

307. Collier BD, Abdel-Nabi H, Doerr RJ, Harwood SJ, Olsen J, Kaplan EH, Winzelberg GG, Grossman S], Krag DN, Mitchell EP: Immunoscintigraphy performed with In-III-labeled CYT-I03 in the management of colorectal cancer: comparison with CT. Radiology 1992, 185: 179-186.

308. Quinlan D, Davidson A, Gamponia E, Lindley J, Doshi H: Detection of tumor-associated glycoprotein-72 (TAG-72) in shortterm and long-term surviving colon cancer patients using a first- and second-generation monoclonal antibody. Proc Am Assoc Cancer Res 1991, 32:27I.

309. Arnold MW, Young DC, Hitchcock CL, Schneebaum S, Martin EW Jr: Radioimmunoguided surgery in primary colorectal carcinoma: an intraoperative prognostic tool and adjuvant to traditional staging. Am / Surg 1995, 170:315-3/8.

310. Bertsch DJ, Burak WE, Young DC, Arnold MW, Martin EW Jr: Radioimmunoguided surgery improves survival for patients recurrent colorectal cancer. Surg 1995, I | 8:634-638.

31I. Arnold MW, Young DC, Hitchcock CL, Barberá-Guillem E, Nieroda C, Martin EW Jr: Staging of colorectal cancer: biology vs. morphology. Dis Colon Rectum 1998, 41: I-6.

312. Avital S, Haddad R, Troitsa A, Kashtan H, Brazovsky E, Gitstein G, Skornick $Y$, Schneebaum S: Radioimmunoguided surgery for recurrent colorectal cancer manifested by isolated CEA elevation. Cancer 2000, 89:1692-1698.

313. Haddad R, Avital S, Troitsa A, Chen J, Baratz M, Brazovsky E Gitstein G, Kashtan H, Skornick Y, Schneebaum S: Benefits of radioimmunoguided surgery for pelvic recurrence. Eur J Surg Oncol 200I, 27:298-30I.

314. Schneebaum S, Troitsa A, Haddad R, Avital S, Kashtan H, Baratz M, Brazovsky E, Papo J, Skornick Y: Immunoguided lymph node dissection in colorectal cancer: a new challenge? World J Surg 200I, 25: I 495-I 498. discussion I499

315. Agnese DM, Abdessalam SF, Burak WE, Arnold MW, Soble D, Hinkle $\mathrm{GH}$, Young $\mathrm{D}$, Kazaeli MB, Martin EW Jr: Pilot study using a humanized CC49 monoclonal antibody (HuCC49 $\left.\Delta \mathrm{C}_{\mathrm{H}} 2\right)$ to localize recurrent colorectal carcinoma. I Surg Onc 2004, I I:197-202.

316. Lechner $P$, Lind $P$, Snyder $M$, Haushofer $\mathrm{H}$ : Probe-guided surgery for colorectal cancer. Recent Results Cancer Res 2000, I 57:273-280

317. Nastro P, Sodo M, Dodaro CA, Gargiulo S, Acampa W, Bracale U, Renda A: Intraoperative radiochromoguided mapping of sentinel lymph node in colon cancer. Tumori 2002, 88:352-353.

318. Trocha SD, Nora DT, Saha SS, Morton DL, Wiese D, Bilchik AJ: Combination probe and dye-directed lymphatic mapping 
detects micrometastases in early colorectal cancer. J Gastrointest Surg 2003, 7:340-345. discussion 345-346

319. Bilchik AJ, Nora DT, Sobin LH, Turner RR, Trocha S, Krasne D, Morton DL: Effect of lymphatic mapping on the new tumor-nodemetastasis classification for colorectal cancer. J Clin Oncol 2003, $21: 668-672$.

320. Patten LC, Berger DH, Rodriguez-Bigas M, Mansfield P, Delpassand E, Cleary KR, Fagan SP, Curley SA, Hunt KK, Feig BW: A prospective evaluation of radiocolloid and immunohistochemical staining in colon carcinoma lymphatic mapping. Cancer 2004, 100:2104-2109.

32I. Saha S, Dan AG, Berman B, Wiese D, Schochet E, Barber K, Choudhri S, Kaushal S, Ganatra B, Desai D, Nagaraju M, Mannam S: Lymphazurin $1 \%$ versus $99 \mathrm{mT}$ c sulfur colloid for lymphatic mapping in colorectal tumors: a comparative analysis. Ann Surg Oncol 2004, I I:2 I-26.

322. Bembenek A, Rau B, Moesta T, Markwardt J, Ulmer C, Gretschel S, Schneider U, Slisow W, Schlag PM: Sentinel lymph node biopsy in rectal cancer - not yet ready for routine clinical use. Surgery 2004, 135:498-505. discussion 506-507

323. Lim SJ, Feig BW, Wang H, Hunt KK, Rodriguez-Bigas MA, Skibber JM, Ellis V, Cleary K, Chang GJ: Sentinel lymph node evaluation does not improve staging accuracy in colon cancer. Ann Surg Oncol 2007, |5:46-5I.

324. Bembenek A, Gretschel S, Schlag PM: Sentinel lymph node biopsy for gastrointestinal cancers. J Surg Oncol 2007, 96:342-352.

325. Keshtgar MR, Amin A, Taylor I, Ell PJ: The sentinel node in anal carcinoma. Eur J Surg Oncol 200I, 27: II3-II4.

326. Rabbitt P, Pathma-Nathan N, Collinson T, Hewett P, Rieger N: Sentinel lymph node biopsy for squamous cell carcinoma of the anal canal. ANZ J Surg 2002, 72:65I-654

327. Mistrangelo M, Mobiglia A, Mussa B, Bellò M, Pelosi E, Goss M, Bosso MC, Moro F, Sandrucci S: The sentinel node in anal carcinoma. Tumori 2002, 88:S5I-S52.

328. Péley G, Farkas E, Sinkovics I, Kovács T, Keresztes S, Orosz Z, Köves I: Inguinal sentinel lymph node biopsy for staging anal cancer. Scand J Surg 2002, 9 1:336-338.

329. Perera D, Pathma-Nathan N, Rabbitt P, Hewett P, Rieger N: Sentinel node biopsy for squamous-cell carcinoma of the anus and anal margin. Dis Colon Rectum 2003, 46:1027-1029. discussion 1030-1031

330. Ulmer C, Bembenek A, Gretschel S, Markwardt J, Koswig S, Slisow W, Schneider U, Schlag PM: Sentinel node biopsy in anal cancer - a promising strategy to individualize therapy. Onkologie 2003, 26:456-460

33I. Bobin JY, Gérard JP, Chapet O, Romestaing P, Isaac S: [Lymphatic mapping and inguinal sentinel lymph node biopsy in anal canal cancers to avoid prophylactic inguinal irradiation]. Cancer Radiother 2003, 7(Suppl I):85s-90s. [French]

332. Damin DC, Rosito MA, Gus P, Spiro BL, Amaral BB, Meurer L, Cartel A, Schwartsmann G: Sentinel lymph node procedure in patients with epidermoid carcinoma of the anal canal: early experience. Dis Colon Rectum 2003, 46:1032-1037.

333. Ulmer C, Bembenek A, Gretschel S, Markwardt J, Koswig S, Schneider $U$, Schlag PM: Refined staging by sentinel lymph node biopsy to individualize therapy in anal cancer. Ann Surg Oncol 2004, I I (3 Suppl):259S-262S.

334. Mistrangelo M, Mobiglia A, Bellò M, Beltramo G, Cassoni P, Mussa A: [The technique of sentinel lymph nodes in patients with anus neoplasm]. Supp/ Tumori 2005, 4:S32-S33. [Italian]

335. Damin DC, Rosito MA, Schwartsmann G: Sentinel lymph node in carcinoma of the anal canal: a review. Eur J Surg Oncol 2006 32:247-252.

336. Gretschel S, Warnick P, Bembenek A, Dresel S, Koswig S, String A, Hünerbein M, Schlag PM: Lymphatic mapping and sentinel lymph node biopsy in epidermoid carcinoma of the anal canal. Eur J Surg Oncol 2008, 34:890-894.

337. Mistrangelo M, Bellò M, Mobiglia A, Beltramo G, Cassoni P, Milanesi E, Cornaglia S, Pelosi E, Giunta F, Sandrucci S, Mussa A: Feasibility of the sentinel node biopsy in anal cancer. $Q$ J Nucl Med Mol Imaging 2008 in press.

338. Kitagawa $Y$, Ohgami M, Fujii H, Mukai M, Kubota T, Ando N, Watanabe M, Otani Y, Ozawa S, Hasegawa H, Furukawa T, Matsuda J, Kumai K, Ikeda T, Kubo A, Kitajima M: Laparoscopic detection of sentinel lymph nodes in gastrointestinal cancer: a novel and min- imally invasive approach. Ann Surg Oncol 200I, 8(9 Suppl):86S-89S.

339. Kitagawa $Y$, Kitajima M: Gastrointestinal cancer and sentinel node navigation surgery. J Surg Oncol 2002, 79:188-193.

340. Tajima T, Tokuda Y, Yasuda S: [The sentinel navigation surgery: current review and future perspective]. Nippon Rinsho 2002, 60:197-205.

34I. Kitagawa Y, Fujii H, Mukai M, Kubo A, Kitajima M: Current status and future prospects of sentinel node navigational surgery for gastrointestinal cancers. Ann Surg Oncol 2004, I I:242S-244S.

342. Kitagawa $Y$, Kitajima M: Lymphatic mapping for upper gastrointestinal malignancies. Semin Oncol 2004, 31:409-414.

343. Takeuchi H, Kitagawa $Y$ : Sentinel node navigation surgery for esophageal cancer. Gen Thorac Cardiovasc Surg 2008, 56:393-396.

344. Kitagawa $Y$, Fujii $H$, Mukai $M$, Kubota $T$, Ando $N$, Watanabe $M$, Ohgami M, Otani Y, Ozawa S, Hasegawa H, Furukawa T, Kumai K, Ikeda T, Nakahara T, Kubo A, Kitajima M: The role of the sentinel lymph node in gastrointestinal cancer. Surg Clin North Am 2000, 80:1799-1809.

345. Yasuda S, Shimada H, Ogoshi K, Tanaka H, Kise Y, Kenmochi T, Soeda I, Nakamura K, Kato Y, Kijima H, Suzuki Y, Fujii H, Tajima T, Makuuchi $H$ : Preliminary study for sentinel lymph node identification with Tc-99m tin colloid in patients with esophageal or gastric cancer. Tokai J Exp Clin Med 200I, 26:15-18.

346. Yasuda S, Shimada H, Chino O, Tanaka H, Kenmochi T, Takechi M, Nabeshima K, Okamoto Y, Kato Y, Kijima H, Suzuki Y, Ogoshi K, Tajima T, Makuuchi H: Sentinel lymph node detection with Tc$99 \mathrm{~m}$ tin colloids in patients with esophagogastric cancer. Jpn I Clin Oncol 2003, 33:68-72.

347. Kato H, Miyazaki T, Nakajima M, Takita J, Sohda M, Fukai Y, Masuda N, Fukuchi M, Manda R, Ojima H, Tsukada K, Asao T, Kuwano H, Oriuchi N, Endo K: Sentinel lymph nodes with technetium-99m colloidal rhenium sulfide in patients with esophageal carcinoma. Cancer 2003, 98:932-939.

348. Yasuda S, Shimada H, Chino O, Nishi T, Takechi M, Makuuchi H: [Sentinel lymph node concept in patients with esophageal cancer]. Gan To Kagaku Ryoho 2005, 32:877-88I. [Japanese]

349. Arima H, Natsugoe S, Uenosono $\mathrm{Y}$, Arigami T, Ehi K, Yanagita S, Higashi H, Ishigami S, Hokita S, Aikou T: Area of nodal metastasis and radioisotope uptake in sentinel nodes of upper gastrointestinal cancer. J Surg Res 2006, 135:250-254.

350. Kosugi S, Nakagawa S, Kanda T, Odano I, Yajima K, Kaneko K, Ohashi $\mathrm{M}$, Hatakeyama K: Radio-guided sentinel node mapping in patients with superficial esophageal carcinoma: feasibility study. Minim Invasive Ther Allied Technol 2007, 16:181-186.

35I. Lamb PJ, Griffin SM, Burt AD, Lloyd J, Karat D, Hayes N: Sentinel node biopsy to evaluate the metastatic dissemination of oesophageal adenocarcinoma. Br J Surg 2005, 92:60-67.

352. Burian M, Stein HJ, Sendler A, Piert M, Nährig J, Feith M, Siewert JR: Sentinel node detection in Barrett's and cardia cancer. Ann Surg Oncol 2004, I I (3 suppl):255S-258S.

353. Bonenkamp JJ, Hermans J, Sasako M, Velde CJ van de, Welvaart $K$, Songun I, Meyer S, Plukker JT, Van Elk P, Obertop H, Gouma DJ, van Lanschot J], Taat CW, de Graaf PW, von Meyenfeldt MF, Tilanus H, Dutch Gastric Cancer Group: Extended lymph-node dissection for gastric cancer. N Engl] Med 1999, 340:908-9|4.

354. Cuschieri A, Weeden S, Fielding J, Bancewicz J, Craven J, Joypaul V, Sydes $M$, Fayers $P$ : Patient survival after $D 1$ and $D 2$ resections for gastric cancer: long-term results of the MRC randomized surgical trial. Surgical Co-operative Group. Br J Cancer 1999, 79: $1522-1530$.

355. Fass J, Hungs M, Reineke T, Nachtkamp J, Schumpelick V: Prognostic improvement by $R \mathbf{l}$ and $R 2$ lymphadenectomy in stomach carcinoma. Chirurg 1994, 65:867-872.

356. Siewert JR, Böttcher K, Roder JD, Busch R, Hermanek P, Meyer HJ: Prognostic relevance of systematic lymph node dissection in gastric carcinoma. German Gastric Carcinoma Study Group. BrJ Surg 1993, 80:1015-1018.

357. Sasako M, McCulloch P, Kinoshita T, Maruyama K: New method to evaluate the therapeutic value of lymph node dissection for gastric cancer. Br J Surg 1995, 82:346-35I.

358. Aikou T, Higashi H, Natsugoe S, Hokita S, Baba M, Tako S: Can sentinel node navigation surgery reduce the extent of lymph node dissection in gastric cancer? Ann Surg Oncol 2001, 8(9 Suppl):90S-93S. 
359. Kitagawa Y, Fujii H, Mukai M, Kubota T, Otani Y, Kitajima M: Radioguided sentinel node detection for gastric cancer. $\mathrm{Br} J$ Surg 2002, 89:604-608.

360. Hayashi H, Ochiai T, Mori M, Karube T, Suzuki T, Gunji Y, Hori S, Akutsu N, Matsubara H, Shimada H: Sentinel lymph node mapping for gastric cancer using a dual procedure with dye- and gamma probe-guided techniques. I Am Coll Surg 2003, 196:68-74.

36I. Tonouchi H, Mohri Y, Tanaka K, Konishi N, Ohmori Y, Kobayashi M, Watanabe Y, Matsumura K, Takeda K, Kusunoki M: Lymphatic mapping and sentinel node biopsy during laparoscopic gastrectomy for early cancer. Dig Surg 2003, 20:42 I-427.

362. Gretschel S, Bembenek A, Ulmer Ch, Hünerbein M, Markwardt J, Schneider U, Schlag PM: [Lymphatic mapping and sentinel lymph node biopsy in gastric cancer]. Chirurg 2003, 74: I32-138. [German]

363. Asao T, Kuwano H, Ide M, Hirayama I, Nakamura J, Endo K: Intraoperative location of small gastrointestinal cancers with a handheld gamma probe. Surg Endosc 2003, I7:1216-1217.

364. Uenosono $Y$, Natsugoe S, Higashi H, Ehi K, Miyazono F, Ishigami S, Hokita S, Aikou T: Evaluation of colloid size for sentinel nodes detection using radioisotope in early gastric cancer. Cancer Lett 2003, 200:19-24.

365. Kim MC, Jung GJ, Lee JH, Choi SR, Kang DY, Roh MS, Jeong JS: Sentinel lymph node biopsy with $99 \mathrm{mTC}$ tin-colloid in patients with gastric carcinoma. Hepatogastroenterology 2003, 50(Suppl 2):2|4-2|5.

366. Kim MC, Kim HH, Jung G], Lee JH, Choi SR, Kang DY, Roh MS, Jeong JS: Lymphatic mapping and sentinel node biopsy using $99 \mathrm{mTc}$ tin colloid in gastric cancer. Ann Surg 2004, 239:383-387.

367. Tanaka K, Tonouchi H, Kobayashi M, Konishi N, Ohmori Y, Mohri Y, Kusunoki M: Laparoscopically assisted total gastrectomy with sentinel node biopsy for early gastric cancer: preliminary results. Am Surg 2004, 70:976-981.

368. Gretschel S, Bembenek A, Ulmer Ch, Hünerbein M, Markwardt J, Schneider U, Schlag PM: Prediction of gastric cancer lymph node status by sentinel lymph node biopsy and the Maruyama computer model. Eur J Surg Oncol 2005, 3 I:393-400.

369. Uenosono Y, Natsugoe S, Ehi K, Arigami T, Hokita S, Aikou T. Detection of sentinel nodes and micrometastases using radioisotope navigation and immunohistochemistry in patients with gastric cancer. BrJ Surg 2005, 92:886-889.

370. Cheng LY, Chen XD, Zhang YX, Feng XD: [Clinical significance of sentinel lymph node detection by combining the dyedirected and radioguided methods in gastric cancer]. Zhonghua Wai Ke Za Zhi 2005, 43:569-572. [Chinese]

37I. Tonouchi H, Mohri Y, Tanaka K, Kobayashi M, Ohmori Y, Kusunoki $M$ : Laparoscopic lymphatic mapping and sentinel node biopsies for early-stage gastric cancer: the cause of false negativity. World J Surg 2005, 29:4 I8-42I.

372. Zulfikaroglu B, Koc M, Ozmen MM, Kucuk NO, Ozalp N, Aras G: Intraoperative lymphatic mapping and sentinel lymph node biopsy using radioactive tracer in gastric cancer. Surgery 2005 I 38:899-904.

373. Mura G, Vagliasindi A, Framarini M, Mazza P, Solfrini G, Verdecchia GM: The sentinel node biopsy in early gastric cancer: a preliminary study. Langenbecks Arch Surg 2006, 39 I: I I3-I I 7.

374. Mochiki E, Kuwano H, Kamiyama Y, Aihara R, Nakabayashi T, Katoh $H$, Asao T, Oriuchi N, Endo K: Sentinel lymph node mapping with technetium-99m colloidal rhenium sulfide in patients with gastric carcinoma. Am J Surg 2006, I 9 I:465-469.

375. Ichikura T, Chochi K, Sugasawa H, Yaguchi Y, Sakamoto N, Takahata $\mathrm{R}$, Kosuda S, Mochizuki H: Individualized surgery for early gastric cancer guided by sentinel node biopsy. Surgery 2006, I39:50|-507.

376. Lee JH, Ryu KW, Kim CG, Kim SK, Lee JS, Kook MC, Choi IJ, Kim YW, Chang HJ, Bae JM: Sentinel node biopsy using dye and isotope double tracers in early gastric cancer. Ann Surg Oncol 2006, I 3: I 168-I I74

377. Saikawa Y, Otani Y, Kitagawa Y, Yoshida M, Wada N, Kubota T, Kumai K, Sugino Y, Mukai M, Kameyama K, Kubo A, Kitajima M Interim results of sentinel node biopsy during laparoscopic gastrectomy: possible role in function-preserving surgery for early cancer. World J Surg 2006, 30:1962-1968.
378. Tonouchi H, Mohri Y, Kobayashi M, Tanaka K, Ohi M, Kusunoki M: Laparoscopy-assisted distal gastrectomy with laparoscopic sentinel lymph node biopsy after endoscopic mucosal resection for early gastric cancer. Surg Endosc 2007, 2 | : | 289- | 293.

379. Gretschel S, Bembenek A, Hünerbein M, Dresel S, Schneider W, Schlag PM: Efficacy of different technical procedures for sentinel lymph node biopsy in gastric cancer staging. Ann Surg Oncol 2007, I 4:2028-2035.

380. Ishigami S, Natsugoe S, Uenosono Y, Yanagita S, Matsumoto M, Okumura H, Uchikado Y, Arigami T, Arima H, Setoyama T, Aikou T: Usefulness of sentinel node biopsy in laparoscopic partial gastrectomy for early gastric cancer. Hepatogastroenterology 2007, 54:2164-2166.

38I. Lee JH, Ryu KW, Kook MC, Lee JY, Kim CG, Choi IJ, Kim SK, Jang S, Park SR, Kim YW, Nam BH, Bae JM: Feasibility of laparoscopic sentinel basin dissection for limited resection in early gastric cancer. J Surg Oncol 2008, 98:331-335.

382. Lee YJ, Ha WS, Park ST, Choi SK, Hong SC, Park JW: Which biopsy method is more suitable between a basin dissection and pick-up biopsy for sentinel nodes in laparoscopic sentinelnode navigation surgery (LSNNS) for gastric cancer? J Laparoendosc Adv Surg Tech A 2008, I 8:357-363.

383. Xu G, Zhang M, Liu B, Li Z, Lin B, Xu X, Jin M, Li J, Wu J, Dong Z: Radioimmunoguided surgery in gastric cancer using $|3|-\mid$ labeled monoclonal antibody 3HII. Semin Surg Oncol 1994, 10:88-94.

384. Liu BG, Xu GW, Zhang MY: [A clinical trial of radioimmunoguided surgery for gastric cancer]. Zhonghua Zhong Liu Za Zhi I994, 16:284-287. [Chinese]

385. Lucisano $E$, Bertoglio S: Role of radioimmunoguided surgery using iodine- I 25-labeled B72.3 monoclonal antibody in gastric cancer surgery. Semin Surg Oncol 1998, I 5:212-2I4.

386. Mussa A, Sandrucci S, Mobiglia A, Baccega M, Mussa B, De Filippi PG: Role of radioimmunolocalization in the staging of gastric carcinoma. Semin Surg Oncol 1998, I 5:209-2II.

387. Wang C, Wang Y, Su X, Lin B, Xu X, Zhang M, Li J, Xu G: [lodineI 25 labeled monoclonal antibody $3 \mathrm{HI}$ : in radioimmunoguided surgery for primary gastric cancer]. Zhonghua Wai Ke $\mathrm{Za}$ Zhi 2000, 38:507-509. [Chinese]

388. LaValle GJ, Martinez DA, Sobel D, DeYoung B, Martin EW Jr: Assessment of disseminated pancreatic cancer: a comparison of traditional exploratory laparotomy and radioimmunoguided surgery. Surgery 1997, I 22:867-87I. discussion 87I-873

389. Dunne AA, Budach VG, Wagner W, Werner J: Management of NO neck in head and neck cancer: current controversies. Onkologie 2004, 27:363-367.

390. Alex JC, Krag DN: Gamma-probe guided resection of radiolabeled primary lymph nodes. Surg Oncol Clin N Am 1996, 5:33-4I.

39I. Chikamatsu K, Kamada H, Ninomiya H, Takahashi K, Sakurai T, Oriuchi N, Furuya N: A preliminary study on sentinel lymph node biopsy: feasibility and predictive ability in oral cavity cancer. Ann Nucl Med 2004, I 8:257-262.

392. Civantos F, Zitsch R, Bared A: Sentinel Node Biopsy in Oral Squamous Cell Carcinoma. Journal of Surgical Oncology 2007, 96:330-336.

393. Stoeckli SJ: Sentinel node biopsy for oral and oropharyngeal squamous cell carcinoma of the head and neck. Laryngoscope 2007, I | 7: |539-|55 |

394. Werner JA, Dünne AA, Ramaswamy A, Brandt D, Külkens C, Folz BJ, Moll $R$, Lippert BM: [The sentinel node concept in head and neck squamous cell carcinoma - a critical analysis in 100 patients]. Laryngorhinootologie 2002, 81:31-39. [German]

395. Ross GL, Soutar DS, MacDonald DG, Shoaib T, Camilleri I, Roberton AG, Sorensen JA, Thomsen J, Grupe P, Alvarez J, Barbier L, Santamaria J, Poli T, Massarelli O, Sesenna E, Kovacs AF, Grunwald F, Barzan L, Sulfaro S, Alberti F: Sentinel node biopsy in head and neck cancer: preliminary results of a multicenter trial. Ann Surg Oncol 2004, I I:690-696.

396. Vigili MG, Tartaglione G, Rahimi S, Mafera B, Pagan M: Lymphoscintigraphy and radioguided sentinel node biopsy in oral cavity sqaumous cell carcinoma: same day protocol. Eur Arch Otorhinolaryngol 2007, 264: I63-I67.

397. Tomifuji M, Shiotani A, Fujii H, Araki K, Saito K, Inagaki K, Mukai M, Kitagawa $Y$, Ogawa K: Sentinel node concept in clinically no 
laryngeal and hypopharyngeal cancer. Ann Surg Oncol 2008, I 5:2568-2575

398. Atula T, Shoaib T, Ross GL, Gray HW, Soutar DS: How many sentinel nodes should be harvested in oral squamous cell carcinoma? Eur Arch Otorhinolaryngol 2007, 265(SuppI I):S19-S23.

399. Jesse RH, Ballantyne AJ, Larson D: Radical or modified radical neck dissection: a therapeutic dilemma. Am J Surg 1978, 136:516-519.

400. National Comprehensive Cancer Network (NCCN) Clinical Guidelines in Oncology for Head and Neck Cancers (V.2.2008) [http://www.nccn.org/professionals/physician gls/PDF/ head-and-neck.pdf]

40I. Kovács AF, Landes CA, Hamscho N, Risse JH, Berner U, Menzel C: Sentinel node biopsy as staging tool in a multi-modality treatment approach to cancer of the oral cavity and oropharynx. Otolaryngol Head Neck Sug 2005, 132:570-576.

402. Matsuzuka T, Kano M, Ohtani I, Miura T, Shishido F, Omori K: Impact of sentinel node navigation technique for carcinoma of the tongue with cervical node metastases. Auris Nasus Larynx 2005, 32:59-63.

403. Mozzillo N, Chiesa F, Caraco C, Botti G, Lastoria S, Longo F, lonna F: Therapeutic implications of sentinel lymph node bipsy in the staging of oral cancer. Ann Surg Oncol 2004, I I(3 Suppl):263S-266S.

404. Kovács AF: Head and neck squamous cell carcinoma: sentinel node or selective neck dissection. Surg Oncol Clin N Am 2007 , 16:8I-100.

405. Byers RM, Weber RS, Andrews T, McGill D, Kare R, Wolf P: Frequency and therapeutic implications of skip metastases in neck from squamous carcinoma of the oral tongue. Head Neck 1997, 19:14-19.

406. Argenzio V, Rambaldi PF, lorio G, Cuccurullo V, Cascini G, Argenzio $G$, Mansi L: Radioimmunoguided surgery in squamous cell carcinoma. J Am Soc Plas Surg 1999, 103:749-75I.

407. Kaplan E, Yashiro T, Salti G: Primary hyperaparathyroidism in the 1990s: choice of surgical procedures for this disease. Ann Surg 1992, 215:300-317.

408. Ubhi CS, Hardy JG, Pegg CA: Mediastinal parathyroid adenoma: a new method of localization. Br J Surg 1984, 71:859-860.

409. Martinez DA, King DR, Romshe C, Lozano RA, Morris JD, O'Dorisio MS, Martin E Jr: Intraoperative identification of parathyroid gland pathology: a new approach. J Pediatr Surg 1995, 30:1306-1309.

410. Norman J, Chheda : Minimally invasive parathyroidectomy facilitated by intraoperative nuclear mapping. Surgery 1997 I 22:998-1003. discussion 1003-1004

4II. Murphy C, Norman J: The $20 \%$ rule: a simple, instantaneous radioactivity measurement defines cure and allows elimination of frozen sections and hormone assays during parathyroidectomy. Surgery 1999, I 26: 1023-1028. discussion 1028-1029

4I2. Flynn MB, Bumpous JM, Schill K, McMasters KM: Minimally invasive radioguided parathyroidectomy. J Am Coll Surg 2000, I 9 I:24-31.

413. Goldstein RE, Blevins L, Delbeke D, Martin WH: Effect of minimally invasive radioguided parathyroidectomy on efficacy, length of stay, and costs in the management of primary hyperparathyroidism. Ann Surg 2000, 231:732-742.

414. Norman J, Jaffray CE, Chheda $\mathrm{H}$ : The false-positive parathyroid sestamibi: a real or perceived problem and a case for radioguided parathyroidectomy. Ann Surg 2000, 23 I:31-37.

415. Dackiw AP, Sussman JJ, Fritsche HA Jr, Delpassand ES, Stanford P, Hoff A, Gagel RF, Evans DB, Lee JE: Relative contributions of technetium Tc $99 \mathrm{~m}$ sestamibi scintigraphy, intraoperative gamma probe detection, and the rapid parathyroid hormone assay to the surgical management of hyperparathyroidism. Arch Surg 2000, 135:550-555. discussion 555-557

416. Casara D, Rubello D, Pelizzo MR, Shapiro B: Clinical role of $99 \mathrm{mTcO} 4 / \mathrm{MIBI}$ scan, ultrasound and intra-operative gamma probe in the performance of unilateral and minimally invasive surgery in primary hyperparathyroidism. Eur I Nucl Med 200I, 28:135|-1359.

417. McGreal G, Winter DC, Sookhai S, Evoy D, Ryan M, O'Sullivan GC, Redmond HP: Minimally invasive, radioguided surgery for primary hyperparathyroidism. Ann Surg Oncol 200I, 8:856-860.

418. Rubello D, Casara D, Saladini G, Piotto A, Pagetta C, Pelizzo MR: $99 \mathrm{mTC}-\mathrm{MIBI}$ radio-guided surgery in primary hyperparathy roidism: a prospective study of $\mathbf{I} 28$ patients. Tumori 2002 88:S63-S65.

419. Rubello D, Piotto, Casara D, Muzzio PC, Shapiro B, Pelizzo MR: Role of gamma probes in performing minimally invasive parathyroidectomy in patients with primary hyperparathyroidism: optimization of preoperative and intraoperative procedures. Eur J Endocrinol 2003, 149:7-I5.

420. Rubello D, Casara D, Giannini S, Piotto A, De Carlo E, Muzzio PC, Pelizzo MR: Importance of radio-guided minimally invasive parathyroidectomy using hand-held gamma probe and low (99m)Tc-MIBI dose. Technical considerations and long-term clinical results. Q J Nucl Med 2003, 47:129-138.

42I. Rubello D, Pelizzo MR, Boni G, Schiavo R, Vaggelli L, Villa G, Sandrucci S, Piotto A, Manca G, Marini P, Mariani G: Radioguided surgery of primary hyperparathyroidism using the low-dose $99 \mathrm{mTc}$ sestamibi protocol: multiinstitutional experience from the Italian Study Group on Radioguided Surgery and Immunoscintigraphy (GISCRIS). J Nucl Med 2005, 46:220-226.

422. Rubello D, Giannini S, Martini C, Piotto A, Rampin L, Fanti S, Armigliato M, Nardi A, Carpi A, Mariani G, Gross MD, Pelizzo MR: Minimally invasive radio-guided parathyroidectomy. Biomed Pharmacother 2006, 60:134-138.

423. Rubello D, Mariani G, Al-Nahhas A, Pelizzo MR, Italian Study Group on Radioguided Surgery and Immunoscintigraphy (GISCRIS): Minimally invasive radio-guided parathyroidectomy: long-term results with the "low 99mTc-sestamibi protocol". Nucl Med Commun 2006, 27:709-7।3.

424. Rubello D, Mariani G, Pelizzo MR, Italian Study Group of Radioguided Surgery and ImmunoScintigraphy: Minimally invasive radioguided parathyroidectomy on a group of $\mathbf{4 5 2}$ primary hyperparathyroid patients: refinement of preoperative imaging and intraoperative procedure. Nuklearmedizin 2007, 46:85-92.

425. Shabtai M, Ben-Haim M, Muntz Y, Vered I, Rosin D, Kuriansky J, Zmora O, Olchovski D, Ayalon A, Zwas ST: I 40 consecutive cases of minimally invasive, radio-guided parathyroidectomy: lessons learned and long-term results. Surg Endosc 2003, |7:688-69|.

426. Bekiş R, Aydin A, Taşci C, Atila K, Koçdor MA, Sevinç A, Canda S, Harmancioğlu $\mathrm{O}$, Durak $\mathrm{H}$ : The role of gamma probe activity counts in minimally invasive parathyroidectomy. Preliminary results. Nuklearmedizin 2004, 43:190-194.

427. Goldstein RE, Billheimer D, Martin WH, Richards K: Sestamibi scanning and minimally invasive radioguided parathyroidectomy without intraoperative parathyroid hormone measurement. Ann Surg 2003, 237:722-730. discussion 730-73I

428. Chen H, Pruhs Z, Starling JR, Mack E: Intraoperative parathyroid hormone testing improves cure rates in patients undergoing minimally invasive parathyroidectomy. Surgery 2005, 138:583-587. discussion 587-590

429. Caudle AS, Brier SE, Calvo BF, Kim HJ, Meyers MO, Ollila DW: Experienced radio-guided surgery teams can successfully perform minimally invasive radio-guided parathyroidectomy without intraoperative parathyroid hormone assays. Am Surg 2006, 72:785-789. discussion 790

430. Norman J, Politz D: Measuring individual parathyroid gland hormone production in real-time during radioguided parathyroidectomy. Experience in over $\mathbf{8 0 0 0}$ operations. Minerva Endocrinol 2008, 33:147-157.

43I. Lal $A$, Chen $H$ : The negative sestamibi scan: is a minimally invasive parathyroidectomy still possible? Ann Surg Oncol 2007, 1 4:2363-2366.

432. Rossi HL, Ali A, Prinz RA: Intraoperative sestamibi scanning in reoperative parathyroidectomy. Surgery 2000, 128:744-750.

433. Navarra G, Ascanelli S, Feggi L, Carcoforo P, Turini A: Radio-guided surgery in recurrent renal hyperparathyroidism: report of a case. Head Neck 2000, 22:618-620.

434. Navarra G, Feggi L, Ascanelli S, Turini A, Carcoforo P, De Paoli E, Pozza $E$ : Role of radio-guided surgery in recurrent secondary hyperparathyroidism. Nephron 2001, 88:36-38.

435. Geissler B, Gröber S, Zügel N, Lindemann F: [Radio-guided parathyroidectomy: successful intraoperative parathyroid localization diagnosis with $99 \mathrm{mTc}$-sestamibi in primary and recurrent hyperparathyroidism]. Chirurg 2001, 72:1।79-II85. [German] 
436. Chen $\mathrm{H}$, Mack E, Starling JR: Radioguided parathyroidectomy is equally effective for both adenomatous and hyperplastic glands. Ann Surg 2003, 238:332-337. discussion 337-338

437. Nichol PF, Mack E, Bianco J, Hayman A, Starling JR, Chen H: Radioguided parathyroidectomy in patients with secondary and tertiary hyperparathyroidism. Surgery 2003, 134:7/3-717. discussion 7|7-719

438. Kitagawa W, Shimizu K, Akasu H, Tanaka S: Radioguided parathyroidectomy for renal hyperparathyroidism. Med Sci Monit 2003, 9:CS9-CSI2.

439. Ogi S, Fukumitsu N, Uchiyama M, Mori Y, Takeyama H: The usefulness of radio-guided surgery in secondary hyperparathyroidism. Ann Nucl Med 2004, 1 8:69-7I.

440. Takeyama H, Shioya H, Mori Y, Ogi S, Yamamoto H, Kato N, Kinoshita S, Yoshida K, Uchida K, Yamazaki Y: Usefulness of radioguided surgery using technetium-99m methoxyisobutylisonitrile for primary and secondary hyperparathyroidism. World J Surg 2004, 28:576-582.

44I. Kebebew E, Arici C, Duh QY, Clark OH: Localization and reoperation results for persistent and recurrent parathyroid carcinoma. Arch Surg 200I, 136:878-885.

442. Placzkowski K, Christian R, Chen H: Radioguided parathyroidectomy for recurrent parathyroid cancer. Clin Nucl Med 2007, 32:358-360.

443. Carcoforo P, Feggi L, Trasforini G, Lanzara S, Sortini D, Zulian V, Pansini GC, Degli Uberti E, Liboni A: Use of preoperative lymphoscintigraphy and intraoperative gamma probe detection for identification of the sentinel lymph node in patients with papillary thyroid carcinoma. Eur J Sug Oncol 2007, 33: I 075- 1080.

444. Mazzaferri EL, Kloos RT: Clinical review 128: Current approaches to primary therapy for papillary and follicular thyroid cancer. J Clin Endocrinol Metab 200I, 86: I 447-I 463.

445. Salvatori M, Rufini V, Reale F, Gajate AM, Maussier ML, Revelli L, Troncone L, Ardito G: Radio-guided surgery for lymph node recurrences of differentiated thyroid cancer. World J Surg 2003, 27:770-775.

446. Rubello D, Salvatori M, Ardito G, Al-Nahhas A, Gross M, Muzzio P, Pelizzo M: lodine- $13 \mathrm{I}$ radio-guided surgery in differentiated thyroid cancer: outcome on $3 I$ patients and review of the literature. Biomed Pharmacother 2007, 61:477-81.

447. Negele T, Meisetschläger G, Brückner T, Scheidhauer K, Schwaiger $M$, Vogelsang $H$ : Radio-guided surgery for persistent differentiated papillary thyroid cancer: case presentations and review of the literature. Langenbecks Arch Surg 2006, 391:178-186.

448. Robbins R, Schlumberger M: The evolving role of $|3|$ | for the treatment of differentiated thyroid carcinoma. I Nucl Med 2005, 46:28S-37S.

449. Vassilopoulou-Sellin R, Schultz PN, Haynie TP: Clinical outcomes of patients with papillary thyroid carcinoma who have recurrence after initial radioactive iodine therapy. Cancer 1996, 78:273-282.

450. Vassilopoulou-Sellin R: Long-term outcomes of children with papillary thyroid cancer. Surgery 200I, I 29:769.

45I. Haugen BR: Management of the patient with progressive radioiodine non-responsive disease. Semin Surg Oncol 1999, 16:34-4I.

452. Morris AC Jr, Barclay TR, Tanida R, Nemcek JV: A miniaturized probe for detecting radioactivity at thyroid surgery. Phys Med Biol 1971, 16:397-404.

453. Travagli JP, Cailleux AF, Ricard M, Baudin E, Caillou B, Parmentier C: Combination of radioiodine $(13 I I)$ and probe-guided surgery for persistent or recurrent thyroid carcinoma. J Clin Endocrinol Metab 1998, 83:2675-2680.

454. Scurry WC, Lamarre E, Stack B: Radioguided neck dissection in recurrent metastatic papillary thyroid carcinoma. Am J Otolaryngol 2006, 27:6I-63.

455. Gallowitsch H, Fellinger J, Mikosch P, Kresnik E, Lind P: Gammaprobe guided resection of a lymph node metastasis with I123 in papillary thyroid carcinoma. Clin Nucl Med 1997, 22:591-592.

456. Khandelwal S, Sener S, Purdy L, PerIman R: I- I 23-Guided excision of metastatic papillary thyroid cancer. J Surg Onc 2007, 96:173-175

457. Shimotake T, Tsuda T, Aoi S, Fumino S, Iwai N: lodine I 23 metaiodobenzylguanidine radio-guided navigation surgery for recurrent medullary thyroid carcinoma in a girl with multi- ple endocrine neoplasia type 2B. I Pediatr Surg 2005, 40:1643-1646.

458. Alam MS, Kasai K, Miisaki T, Miyamato S, Iwata M, lida Y, Knishi J: Diagnostic value of technetium-99m methoxyisobutyl isonitrile $(99 \mathrm{mTc}-\mathrm{MIBI})$ scintigraphy in detecting thyroid cancer metastases: a critical evaluation. Thyroid 1998, 8:1091-I 100.

459. Ugur O, Kostakğlu L, Güler N, Caner B, Uysal U, Elahi N, Haliloğlu M, Yüksel D, Aras T, Bayhan H, Bekdik C: Comparison of $99 \mathrm{mTc}(\mathrm{V})$-DMSA, $20 \mathrm{ITI}$ and $99 \mathrm{mTc}$-MIBI imaging in the follow-up of patients with medullary carcinoma of the thyroid. Eur J Nucl Med 1996, 23:1367-1371.

460. Chen YK, Liu FY, Yen RF, Kao CH: Compare FDG-PET and Tc$99 \mathrm{~m}$ tetrafosmin SPECT to detect metastatic thyroid carcinoma. Acad Radiol 2003, 10:835-839.

46I. Rubello D, Pelizzo MR, Casara D, Piotto A, Toniato A, Fig L, Gross M: Radio-guided surgery for non-13I I-avid thyroid cancer. Thyroid 2006, 16:1105-IIII.

462. Rubello D, Piotto A, Pagetta C, Pelizzo MR, Casara D: (99m)TcMIBI radio-guided surgery for recurrent thyroid carcinoma: technical feasibility and procedure, and preliminary clinical results. Eur J Nucl Med Mol Imaging 2002, 29: 1 20I- 205

463. Adams S, Acker P, Lorenz M, Staib-Sebler E, Hör G: Radioisotopeguided surgery in patients with pheochromocytoma and recurrent medullary thyroid carcinoma: a comparison of preoperative and intraoperative tumor localization with histopathologic findings. Cancer 200I, 92:263-270.

464. Stokkel M, Verkooijen , Smit J: Indium-I I I octreotide scintigraphy for the detection of non-functioning metastases from differentiated thyroid cancer: diagnostic and prognostic value. Eur J Nucl Med Mol Imaging 2004, 3 I:950-957.

465. Peltier P, Curtet C, Chatal JF, Le Doussal JM, Daniel G, Aillet G Gruaz-Guyon A, Barbet J, Delaage M: Radioimmunodetection of medullary thyroid cancer using a bispecific anti-CEA/antiindium-DTPA antibody and an indium-III-labeled DTPA dimer. J Nucl Med 1993, 34:1267-1273.

466. Barbet J, Peltier P, Bardet S, Vuillez JP, Bachelot I, Denet S, Olivier P, Leccia F, Corcuff B, Huglo D, Proye C, Rouvier E, Meyer P, Chatal JF: Radioimmunodetection of medullary thyroid carcinoma using indium-III bivalent hapten and anti-CEA $x$ antiDTPA-indium bispecific antibody. I Nucl Med 1998, 39: $1172-1178$.

467. de Labriolle-Vaylet C, Cattan P, Sarfati E, Wioland M, Billotey C, Brochériou $C$, Rouvier $E$, de Roquancourt $A$, Rostène $W$, Askienazy $S$, Barbet J, Milhaud G, Gruaz-Guyon A: Successful surgical removal of occult metastases of medullary thyroid carcinoma recurrences with the help of immunoscintigraphy and radioimmunoguided surgery. Clin Cancer Res 2000, 6:363-37I.

468. Meller B, Sahlmann C, Horstmann O, Gerl J, Baehre M, Meller J: Conventional gamma and high energy probe for radioguided dissection of metastases in a patient with recurrent thyroid carcinoma with (99m)Tc-MIBI and ( 1 8)F-FDG. Nuklearmedizin 2005, 44: N23-N25

469. Curtet C, Carlier T, Mirallié E, Bodet-Milin C, Rousseau C, Barbet J, Kraeber-Bodéré F: Prospective comparison of two gamma probes for intraoperative detection of 18F-FDG: in vitro assessment and clinical evaluation in differentiated thyroid cancer patients with iodine-negative recurrence. Eur J Nucl Med Mol Imaging 2007, 34: I556-1562.

470. Agrawal A, Hall NC, Ringel MD, Povoski SP, Martin EW Jr: Combined use of perioperative TSH-stimulated I8F-FDG PET/ CT imaging and gamma probe radioguided surgery to localize and verify resection of iodine scan-negative recurrent thyroid carcinoma. Laryngoscope 2008, I I 8:2190-4.

47I. Helal BO, Merlet P, Tourbert ME, Franc B, Schwartz C, GauthierKoelesnikov H, Prigent A, Syrota A: Clinical impact of (1 8)F-FDG PET in thyroid carcinoma patients with elevated thyroglobulin levels and negative (I3I)I scanning results after therapy. J Nucl Med 200I, 42: I 464-I 469.

472. Grünwald $F$, Kälicke $T$, Feine $U$, Lietzenmayer $R$, Scheidhauer $K$, Dietlein M, Schober O, Lerch H, Brandt-Mainz K, Burchert W, Hiltermann G, Cremerius U, Biersack H: Fluorine- 18 fluorodeoxyglucose positron emission tomography in thyroid cancer: results of a multicentre study. Eur \& NuCl Med 1999, 26:1547-1552.

473. Shaha AR: Management of the neck in thyroid cancer. Otolaryngol Clin North Am 1998, 31:823-831. 
474. Grebe SK, Hay ID: Thyroid cancer nodal metastases: biologic significance and therapeutic considerations. Surg Oncol Clin N Am 1996, 5:43-63.

475. National Comprehensive Cancer Network (NCCN) Clinica Guidelines in Oncology for Thyroid Carcinoma (V.I.2008) [http://www.ncen.org/professionals/physician gls/PDF/thyroid.pdf]

476. Rettenbacher L, Sungler P, Gmeiner D, Kassmann H, Galvan G Detecting the sentinel lymph node in patients with differentiated thyroid carcinoma. Eur J Nucl Med 2000, 27:I399-|40|

477. Catarci M, Zaraca F, Angeloni R, Mancini B, de Filippo MG, Massa R Carboni M, Pasquini G: Preoperative lymphoscintigraphy and sentinel lymph node biopsy in papillary thyroid cancer. A pilot study. J Surg Oncol 200I, 77:2I-24. discussion 25

478. Wiseman S, Hicks W, Chu QD, Rigual NR: Sentinel lymph node biopsy in staging of differential thyroid cancer: a critica review. Surg Oncol 2002, I I: | 37- | 42.

479. Stoeckli SJ, Pfaltz M, Steinert H, Schmid S: Sentinel lymph node biopsy in thyroid tumors: a pilot study. Eur Arch Otorhinolaryngol 2003, 260:364-368.

480. Rubello D, Pelizzo MR, Al-Nahhas A, Salvatori M, O'Doherty MJ, Giuliano AE, Gross MD, Fanti S, Sandrucci S, Casara D, Mariani G: The role of sentinel lymph node biopsy in patients with differentiated thyroid carcinoma. Eur I Surg Oncol 2006 , 32:917-921.

48I. Pelizzo MR, Merante Boschin I, Toniato A, Piotto A, Bernante P, Paggetta C, De Salvo GL, Carpi A, Rubello D, Casara D: Sentinel node mapping and biopsy in thyroid cancer: a surgical perspective. Biomed Pharmacother 2006, 60:405-408.

482. Pelizzo MR, Rubello D, Boschin IM, Piotto A, Paggetta C, Toniato A, De Salvo GL, Giuliano A, Mariani G, Casara D: Contribution of SLN investigation with $99 \mathrm{mTc}$-nanocolloid in clinical staging of thyroid cancer: technical feasibility. Eur J Nucl Med Mol Imaging 2007, 34:934-938.

483. Gould EA, Winship T, Philbin $\mathrm{PH}$, Kerr $\mathrm{HH}$ : Observations on a "sentinel node" in cancer of the parotid. Cancer 1960, I3:77-78.

484. Stárek I, Koranda P, Zboøil V, Mrzena L: Sentinel lymph node biopsy in parotid gland carcinoma. Clin Nucl Med 2006 , 31:203-204

485. Decesare SL, Fiorica JV, Roberts WS, Reintgen D, Arango H, Hoffman MS, Puleo C, Cavanagh D: A pilot study utilizing intraoperative lymphoscintigraphy for identification of the sentinel lymph nodes in vulvar cancer. Gynecol Oncol 1997, 66:425-428.

486. de Hullu JA, Doting E, Piers DA, Hollema H, Aalders JG, Koops HS, Boonstra $H$, Zee AG van der: Sentinel lymph node identification with technetium-99m-labeled nanocolloid in squamous cell cancer of the vulva. J Nucl Med I998, 39:| 38|-|385.

487. Terada KY, Coel MN, Ko P, Wong JH: Combined use of intraoperative lymphatic mapping and lymphoscintigraphy in the management of squamous cell cancer of the vulva. Gynecol Oncol 1998, 70:65-69.

488. Bowles J, Terada KY, Coel MN, Wong JH: Preoperative lymphoscintigraphy in the evaluation of squamous cell cancer of the vulva. Clin Nucl Med 1999, 24:235-238.

489. Rodier JF, Janser JC, Routiot T, David E, Ott G, Schneegans O, Ghnassia JP: Sentinel node biopsy in vulvar malignancies: a preliminary feasibility study. Oncol Rep 1999, 6:1249-1252.

490. Sideri M, De Cicco C, Maggioni A, Colombo N, Bocciolone L, Trifirò G, De Nuzzo M, Mangioni C, Paganelli G: Detection of sentinel nodes by lymphoscintigraphy and gamma probe guided surgery in vulvar neoplasia. Tumori 2000, 86:359-363.

491. De Cicco C, Sideri M, Bartolomei M, Grana C, Cremonesi M, Fiorenza M, Maggioni A, Bocciolone L, Mangioni C, Colombo N, Paganelli G: Sentinel node biopsy in early vulvar cancer. $\mathrm{Br}$ J Cancer 2000, 82:295-299.

492. Molpus KL, Kelley MC, Johnson JE, Martin WH, Jones HW 3rd: Sentinel lymph node detection and microstaging in vulvar carcinoma. J Reprod Med 200I, 46:863-869.

493. Sliutz G, Reinthaller A, Lantzsch T, Mende T, Sinzinger H, Kainz C, Koelbl $\mathrm{H}$ : Lymphatic mapping of sentinel nodes in early vulvar cancer. Gynecol Oncol 2002, 84:449-452.

494. Zámbó K, Schmidt E, Hartmann T, Kornya L, Dehghani B, Tinneberg $H R$, Bódis J: Preliminary experiences with sentinel lymph node detection in cases of vulvar malignancy. Eur I Nucl Med Mol Imaging 2002, 29: 1 198-1200.

495. Puig-Tintoré LM, Ordi J, Vidal-Sicart S, Lejárcegui JA, Torné A, Pahisa J, Iglesias X: Further data on the usefulness of sentinel lymph node identification and ultrastaging in vulvar squamous cell carcinoma. Gynecol Oncol 2003, 88:29-34.

496. Moore RG, Granai CO, Gajewski W, Gordinier M, Steinhoff MM: Pathologic evaluation of inguinal sentinel lymph nodes in vulvar cancer patients: a comparison of immunohistochemical staining versus ultrastaging with hematoxylin and eosin staining. Gynecol Oncol 2003, 91 :378-382.

497. Merisio C, Berretta R, Gualdi M, Pultrone DC, Anfuso S, Agnese G, Aprile C, Mereu L, Salamano S, Tateo S, Melpignano M: Radioguided sentinel lymph node detection in vulvar cancer. Int J Gynecol Cancer 2005, I 5:493-497.

498. Wydra D, Sawicki S, Emerich J, Romanowicz G: Evaluation of sentinel node detection in vulvar cancer. Nucl Med Rev Cent East Eur 2005, 8: $128-130$.

499. Dhar KK, Woolas RP: Lymphatic mapping and sentinel node biopsy in early vulvar cancer. BJOG 2005, I I 2:696-702.

500. Terada KY, Shimizu DM, Jiang CS, Wong JH: Outcomes for patients with TI squamous cell cancer of the vulva undergoing sentinel node biopsy. Gynecol Oncol 2006, I02:200-203.

50I. Nyberg RH, livonen M, Parkkinen J, Kuoppala T, Mäenpää JU: Sentinel node and vulvar cancer: a series of 47 patients. Acta Obstet Gynecol Scand 2007, 86:615-619.

502. Vidal-Sicart S, Puig-Tintoré LM, Lejárcegui JA, Paredes $P$, Ortega $M L$ Muñoz A, Ordi J, Fusté P, Ortín J, Duch J, Martín F, Pons F: Validation and application of the sentinel lymph node concept in malignant vulvar tumours. Eur J Nucl Med Mol Imaging 2007, 34:384-391.

503. Hauspy J, Beiner M, Harley I, Ehrlich L, Rasty G, Coverns A: Sentinel lymph node in vulvar cancer. Cancer 2007, I I 0:1015-1022

504. Rob L, Robova H, Pluta M, Strnad P, Kacirek J, Skapa P, Taborska K: Further data on sentinel lymph node mapping in vulvar cancer by blue dye and radiocolloid Tc99. Int J Gynecol Cancer 2007, I 7:147-153

505. Loar PV 3rd, Reynolds RK: Sentinel lymph node mapping in gynecologic malignancies. Int J Gynaecol Obstet 2007, 99:69-74.

506. Ayhan A, Celik H, Dursun P: Lymphatic mapping and sentinel node biopsy in gynecological cancers: a critical review of the literature. World J Surg Oncol 2008, 6:53

507. Frumovitz M, Levenback CF: Lymphatic mapping and sentinel node biopsy in vulvar, vaginal, and cervical cancers. Oncology (Williston Park) 2008, 22:529-536. discussion 538-539, 542-543

508. Johann S, Klaeser B, Krause T, Mueller MD: Comparison of outcome and recurrence-free survival after sentinel lymph node biopsy and lymphadenectomy in vulvar cancer. Gynecol Oncol 2008, I I 0:324-328.

509. Hampl M, Hantschmann P, Michels W, Hillemanns P: German multicenter study group: validation of the accuracy of the sentinel lymph node procedure in patients with vulvar cancer: results of a multicenter study in Germany. Gynecol Oncol 2008, | | | : 282-288.

510. Levenback C, Tian C, Coleman R, Gold M, Fowler J, Judson P: Sentinel node (SN) biopsy in patients with vulvar cancer, the Gynecologic Oncology Group (GOG) experience. Ann Surg Oncol 2008, I 5(SI):28.

5I I. van Dam P, Sonnemans H, van Dam PJ, Verkinderen L, Dirix LY: Sentinel node detection in patients with vaginal carcinoma. Gynecol Oncol 2004, 92:89-92.

512. Frumovitz M, Gayed IW, Jhingran A, Euscher ED, Coleman RL, Ramirez PT, Levenback CF: Lymphatic mapping and sentinel lymph node detection in women with vaginal cancer. Gynecol Oncol 2008, I 08:478-48I.

5I3. Verheijen RH, Pijpers R, van Diest PJ, Burger CW, Buist MR, Kenemans P: Sentinel node detection in cervical cancer. Obstet Gynecol 2000, 96:135-138.

5I4. Kamprath S, Possover M, Schneider A: Laparoscopic sentinel lymph node detection in patients with cervical cancer. $\mathrm{Am}$ Obstet Gynecol 2000, 182:1648.

5I5. Malur S, Krause N, Köhler C, Schneider A: Sentinel lymph node detection in patients with cervical cancer. Gynecol Oncol 200I, 80:254-257.

516. Lantzsch T, Wolters M, Grimm J, Mende T, Buchmann J, Sliutz G, Koelbl H: Sentinel node procedure in Ib cervical cancer: a preliminary series. Br J Cancer 200I, 85:79I-794.

517. Levenback C, Coleman RL, Burke TW, Lin WM, Erdman W, Deavers $M$, Delpassand ES: Lymphatic mapping and sentinel node identification in patients with cervix cancer undergoing radical 
hysterectomy and pelvic lymphadenectomy. J Clin Oncol 2002, 20:688-693.

518. Rhim CC, Park JS, Bae SN, Namkoong SE: Sentinel node biopsy as an indicator for pelvic nodes dissection in early stage cervical cancer. J Korean Med Sci 2002, I 7:507-5I I.

519. van Dam PA, Hauspy J, Vanderheyden T, Sonnemans H, Spaepen A Eggenstein G, Dirix L, Verkinderen L: Intraoperative sentine node identification with technetium- $99 \mathrm{~m}$-labeled nanocolloid in patients with cancer of the uterine cervix: a feasibility study. Int J Gynecol Cancer 2003, 13: | 82-186.

520. Lambaudie E, Collinet P, Narducci F, Sonoda Y, Papageorgiou T, Carpentier P, Leblanc E, Querleu D: Laparoscopic identification of sentinel lymph nodes in early stage cervical cancer: prospective study using a combination of patent blue dye injection and technetium radiocolloid injection. Gynecol Oncol 2003, 89:84-87.

52I. Chung YA, Kim SH, Sohn HS, Chung SK, Rhim CC, Namkoong SE: Usefulness of lymphoscintigraphy and intraoperative gamma probe detection in the identification of sentinel nodes in cervical cancer. Eur J Nucl Med Mol Imaging 2003, 30:1014-1017

522. Barranger E, Grahek D, Cortez A, Talbot JN, Uzan S, Darai E: Laparoscopic sentinel lymph node procedure using a combination of patent blue and radioisotope in women with cervical carcinoma. Cancer 2003, 97:3003-3009.

523. Buist MR, Pijpers RJ, van Lingen A, van Diest PJ, Dijkstra J, Kenemans $\mathrm{P}$, Verheijen RH: Laparoscopic detection of sentinel lymph nodes followed by lymph node dissection in patients with early stage cervical cancer. Gynecol Oncol 2003, 90:290-296.

524. Pityñski K, Basta A, Oplawski M, Przeszlakowski D, Hubalewska-Hoła A, Krysztopowicz W: [Lymph node mapping and sentinel node detection in carcinoma of the cervix, endometrium and vulva]. Ginekol Pol 2003, 74:830-835. [Polish]

525. Plante M, Renaud MC, Têtu B, Harel F, Roy M: Laparoscopic sentinel node mapping in early-stage cervical cancer. Gynecol Oncol 2003, $91: 494-503$

526. Hubalewska A, Sowa-Staszczak A, Huszno B, Markocka A, Pityñski K, Basta A, Oplawski M, Basta P: Use of Tc99m-nanocolloid for sentinel nodes identification in cervical cancer. Nucl Med Rev Cent East Eur 2003, 6: 127-130.

527. Martínez-Palones JM, Gil-Moreno A, Pérez-Benavente MA, Roca I, Xercavins J: Intraoperative sentinel node identification in early stage cervical cancer using a combination of radiolabeled albumin injection and isosulfan blue dye injection. Gynecol Oncol 2004, 92:845-850.

528. Li B, Zhang WH, Liu L, Wu LY, Zhang R, Li N: Sentinel lymph node identification in patients with early stage cervical cancer undergoing radical hysterectomy and pelvic lymphadenectomy. Chin Med J (Engl) 2004, I I 7:867-870.

529. Wang HY, Sun JM, Tang J: [Sentinel lymph nodes detection in patients with cervical cancer undergoing radical hysterectomy]. Zhonghua Fu Chan Ke Za Zhi 2004, 39:7-9. [Chinese]

530. Sheng XG, Li DP, Liu NF, Li QS, Tang L, Li HQ, Lu CH: [Clinical significance of sentinel lymph nodes detection in patients with early stage cervical cancer]. Zhonghua Fu Chan Ke Za Zhi 2004, 39:10-13. [Chinese]

53I. Barranger E, Cortez A, Commo F, Marpeau O, Uzan S, Darai E, Callard P: Histopathological validation of the sentinel node concept in cervical cancer. Ann Oncol 2004, I 5:870-874.

532. Pijpers R, Buist MR, van Lingen A, Dijkstra J, van Diest PJ, Teule GJ, Kenemans $P$, Verheijen RH: The sentinel node in cervical cancer: scintigraphy and laparoscopic gamma probe-guided biopsy. Eur J Nucl Med Mol Imaging 2004, 3 I: : 479 - I 486.

533. Niikura H, Okamura C, Akahira J, Takano T, Ito K, Okamura K, Yaegashi N: Sentinel lymph node detection in early cervical cancer with combination $99 \mathrm{mTc}$ phytate and patent blue. Gynecol Oncol 2004, 94:528-532

534. Paredes P, Vidal-Sicart S, García S, Pahisa J, Torné A, Ordi J, Fuertes $S$, Ortega $M$, Pons $F$ : [Usefulness of the sentinel node in the treatment and staging of initial cervix cancer]. Rev Esp Med Nucl 2004, 23:253-258. [Spanish]

535. Gil-Moreno A, Díaz-Feijoo B, Roca I, Puig O, Pérez-Benavente MA Aguilar I, Martínez-Palones JM, Xercavins J: Total laparoscopic radical hysterectomy with intraoperative sentinel node identification in patients with early invasive cervical cancer. Gynecol Oncol 2005, 96:187-193.
536. Angioli R, Palaia I, Cipriani C, Muzii L, Calcagno M, Gullotta G, Panici PB: Role of sentinel lymph node biopsy procedure in cervical cancer: a critical point of view. Gynecol Oncol 2005, 96:504-509.

537. Lin YS, Tzeng CC, Huang KF, Kang CY, Chia CC, Hsieh JF: Sentinel node detection with radiocolloid lymphatic mapping in early invasive cervical cancer. Int / Gynecol Cancer 2005, 15:273-277.

538. Silva LB, Silva-Filho AL, Traiman P, Triginelli SA, de Lima CF, Siqueira CF, Barroso A, Rossi TM, Pedrosa MS, Miranda D, Melo JR: Sentinel node detection in cervical cancer with $(99 \mathrm{~m}) \mathrm{Tc}$-phytate. Gynecol Oncol 2005, 97:588-595.

539. Roca I, Caresia AP, Gil-Moreno A, Pifarre P, Aguade-Bruix S, CastellConesa J, Martínez-Palones JM, Xercavins J: Usefulness of sentinel lymph node detection in early stages of cervical cancer. Eur J Nucl Med Mol Imaging 2005, 32:1210-1216.

540. Rob L, Strnad P, Robova H, Charvat M, Pluta M, Schlegerova D, Hrehorcak M: Study of lymphatic mapping and sentinel node identification in early stage cervical cancer. Gynecol Oncol 2005, 98:28I-288

54I. Wydra D, Sawicki S, Wojtylak S, Bandurski T, Emerich J: Sentinel node identification in cervical cancer patients undergoing transperitoneal radical hysterectomy: a study of 100 cases. Int J Gynecol Cancer 2006, 16:649-654.

542. Kraft O, Sevcík L, Klát J, Koliba P, Curík R, Kríozvá H: Detection of sentinel lymph nodes in cervical cancer. A comparison of two protocols. Nucl Med Rev Cent East Eur 2006, 9:65-68.

543. Bats AS, Clément $D$, Larousserie $F$, Lefrère-Belda MA, Faraggi M, Froissart M, Lécuru F: Sentinel lymph node biopsy improves staging in early cervical cancer. Gynecol Oncol 2007, 105:189-193.

544. Hauspy J, Beiner M, Harley I, Ehrlich L, Rasty G, Covens A: Sentinel lymph nodes in early stage cervical cancer. Gynecol Oncol 2007, 105:285-290.

545. Kushner DM, Connor JP, Wilson MA, Hafez GR, Chappell RJ, Stewart SL, Hartenbach EM: Laparoscopic sentinel lymph node mapping for cervix cancer - a detailed evaluation and time analysis. Gynecol Oncol 2007, 1 06:507-5 I2.

546. Lavoué V, Bats AS, Rouzier R, Coutant C, Barranger E, Daraï E: Sentinel lymph node procedure followed by laparoscopic pelvic and paraaortic lymphadenectomy in women with IB2-II cervical cancer. Ann Surg Oncol 2007, | 4:2654-266I.

547. Sevcík L, Klát J, Gráf $P$, Koliba P, Curík R, Kraft O: [Sentinel node detection in early stage of cervical carcinoma using $99 \mathrm{mTc}$. nanocolloid and blue dye]. Ceska Gynekol 2007, 72:120-125. [Czech]

548. Lou HM, Lï WG, Xie X, Yang ZY, Ying Y, Xiao BW, Fang XH: [Sentinel lymph node detection and intra-operational diagnosis in patients with early stage cervical cancer]. Zhonghua Yi Xue Za Zhi 2007, 87:3353-3356. [Chinese]

549. Altgassen C, Hertel H, Brandstädt A, Köhler C, Dürst M, Schneider A, AGO Study Group: Multicenter validation study of the sentinel lymph node concept in cervical cancer: AGO Study Group. Clin Oncol 2008, 26:2943-295I.

550. Fader AN, Edwards RP, Cost M, Kanbour-Shakir A, Kelley JL, Schwartz B, Sukumvanich P, Comerci J, Sumkin J, Elishaev E, Rohan LC: Sentinel lymph node biopsy in early-stage cervical cancer: utility of intraoperative versus postoperative assessment. Gynecol Oncol 2008, I I I:13-17.

55I. Levenback CF: Status of sentinel lymph nodes in cervical cancer. Gynecol Oncol 2007, 107(SuppI I):SI8-SI9.

552. Frumovitz M, Ramirez PT, Levenback CF: Lymphatic mapping and sentinel lymph node detection in women with cervical cancer. Gynecol Oncol 2008, I I 0(Suppl 2):SI7-S20.

553. Lande J van de, Torrenga B, Raijmakers PG, Hoekstra OS, van Baal MW, Brölmann HA, Verheijen RH: Sentinel lymph node detection in early stage uterine cervix carcinoma: a systematic review. Gynecol Oncol 2007, 106:604-613

554. Selman TJ, Mann C, Zamora J, Appleyard TL, Khan K: Diagnostic accuracy of tests for lymph node status in primary cervical cancer: a systematic review and meta-analysis. CMAJ 2008 , 178:855-862.

555. Pelosi E, Arena V, Baudino B, Bellò M, Gargiulo T, Giusti M, Bottero A, Leo L, Armellino F, Palladin D, Bisi G: Preliminary study of sentinel node identification with $99 \mathrm{mTc}$ colloid and blue dye in patients with endometrial cancer. Tumori 2002, 88:S9-S10.

556. Pelosi E, Arena V, Baudino B, Bellò M, Giusti M, Gargiulo T, Palladin $D$, Bisi G: Pre-operative lymphatic mapping and intra-opera- 
tive sentinel lymph node detection in early stage endometrial cancer. Nucl Med Commun 2003, 24:97I-975.

557. Gargiulo T, Giusti M, Bottero A, Leo L, Brokaj L, Armellino F, Palladin L: Sentinel lymph node (SLN) laparoscopic assessment early stage in endometrial cancer. Minerva Ginecol 2003, 55:259-262.

558. Fersis N, Gruber I, Relakis K, Friedrich M, Becker S, Wallwiener D, Wagner U: Sentinel node identification and intraoperative lymphatic mapping. First results of a pilot study in patients with endometrial cancer. Eur I Gynaecol Oncol 2004, 25:339-342.

559. Niikura $H$, Okamura $C$, Utsunomiya $H$, Yoshinaga $K$, Akahira J, Ito $K$, Yaegashi N: Sentinel lymph node detection in patients with endometrial cancer. Gynecol Oncol 2004, 92:669-674.

560. Barranger E, Cortez A, Grahek D, Callard P, Uzan S, Darai E: Laparoscopic sentinel node procedure using a combination of patent blue and radiocolloid in women with endometrial cancer. Ann Surg Oncol 2004, I I:344-349.

561. Raspagliesi F, Ditto A, Kusamura S, Fontanelli R, Vecchione F, Maccauro M, Solima E: Hysteroscopic injection of tracers in sentinel node detection of endometrial cancer: a feasibility study. Am J Obstet Gynecol 2004, I 9 I:435-439.

562. Lelièvre L, Camatte $S$, Le Frère-Belda MA, Kerrou K, Froissart $M$ Taurelle R, Vildé $F$, Lécuru $F$ : [Sentinel lymph node biopsy in cervical and endometrial cancers: a feasibility study]. Bull Cancer 2004, 91 :379-384. [French]

563. Maccauro M, Lucignani G, Aliberti G, Villano C, Castellani MR, Solima $E$, Bombardieri E: Sentinel lymph node detection following the hysteroscopic peritumoural injection of $99 \mathrm{mTc}$-labelled albumin nanocolloid in endometrial cancer. Eur J Nucl Med Mol Imaging 2005, 32:569-574.

564. Bats AS, Clément $D$, Larousserie $F$, Lefrère-Belda MA, Faraggi $M$, Froissart $M$, Lécuru $F$ : [ls sentinel node biopsy feasible in endometrial cancer? Results in 26 patients]. J Gynecol Obstet Biol Reprod (Paris) 2005, 34:768-774. [French]

565. Dzvincuk P, Pilka R, Kudela M, Koranda P: [Sentinel lymph node detection using $99 \mathrm{mTc}$-nanocolloid in endometrial cancer]. Ceska Gynekol 2006, 71:231-236. [Czech]

566. Frumovitz M, Bodurka DC, Broaddus RR, Coleman RL, Sood AK, Gershenson DM, Burke TW, Levenback CF: Lymphatic mapping and sentinel node biopsy in women with high-risk endometrial cancer. Gynecol Oncol 2007, 104:100-103.

567. Delaloye JF, Pampallona S, Chardonnens E, Fiche M, Lehr HA, De Grandi $P$, Delaloye $A B$ : Intraoperative lymphatic mapping and sentinel node biopsy using hysteroscopy in patients with endometrial cancer. Gynecol Oncol 2007, 106:89-93.

568. Niikura H, Okamoto S, Yoshinaga K, Nagase S, Takano T, Ito K, Yaegashi N: Detection of micrometastases in the sentinel lymph nodes of patients with endometrial cancer. Gynecol Oncol 2007 105:683-686.

569. Delpech Y, Cortez A, Coutant C, Callard P, Uzan S, Darai E, Barranger $E$ : The sentinel node concept in endometrial cancer: histopathologic validation by serial section and immunohistochemistry. Ann Oncol 2007, 18:1799-1803.

570. Bats AS, Clément $D$, Larousserie $F$, Le Frère-Belda MA, PierquetGhazzar N, Hignette C, Lécuru F: Does sentinel node biopsy improve the management of endometrial cancer? Data from 43 patients. J Surg Oncol 2008, 97:|4|-|45.

57I. Ballester M, Dubernard G, Rouzier R, Barranger E, Darai E: Use of the sentinel node procedure to stage endometrial cancer. Ann Surg Oncol 2008, 15:1523-1529.

572. Perrone AM, Casadio P, Formelli G, Levorato M, Ghi T, Costa S, Meriggiola MC, Pelusi G: Cervical and hysteroscopic injection for identification of sentinel lymph node in endometrial cancer. Gynecol Oncol 2008, I I I:62-67.

573. Clement D, Bats AS, Ghazzar-Pierquet N, Le Frere Belda MA, Larousserie F, Nos C, Lecuru F: Sentinel lymph nodes in endometrial cancer: is hysteroscopic injection valid? Eur J Gynaecol Oncol 2008, 29:239-24I

574. Khoury-Collado F, Abu-Rustum NR: Lymphatic mapping in endometrial cancer: a literature review of current techniques and results. Int I Gynecol Cancer 2008 in press.

575. Gitsch E, Pateisky N, Schatten C: Diagnostic and intraoperative immunoscintigraphy in ovarian cancer. Eur J Obstet Gynecol Reprod Biol 1989, 32:33-37.

576. Gitsch E, Pateisky N: Radio-immunoscintigraphy and intraoperative tumour detection by means of anti-tumour antibod- ies in patients with ovarian cancer. Baillieres Clin Obstet Gynaecol 1989, 3:3I-36.

577. Pateisky N, Gitsch E: [Immunoscintigraphy and intraoperative tumor search in ovarian cancer]. Arch Gynecol Obstet 1989. 245:606-609. [German]

578. Bell J, Mojzisik C, Hinkle G Jr, Derman H, Schlom J, Martin E: Intraoperative radioimmunodetection of ovarian cancer using monoclonal antibody B72.3 and a portable gamma-detecting probe. Obstet Gynecol 1990, 76:607-6I I.

579. Jäger W, Feistel $H$, Paterok EM, Ronay $G$, Tulusan $A H$, Wolf $F$, Lang $\mathrm{N}$ : Resection guided by antibodies (REGAJ): a diagnostic procedure during second-look operation in ovarian cancer patients. Br J Cancer Suppl 1990, 10:18-20.

580. Krag DN, Haseman MK, Ford P, Smith L, Taylor MH, Schneider $P$, Goodnight JE: Gamma probe location of I I lindium-labeled B72.3: an extension of immunoscintigraphy. J Surg Oncol 1992, $51: 226-230$.

58I. Ind TE, Granowska M, Britton KE, Morris G, Lowe DG, Hudson CN Shepherd JH: Peroperative radioimmunodetection of ovarian carcinoma using a hand-held gamma detection probe. $\mathrm{Br} J$ Cancer 1994, 70: 1263-1266.

582. McIntosh DG, Colcher D, Seemayer T, Smith ML: The intraoperative detection of ovarian adenocarcinoma using radiolabeled CC49 monoclonal antibody and a hand-held gamma-detecting probe. Cancer Biother Radiopharm 1997, 1 2:287-294.

583. Barranger E, Kerrou K, Petegnief Y, David-Montefiore E, Cortez A Daraï E: Laparoscopic resection of occult metastasis using the combination of FDG-positron emission tomography/computed tomography image fusion with intraoperative probe guidance in a woman with recurrent ovarian cancer. Gynecol Oncol 2005, 96:24|-244.

584. Ahmed HU, Arya M, Minhas S: Dynamic sentinel lymph node biopsy in penile cancer. Expert Rev Anticancer Ther 2006, 6:963-967.

585. Cabanas RM: An approach for the treatment of penile carcinoma. Cancer 1977, 39:456-466.

586. Riveros M, Garcia R, Cabañas R: Lymphadenography of the dorsal lymphatics of the penis. Technique and results. Cancer 1967, 20:2026-2031.

587. Perinetti E, Crane DB, Catalona WJ: Unreliability of sentinel lymph node biopsy for staging penile carcinoma. J Urol 1980 , 124:734-735.

588. Fowler JE Jr: Sentinel lymph node biopsy for staging penile cancer. Urology 1984, 23:352-353.

589. Wespes E, Simon J, Schulman CC: Cabanas approach: is sentine node biopsy reliable for staging penile carcinoma? Urology 1986, 28:278-279.

590. Fosså SD, Hall KS, Johannessen NB, Urnes T, Kaalhus O: Cancer of the penis. Experience at the Norwegian Radium Hospital 1 974-1 985. Eur Urol 1987, 1 3:372-377.

59I. Pettaway CA, Pisters LL, Dinney CP, Jularbal F, Swanson DA, von Eschenbach AC, Ayala A: Sentinel lymph node dissection for penile carcinoma: the M. D. Anderson Cancer Center experience. J Urol 1995, 154:1999-2003.

592. Wawroschek F, Vogt H, Bachter D, Weckermann D, Hamm M, Harzmann R: First experience with gamma probe guided sentinel lymph node surgery in penile cancer. Urol Res 2000, 28:246-249.

593. Horenblas S, Jansen L, Meinhardt W, Hoefnagel CA, de Jong D, Nieweg OE: Detection of occult metastasis in squamous cell carcinoma of the penis using a dynamic sentinel node procedure. J Urol 2000, 163:100-104.

594. Valdés Olmos RA, Tanis PJ, Hoefnagel CA, Jansen L, Nieweg OE, Meinhardt W, Horenblas S: Penile lymphoscintigraphy for sentinel node identification. Eur J Nucl Med 200I, 28:58I-585.

595. Akduman B, Fleshner NE, Ehrlich L, Klotz L: Early experience in intermediate-risk penile cancer with sentinel node identification using the gamma probe. Urology 200I, 58:65-68.

596. Benejam Gual JM, García-Miralles Grávalos R, Hidalgo Pardo F, Muñoz Vélez D, Hellín Hellín D, Lago Rodríguez ]: [Usefulness of the dynamic sentinel node technique in patients with penile carcinoma]. Actas Urol Esp 200I, 25:409-4I4. [Spanish]

597. Tanis PJ, Lont AP, Meinhardt W, Olmos RA, Nieweg OE, Horenblas $S$ : Dynamic sentinel node biopsy for penile cancer: reliability of a staging technique. J Urol 2002, 168:76-80. 
598. Lont AP, Horenblas S, Tanis PJ, Gallee MP, van Tinteren H, Nieweg $O E$ : Management of clinically node negative penile carcinoma: improved survival after the introduction of dynamic sentinel node biopsy. J Urol 2003, I 70:783-786.

599. Kroon BK, Horenblas S, Meinhardt W, Poel HG van der, Bex A, van Tinteren $H$, Valdés Olmos RA, Nieweg OE: Dynamic sentinel node biopsy in penile carcinoma: evaluation of 10 years experience. Eur Urol 2005, 47:601-606. discussion 606

600. Perdonà S, Autorino R, De Sio M, Di Lorenzo G, Gallo L, Damiano R, D'Armiento M, Gallo A: Dynamic sentinel node biopsy in clinically node-negative penile cancer versus radical inguinal lymphadenectomy: a comparative study. Urology 2005 , 66: $1282-1286$

60I. Brennhovd B, Johnsrud K, Berner A, Bogsrud T, Waehre H, Giercksky KE, Axcrona K: Sentinel node procedure in low-stage/lowgrade penile carcinomas. Scand J Urol Nephrol 2006, 40:204-207.

602. Perdonà S, Autorino R, Gallo L, Di Lorenzo G, Cascini GL, Lastoria F, Marra L, De Sio M, Damiano R, Gallo A: Role of dynamic sentinel node biopsy in penile cancer: our experience. J Surg Oncol 2006, 93: $181-185$.

603. Leijte JA, Kroon BK, Valdés Olmos RA, Nieweg OE, Horenblas S: Reliability and safety of current dynamic sentinel node biopsy for penile carcinoma. Eur Urol 2007, 52:170-177.

604. Gonzaga-Silva LF, Tavares JM, Freitas FC, Tomas Filho ME, Oliveira VP, Lima MV: The isolated gamma probe technique for sentinel node penile carcinoma detection is unreliable. Int Braz J Urol 2007, 33:58-63. discussion 64-67

605. Spiess PE, Izawa JI, Bassett R, Kedar D, Busby JE, Wong F, Eddings T, Tamboli P, Pettaway CA: Preoperative lymphoscintigraphy and dynamic sentinel node biopsy for staging penile cancer: results with pathological correlation. J Urol 2007 |77:2157-2161.

606. Hernández-Toris N, Quintero-Becerra J, Gallegos-Hernández JF, Flores-Ojeda R, Alvarado-Cabrero I, Flores-López D, PichardoRomero P: [Lymphatic mapping and sentinel node biopsy in penis cancer. Feasibility study and preliminary report]. Cir Cir 2007, 75:87-9I. [Spanish]

607. Hadway P, Smith Y, Corbishley C, Heenan S, Watkin NA: Evaluation of dynamic lymphoscintigraphy and sentinel lymph-node biopsy for detecting occult metastases in patients with penile squamous cell carcinoma. BJU Int 2007, I 00:56I-565.

608. Rubí S, Vidal-Sicar S, Ortega M, Doménech B, Lafuente S, Corral JM, Gelabert-Mas A: [Localization of sentinel node in squamous cell carcinoma of the penis. Initial experience]. Rev Esp Med Nucl 2008, 27:3-7. [Spanish]

609. Heyns CF, Theron PD: Evaluation of dynamic sentinel lymph node biopsy in patients with squamous cell carcinoma of the penis and palpable inguinal nodes. BJU Int 2008, I 02:305-309.

610. Wawroschek F, Vogt H, Weckermann D, Wagner T, Harzmann R: The sentinel lymph node concept in prostate cancer - first results of gamma probe-guided sentinel lymph node identification. Eur Urol 1999, 36:595-600.

6II. Wawroschek F, Vogt H, Weckermann D, Wagner T, Hamm M, Harzmann R: Radioisotope guided pelvic lymph node dissection for prostate cancer. JUrol 2001, 166:1715-1719.

612. Wengenmair H, Kopp J, Vogt H, Wawroschek F, Gröber S, Dorn R, Heidenreich $P$ : [Sentinel lymph node diagnosis in prostatic carcinoma: II. Biokinetics and dosimetry of $99 \mathrm{mT}$-nanocolloid after intraprostatic injection]. Nuklearmedizin 2002, 4 I: I 02- 107. [German]

613. Vogt H, Wawroschek F, Wengenmair H, Wagner T, Kopp J, Dorn R Gröber S, Heidenreich P: [Sentinel lymph node diagnosis in prostatic carcinoma: I: Method and clinical evaluation]. Nuklearmedizin 2002, 41:95-I01. [German]

6I4. Rudoni M, Sacchetti GM, Leva L, Inglese E, Monesi G, Minocci D, Frea $B$ : Recent applications of the sentinel lymph node concept: preliminary experience in prostate cancer. Tumori 2002 88:SI6-SI7.

615. Wawroschek F, Vogt H, Wengenmair H, Weckermann D, Hamm M, Keil M, Graf G, Heidenreich P, Harzmann R: Prostate lymphoscintigraphy and radio-guided surgery for sentinel lymph node identification in prostate cancer. Technique and results of the first 350 cases. Urol Int 2003, 70:303-310.

616. Takashima H, Egawa M, Imao T, Fukuda M, Yokoyama K, Namiki M: Validity of sentinel lymph node concept for patients with prostate cancer. J Urol 2004, I 7 I:2268-227I.
617. Egawa M, Fukuda M, Takashima H, Imao T, Namiki M: [Application of sentinel node navigation surgery to prostate cancer]. Gan To Kagaku Ryoho 2005, 32: I I7-120. Japanese

618. Bastide C, Brenot-Rossi I, Garcia S, Dumas S, Anfossi E, Ragni E, Rossi $D$ : [Feasibility and value of the isotope sentinel node mapping technique in prostate cancer]. Prog Urol 2004, 14:50 I-506. [French]

619. Brenot-Rossi I, Bastide C, Garcia S, Dumas S, Esterni B, Pasquier J, Rossi D: Limited pelvic lymphadenectomy using the sentinel lymph node procedure in patients with localised prostate carcinoma: a pilot study. Eur J Nucl Med Mol Imaging 2005, 32:635-640.

620. Jeschke S, Nambirajan T, Leeb K, Ziegerhofer J, Sega W, Janetschek G: Detection of early lymph node metastases in prostate cancer by laparoscopic radioisotope guided sentinel lymph node dissection. J Urol 2005, I 73:1943-1946.

62I. Silva N Jr, Anselmi CE, Anselmi OE, Madke RR, Hunsche A, Souto JS, Souto CA, Sica FD, Pioner GT, Macalos EC, Hartmann AA, Lima MS: Use of the gamma probe in sentinel lymph node biopsy in patients with prostate cancer. Nucl Med Commun 2005, 26: $1081-1086$.

622. Corvin S, Schilling D, Eichhorn K, Hundt I, Hennenlotter J, Anastasiadis AG, Kuczyk M, Bares R, Stenzl A: Laparoscopic sentinel lymph node dissection - a novel technique for the staging of prostate cancer. Eur Urol 2006, 49:280-285.

623. Weckermann D, Hamm M, Dorn R, Wagner T, Wawroschek F, Harzmann R: [Sentinel lymph node dissection in prostate cancer. Experience after more than $\mathbf{8 0 0}$ interventions]. Urologe $A$ 2006, 45:723-727. [German]

624. Häcker A, Jeschke S, Leeb K, Prammer K, Ziegerhofer J, Sega W, Langsteger W, Janetschek G: Detection of pelvic lymph node metastases in patients with clinically localized prostate cancer: comparison of [I8F]fluorocholine positron emission tomography-computerized tomography and laparoscopic radioisotope guided sentinel lymph node dissection. J Urol 2006, I 76:2014-2018. discussion 2018-2019

625. Weckermann D, Dorn R, Holl G, Wagner T, Harzmann R: Limitations of radioguided surgery in high-risk prostate cancer. Eur Urol 2007, 5 I: I549-1556. discussion 1556-I558

626. Warncke SH, Mattei A, Fuechsel FG, Z'Brun S, Krause T, Studer UE: Detection rate and operating time required for gamma probe-guided sentinel lymph node resection after injection of technetium-99m nanocolloid into the prostate with and without preoperative imaging. Eur Urol 2007, 52:I26-I32.

627. Weckermann D, Dorn R, Trefz M, Wagner T, Wawroschek F, Harzmann R: Sentinel lymph node dissection for prostate cancer: experience with more than $\mathbf{1 , 0 0 0}$ patients. J Urol 2007, I 77:916-920.

628. Fukuda M, Egawa M, Imao T, Takashima H, Yokoyama K, Namiki M: Detection of sentinel node micrometastasis by step section and immunohistochemistry in patients with prostate cancer. J Urol 2007, 177:1313-1317. discussion 1317

629. Jeschke S, Beri A, Grüll M, Ziegerhofer J, Prammer P, Leeb K, Sega W Janetschek G: Laparoscopic radioisotope-guided sentinel lymph node dissection in staging of prostate cancer. Eur Urol 2008, 53:126-132.

630. Brenot-Rossi I, Rossi D, Esterni B, Brunelle S, Chuto G, Bastide C: Radioguided sentinel lymph node dissection in patients with localised prostate carcinoma: influence of the dose of radiolabelled colloid to avoid failure of the procedure. Eur J Nucl Med Mol Imaging 2008, 35:32-38.

631. Hautmann S, Beitz S, Naumann M, Lützen U, Seif C, Stübinger SH, Horst C van der, Braun PM, Leuschner I, Henze E, Jünemann KP: [Extended sentinel lymph node dissection in radical prostatectomy for prostate cancer: a study in the Kiel risk population]. Urologe A 2008, 47:299-303. [German]

632. Meinhardt W, Valdés Olmos RA, Poel HG van der, Bex A, Horenblas $S$ : Laparoscopic sentinel node dissection for prostate carcinoma: technical and anatomical observations. BJU Int 2008 , 102:7|4-7|7.

633. Badalament RA, Burgers JK, Petty LR, Mojzisik CM, Berens A, Marsh W, Hinkle GH, Martin EW Jr: Radioimmunoguided radical prostatectomy and lymphadenectomy. Cancer 1993, 71:2268-2275.

634. Anderson RS, Eifert B, Tartt S, King P: Radioimmunoguided surgery using indium-III capromab pendetide (PROS- 
TASCINT) to diagnose supraclavicular metastasis from prostate cancer. Urology 2000, 56:669xii-669xiv.

635. Tanis PJ, Horenblas S, Valdés Olmos RA, Hoefnagel CA, Nieweg OE: Feasibility of sentinel node lymphoscintigraphy in stage $I$ testicular cancer. Eur J Nucl Med Mol Imaging 2002, 29:670-673.

636. Ohyama C, Chiba Y, Yamazaki T, Endoh M, Hoshi S, Arai Y: Lymphatic mapping and gamma probe guided laparoscopic biopsy of sentinel lymph node in patients with clinical stage $I$ testicular tumor. J Urol 2002, I68: | 390-I395.

637. Satoh M, Ito A, Kaiho Y, Nakagawa H, Saito S, Endo M, Ohyama C, Arai $Y$ : Intraoperative, radio-guided sentinel lymph node mapping in laparoscopic lymph node dissection for Stage I testicular carcinoma. Cancer 2005, 103:2067-2072.

638. National Comprehensive Cancer Network (NCCN) Clinical Guidelines in Oncology for Testicular Cancer (V.I.2009) [http://www.ncen.org/professionals/physician gls/PDF/testicular.pdf]

639. Sherif A, De La Torre M, Malmström PU, Thörn M: Lymphatic mapping and detection of sentinel nodes in patients with bladder cancer. J Urol 200I, 166:8I2-8I5.

640. Liedberg F, Chebil G, Davidsson T, Malmström PU, Sherif A, Thörn M, De La Torre M, Månsson W: [Bladder cancer and the sentinel node concept]. Aktuelle Urol 2003, 34: I I5-II8. [German]

64I. Liedberg F, Chebil G, Davidsson T, Gudjonsson S, Månsson W: Intraoperative sentinel node detection improves nodal staging in invasive bladder cancer. J Urol 2006, 175:84-88. discussion 88-89

642. Sherif A, Garske U, de la Torre M, Thörn M: Hybrid SPECT-CT: an additional technique for sentinel node detection of patients with invasive bladder cancer. Eur Urol 2006, 50:83-91.

643. Avital S, Hitchcock CL, Baratz M, Haddad R, Skornick Y, Schneebaum $\mathrm{S}$ : Localization of monoclonal antibody CC49 in colonic metastasis from renal cell carcinoma. Eur J Surg Oncol 1998, 24:|49-|5|.

644. Oosterwijk E, Bander NH, Divgi CR, Welt S, Wakka JC, Finn RD Carswell EA, Larson SM, Warnaar SO, Fleuren G], Oettgen HF, Old LJ: Antibody localization in human renal cell carcinoma: a phase I study of monoclonal antibody G250. J Clin Oncol 1993, II:738-750.

645. Uemura H, Nakagawa Y, Yoshida K, Saga S, Yoshikawa K, Hirao Y, Oosterwijk E: MN/CA IX/G250 as a potential target for immunotherapy of renal cell carcinomas. $\mathrm{Br}$ / Cancer 1999, $81: 74 I-746$.

646. Divgi CR, Pandit-Taskar N, Jungbluth AA, Reuter VE, Gönen M, Ruan S, Pierre C, Nagel A, Pryma DA, Humm J, Larson SM, Old LJ, Russo $\mathrm{P}$ : Preoperative characterisation of clear-cell renal carcinoma using iodine-124-labelled antibody chimeric G250 ( I 24I-cG250) and PET in patients with renal masses: a phase I trial. Lancet Oncol 2007, 8:304-310.

647. Woolfenden JM, Nevin WS, Barber HB, Donahue DJ: Lung cancer detection using a miniature sodium iodide detector and cobalt-57 bleomycin. Chest 1984, 85:84-88.

648. Liptay MJ, Masters GA, Winchester DJ, Edelman BL, Garrido BJ, Hirschtritt TR, Perlman RM, Fry WA: Intraoperative radioisotope sentinel lymph node mapping in non-small cell lung cancer. Ann Thorac Surg 2000, 70:384-389.

649. Nomori H, Horio H, Naruke T, Orikasa H, Yamazaki K, Suemasu K: Use of technetium-99m tin colloid for sentinel lymph node identification in non-small cell lung cancer. J Thorac Cardiovasc Surg 2002, 1 24:486-492.

650. Liptay MJ, Grondin SC, Fry WA, Pozdol C, Carson D, Knop C, Masters GA, Perlman RM, Watkin W: Intraoperative sentinel lymph node mapping in non-small-cell lung cancer improves detection of micrometastases. / Clin Oncol 2002, 20:1984-1988.

65I. Schmidt FE, Woltering EA, Webb WR, Garcia OM, Cohen JE, Rozans $\mathrm{MH}$ : Sentinel nodal assessment in patients with carcinoma of the lung. Ann Thorac Surg 2002, 74:870-874. discussion 874-875

652. Sugi K, Fukuda M, Nakamura H, Kaneda Y: Comparison of three tracers for detecting sentinel lymph nodes in patients with clinical N0 lung cancer. Lung Cancer 2003, 39:37-40.

653. Melfi FM, Chella A, Menconi GF, Givigliano F, Boni G, Mariani G, Sbragia $P$, Angeletti CA: Intraoperative radioguided sentinel lymph node biopsy in non-small cell lung cancer. Eur J Cardiothorac Surg 2003, 23:214-220.

654. Lardinois D, Brack T, Gaspert A, Spahr T, Schneiter D, Steinert HC Weder W: Bronchoscopic radioisotope injection for sentinel lymph-node mapping in potentially resectable non-small-cell lung cancer. Eur J Cardiothorac Surg 2003, 23:824-827.
655. Sugi K, Kaneda Y, Sudoh M, Sakano H, Hamano K: Effect of radioisotope sentinel node mapping in patients with CTI NO MO lung cancer. J Thorac Cardiovasc Surg 2003, I 26:568-573.

656. Sugi K, Kitada K, Murakami T, Matsuda E, Hirazawa K, Azuma T, Umemori Y: [Sentinel node biopsy for staging of small peripheral lung cancer]. Kyobu Geka 2004, 57:14-17. [Japanese]

657. Liptay MJ: Sentinel node mapping in lung cancer. Ann Surg Oncol 2004, I I:27 IS-274S.

658. Ueda K, Suga K, Kaneda Y, Sakano H, Tanaka T, Hayashi M, Li TS, Hamano K: Radioisotope lymph node mapping in nonsmall cell lung cancer: can it be applicable for sentinel node biopsy? Ann Thorac Surg 2004, 77:426-430.

659. Nomori H, Watanabe K, Ohtsuka T, Naruke T, Suemasu K: In vivo identification of sentinel lymph nodes for clinical stage I nonsmall cell lung cancer for abbreviation of mediastinal lymph node dissection. Lung Cancer 2004, 46:49-55.

660. Tiffet O, Nicholson AG, Khaddage A, Prévot N, Ladas G, Dubois F, Goldstraw P: Feasibility of the detection of the sentinel lymph node in peripheral non-small cell lung cancer with radio isotopic and blue dye techniques. Chest 2005, 127:443-448.

66l. Atinkaya C, Ozlem Küçük N, Koparal H, Aras G, Sak SD, Ozdemir N: Mediastinal intraoperative radioisotope sentinel lymph node mapping in non-small-cell lung cancer. Nucl Med Commun 2005 26:717-720.

662. Rzyman W, Hagen OM, Dziadziuszko R, Kobierska-Gulida G, Karmolinski A, Lothe IM, Babovic A, Murawski M, Paleczka W, Jastrzebski T, Kopacz A, Jassem J, Lass P, Skokowski J: Intraoperative, radioguided sentinel lymph node mapping in 110 nonsmall cell lung cancer patients. Ann Thorac Surg 2006, 82:237-242.

663. Nomori H, Ikeda K, Mori T, Kobayashi H, Iwatani K, Kawanaka K, Shiraishi S, Kobayashi T: Sentinel node navigation segmentectomy for clinical stage IA non-small cell lung cancer. J Thorac Cardiovasc Surg 2007, 133:780-785

664. Nomori H, Ikeda K Mori T, Shiraishi S, Kobayashi H, Iwatani K, Kawanaka $K$, Kobayashi T: Sentinel node identification in clinical stage la non-small cell lung cancer by a combined single photon emission computed tomography/computed tomography system. J Thorac Cardiovasc Surg 2007, 134:182-187.

665. Meyer A, Cheng C, Antonescu C, Pezzetta E, Bischof-Delaloye A, Ris $H B$ : Successful migration of three tracers without identification of sentinel nodes during intraoperative lymphatic mapping for non-small cell lung cancer. Interact Cardiovasc Thorac Surg 2007, 6:2।4-218.

666. Di Lieto E, Gallo G, Scarpati VD, Di Tommaso P, Carpenito A, Perrone $A$ : [Lymph node sentinel detection in lung resection for non small cell lung cancer: our experience]. Recenti Prog Med 2007, 98:327-328. [Italian]

667. Melfi FM, Lucchi M, Davini F, Viti A, Fontanini G, Boldrini L, Boni G, Mussi $A$ : Intraoperative sentinel lymph node mapping in stage I non-small cell lung cancer: detection of micrometastases by polymerase chain reaction. Eur J Cardiothorac Surg 2008, 34:181-186.

668. Sugi K, Kobayashi S, Yagi R, Matsuoka T: Usefulness of sentinel lymph node biopsy for the detection of lymph node micrometastasis in early lung cancer. Interact Cardiovasc Thorac Surg 2008, 7:913-915.

669. Liptay MJ: Sentinel node mapping in lung cancer: the Holy Grail? Ann Thorac Surg 2008, 85:S778-S779.

670. Grazia M, Bini A, Stella F, Pagani D, Bazzocchi R: Radioimmunoguided surgery and intraoperative lung cancer staging. Semin Surg Oncol 1998, 15:215-219.

67I. Mansi L, Di Lieto E, Rambaldi PF, Bergaminelli C, Fallanca F, Vicidomini $G$, Cuccurullo V, Mancusi R: [Preliminary experience with radioimmuno-guided surgery of primary neoplasms of the lung]. Minerva Chir 1998, 53:369-372.

672. Wang YQ, Sun YE, Zhang JM, Liu X: [Clinical practice of 99mTcpeplimycin imaging and radio guided surgery for lung neoplasms]. Ai Zheng 2003, 22:749-752.

673. Nwogu C, Fischer G, Tan D, Glinianski M, Lamonica D, Demmy T: Radioguided detection of lymph node metastasis in nonsmall cell lung cancer. Ann Thorac Surg 2006, 82:1815-1820.

674. Moffatt-Bruce SD, Povoski SP, Sharif S, Hall NC, Ross P Jr, Johnson MA, Martin EW Jr: A novel approach to positron emission tomography in lung cancer. Ann Thorac Surg 2008, 86: I355- I357.

675. Chella A, Lucchi M, Ambrogi MC, Menconi G, Melfi FM, Gonfiotti A Boni G, Angeletti CA: A pilot study of the role of TC-99 radio- 
nuclide in localization of pulmonary nodular lesions for thoracoscopic resection. Eur J Cardiothorac Surg 2000, 18:17-2I.

676. Boni G, Bellina CR, Grosso M, Lucchi M, Manca G, Ambrogi MC, Volterrani D, Menconi G, Melfi FM, Gonfiotti A, Davini F, Angeletti CA, Bianchi R, Chella A: Gamma probe-guided thoracoscopic surgery of small pulmonary nodules. Tumori 2000, 86:364-366.

677. Sortini D, Feo CV, Carrella G, Bergossi L, Soliani G, Carcoforo P, Pozza E, Sortini A: Thoracoscopic localization techniques for patients with a single pulmonary nodule and positive oncological anamnesis: a prospective study. J Laparoendosc Adv Surg Tech A 2003, 13:37I-375.

678. Daniel TM, Altes TA, Rehm PK, Williams MB, Jones DR, Stolin AV, Gay SB: A novel technique for localization and excisional biopsy of small or ill-defined pulmonary lesions. Ann Thorac Surg 2004, 77: 1756-1762. discussion 1762

679. Sortini D, Feo CV, Carcoforo P, Carrella G, Pozza E, Liboni A, Sortini A: Thoracoscopic localization techniques for patients with solitary pulmonary nodule and history of malignancy. Ann Thorac Surg 2005, 79:258-262. discussion 262

680. Stiles BM, Altes TA, Jones DR, Shen KR, Ailawadi G, Gay SB, Olazagasti J, Rehm PK, Daniel TM: Clinical experience with radiotracer-guided thoracoscopic biopsy of small, indeterminate lung nodules. Ann Thorac Surg 2006, 82:1191-1196. discussion II96-1197

68I. Davini F, Gonfiotti A, Vaggelli L, De Francisci A, Gigli P, Janni A: Thoracoscopic localization techniques for patients with solitary pulmonary nodule: radioguided surgery versus hookwire localization. J Cardiovasc Surg (Torino) 2006, 47:355-359.

682. Gonfiotti A, Davini F, Vaggelli L, De Francisci A, Caldarella A, Gigli PM, Janni A: Thoracoscopic localization techniques for patients with solitary pulmonary nodule: hookwire versus radio-guided surgery. Eur J Cardiothorac Surg 2007, 32:843-847.

683. Grogan EL, Jones DR, Kozower BD, Simmons WD, Daniel TM: Identification of small lung nodules: technique of radiotracerguided thoracoscopic biopsy. Ann Thorac Surg 2008, 85:S772-S777.

684. Adams S, Baum RP: Intraoperative use of gamma-detecting probes to localize neuroendocrine tumors. QJ Nucl Med 2000, 44:59-67.

685. Gulec SA, Mountcastle TS, Frey D, Cundiff JD, Mathews E, Anthony L, O'Leary JP, Boudreaux JP: Cytoreductive surgery in patients with advanced-stage carcinoid tumors. Am Surg 2002. 68:667-67|. discussion 67|-672

686. Åkerström G, Hellman P, Hessman O, Osmak L: Management of midgut carcinoids. I Surg Oncol 2005, 89:16I-169.

687. Gulec SA, Baum R: Radioguided Surgery in Neuroendocrine Tumors. J Surg Oncol 2007, 96:309-315.

688. Krenning EP, Kwekkeboom DJ, Bakker WH, Breeman WA, Kooij PP, Oei HY, van Hagen M, Postema PT, de Jong M, Reubi JC, Visser TJ, Reijs AEM, Hofland LJ, Koper JW, Lamberts SWJ: Somatostatin receptor scintigraphy with [I I I In-DTPA-D-Phe I]- and [I 23 Tyr3]-octreotide: the Rotterdam experience with more than 1000 patients. Eur J Nucl Med 1993, 20:7|6-73|.

689. Schillaci O, Spanu A, Scopinaro F, Flachi A, Danieli R, Marongiu P, Pisu N, Madeddu G, Delle Fave G, Madeddu G: Somatostatin receptor scintigraphy in liver metastasis detection from gastroenteropancreatic neuroendocrine tumors. I Nucl Med 2003, 44:359-368

690. Yüksel M, Eziddin S, Ladwein E, Haas S, Biersack HJ: I I IIn-pentetreotide and I23I-MIBG for detection and resection of lymph node metastases of a carcinoid not visualized by $C T$, MRI or FDG-PET. Ann Nucl Med 2005, 19:6 I I-6I5.

691. Åkerströom G, Makridis C, Johansson H: Abdominal surgery in patients with midgut carcinoid tumors. Acta Oncol I991, 30:547-553.

692. Norton JA: Intraoperative methods to stage and localize pancreatic and duodenal tumors. Ann Oncol 1999, 10:182-184.

693. Öhrvall U, Westlin J, Nilsson S, Juhlin C, Rastad J, Lundqvist H, Åkerström G: Intraoperative gamma detection reveals abdominal endocrine tumors more efficiently than somatostatin receptor scintigraphy. Cancer 1997, 80:2490-2494.

694. Adams S, Baum R, Hertel A, Wenisch H, Staib-Sebler E, Herrmann G, Encke A, Hör G: Intraoperative Gamma Probe Detection of Neuroendocrine Tumors. J Nucl Med 1998, 39: I I55-I I60.

695. Pelaez N, Busquets J, Ortega M, Martinez Miralles E, Puig F, Miret M, Munne A, Grande L: Intraoperative gamma probe detection of lymph node recurrence of insulinoma. J Surg Oncol 2005, 91:209-2II.

696. Schirmer WJ, O'Dorisio TM, Schirmer TP, Mojzisik CM, Hinkle GH, Martin EW: Intraoperative localization of neuroendocrine tumors with I25I-TYR(3)-octreotide and $a$ hand-held gamma-detecting probe. Surgery 1993, I I 4:745-75 I. discussion 75I-752

697. Benevento A, Dominioni L, Carcano G, Dionigi R: Intraoperative localization of gut endocrine tumors with radiolabeled somatostatin analogs and a gamma-detecting probe. Semin Surg Oncol 1998, 15:239-244.

698. Hubalewska-Dydejczyk A, Kulig J, Szybinski P, Mikolajczak R, Pach D, Sowa-Staszczak A, Fröss-Baron K, Huszno B: Radio-guided surgery with the use of [99mTc-EDDA/HYNIC] octreotate in intra-operative detection of neuroendocrine tumours of the gastrointestinal tract. Eur J Nucl Med Mol Imaging 2007, 34:1545-I555.

699. Touzios JG, Kiely JM, Pitt SC, Rilling WS, Quebbeman EJ, Wilson SD, Pitt HA: Neuroendocrine hepatic metastases: does aggressive management improve survival? Ann Surg 2005, 241:776-783. discussion 783-785

700. Maecke HR, Hofmann M, Haberkorn U: (68)Ga-labeled peptides in tumor imaging. I Nucl Med 2005, 46(Suppl I): I72S-178S

70I. Arbizu J, Rodriguez-Fraile M, Dominguez-Prado I, Garrastachu P, Rotellar F, Sangro B, Richter JA: Whole body I 8fluoro-L-dopa PET-CT: a useful tool for location and surgical guidance in primary carcinoid tumours. Eur J Nucl Med Mol lmaging 2008, 35:1577.

702. Mansi L, Rambaldi PF, Panza N, Esposito D, Esposito V, Pastore V: Diagnosis and radioguided surgery with I I I In-pentetreotide in a patient with paraneoplastic Cushing's syndrome due to a bronchial carcinoid. Eur I Endocrinol 1997, 137:688-690.

703. Grossrubatscher E, Vignati F, Dalino P, Possa M, Belloni PA, Vanzulli A, Bramerio M, Marocchi A, Rossetti O, Zurleni F, Loli P: Use of radioguided surgery with [ $I I I I n]$-pentetreotide in the management of an ACTH-secreting bronchial carcinoid causing ectopic Cushing's syndrome. J Endocrinol Invest 2005, 28:72-78.

704. Martinez DA, O'Dorisio MS, O'Dorisio TM, Qualman SJ, Caniano DA, Teich S, Besner GE, King DR: Intraoperative detection and resection of occult neuroblastoma: a technique exploiting somatostatin receptor expression. I Pediatr Surg 1995, 30:1580-1589.

705. Heij HA, Rutgers EJ, de Kraker J, Vos A: Intraoperative search for neuroblastoma by MIBG and radioguided surgery with the gamma detector. Med Pediatr Oncol 1997, 28:17|-174.

706. Martelli H, Ricard M, Larroquet M, Wioland M, Paraf F, Fabre M, Josset P, Helardot PG, Gauthier F, Terrier-Lacombe MJ, Michon J, Harmann O, Tabone MD, Patte C, Lumbroso J, Gruner M: Intraoperative localization of neuroblastoma in children with |23|- or |25|-radiolabelled metaiodobenzylguanidine. Surgery 1998, I 23:5I-57.

707. lagaru A, Peterson D, Quon A, Dutta S, Twist C, Daghighian F, Gambhir SS, Albanese C: I23I MIBG mapping with intraoperative gamma probe for recurrent neuroblastoma. Mol Imaging Biol 2008, 10:19-23

708. Hoefnagel CA: Metaiodobenzylguanidine and somatostatin in oncology: role in the management of neural crest tumors. Eur J Nucl Med 1994, 21:561-581.

709. Fasshauer H, Freundlieb O, Dostal G, Littmann K, Tharandt L, Strötges MW: [Intraoperative localization of pheochromocytoma metastases using I 3 | |-meta-benzylguanidine]. Nuklearmedizin 1984, 23:203-205. [German]

7I0. Proye CA, Carnaille BM, Flament JB, Hossein-Foucher CA, Lecouffe PP, Marchandise XM, Lennquist S: Intraoperative radionuclear |25|-labeled metaiodobenzylguanidine scanning of pheochromocytomas and metastases. Surgery 1992, I I 1:634-639.

7II. Ricard M, Tenenbaum F, Schlumberger M, Travagli JP, Lumbroso J Revillon Y, Parmentier C: Intraoperative detection of pheochromocytoma with iodine- 125 labelled metaiodobenzylguanidine: a feasibility study. Eur J Nucl Med 1993, 20:426-430.

7I2. Blazer DG 3rd, Sabel MS, Sondak VK: Is there a role for sentinel lymph node biopsy in the management of sarcoma? Surg Oncol 2003, I 2:201-206.

713. Neville HL, Andrassy RJ, Lally KP, Corpron C, Ross MI: Lymphatic mapping with sentinel node biopsy in pediatric patients. Pediatr Surg 2000, 35:961-964. 
714. McMulkin HM, Yanchar NL, Fernandez CV, Giacomantonio C: Sentinel lymph node mapping and biopsy: a potentially valuable tool in the management of childhood extremity rhabdomyosarcoma. Pediatr Surg Int 2003, 19:453-456.

715. Al-Refaie WB, Ali MW, Chu DZ, Paz IB, Blair SL: Clear cell sarcoma in the era of sentinel lymph node mapping. J Surg Oncol 2004, 87: I 26- I29.

716. Picciotto F, Zaccagna A, Derosa G, Pisacane A, Puiatti P, Colombo E, Dardano $F$, Ottinetti $A$ : Clear cell sarcoma (malignant melanoma of soft parts) and sentinel lymph node biopsy. Eur J Dermatol 2005, I5:46-48.

717. Seal A, Tse R, Wehrli B, Hammond A, Temple CL: Sentinel node biopsy as an adjunct to limb salvage surgery for epithelioid sarcoma of the hand. World J Surg Oncol 2005, 3:41.

7l8. van Akkooi AC, Verhoef C, van Geel AN, Kliffen M, Eggermont AM, de Wilt JH: Sentinel node biopsy for clear cell sarcoma. Eur J Surg Oncol 2006, 32:996-999.

719. Albores-Zúñiga $O$, Padilla-Rosciano AE, Martínez-Said H, CuéllarHubbe M, Ramírez-Bollas ]: [Clear cell sarcoma and sentinel lymph node biopsy. Case report and literature review]. Cir Cir 2006, 74: $121-125$. [Spanish]

720. Tunn PU, Andreou D, Illing H, Fleige B, Dresel S, Schlag PM: Sentinel node biopsy in synovial sarcoma. Eur J Surg Oncol 2008, 34:704-707.

72I. Kayton ML, Delgado R, Busam K, Cody HS 3rd, Athanasian EA, Coit D, La Quaglia MP: Experience with 3 I sentinel lymph node biopsies for sarcomas and carcinomas in pediatric patients. Cancer 2008, I I 2:2052-2059.

722. De Corti F, Dall'igna P, Bisogno G, Casara D, Rossi CR, Foletto M, Alaggio R, Carli M, Cecchetto G: Sentinel node biopsy in pediatric soft tissue sarcomas of extremities. Pediatr Blood Cancer 2009, 52:5I-54.

723. Vilela Filho O, Carneiro Filho O: Gamma probe-assisted brain tumor microsurgical resection: a new technique. Arq Neuropsiquiatr 2002, 60: 1042-1047

724. Kojima T, Kumita S, Yamaguchi F, Mizumura S, Kitamura T, Kumazaki $\mathrm{T}$, Teramoto $\mathrm{A}$ : Radio-guided brain tumorectomy using a gamma detecting probe and a mobile solid-state gamma camera. Surg Neurol 2004, 6 I:229-238. discussion 238

725. Gay E, Vuillez JP, Palombi O, Brard PY, Bessou P, Passagia JG: Intraoperative and postoperative gamma detection of somatostatin receptors in bone-invasive en plaque meningiomas. Neurosurgery 2005, 57(I Suppl): | 07- I I2. discussion I |2-I I3

726. Serrano J, Rayo JI, Infante JR, Domínguez ML, Lorenzana L, Porras JL, Cabezudo JM, García-Bernardo L, Sánchez-Sánchez R: [Radioguided neurosurgery: a novel application of nuclear medicine]. Rev Esp Med Nucl 2006, 25: 184-187. [Spanish]

727. Bhanot $Y$, Rao S, Parmeshwaran RV: Radio-guided neurosurgery (RGNS): early experience with its use in brain tumour surgery. Br J Neurosurg 2007, 2 1:382-388.

728. Ghelman B, Thompson FM, Arnold WD: Intraoperative radioactive localization of an osteoid-osteoma. case report. J Bone Joint Surg Am 198I, 63:826-827.

729. Colton CL, Hardy JG: Evaluation of a sterilizable radiation probe as an aid to the surgical treatment of osteoidosteoma. Technical note. I Bone Joint Surg Am 1983, 65: $1019-1022$

730. Harcke HT, Conway JJ, Tachdjian MO, Dias LS, Noble HB, MacEwen GD, Weiss S: Scintigraphic localization of bone lesions during surgery. Skeletal Radiol 1985, 13:2 II-216.

73I. Lacheretz M, Herbaux B, Hossein-Foucher C, Lecouffe P, Richir M: [Peroperative isotopic detection in the treatment of osteoid osteoma in children]. Chirurgie 1989, II5:413-416. [French]

732. Todd BD, Godfrey LW, Bodley RN: Intraoperative radioactive localization of an osteoid osteoma: a useful variation in technique. BrJ Radiol 1989, 62:187-189.

733. De Boeck H, Casteleyn PP, Bossuyt A, Jacobs A: Intraoperative radioactive localization of small bone tumours. Int Orthop 1992, 16:172-175.

734. Kirchner B, Hillmann A, Lottes G, Sciuk J, Bartenstein P, Winkelmann $\mathrm{W}$, Schober $\mathrm{O}$ : Intraoperative, probe-guided curettage of osteoid osteoma. Eur J Nucl Med 1993, 20:609-613.

735. Wioland $M$, Sergent-Alaoui A: Didactic review of 175 radionuclide-guided excisions of osteoid osteomas. Eur J Nucl Med 1996, 23:1003-1011.
736. D'Errico G, Rosa MA, Soluri A, Scafè R, Galli M, Chiarini S, Burgio N, Schiaratura A, Massa R, Scopinaro F: Radioguided biopsy of osteoid osteoma: usefulness of imaging probe. Tumori 2002, 88:S30-S32.

737. Tse WL, Hung LK, Law B, Ho PC: Enhanced localization of osteoid osteoma with radiolabeling and intraoperative gamma counter guidance: a case report. J Hand Surg [Am] 2003, 28:699-703.

738. Kettunen JS, Mäkelä EA, Kauppinen TA: Gamma probe localization in the surgical treatment of osteoid osteoma. Scand J Surg 2003, 92:224-226.

739. Matejka J, Záhlava J: [Vertebral osteoid osteoma - peroperative detection of its nidus using a surgical gamma probe]. Acta Chir Orthop Traumatol Cech 2003, 70:187-190. [Czech]

740. Etchebehere M, Etchebehere EC, Reganin LA, Amstalden EM, Cliquet A Jr, Camargo EE: Intraoperative localization of an osteoidosteoma using a gamma probe. Int Orthop 2004, 28:379-383.

74I. Van Royen BJ, Bayen JC, Pijpers R, Noske DP, Schakenraad D, Wuisman PI: Osteoid osteoma of the spine: a novel technique using combined computer-assisted and gamma probe-guided high-speed intralesional drill excision. Spine 2005, 30:369-373.

742. Pratali R, Zuiani G, Inada M, Hanasilo C, Reganin L, Etchebehere E, Etchebehere M: Open resection of osteoid osteoma guided by a gamma-probe. Int Orthop 2008 in press.

743. Harvey WC, Lancaster JL: Technical and clinical characteristics of a surgical biopsy probe. I Nucl Med I98I, 22:184-I86.

744. Krag DN, Ford PV, Patel M, Schneider PD, Goodnight JE: A simplified technique to resect abnormal bony radiolocalizations using a gamma counter. Surg Oncol I992, 1:37|-377.

745. Adams S, Hovy L, Baum RP, Adams M, Maul FD, Kahla-Witzsch H, Zichner L, Jonas D, Hor G: [Intraoperative localization of metastases with a hand-held gamma probe]. Nuklearmedizin 1995, 34:16I-164. [German]

746. Robinson LA: Radioisotope-guided surgical biopsy of suspected osseous metastases. Cancer Control 1997, 4:516-522.

747. Robinson LA, Preksto D, Muro-Cacho C, Hubbell DS: Intraoperative gamma probe-directed biopsy of asymptomatic suspected bone metastases. Ann Thorac Surg 1998, 65: 1426-I 432.

748. Albert GD, Alex JC, Krag DN, Weinberg DA: Gamma probe localization of cranial bone lesions. Ophthal Plast Reconstr Surg 1999, I5:470-472.

749. Fernandes DS, Aye RW, Garnett DJ, Denny J: Target-specific rib biopsy using the gamma probe. Am J Surg 2000, 179:389-390.

750. Uppot RN, Gheyi VK, Panasuk B, Sagar VV: Intraoperative gamma probe directed rib resection. Del Med J 2000, 72:385-388.

75I. van Mesdag T, Gommans GM, de Waard JW: [Radionuclideguided minimally invasive rib biopsy for metastases]. Ned Tiidschr Geneeskd 2002, I 46: I539-| 542. [Dutch]

752. Axelsson CK, Nielsen BP, Graff J: Radioisotope-guided surgical biopsy of costal metastases in breast cancer patients. Scand ] Surg 2002, $91: 333-335$

753. Thurman SA, Robinson LA, Ahmad N, Pow-Sang JM, Lockhart JL, Seigne J: Investigation of the safety and accuracy of intraoperative gamma probe directed biopsy of bone scan detected rib abnormalities in prostatic adenocarcinoma. J Urol 2003, 169:1341-1344.

754. Sodha S, Nagda S, Lackman RD, Donthineni R: Gamma probe assisted biopsy of suspected metastatic rib lesions. Clin Orthop Relat Res 2004, 422:186-189.

755. Andrade RS, Blondet JJ, Kast T, Jessurun J, Maddaus MA: Evaluation of isolated rib lesions with radionuclide-guided biopsy. Ann Thorac Surg 2008, 86: I | | |- | | |4.

756. Schattner A, Cohen A, Wolfson L, Melloul M: Unexplained systemic symptoms and Gallium-67-guided decisions. Am J Med Sci 2001, 321:198-200.

757. Burdine J, Joyce LD, Plunkett MB, Inampudi S, Kaye MG, Dunn DH: Feasibility and value of video-assisted thoracoscopic surgery wedge excision of small pulmonary nodules in patients with malignancy. Chest 2002, 122:1467-1470.

758. Stehlin JS Jr, Clark RL Jr, Dewey WC: Continuous monitoring of leakage during regional perfusion. Arch Surg I96I, 83:943-949.

759. Sardi A, Minton JP, Mojzisik C, Nieroda CA, Ferrara PJ, Hinkle GH, Thurston MO, Martin EW Jr: The use of a hand-held gamma detector improves the safety of isolated limb perfusion. J Surg Oncol 1989, 41:172-176. 
760. Manner M, Sinn H, Bubeck H, Kettelhack C, Schlag P: [Improved intraoperative leak control in cytostatic drug isolation perfusion of tumors of the extremities]. Langenbecks Arch Chir 1990, 375:208-213. [German]

76I. Sprenger HJ, Markwardt J, Schlag PM: [Quantitative radionuclide leakage control during isolated limb perfusion]. Nuklearmedizin 1994, 33:248-253. [German]

762. Barker WC, Andrich MP, Alexander HR, Fraker DL: Continuous intraoperative external monitoring of perfusate leak using iodine- I I I human serum albumin during isolated perfusion of the liver and limbs. Eur J Nucl Med 1995, 22: I 242-I 248.

763. Sandrock D, Horst F, Gatzemeier W, Ghorbani M, Rauschecker $H$, Munz DL, Emrich D: Leakage measurement during selective limb perfusion using a gamma probe. Eur J Nucl Med 1996, 23:534-538.

764. Daryanani D, Komdeur R, Ter Veen J, Nijhuis PH, Piers DA, Hoekstra $\mathrm{HJ}$ : Continuous leakage measurement during hyperthermic isolated limb perfusion. Ann Surg Oncol 200I, 8:566-572.

765. van Ginkel RJ, Limburg PC, Piers DA, Koops HS, Hoekstra HJ: Value of continuous leakage monitoring with radioactive iodineI 3 I-labeled human serum albumin during hyperthermic isolated limb perfusion with tumor necrosis factor-alpha and melphalan. Ann Surg Oncol 2002, 9:355-363.

766. Casara D, Rubello D, Pilati PL, Scalerta R, Foletto M, Rossi CR: A simplified procedure for continuous intraoperative external monitoring of systemic leakage during isolated limb perfusion. Tumori 2002, 88:S6I-S63.

767. Casara D, Rubello D, Pilati P, Scalerta R, Foletto M, Rossi CR: Optimized procedure of real-time systemic leakage monitoring during isolated limb perfusion using a hand held gamma probe and 99mTc-HSA. Nucl Med Commun 2004, 25:6I-66.

768. Hall NC, Povoski SP, Murrey DA Jr, Knopp MV, Martin EW Jr: Bringing advanced medical imaging into the operative arena could revolutionize the surgical care of cancer patients. Expert Rev Med Devices 2008, 5:663-667.

Publish with Bio Med Central and every scientist can read your work free of charge

"BioMed Central will be the most significant development for disseminating the results of biomedical research in our lifetime. "

Sir Paul Nurse, Cancer Research UK

Your research papers will be:

- available free of charge to the entire biomedical community

- peer reviewed and published immediately upon acceptance

- cited in PubMed and archived on PubMed Central

- yours - you keep the copyright
BioMedcentral 